\title{
Feasibility of enhanced user control and feedback in upper leg prostheses
}



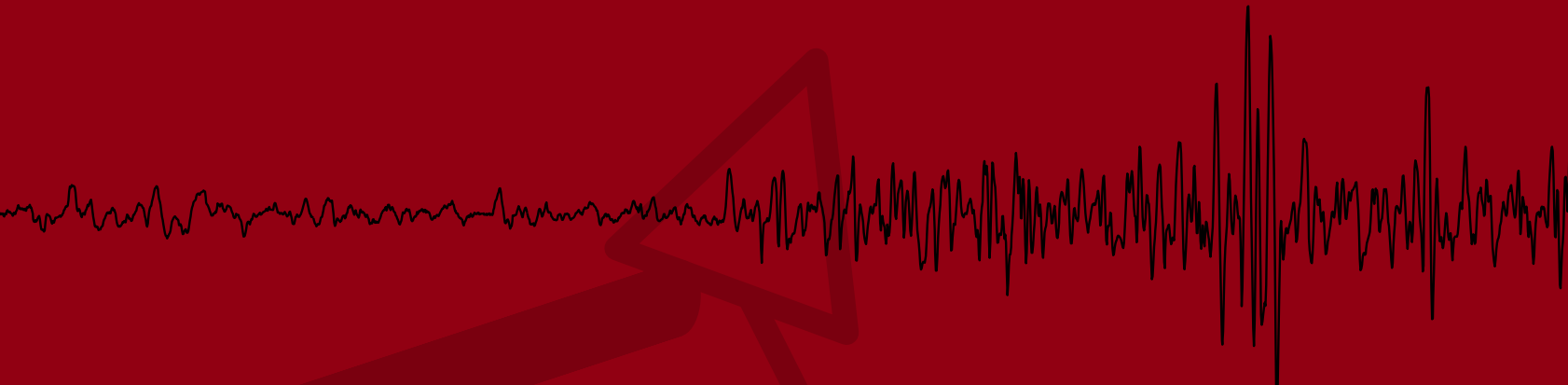

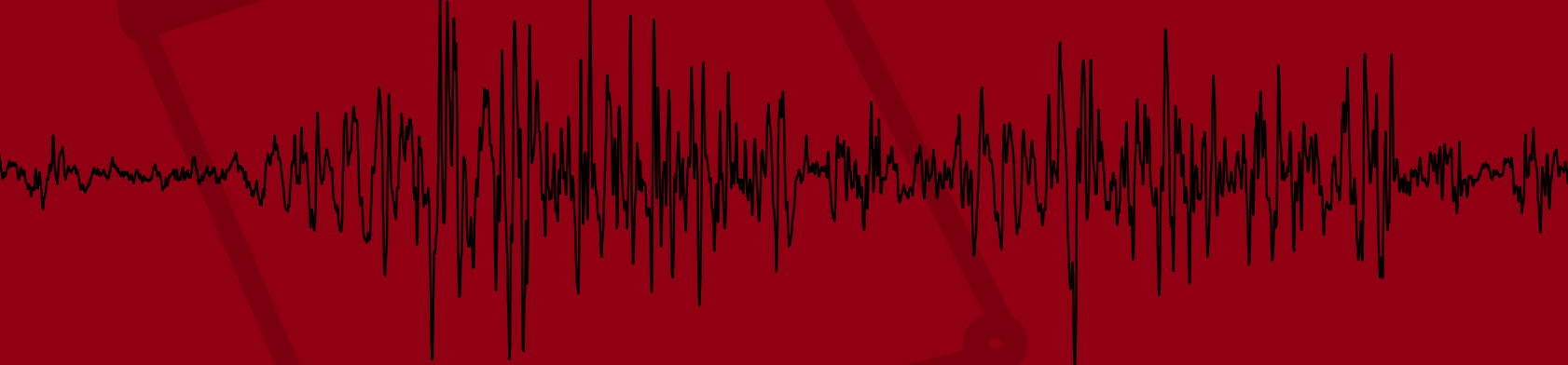


FEASIBILITY OF ENHANCED USER CONTROL AND FEEDBACK IN UPPER LEG PROSTHESES 


\section{Members of the graduation committee:}

Chairman and secretary:

Prof. dr. ir. A.J. Mouthaan University of Twente, Enschede, Nederland

Promotors:

Prof. dr. ir. P.H. Veltink University of Twente, Enschede, Nederland

Prof. dr. J.S. Rietman University of Twente \& Roessingh Research and Development, Enschede, Nederland

Members:

Prof. dr. ir. G.J. Verkerke University of Twente, Enschede, Nederland

Prof. dr. ir. H.J. Hermens University of Twente \& Roessingh Research and Development, Enschede, Nederland

Prof. dr. K. Postema University Medical Center, Groningen, Nederland Dr. ir. D.H. Plettenburg Technical University, Delft, Nederland

Funding for this research was provided by:

Dutch Technology Foundation STW, which is part of the Netherlands Organization for Scientific Research (NWO) and partly funded by the Ministry of Economic Affairs, Agriculture and Innovation, under grant no. 08003.

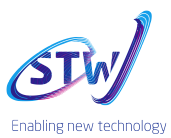

Publication of this thesis was generously supported by:

Biomedical Signals and Systems \& $\underline{\text { ös }}$.

\section{Life Without Limitations}

This work was carried out at:

Biomedical Signals and Systems group, MIRA Institute for Biomedical Technology and Technical Medicine, Faculty of Electrical Engineering, Mathematics and Computer Science (EEMCS), University of Twente, The Netherlands.

In close collaboration with:

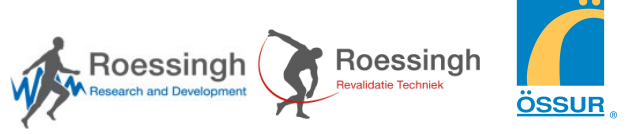

Author email: evacwentink@gmail.com

ISBN: 978-90-365-1195-7

DOI: $10.3990 / 1.9789036511957$

Copyright (C) 2013 by Eva C. Wentink 


\section{FEASIBILITY OF ENHANCED USER CONTROL AND FEEDBACK IN UPPER LEG PROSTHESES}

\section{PROEFSCHRIFT}

ter verkrijging van

de graad van doctor aan de Universiteit Twente,

op gezag van de rector magnificus,

prof. dr. H. Brinksma,

volgens besluit van het College voor Promoties

in het openbaar te verdedigen

op vrijdag 25 oktober 2013 om 16.45 uur

door

\section{Eva Christine Wentink}

geboren op 16 september 1982

te Enschede, Nederland 
Dit proefschrift is goedgekeurd door:

Prof. dr. ir. P.H. Veltink (Promotor)

Prof. dr. J.S. Rietman (Assistent promotor) 
Ga nooit weg zonder te groeten, ga nooit heen zonder een zoen. Wie het noodlot zal ontmoeten, kan het morgen niet meer doen.

Loop nooit weg zonder te praten, dat doet soms een hart zo pijn. Wat je 's morgens hebt verlaten, kan er 's avonds niet meer zijn.

Toon Hermans 



\section{Contents}

1 Introduction 1

1.1 Introduction . . . . . . . . . . . . . . . 1

1.2 Challenges in current prostheses . . . . . . . . 5

1.2.1 Control of prostheses . . . . . . . . . . . . 6

1.2.2 Feedback in prostheses . . . . . . . . . . . 9

1.3 Aim and outline of this thesis . . . . . . . . . . . 11

1.3.1 Thesis objectives . . . . . . . . . . . 11

1.3 .2 Part I . . . . . . . . . . . . . . . 12

1.3 .3 Part II . . . . . . . . . . . . . . . . . . 12

1.3 .4 Part III . . . . . . . . . . . . . . . . 13

I Reflexive control of a variable stiffness actuated knee

2 Reflexive control of a variable stiffness actuated knee - a $\begin{array}{ll}\text { model study } & 17\end{array}$

2.1 Introduction . . . . . . . . . . . . . . . . . . . . 18

2.2 Controllable spring concept . . . . . . . . . . . . 21

2.3 Continuous variable stiffness control . . . . . . . . . . 24

2.4 Methods . . . . . . . . . . . . . . . . . 26

2.4 .1 Simulations . . . . . . . . . . . . . 27

2.5 Results. . . . . . . . . . . . . . . . 31

2.5.1 Part 1 - Undisturbed stance phase . . . . . . . . 31

2.5.2 Part 2 - Disturbed stance phase . . . . . . . . . 31

2.5.3 Part 3 - Reflexive control of a disturbance . . . . . 32

2.6 Discussion . . . . . . . . . . . . . . . . 34 
2.6.1 Conceptual considerations . . . . . . . . . . . 35

2.6 .2 Modeling considerations . . . . . . . . . . . 37

2.7 Conclusions . . . . . . . . . . . . . . . . . 38

II Voluntary control of upper leg prostheses $\quad 39$

3 EMG of transfemoral amputees and controls during level walking 41

3.1 Introduction . . . . . . . . . . . . . . . . . 42

3.2 Methods . . . . . . . . . . . . . . . 43

3.2 .1 Participants . . . . . . . . . . . . . . 43

3.2 .2 Measurements . . . . . . . . . . . . . 45

3.2 .3 Procedures . . . . . . . . . . . . . . . 45

3.2 .4 Data analysis . . . . . . . . . . . . . . 46

3.3 Results . . . . . . . . . . . . . . . . . 47

3.3 .1 Kinematic data . . . . . . . . . . . . . . 47

3.3.2 EMG data. . . . . . . . . . . . . . . 51

3.4 Discussion . . . . . . . . . . . . . . . . . . 58

3.4.1 Kinematic and spatio-temporal data . . . . . . . 58

3.4 .2 EMG . . . . . . . . . . . . . . 59

3.5 Conclusion . . . . . . . . . . . . . . . . 62

4 EMG of transfemoral amputees and controls during slope and stair walking $\quad 63$

4.1 Introduction . . . . . . . . . . . . . . . . . . . 64

4.2 Methods . . . . . . . . . . . . . . . . . . 67

4.2 .1 Procedures . . . . . . . . . . . . . . . 67

4.2 .2 Data analysis . . . . . . . . . . . . . . . . . 68

4.3 Results. . . . . . . . . . . . . . . . 68

4.3.1 Spatio-temporal data . . . . . . . . . . . 68

4.3.2 EMG data. . . . . . . . . . . . . . . . . 72

4.4 Discussion . . . . . . . . . . . . . . . . . . . 81

4.4 .1 Slope . . . . . . . . . . . . . . 83

4.4 .2 Stair ascent . . . . . . . . . . . . . . 84

4.4.3 Stair descent . . . . . . . . . . . . . 86

4.4.4 Methodological considerations . . . . . . . . . 87

4.5 Conclusion . . . . . . . . . . . . . . 88 
5 Gait initiation detection in non-amputees $\quad 91$

5.1 Introduction . . . . . . . . . . . . . . . 92

5.2 Methods . . . . . . . . . . . . . . . 93

5.2 .1 Participants . . . . . . . . . . . . . 93

5.2 .2 Measurements . . . . . . . . . . . . 93

5.2 .3 Procedures . . . . . . . . . . . . . . . . . . . . 94

5.2 .4 Data analysis . . . . . . . . . . . . . . 95

5.3 Results. . . . . . . . . . . . . . . 97

5.3 .1 Ensemble Averages . . . . . . . . . . . . . 97

5.3 .2 Timings . . . . . . . . . . . . . . 97

5.4 Discussion . . . . . . . . . . . . . . . . . . . . 101

5.4.1 Mimicked prosthetic leg leading . . . . . . . . . . 101

5.4 .2 Mimicked intact leg leading . . . . . . . . . . . . . 101

5.4.3 Methodical considerations . . . . . . . . . . . 102

5.5 Conclusions . . . . . . . . . . . . . . . . 103

6 Gait initiation detection in transfemoral amputees 105

6.1 Introduction . . . . . . . . . . . . . 106

6.2 Methods . . . . . . . . . . . . . . . . . 107

6.2.1 Participants . . . . . . . . . . . . . 107

6.2.2 Measurements . . . . . . . . . . . . . . 107

6.2.3 Procedures . . . . . . . . . . . . . . 107

6.2.4 Data analysis . . . . . . . . . . . . . . 108

6.3 Results . . . . . . . . . . . . . . . . . . . 109

6.3 .1 Detection of IS and IM . . . . . . . . . . 109

6.3.2 Detection from EMG . . . . . . . . . . . 113

6.4 Discussion . . . . . . . . . . . . . . . . . . 117

6.4.1 Methodical considerations . . . . . . . . . 118

6.5 Conclusions . . . . . . . . . . . . . . . . . . 119

$\begin{array}{ll}\text { III Feedback in upper leg prostheses } & 121\end{array}$

7 Feedback in upper leg prostheses 123

7.1 Introduction . . . . . . . . . . . . . . . . . . 124

7.2 Vibrotactile feedback . . . . . . . . . . . . . . 127

7.2 .1 Introduction . . . . . . . . . . . . . 127

7.2 .2 Methods . . . . . . . . . . . . . 127

7.2 .3 Results . . . . . . . . . . . . . . . . . . 129

7.2 .4 Discussion . . . . . . . . . . . . . . . 132

7.3 Vibrotactile feedback inside the socket . . . . . . . . 134 
7.3.1 Introduction . . . . . . . . . . . . . . . 134

7.3 .2 Methods . . . . . . . . . . . . . . . 134

7.3 .3 Results . . . . . . . . . . . . . . 136

7.3.4 Discussion . . . . . . . . . . . . . . . 137

7.4 Continuous electrotactile feedback . . . . . . . . . 139

7.4.1 Introduction . . . . . . . . . . . . . . 139

7.4 .2 Method . . . . . . . . . . . . . . . 139

7.4.3 Results . . . . . . . . . . . . . . . . 142

7.4 .4 Discussion . . . . . . . . . . . . . . . . . . . 144

7.5 Error-based electrotactile feedback . . . . . . . . . 146

7.5.1 Introduction . . . . . . . . . . . . . . 146

7.5 .2 Methods . . . . . . . . . . . . . . . . . . 146

7.5.3 Results . . . . . . . . . . . . . . . . 149

7.5 .4 Discussion . . . . . . . . . . . . . . . 152

7.6 Conclusions . . . . . . . . . . . . . . . . . . . 154

8 General discussion $\quad \mathbf{1 5 5}$

8.1 Introduction . . . . . . . . . . . . . . 155

8.2 Reflexive variable stiffness controlled prosthetic knee . . 156

8.3 Voluntary control of a prosthetic knee . . . . . . . . . 157

8.4 Feedback in upper leg prostheses . . . . . . . . . . . 162

8.5 Conclusions . . . . . . . . . . . . . . . . 165

8.6 Future research . . . . . . . . . . . . . . . . 165

$\begin{array}{ll}\text { Bibliography } & 169\end{array}$

$\begin{array}{ll}\text { Summary } & 183\end{array}$

$\begin{array}{ll}\text { Samenvatting } & 185\end{array}$

$\begin{array}{ll}\text { Dankwoord } & 189\end{array}$

$\begin{array}{ll}\text { List of publications } & 193\end{array}$ 


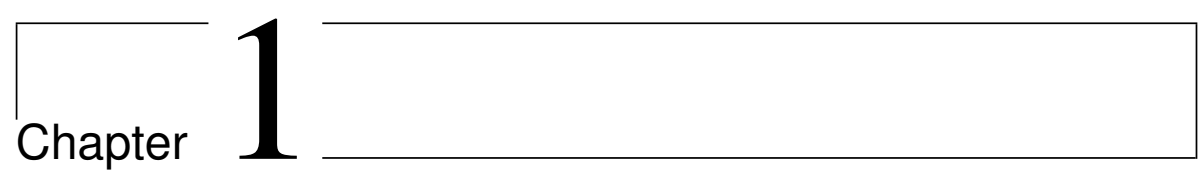

\section{Introduction}

\section{$1.1 \quad$ Introduction}

In the Netherlands every year around 700 people lose (part of) their lower limb $[1,2]$. Depending on where you are in the world the causes for amputations can be very different. In most developed countries 60$65 \%$ of all amputation is caused by diseases and only $30 \%$ by trauma. In other countries, Zimbabwe for is instance, trauma is with $95 \%$ the main reason for amputation. In the Netherlands the main causes are diabetes and vascular diseases and most patients with a transfemoral amputation are above the age of $60[1,2]$.

Two types of amputations require an upper leg prosthesis, the transfemoral amputation and the through knee amputation. The transfemoral amputation is performed on a more regular basis for it was believed to be best for the patient; to allow better prosthetic fitting. Nowadays the through knee amputation is also performed due to a more simple surgical procedure and the improvement of prostheses [2]. However, wound healing is often better after transfemoral amputations [2]. The through knee amputation has as main disadvantage that the prosthetic knee will be placed lower than the original and the contralateral knee. This is however mainly a cosmetic problem when seated. Some modern prosthetic knees can reduce this difference to a minimum.

The oldest known lower leg prosthesis is probably the Capua leg from 300BC, found in Italy in 1858. This was a large wooden base with a slight curve at the top to fit the residual limb (figure 1.1) and a bronze waist band. This was the predecessor of the peg-leg, which is 
no more than a stick replacing the leg. Ambroise Paré made the first prosthesis with a hinge at the knee around 1560, most of these knees would still lock during walking, but allowed the user to sit with a bend knee. In 1816, Potts made an upper leg prosthesis with a knee hinge that allowed flexion during walking. Elastic bands were used to force the knee into extension at the end of the swing phase. The design of the "socket" also changed, it was no longer just wood or copper, but leather was more comfortable. However only the rich could afford such a prosthesis, therefore the peg-leg was still used for an extensive period of time. Around WWI the first feet and knees were made and after WWII, the prosthesis as we know it today was formed. It was no longer one solid prosthesis, but the prosthesis became modular, with a socket, an upper leg where needed, a knee, a lower leg and a foot. Feet, knees and sockets became interchangeable to fit the users needs. Science and surgery currently complement each other to improve the prosthesis and its fitting. [2]

Current prostheses allow amputees to walk with a similar walking pattern as non-amputees do, but amputees still need to adapt to their prosthesis [3]. Mechanical knees give a stable knee in stance, but also allow flexion during the swing phase. They can be adjusted to the normal walking speed of the user. Modern microprocessor controlled knees automatically adjust the damping of the knee for different walking speeds or other activities, to better adapt to the amputees needs. Examples of these are the C-leg and Genium knees by Otto bock [4] and the Rheo knee by Ossur [5]. The only powered knee available on the market at the moment is the Power knee by Ossur [5]. This knee has all the advantages of the microprocessor controlled knee and it also assists the amputee in for instance walking upstairs, but it requires considerable powering by batteries attached to the prosthesis. [5]

Many amputees re-learn to walk on a prosthesis after the amputation. However, some amputees remain in a wheelchair for the rest of their life. The ability of amputees to walk after an amputation is subdivided into 5 categories or K-levels 0-4 [6].

K-Level 0 The patient does not have the ability or potential to ambulate or transfer safely with or without assistance and a prosthesis does not enhance his/her quality of life or mobility.

K-Level 1 The patient has the ability or potential to use a prosthesis for transfers or ambulation on level surfaces at fixed cadence. 
K-Level 2 The patient has the ability or potential for ambulation with the ability to traverse low level environmental barriers such as curbs, stairs, or uneven surfaces.

K-Level 3 The patient has the ability or potential for ambulation with variable cadence and the ability to traverse most environmental barriers.

K-Level 4 The patient has the ability or potential for prosthetic ambulation that exceeds basic ambulation skills, exhibiting high impact, stress, or energy levels.

These K-levels are an indication to which prosthetic components, e.g. knee and foot, are best suited for the amputee. Nowadays there are many prosthetic components to choose from, the time of the peg-leg is long gone. 


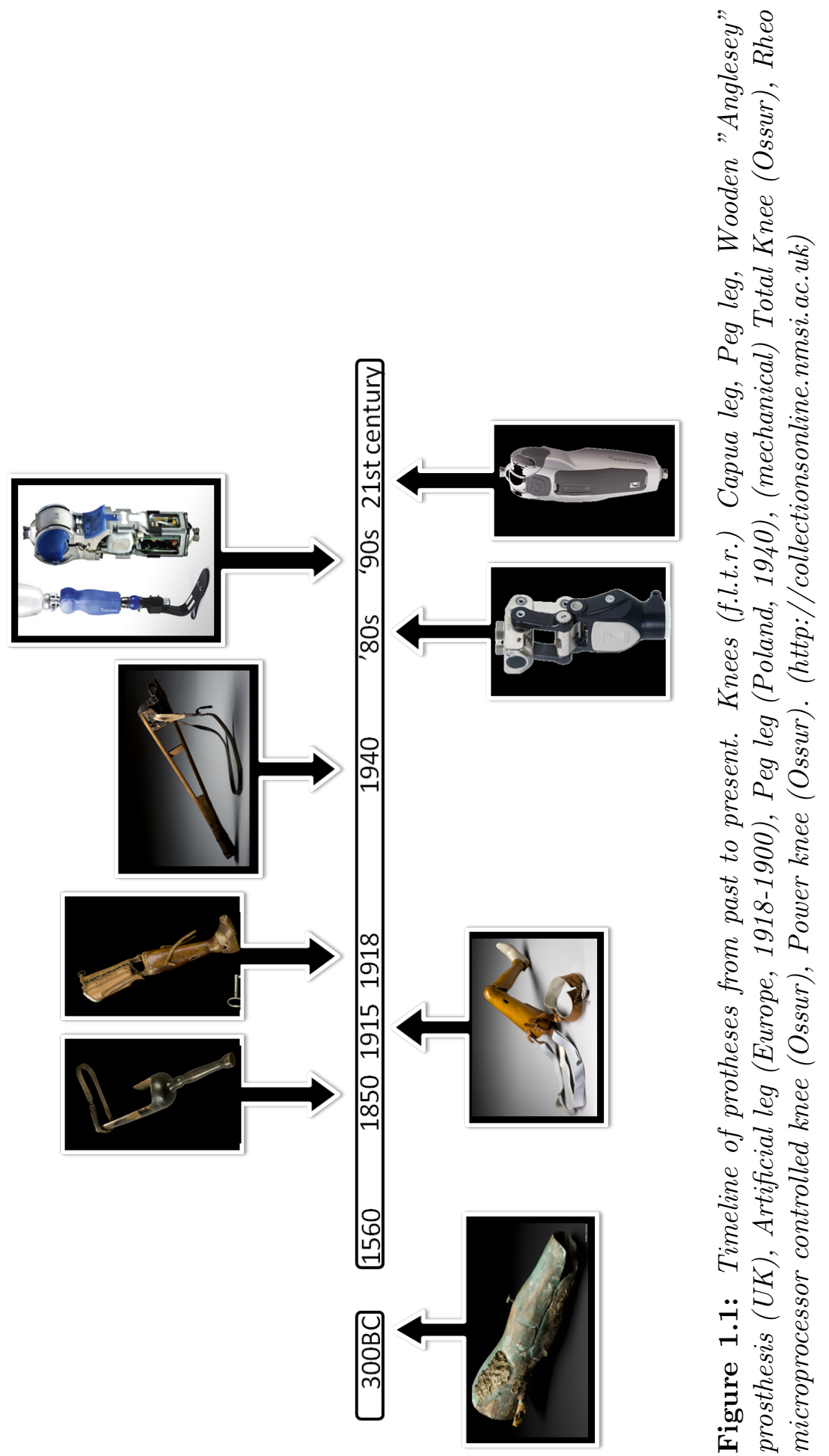




\subsection{Challenges in current prostheses}

There are however still some important challenges. Many amputees are insecure about their prosthesis, are afraid of falling or actually fall. All amputees require more energy to walk than a non-amputee and many even decide not to wear a prosthesis at all. [7-9] The insecurity may be caused by the lack of feedback about the state of the prosthesis and limited control of the prosthesis.

During normal walking, or other daily activities, a non-amputee can control the movements of the leg. The leg is controlled almost automatically for different activities and proprioception gives the subject feedback about the position of the leg in space, but also about for instance muscle tension. [10] In non-amputees a sudden knee-unlock would immediately result in a reflex from the proprioceptors to the central nervous system (CNS). This reflex serves as an input for the quadriceps and the hamstrings to react to the unforseen situation [10]. At the same time the other limb will also be activated by the cross-reflex, ensuring stability at all times. All this occurs in about 50-70 ms [10-12].

Gait in non-amputees is relatively energy efficient. During walking energy is absorbed and generated at the ankle and knee. In the stance phase the knee absorbs energy during flexion which is about the same amount of energy that is needed for the extension of the knee $[13,14]$. The ankle also absorbs and subsequently dissipates energy during the stance phase, but generates power during the push-off phase. During the push-off and swing phase the knee absorbs (and dissipates) energy again. The total amount of energy absorbed at the ankle and the knee during one gait cycle $(0.33 \mathrm{~J} / \mathrm{kg})$ is almost equal to the amount of energy generation at the knee and ankle $(0.35 \mathrm{~J} / \mathrm{kg})$. [13,14] Walking could therefore be almost fully energy efficient, if muscles were able to re-use the absorbed energy, rather than dissipating it.

Metabolic energy consumption during gait in TFA is considerably higher (up to 60\%) compared to non-amputee walking [9]. No energy is generated at the prosthetic knee and ankle. All the energy needed for walking with an upper leg prosthesis must be generated at the hip on the prosthetic side, or from hip, knee and ankle at the intact side. Current prostheses generally work with dampers, therefore energy is absorbed in the prosthetic knee, however this energy is not stored but dissipated. Springs can store energy during compression or lengthening, and return that energy when the spring is released. If springs can be 
made controllable such that the stiffness, time and amount of energy absorbtion/release can be controlled, they may become very useful for application in prosthetic knees [14].

In transfemoral amputees (TFA) no proprioception and motor control is available from the knee and below to react to an unforseen situation. Although microprocessor-controlled knees can react to sudden perturbations, by increasing the damping of the knee, proprioception and voluntary control are still not implemented. [4,5] Besides the haptic interface between residual limb and socket, these knees do not allow for any interaction to and from the user. In case of an unforseen situation the amputee generally only becomes aware of it after the prosthetic knee has reacted or when it is already too late to react. Most users therefore still rely on visual feedback or auditive cues to determine for instance knee-lock at the end of the swing phase. However these techniques require a lot of mental effort from the TFA. [15-18] The question is if the addition of artificial proprioception to the prosthesis, by means of sensory feedback about the state of the prosthesis, will give the the TFA more trust and awareness.

Intuitive voluntary control of the prosthetic knee may give the amputee more awareness and confidence. $[7,8]$ In current prostheses the amputee can only actively control the socket, using the haptic interface, and thereby the lower leg. Allowing the user, rather than the knee itself, to voluntarily control the damping of the knee or even knee flexion or extension may provide more trust and a better awareness of what the prosthesis will do. If voluntary control is intuitive and complemented by sensory feedback, the amputees will become fully aware of the state of the prosthesis and can control its motion. Ideally the user becomes part of a reflexive loop, similar to the proprioception and motor control loop a normal human leg has. However, at the moment it is unclear if this is feasible. The following paragraphs will give a short overview of previous attempts to voluntary control (upper leg) prostheses and provide feedback in (upper leg) prostheses.

\subsubsection{Control of prostheses}

Inertial sensors and force sensors inside modern microprocessor controlled knees measure kinematics and kinetics. From this data it is determined in which part of the gait cycle the TFA is and subsequently the 
damping of the knee is controlled. This however only allows the TFA to control the prosthesis by moving the residual limb, which subsequently has its effect on the knee rather than controlling the knee itself by using the muscles.

To control a prosthesis according to human motion we first need to analyze how the amputee moves. Human movements are generally analyzed using kinetics, kinematics and electromyography (EMG) [19-22]. Kinetics describes the forces that cause a movement, kinematics describe the actual human motion without considering the forces. EMG reflects the activation of skeletal muscles by the central nervous system. An active muscle produces more electrical activity than a relaxed muscle. Kinetics and kinematics of transfemoral prosthetic walking has been investigated in previous studies [23-26]. EMG on the other hand has been widely studied in non-amputee walking [19-22], but only few studies determined the EMG patterns during amputee gait [27].

Many new prosthetic designs are tested on non-amputees using prosthetic simulators. However, there is little information available on the differences in kinematics and EMG between amputees and non-amputees for different activities, therefore results from new designs tested on nonamputees may not directly be applicable in amputees. More information on the kinematic and EMG patterns of transfemoral amputees during daily life activities may provide more insight in how they adapt to their prosthesis, but also on where, when and how an upper leg prosthesis can be voluntary controlled.

Voluntary control of a prosthetic upper limb is not uncommon. Several control methods are available for upper extremity prostheses. The most common one used to be body powered, with wires and cables connected to the shoulder and the other arm. [28,29] Nowadays EMG is widely used. Muscle activity of forearm muscles is used to control hand opening and closing, and rotation of the wrist. [4,29] TouchBionics designed the i-limb, which has multiple hand and finger motions controlled by EMG and has varying grip strengths by the hand itself [30]. Targeted reinnervation of a muscle to control an upper extremity prosthesis has been performed by Kuiken et al. [31]. They reinnervated the pectoralis muscle with nerves previously innervating the amputated parts of the arm. EMG from the pectoralis muscle was subsequently used to successfully control an upper extremity prosthesis. Targeted reinnervation is a highly invasive method for providing control and mostly only suitable for very high amputations. For the lower extremities we aimed at using 
non-invasive methods for use of prosthetic control and therefore invasive methods will not be further discussed in this thesis.

For the lower extremity however few studies are known that have looked into voluntary control of a prosthesis. Previous EMG measurements, although mostly performed in healthy subjects, showed that in combination with pattern recognition techniques, it was possible to detect knee flexion or extension [32], perform terrain identification [33] or perform intent recognition for prosthetic control [34-37]. Measuring EMG inside the socket of a TFA has proven to be possible [27,38].

Aeyels et al. [39] designed an upper leg prosthesis which used EMG to complement pressure sensors and knee angle measurements to determine gait phases and subsequently control the braking system inside the prosthetic knee. The prosthesis could only control a finite number states of the knee.

In a study by Hargrove et al. [40] four transfemoral amputees were asked, while seated, to (virtually) perform several motions of the knee and ankle. EMG patterns recorded during these motions were used to classify the different motions per subject. Subsequently they were asked to replicate motions in a virtual environment, using real-time EMG. Motions were correctly detected in amputees in 70 to $97 \%$ of the trials and were performed in 1.5-5 seconds, but all whilst seated. [40]

Hoover et al [41] designed a prosthetic knee that could adjust the impedance of the knee by EMG of the vastus lateralis and the biceps femoris. They showed in a case study that stair ascent is possible using this type of EMG controlled powered prosthetic knee [42]. The nominal knee impedance was adjusted using information about ground contact of the foot. Extensive training was provided to the subject, first without socket to reduce the amount of co-contraction and later in training sessions [42]. However, amputees use also co-contraction to improve socket fitting, which may make it difficult to implement this type of control $[24,26,27,41]$. Zhang et al. [36] showed in one amputee detection of the beginning of the swing phase from stance to walking using EMG, up to $152 \mathrm{~ms}$ before the event. They used a custom made socket and did not mention which limb was leading.

Although the above described studies have demonstrated possibilities for implementation of voluntary control in upper leg prostheses, so far they were also rather slow, required extensive training or were only tested on non-amputees or under non-weight bearing conditions. Despite the above mentioned efforts, no EMG controlled upper leg prostheses are commercially available yet and more research is needed. 


\subsubsection{Feedback in prostheses}

Only few attempts have been made to integrate feedback into an upper leg prosthesis. Some of the issues in lower extremity feedback are timing and interpretability of the feedback. Timing is critical; if feedback is given too late or takes too long to be interpreted, it may lead to a fall or it is simply not useful. Making the amputee part of a reflexive feedback and control loop between residual limb and prosthesis will allow the user to intervene with any unexpected events. If well implemented, it will also trigger the cross-reflex, involving the contralateral limb for increasing the overall stability. Reflexive control allows the upper leg prosthesis to become second nature to the user [43].

Many different methods, invasive and non-invasive, are available to provide feedback. Myodesis, reattaching muscle to bone or myoplasty, reattaching muscle to muscle are used during amputation surgery, but mainly to ensure proper residual limb reconstruction and control rather than for the use of feedback [44]. Cineplasty, reattaching muscles to a prosthesis would be an ideal way of providing feedback and control, but has so far only been performed for upper extremities and is highly invasive [44].

Targeted reinnervation has also proven to be successful as feedback. Although also highly invasive, even sensory information has been reported to return. [31,45] Nerve stimulation is also a form of sensory feedback. By placing a wire electrode in the nerve or a cuff around the nerve information can be provided to a subject [46,47]. Clippinger et al. $[48,49]$ implanted a sciatic nerve stimulator inside the residual limb. The knee bending moment was presented to the subject by the stimulator using information from strain gages and a pressure activated piezo-electric crystal in the prosthesis. They reported that sensory stimulation relieved phantom pain of patients and increased their walking confidence due to a better awareness of the center of gravity. Although they claimed that their method was successful, no follow up was found, nor did the method became commercially available. However, invasive methods of applying feedback will not be further discussed in this thesis.

In current prostheses haptic feedback is the only feedback a TFA receives which may be artificially enhanced to present the user with more specific feedback. However, the relation between socket pressure and 
the knee angle is unknown. To provide adequate feedback to the prosthetic user via the socket-residual limb interface, more insight is needed in the socket pressure. [38] Fan et al. [50] designed a feedback method for the upper leg using air cushions. Information on pressure, sensed under the foot, was translated to four balloons in a cuff around the upper leg. However, seated healthy subjects took, up to 1-2 seconds to interpret the feedback. Koritnik et al. [51] gave haptic feedback with an actuated gait orthosis to healthy subjects in lower extremity training. They found that the task performance of stepping-in-place using haptic feedback improved, and was in general better than when using visual feedback from a virtual reality coach.

Real-time virtual reality full-body representation as visual feedback has a positive effect on the walking pattern and rehabilitation of amputees [52]. Auditory feedback has also been used to provide feedback when gait is asymmetric or weight-bearing too low. It appeared to be effective in training lower limb amputees during rehabilitation. [53,54]

Tactile stimulation of the skin is a different way of providing feedback. Prior studies in upper extremity prostheses have shown that electrotactile and vibrotactile feedback might be of additional value. For upper extremity prostheses this form of feedback was also used in for instance, the "Boston Arm" by Mann et al [55] and a hand prosthesis by Pylatiuk et al. [56]. Witteveen et al. [57] showed that subjects receiving feedback on hand opening and touch without visual feedback, performed better than without any form of feedback. Force and slip feedback were also successfully fed back to healthy subjects and amputees using vibrotactile stimulation [58]. However, timings of the performed tasks were not considered important and correct detection rates were between 30 and $80 \%[57,58]$. Because timing of feedback in lower extremity prostheses is critical and errors might have serious consequences (e.g. falling) further research is required to determine whether vibrotactile or electrotactile feedback can be applied effectively. The above results have shown that feedback at the forearm is of additional value if no visual feedback is applied, and therefore if the results can be improved it may also become useful for the lower extremity.

No studies were found on vibrotactile feedback at the upper leg. Electrical stimulation of the skin has previously been used for feedback on the lower extremity. Vos et al. [59] used an array of electrodes to provide feedback at the upper leg. Detection of disturbances was possible using an array of electrodes. They found that disturbed walking patterns 
projected on the upper leg were correctly detected in about $95 \%$ of the trials, during continuous stimulation.

\subsection{Aim and outline of this thesis}

The research described in this thesis is part of the STW project Reflexleg, which is performed by the Biomedical Signals and Systems, the Biomechanical Engineering and the Control Engineering groups of the University of Twente in close collaboration with Roessingh Research and Development. The Reflex-leg project aimed at designing a prosthesis that could be controlled reflexively and energy efficient without any (invasive) interventions to the amputee.

One part of the project aimed at making the prosthesis more efficient. By using controllable springs rather than dampers to control the knee, energy can be stored during walking and returned to the user during for instance push-off.

The other part of the project aimed at integrating the user into the control and feedback loop. If the user can control the prosthesis and at the same time receives feedback from it, than the use of the prothesis may become more natural. Ideally the user becomes part of a reflex-loop: the prosthesis gives feedback to the user, the user reacts reflexively and intuitively controls the prosthesis.

The findings of the second part of the Reflex-leg project will be described in this thesis. Figure 1.2 shows a schematic overview of the proposed concept (left and right) and the current control inside the prosthetic knee (right).

\subsubsection{Thesis objectives}

This thesis has three main objectives related to the proposed reflexive control and feedback loop, each described in a separate part of the thesis.

I Assess the feasibility of the prosthetic user becoming part of the feedback and control loop of a variable stiffness actuated prosthetic knee.

II Increase the insight in kinematics and residual limb EMG of transfemoral amputees and the usability of these data for voluntary control of an upper leg prosthesis. 


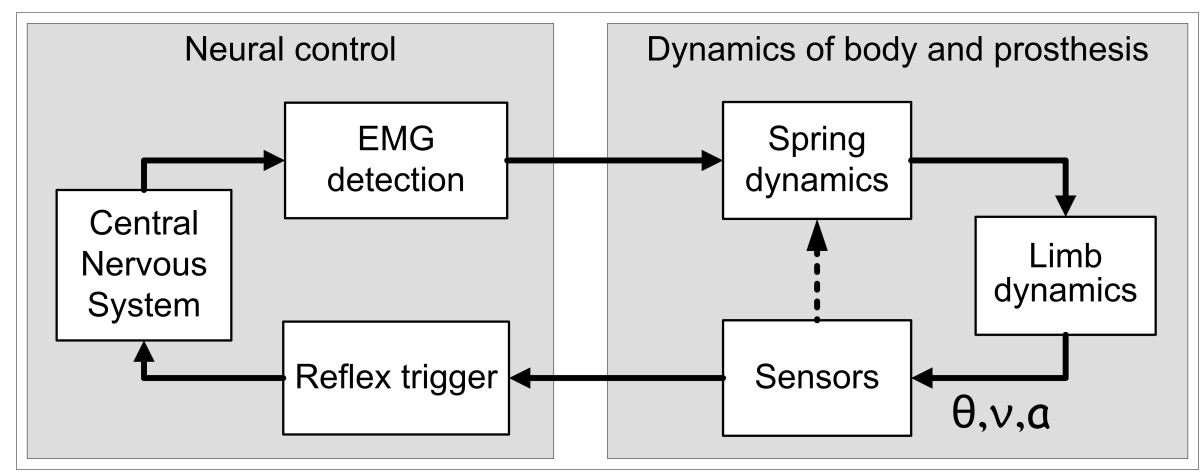

Figure 1.2: The control scheme proposed for the Reflex-leg project. On the right the control scheme inside the knee, on the left the proposed extension of the control scheme with the user inside the loop.

III Develop and evaluate a method of providing interpretable feedback from the prosthesis to the transfemoral amputee, in which the amputee must benefit from the feedback when walking with an upper leg prosthesis.

\subsubsection{Part I}

In the first part (Chapter 2) we investigated if the user can be part of the reflex-loop, combined with the energy efficient approach of using controllable springs. In this part a new concept of energy efficient control of a prosthetic knee is introduced, using variable stiffness actuation. We first analyzed if this concept can be used to reject a small disturbance. Together with this a feedback loop as shown in figure 1.2 is introduced to the control scheme to predict if the closed-loop system can be fast enough with the user inside the loop.

\subsubsection{Part II}

In the second part we first determined the kinematics and EMG activity patterns during daily activities of amputees and compared them to those of control subjects. This gained more information on how a prosthesis is currently controlled and in which way we can add control to a prosthesis. Although research had already shown that EMG can be measured inside the socket of an amputee, most researchers still use experimental sockets. We measured EMG in the socket of amputees without modifications and determined if the general walking patterns 
are still measurable. This was done for level walking (Chapter 3) and for slope and stair walking (Chapter 4). Thereafter we looked at the possibilities of predicting user activities from inertial sensor data and EMG. We first determined if gait initiation can be predicted from nonamputees (Chapter 5). Subsequently we determined if this could also be performed on data from transfemoral amputees (Chapter 6).

\subsubsection{Part III}

The third part describes and evaluates several methods for providing feedback in upper leg prostheses. We already excluded invasive methods of providing feedback and therefore we examined two tactile feedback modalities, electrotactile feedback and vibrotactile feedback, and auditory feedback. Only few studies have looked at these two modalities for feedback at the upper leg. Visual feedback is always available, but attention for the prosthesis and the surrounding is needed for it to be effective. Auditory feedback is a very strong feedback method, but may be disturbed in busy surroundings. Haptic feedback is always present in current upper leg prostheses through to the socket-residual limb interface $[38,50]$. Artificial feedback should be of additional value to the user, in addition to the visual and haptic feedback. There is limited information available about which frequency and on what location tactile stimulation at the upper leg is best perceived. Continuous feedback in an array or discrete feedback at a specific location and timing had not been investigated for either of the modalities, nor had feedback inside the socket. Chapter 7 describes the experimental results regarding several of these issues for vibrotactile and electrotactile feedback.

Chapter 8 involves the main results and the general discussion of this thesis. This chapter also includes a general conclusion and the implications of the findings for future research. 
Chapter 1 


\section{Part I}

\section{Reflexive control of a variable stiffness actuated knee}



Chapter 2

\section{Reflexive control of a variable stiffness actuated knee - a model study ${ }^{1}$}

Energy efficient variable stiffness control (VSA) can reduce the energy consumption yet effectively modulate the dynamic behavior and use stored energy during flexion to assist in subsequent extension. Adding reflexive user control and feedback may also increase awareness and trust in a prosthesis.

A principle design of energy efficient VSA in a prosthetic knee is proposed and analyzed for the specific case of rejection of a disturbed stance phase. The concept is based on the principle that the output stiffness of a spring can be changed without changing the energy stored in the elastic elements of the spring. The usability of this concept to control a prosthetic knee is evaluated using a model.

Part of the stance phase of the human leg was modeled by a double pendulum. Specifically the rejection of a common disturbance, an unlocked knee at heel strike, was evaluated. The ranges of spring stiffnesses were determined such that the angular characteristics of a normal stance phase were preserved, but disturbances could be rejected. In addition reflexive control was modeled by increasing the time delay in the system. The simulations predicted that energy efficient VSA can be useful for the control of prosthetic knees, but that reflexive control is too slow.

\footnotetext{
${ }^{1}$ Based on manuscript "Feasibility of energy efficient variable stiffness actuation to control a prosthetic knee - a modeling study", Published in: Medical Engineering and Physics 35(6):838-845 2013
} 


\subsection{Introduction}

Design of micro-processor controlled upper leg prostheses have lead to improved efficiency and walking comfort, but (metabolic) energy consumption during walking in transfemoral amputees (TFA) is still higher than in normal walking $[18,60]$. In robotics, studies on energy efficient actuation are not uncommon [61-63], but in prosthetics this is not the case. In this study we address the usability of energy efficient variable stiffness actuation for control of a prosthetic knee in the rejection of the consequence of an unlocked knee at heel strike. In addition we determined the possibilities of providing feedback and reflexive control to the user.

Most TFA wear mechanically passive prosthetic knees, such as the Mauch SNS and the Total knee by Ossur or the 3R55 by Otto Bock, suitable for walking at a specific speed, non-adaptive and without stance phase control [64]. Many of these knees have no safety mechanism, if the TFA lands on an unlocked knee a fall is inevitable. Mechanical knees do not need an external power source, such as the micro-processor controlled (MPC) or powered knees, they are fully passive.

MPC knees, like the Rheo-knee by Ossur and the C-leg by Otto Bock, require a little less metabolic energy of the TFA, provide more walking comfort and increase the quality of life with respect to conventional knees [60]. Different walking speeds and walking down the stairs (step after step) is made possible. Damping mechanisms in these knees prevent a collapse if the patient lands on a flexed knee, but do not help to extend the knee. Kuo and Donelan described dynamic walking and found that it may cost substantial amount of energy to support body weight on a bend leg, especially if it has to be extended again [65]. Dampers dissipate energy, whereas the original muscles and tendons act more like springs, storing elastic strain energy and restoring it where possible $[19,66]$. However, by making these dampers highly adaptable they can be tuned to resemble a natural motion more closely, making walking energy efficient even though the actuator itself is not energy efficient $[60,67]$.

The Power knee by Ossur is an externally powered intelligent knee, which can actively support the user during for instance walking, stair walking and slope walking. However, none of the previously mentioned knees are capable of storing and restoring energy in normal walking dur- 
ing the stance and swing phase $[14,19]$. The use of springs in prostheses could reduce the energy consumption. Springs are already used in current prosthesis, a few use springs for active knee extension during the swing phase, such as the Stabilized knee (Ohio Willow Wood). The XT9 (Symbiotechs, USA) gives additional support in extreme sports but is unsuitable for walking. Both Au et al. [68,69] and Zhu et al. [70] designed an ankle which stores energy during stance in a spring and releases this at push-off.

Sugar et al. [71] build "SPARKy", a prosthetic Spring Ankle with Regenerative Kinetics. This is a motorized robotic tendon and added springs parallel and in series to alter the energy, power and load requirements [71]. Unal et al. [14] build a prototype of a passive upper leg prosthesis, which is able to store energy absorbed during the stance and swing phase at knee and ankle and release it all at the ankle during pushoff. This prototype however also lacks control of the spring stiffnesses for different activities or walking speeds and for rejecting unexpected disturbances. An adjustable spring combines an energy efficient actuator with adaptability, which would allow an even more energy efficient system.

The use of adjustable springs in robotics is not new. Variable stiffness actuation (VSA) is the current solution in robotics to mimic muscles and tendons that still have superior characteristics with respect to power and adjustability $[63,66,72]$. Several studies have investigated VSA to control a joint by changing the stiffness of the joint $[62,63,72]$. Shen and Goldfarb designed an actuator which was able to simultaneously change the actuator output force and stiffness [73]. Filippini et al. [63] investigated several ways to implement agonist-antagonist actuation. Vanderborght et al. [62] designed the MACEPPA, the mechanically adjustable compliance and controllable equilibrium position actuator. In the MACEPPA the joint stiffness can be controlled by a lever arm or a heartshaped disk and pretensioning of the spring [62,72]. Braun and Goldfarb modeled a fully actuated biped robot, with a low-gain spring and damper system. This gave the biped natural walking dynamics to walk stable without prescribing the kinematic trajectories or constraints [74]. Visser at al. [61] designed a VSA setup which allows the apparent output stiffness of the actuator to be changed without changing the potential energy stored in the elastic elements. Another actuator concept, the V2E2, was proposed by Stramigioli et al. [75], which describes the combination of an Infinite Variable Transmission and an elastic element, to 
reduce energy loss during negative work done by the actuator.

Besides increasing energy efficiency, enhancing user control and feedback of the upper leg prostheses may also be beneficial for the prosthetic user. It can improve the trust and awareness in the prosthesis and possibly reduce the number of falls $[7,8]$. Current microprocessor controlled knees can reject small disturbances $[4,5]$. There is however no feedback to the user about these disturbance rejections. The user has only limited haptic feedback via the mechanical interaction between the residual limb and the prosthesis. Ideally the user becomes part of the feedback and control loop reflexively. To implement reflexive control in an upper leg prosthesis, an additional control loop inside the prosthetic knee needs to be included. The prosthesis should transfer detected disturbances to the user by eliciting reflexes, which may be modulated by the user in order to influence the response to the disturbance. The reaction by the user to the reflex, controls the muscles in the residual limb. This reflexive reaction in the muscles can be measured by electromyography and interpreted into a control signal to the prosthesis. The prosthesis subsequently reacts to the disturbance. Part of the objective of this modeling study is to assess the feasibility of such reflexive user control of a prosthesis. The main question to be answered is whether the time delay in this reflexive user control loop can be sufficiently small to be effective.

Characterization of parts of this loop have been previously described in literature: disturbances in transfemoral prosthetic walking have successfully been detected within 50 - $70 \mathrm{~ms}$ [76]. Triggering of artificial reflexes at the skin has previously been performed under the foot [77]. First reactions in EMG onset were detected between 40 and $50 \mathrm{~ms}$ in the lower leg muscles [77]. To detect EMG onset and determine if a muscle is active several onset detection algorithms are available. All these algorithms need time to determine the onset and also to determine the subsequent control action. Time delays have been reported between 10 and $250 \mathrm{~ms}$ for accurate EMG onset detections [78, 79]. The knee itself will also have a time delay, due to the sampling frequency. Current knees have sampling rates around $100-1000 \mathrm{~Hz}[4,5]$. To measure EMG a sampling frequency of $400 \mathrm{~Hz}$ is advised [80]. Therefore the estimated time delay in the knee lies around $5 \mathrm{~ms}$.

This implies that a minimal time delay of $105 \mathrm{~ms}$ needs to be added to the control loop, to implement reflexive control. In the current modeling study, we will analyze whether this delay can be expected to result in effective reflexive user control. 
In this study we investigated the possibilities to use energy efficient VSA to actuate the knee joint of a prosthesis during undisturbed gait and in reaction to a disturbed knee extension at the beginning of the stance phase. A controllable spring can allow control of the knee angle and energy storage during knee flexion and re-use of this energy to restore knee extension [81]. This is in contrast to a damper which only dissipates the energy to control the knee angle.

First we will introduce a concept of a controllable spring in a prosthetic knee. Secondly the feasibility of the general concept to reflexively control a (disturbed) knee is evaluated in a modeling study. For this purpose we modeled a prosthetic leg and simulated a normal and disturbed stance phase of gait, from heel strike until push-off whereby the knee was controlled using energy efficient VSA. The reflex loop was modeled using a time delay.

\subsection{Controllable spring concept}

The concept of a controllable spring is based on the principle that the effective (rotational) output stiffness can be changed without changing the energy stored in the internal elastic elements. Visser et al. [61] demonstrated that the output stiffness of a linear spring can be changed by changing the effective lever arm without adding energy. A similar setup can be used at a (knee) joint.

Figure 2.1(a) shows an example of how energy efficient VSA as proposed by Visser et al. [61] can be used for knee stiffness control. The lower end of linear spring is attached to the ankle and the top end is attached to the knee with a lever arm. The lever arm has a length $q_{1}$ and is placed at an angle $\left(q_{2}\right)$ with the upper leg. The energy in the spring is given by $E_{k}=\frac{1}{2} k\left(x-x_{0}\right)^{2}$, where $x_{0}$ it the unstretched length of the spring. The effective rotational output stiffness $\left(k_{R E}\right)$ is defined as the ratio of the infinitesimal change of the actuator output moment $M_{\text {spring }}$ as a result of an infinitesimal change in the joint angle $\theta$, on which the spring acts.

$$
k_{R E}=\frac{d M_{\text {spring }}(\theta)}{d \theta}
$$

At a certain (fixed) $q_{2}$ the force in the spring $\left(F_{s}\right)$ will be perpendicular to the lever arm at a specific knee angle $\left(\theta_{\text {thres }}\right)$. In this specific case changing $q_{1}$ will only change $k_{R E}$, but it will not not change the 
length of the spring $(\mathrm{x})$ and therefore it will also not change the spring energy $\left(E_{k}\right)$. The following holds only for this specific case where $F_{s}$ is perpendicular to the lever arm.

$M_{\text {Spring }}$ can be described as $F_{s} \cdot q_{1}=k\left(x-x_{0}\right) \cdot q_{1}$. The change in spring length is given by $d x=q_{1} \cdot d \theta$. The relation between $q_{1}$ and $K_{R E}$ follows from:

$$
\begin{gathered}
k_{R E}=\frac{d M_{\text {spring }}(x)}{d x} \frac{d x}{d \theta} \\
k_{R E}=\frac{d\left(q_{1} k\left(x-x_{0}\right)\right)}{d x} \frac{d\left(q_{1} \theta\right)}{d \theta}
\end{gathered}
$$

Resulting in:

$$
k_{R E}=q_{1}^{2} k
$$

Equation 2.4 shows that changing $q_{1}$ changes $k_{R E}$ quadratically. If $q_{1}$ is zero the spring will not exert a moment around the knee. $k_{R E}$ can therefore be changed in the range $\left[0, k_{R E, \max }\right]$. Where $k_{R E, \max }$ should be chosen such that it is enough to overcome a disturbance within a certain range. $q_{1}$ also has a maximal length $q_{1, \max }$ due to size restrictions of a prosthetic knee. The required spring stiffness $k$ can now be calculated using 2.5:

$$
k=\frac{k_{R E, \max }}{q_{1, \max }^{2}}
$$

A rough setup of how this can be achieved mechanically is shown in figure 2.1(b). The lever arm and the upper leg (socket) can be mechanically linked (at an angle $q_{2}$ ). The attachment point (P) of spring and lever arm should be movable, but blocked by a lock. The lock can be linked to the lower leg, which at the desired knee angle will dislocate the lock and allow $\mathrm{P}$ to move to the end of the lever arm. Switching (too) early, $\theta<\theta_{\text {thres }}$, will shorten the spring. This may however be beneficial to ensure that $\mathrm{P}$ moves to the end of the lever arm, but only if $\theta$ is very close to $\theta_{\text {thres }}$, otherwise energy will be lost unnecessary. After initial knee flexion at the beginning of the stance phase the spring must be completely unloaded, to regain knee extension. $\mathrm{P}$ of the unloaded spring can subsequently be reset during the swing phase by decoupling of the upper leg and the lever arm, to reset for the next stance phase. 
This setup can be extended to continuously change $k_{R E}$ without changing the energy in the spring. The relation between $q_{1}$ and $q_{2}$ that must be fulfilled to realize this is described in section 2.3.

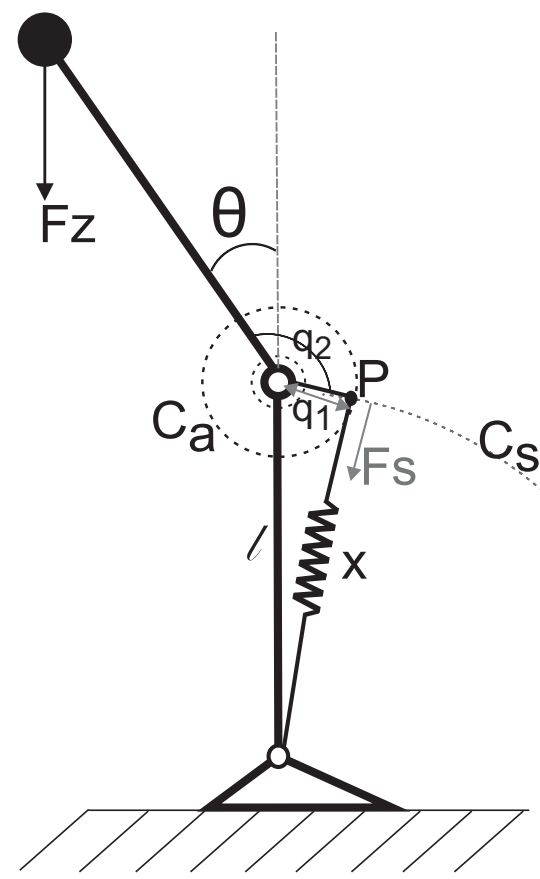

(a)



(b)

Figure 2.1: (a) Example of the energy efficient variable stiffness actuation at a joint using a linear spring. If the length of the lever $\operatorname{arm}\left(q_{1}\right)$ with respect to the joint is changed at a certain $\theta$, the apparent output stiffness changes as well as the moment around the knee. $q_{2}$ is the angle of the lever arm with respect to the upper leg. (b) Possible mechanical setup. The lever arm and upper leg linked during stance. The locks are linked to the lower leg to hold the attachment point $(P)$ in place. The locks will unlock just before $\theta_{\text {thres }}$ is reached and allow $P$ to move to the end of the lever arm.

\section{Modeling energy efficient VSA}

The above concept of changing the rotational stiffness around the knee without changing the energy in the spring, is modeled in Simulink, Matlab (The Mathworks, Inc., Natick, Massachusetts). The rotational stiffness around the knee, equivalent to $k_{R E}$ in the above paragraph is modeled as $k_{0}$, the initial rotational stiffness, and $k_{1}$, the rotational 
stiffness after the switch. Only the specific case is modeled, where the switch is made when the force of the spring is perpendicular to the lever arm. This is done to determine if the controllable spring concept, if it were to be build $100 \%$ efficiently, would be able to control the knee in a reaction to a disturbance during the beginning of the stance phase. During an undisturbed stance phase the knee motion should resemble normal walking. It should also be able to reject a disturbance of the stance phase.

The initial knee angle at heel strike is $\theta_{0}$. The spring exerts a moment where $M_{\text {spring }}=k_{0} \theta$. A controller ensures that $k_{0}$ increases (to $k_{1}$ ) when $\theta$ reaches a certain threshold $\theta_{\text {thres }}$ [82]. The controller initiates this switch from $k_{0}$ to $k_{1}$ at $\theta_{\text {thres }}$ after an assumed time delay $\left(T_{d}\right)$. The time delay causes $\theta$ to increase a little further until the stiffness is effectively changed at $\theta_{\text {change }}$.

In the model it is assumed that at the time of the switch a certain amount of potential energy $\left(E_{k}\right)$ is in the spring. This can also be seen in figure 2.3(a). At the switch $E_{k}=A_{1}=\frac{1}{2} k_{0}\left(\theta_{\text {change }}-\theta_{0}\right)^{2}$, which should be the same after the switch $\left(A_{2}\right.$, fig $\left.2.3(\mathrm{a})\right)$. In this case, $M_{\text {spring }}$ can increase, without changing the energy in the spring, $E_{k}$. This is achieved by increasing $k_{1}$, but also changing the zero moment angle $\left(\theta_{\text {start }_{k 1}}\right)$ of $k_{1}, E_{k}=A_{2}=\frac{1}{2} k_{0}\left(\theta_{\text {change }}-\theta_{\text {start }_{k 1}}\right)^{2}$. In this way $M_{\text {Spring }}$ in the model is increased without changing the energy stored in the spring.

For the second half of the stance phase, the extension phase, a different stiffness $k_{2}$ was assumed in the same way as $k_{0}$ and $k_{1}$, to restore knee extension. The switch from $k_{1}$ to $k_{2}$ was also implemented such that the potential energy at the switch did not change and that at the time $M_{\text {Spring }}$ was zero again, the knee would be back at $\theta_{0}$. If no other energy is added to the system, the knee can only return to $\theta_{0}$. If a fully extended knee is required at push-off and the initial knee angle $\theta_{0}$ is larger than zero, energy needs to be added to the system to restore full knee extension. This can be done by pre-stretching the spring at heel strike. The energy for this can potentially be harvested from previous undisturbed gait cycles, but this is not implemented in the model.

\subsection{Continuous variable stiffness control}

In the following section we will describe the relation between the two degrees of freedom $q_{1}$ and $q_{2}$, defined in figure 2.1. That will allow for 
changing rotational joint stiffness without modification of spring potential energy $\left(E_{k}\right)$.

In rest the spring has a resting length of $x_{0}$, which describes a circle $C_{s}$ (see figure 2.1(a)), with radius $x_{0}$ around the ankle. The lever arm has a variable length $q_{1}$, which describes a circle $C_{a}$, with radius $q_{1}$, around the knee. The point $P$ where the two circles meet is the attachment point of the spring to the lever arm. The lever arm is attached to the upper leg with a variable angle $q_{2}$. Changing the length of the lever arm $q_{1}$ will change the effective rotational output stiffness $\left(k_{R E}\right)$ of the spring, as described by equations 2.1-2.4.

If the energy in the spring is to remain the same when $q_{1}$ and $q_{2}$ are changed the following condition should be satisfied, where $y:=x^{2}$ (figure 2.2):

$$
d y=\frac{\partial y}{\partial q_{1}} d q_{1}+\frac{\partial y}{\partial q_{2}} d q_{2}=0
$$

resulting in the following relation between $d q_{1}$ and $d q_{2}$ :

$$
d q_{2}=-d q_{1}\left(\frac{\frac{\partial y}{\partial q_{1}}}{\frac{\partial y}{\partial q_{2}}}\right)
$$

The length of the spring can be described using the cosine rule (figure 2.2) with:

$$
x^{2}=y=q_{1}^{2}+l^{2}-2 q_{1} l \cos (\alpha)
$$

Where $\alpha=\pi+\theta-q_{2}$. From this we can derive:

$$
\begin{gathered}
\frac{\partial y}{\partial q_{1}}=2 q_{1}-2 l \cos \left(\pi+\theta-q_{2}\right) \\
\frac{\partial y}{\partial q_{2}}=-2 q_{1} l \sin \left(\pi+\theta-q_{2}\right)
\end{gathered}
$$

Combining (2.7), (2.9) and (2.10) results in the following equation for modifying $k_{R E}$ without changing the energy stored in the spring:

$$
d q_{2}=-d q_{1} \frac{q_{1}-l \cos \left(\pi+\theta-q_{2}\right)}{q_{1} l \sin \left(\pi+\theta-q_{2}\right)}
$$

In order to apply this principle of changing rotational stiffness without modifying spring potential energy, an adequate efficient mechanism needs 
to be designed. Currently, such a design is not available. Implementation of this relation using a dynamo and motor is not expected to be adequately efficient. Therefore, an efficient mechanical solution is to be preferred.



Figure 2.2: The triangle between the lower leg, the spring and the lever arm defines angles $\alpha$ and $\beta$.

\subsection{Methods}

The main contribution of the controllable spring is expected during the stance phase of walking from heel strike until push-off, the first $40 \%$ of the gait cycle, for prevention of excessive knee buckling, which could result in a fall. From push-off the knee should allow flexion to accommodate the swing phase. The model consists of a simplified human leg, modeled as a double pendulum with a point mass on top, representing body mass [65, 83-85]. Figure 2.3(b) shows a stick figure representation of the model. Both segments of the pendulum are mass-less with length $l$. The foot is not included, the ankle is modeled as a hinge attached to the floor and the knee is a freely moving hinge controlled by the controllable spring. The initial ankle angle was fixed during all the simulations to a normal ankle angle $(\phi)$ at heel strike of -20 degrees [21]. The mass was given an initial horizontal velocity $\left(v_{\text {init }}\right)$ of $1.2 \mathrm{~m} / \mathrm{s}$ as walking speed [21] for all simulations. All simulations lasted $400 \mathrm{~ms}$, which is around $40 \%$ of the gait cycle at this speed. $k_{0}$ is the initial rotational stiffness, which generates a moment around the knee, repres- 
enting the "normal" initial knee stiffness. The values for the parameters can be found in table 2.1.



(a)

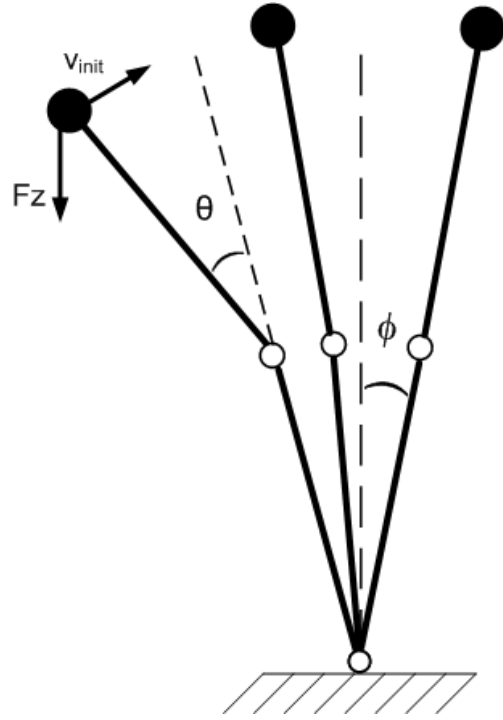

(b)

Figure 2.3: (a) The change in stiffness is initiated when a threshold angle $\left(\theta_{\text {thres }}\right)$ is reached, but the actual change of stiffness takes place at $\theta_{\text {change }}$ due to the time delay in the system. If $\theta_{\text {start }, k_{1}}$ is chosen such that $A_{1}=A_{2}$, no energy needs to be added to the system. $A_{1}$ is the right slanting shaded area and $A_{2}$ is the left slanting shaded area. (b) An example of the motion of the stick figure from heel strike (left) until push off (right) including the initial velocity $\left(v_{\text {init }}\right)$, the positive knee angle $(\theta)$ and the positive ankle angle $(\phi)$.

\subsubsection{Simulations}

The simulations are divided in three parts, first the allowable initial spring stiffness is determined under the normal undisturbed condition during the stance phase. Secondly the controllable spring is tested when there is knee flexion at heel strike, to mimic an unlocked knee, a common disturbance for TFA [86-88]. Finally reflexive control is added to the control loop, by means of a time delay.

\section{Part 1 - Undisturbed stance phase}

First the range of the initial spring stiffness $\left(k_{0}\right)$ during a normal stance 
phase was determined. The initial knee angle at heel strike $\left(\theta_{0}\right)$ was 0 degrees. During the stance phase of normal gait the knee flexes up to 10 degrees [19]. Ideally a prosthetic knee should also flex during this phase to reduce the impact at the residual limb of the TFA. A knee flexion of 3-10 degrees was allowed during stance, for it to resemble a normal stance phase motion. $k_{0}$ was varied from 0 to $5.5 \mathrm{Nm} / \mathrm{deg}$ in steps of 0.1 $\mathrm{Nm} / \mathrm{deg}$. The range of $k_{0}$ that resulted in a knee flexion of 3-10 degrees and for which no switch was needed to allow a normal stance phase, was determined. Only those simulations where the knee was stabilized, were taken into account. This range of $k_{0}$ was used for the second part of the simulations.

During the stance phase a flexion moment around the knee is present due to gravitation. In the proposed knee design the spring allows the knee to be extended again, causing an extension moment. Together this forms the net knee moment. In normal walking the net knee (extension) moment during the stance phase is 50 to $80 \mathrm{Nm}$ [19].

In prosthetic users knee flexion moments between 0.14 up to $0.57 \mathrm{Nm} / \mathrm{kg}$ during prosthetic stance are reported in several studies [64,87-89] and up to $1 \mathrm{Nm} / \mathrm{kg}$ during stair descent [90]. However, a knee extension moment during the prosthetic stance phase in combination with knee flexion is uncommon, because only few knees allow stance flexion and active knee extension. Some studies report an extension moment between 0.47 and $0.69 \mathrm{Nm} / \mathrm{kg}[87,89]$ during normal prosthetic walking. In the model the maximal net knee moment for the undisturbed stance phase $\left(M_{\max }\right)$, was not allowed to exceed $0.5 \mathrm{Nm} / \mathrm{kg}$, which is $40 \mathrm{Nm}$. The top part of table 2.1 shows the parameters used for part 1 of the simulations.

\section{Part 2 - Disturbed stance phase}

In the second part a disturbed stance phase was modeled. Two larger initial knee angles of 8 and 16 degrees were used, to simulate a person landing on an unlocked knee at heel strike, a common disturbance in transfemoral prosthetic walking. From this we determined if the controllable spring was still able to control the disturbed knee, with the spring settings of $k_{0}$ found in the first part. When knee flexion exceeded $8^{\circ}\left(\theta_{\text {thres }}\right)$, the spring stiffness was changed from $k_{0}$ to $k_{1}$, with a time delay $\left(t_{d}\right)$ of $25 \mathrm{~ms}$ [82]. This time delay modeled the time required for the stiffness to be actually switched.

For the range of suitable $k_{0}$ found from part $1, k_{1}$ was varied (from $1-30 \mathrm{Nm} / \mathrm{deg}$, in steps of $1 \mathrm{Nm} / \mathrm{deg}$ ) to determine if the controllable 
spring could stabilize the knee after a disturbance. Hereby stabilized is described by a maximal knee flexion of $30^{\circ}$ and a maximal net knee moment of $160 \mathrm{Nm}$. A larger knee angle was considered to be a fall. Bellmann et al. [90] found that the C-leg under weight-bearing load collapsed if the knee angle exceeded $30^{\circ}$. Especially at the beginning of the stance phase a larger knee angle might cause the center of mass to move too far away from the center of pressure, that it can be considered a fall. The maximal net knee moment (flexion and extension) allowable at the knee is difficult to determine. In the study by Blumentritt et al. [67] knee extension moments around $120 \mathrm{Nm}$ were measured during landing on foreign objects and stumbling, in another study a knee extension moment of $157 \mathrm{Nm}$ was measured during a fall [91]. We therefore set the maximal net knee extension moment at $160 \mathrm{Nm}$ for part 2 (\& 3). This knee moment is only acceptable for those stance phases where a disturbance is modeled, a high knee moment is in this case preferred to fall. For the model a $k_{2}$ was used to extend the knee back to $\theta_{0}$. From the moment the knee was stabilized, at the largest knee flexion angle, $k_{1}$ is switched to $k_{2}$, in a similar way as from $k_{0}$ to $k_{1}$ (see also figure $2.3(\mathrm{a})) . k_{2}$ was calculated such that at the second switch the energy in the spring remained the same, the knee was extended back to $\theta_{0}$, and all energy in the spring is used ( $M_{\text {Spring }}$ is back to zero). Table 2.1 shows all the parameters used.

\section{Part 3 -Reflexive control of a disturbance}

In the third part we modeled a disturbed stance phase with reflexive control and predicted whether the reflexive user control loop will be fast enough to contribute to effective disturbance rejection, given the expected delay of the control loop. In the model the reflexive control was modeled as one large time delay of $105 \mathrm{~ms}$. This time delay consisted of four main parts, the time required for disturbance detection $(50 \mathrm{~ms})$, triggering of the artificial reflex and the subsequent time delay until EMG can be detected (40 ms), EMG detections and decision (10 ms) and the control of the knee (5 ms) [4, 5, 76-79,92]. All separate time delays are based on delays measured in previous studies and we chose the smallest available time delay for each part of the loop. We modeled the smallest disturbance from Part 2 , the $\theta_{0}=8^{\circ}$ with a $k_{0}$ of $5 \mathrm{Nm} / \mathrm{deg}$ (max. from Part 1), and a $k_{1}$ between 5 and $30 \mathrm{Nm}$. All boundary conditions were similar to part 2 . 


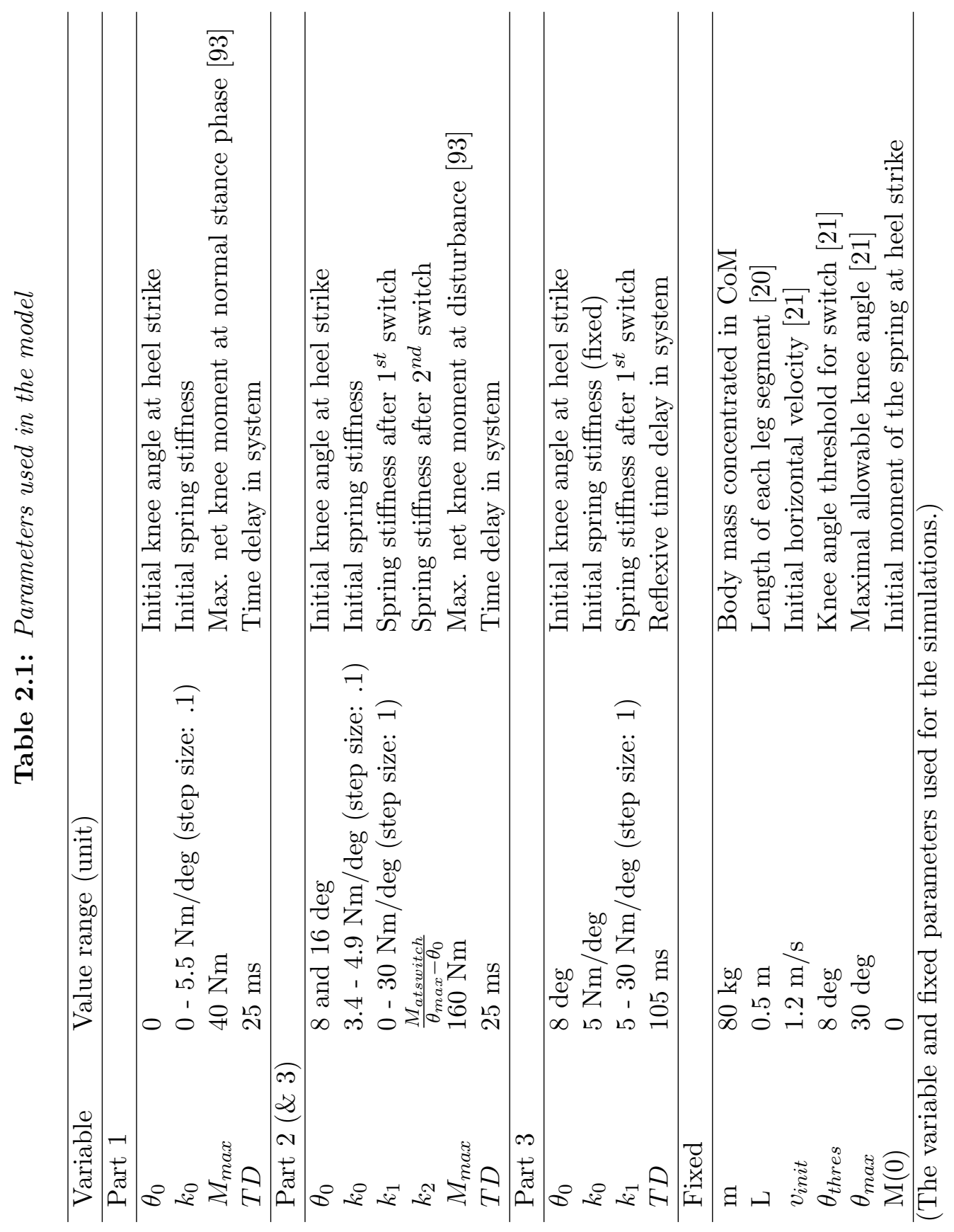




\subsection{Results}

Figure 2.4 shows an example of one simulation of the disturbed stance phase, where $\theta_{0}$ is 8 degrees and $t_{d}$ is $25 \mathrm{~ms}\left(k_{0}=3.6 \mathrm{Nm} / \mathrm{deg}, k_{1}=8\right.$ $\mathrm{Nm} / \mathrm{deg}, k_{2}=6.8 \mathrm{Nm} / \mathrm{deg}$ ). The stick figure motion during one trial is shown, together with the spring moment as a function of time and knee angle, and the knee angle as function of time.

$\theta$ is 8 degrees at heel strike and the switch is immediately initiated, the time delay causes another 5-6 degrees of flexion, after which the spring changes to $k_{1}$. At the point where $\theta$ reaches its maximum, the spring changes to $k_{2}$ for the knee to return to its original angle of 8 degrees. The potential energy in the spring does not change as the stiffness changes from $k_{0}$ to $k_{1}$ and from $k_{1}$ to $k_{2}$. This is also shown by the graph with $M_{\text {spring }}$ as function of $\theta$ (fig. 2.4(d)) which has a similar shape as the graph of figure 2.3(a).

\subsubsection{Part 1 - Undisturbed stance phase}

Figure 2.5 a shows the results of the simulation with a range of $k_{0}$ to find the allowable combination for a "normal" stance phase. For those simulations where $k_{0}$ was smaller than $3.4 \mathrm{Nm} / \mathrm{deg}$ the knee collapsed and was not stabilized.

$k_{0}$ larger than $3.4 \mathrm{Nm} / \mathrm{deg}$ was able to stabilize the knee, whereby the net knee moment did not exceed the normal stance phase moment $(40 \mathrm{Nm})$. For a $k_{0}$ between 3.4 and $3.9 \mathrm{Nm} /$ deg knee flexion after heel strike was 8 to 3 degrees which resembles normal walking [19,21], $k_{0}$ of $4.0 \mathrm{Nm} / \mathrm{deg}$ or larger did not reach the desired amount of knee flexion. For $k_{0}>$ $5.5 \mathrm{Nm} /$ deg the maximal knee flexion does not decrease any further and remains around $1.5^{\circ}$. Therefore a $k_{0}$ between 3.4 and $3.9 \mathrm{Nm} / \mathrm{deg}$ is the desired initial knee stiffness for non-disturbed knee patterns, a larger $k_{0}$ is possible but undesirable.

\subsubsection{Part 2 - Disturbed stance phase}

Figure 2.6 shows the results of the simulations where the $\theta_{0}$ was increased to 8 and 16 degrees. The time delay was $25 \mathrm{~ms}$ and $k_{0}$ was varied between 3.4 to $5.5 \mathrm{Nm} / \mathrm{deg}$. For the $\theta_{0}$ of $8^{\circ}$ the knee angle and knee moment remained within the limits of $30^{\circ}$ and $160 \mathrm{Nm}$ respectively for $k_{1} \geq 7$ $\mathrm{Nm} /$ deg. For the $\theta_{0}$ of $16^{\circ}$ the knee angle did not exceed the maximal knee angle for $k_{1}>13 \mathrm{Nm} / \mathrm{deg}$. For $\theta_{0}$ of $16^{\circ}$, the maximum knee extension moment satisfied the $160 \mathrm{Nm}$ criterium only marginally for $k_{1}$ between 18 and $24 \mathrm{Nm} / \mathrm{deg}$ and $k_{0}>4.3 \mathrm{Nm} / \mathrm{deg}$. 



Figure 2.4: An example of a disturbed stance phase. (a) The knee angle over time from heel strike until push-off. (b) Stick figure representing the leg kinematics from heel strike until just before push-off. (c) The spring moment as function of time. (d) The spring moment as function of the knee angle. The solid line shows the first phase in $k_{0}$, the dashed line the second phase after the switch to $k_{1}$ and the dotted line the final phase in $k_{2}$. At $25 \mathrm{~ms}$ the rotational spring stiffness changes from $k_{0}$ to $k_{1}$, consequently increasing $M_{\text {spring. At }} 110 \mathrm{~ms}$ the knee reaches maximal knee flexion (23.8 ) and the spring stiffness is changed from $k_{1}$ to $k_{2}$, $M_{\text {spring }}$ decreases and the knee is extending again. $\left(\theta_{0}=8^{\circ}, T_{d}=25\right.$ $m s, k_{0}=3.6 \mathrm{Nm} / \mathrm{deg}, k_{1}=8 \mathrm{Nm} / \mathrm{deg}, k_{2}=6.8 \mathrm{Nm} / \mathrm{deg}, \theta_{\text {start }, k_{1}}=$ $\left.9.5^{\circ}\right)$.

\subsubsection{Part 3 - Reflexive control of a disturbance}

From Parts 2.5.1-2.5.2 we found that a $k_{0}$ of $5.5 \mathrm{Nm} / \mathrm{deg}$ was the maximal rotational stiffness whereby no switch was needed for a $\theta_{0}=0^{\circ}$. We used this maximal initial stiffness for modeling the reflexive loop. Figure 2.7 shows the results from the model using the time delay of 105 ms, which we assumed to be minimal for a user reflexive control loop. There was no $k_{1}$ which satisfied the conditions that the knee angle and knee moment both remained within the boundary conditions. 




Figure 2.5: Results for the "normal" stance phase simulations, where $\theta_{0}$ was 0 degrees. [-] extension moment ( $\left.\mathrm{Nm}\right)$, [- -] maximal knee angle (degrees). At the $k_{0}$ where the knee angle is stabilized, $(3.4 \mathrm{Nm} / \mathrm{deg})$ the extension moment is also below its maximal allowed moment of $40 \mathrm{Nm}$, from $3.8 \mathrm{Nm}$ the max. knee angle is below $2^{\circ}$. For these simulations only $k_{0}$ was varied between 0 and $5.5 \mathrm{Nm} /$ deg in steps of $0.1 \mathrm{Nm} / \mathrm{deg}$.



Figure 2.6: In black $\theta_{0}$ is $8^{\circ}$, in gray the results for $\theta_{0}$ is $16^{\circ}$. [-] average maximal extension moments. [- - ] average maximal knee angle. The light grey areas represent the range of maximal extension moments for different $k_{0}$ (from 3.4- $5.5 \mathrm{Nm} / \mathrm{deg}$ ). The horizontal line represents the maximal allowable knee angle. The dotted horizontal line represents the maximal allowable knee moment. For the larger initial knee angle $\left(16^{\circ}\right)$ the maximal knee moment only remains below $160 \mathrm{Nm}$, for $k_{0}>$ $4.3 \mathrm{Nm} / \mathrm{deg}$. (bottom part of the light grey area) 


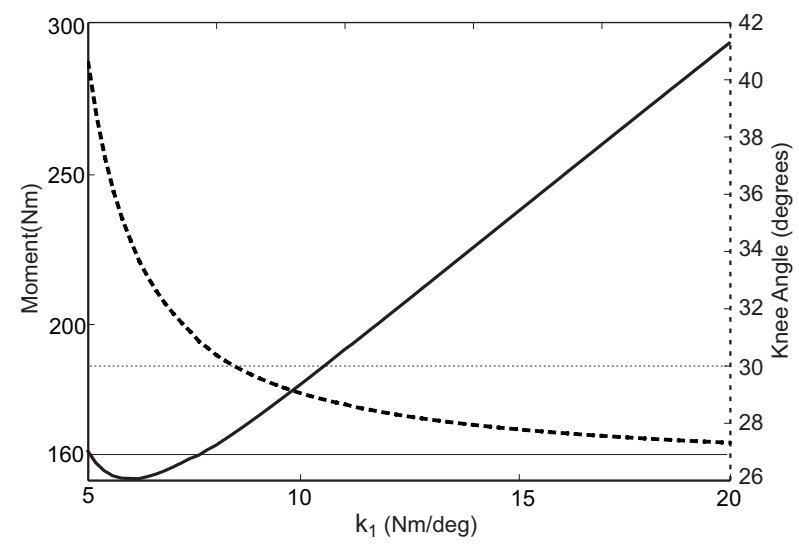

Figure 2.7: The solid black line represents the maximal extension moment and the dashed line represents maximal knee angle, both for $\theta_{0}$ is $8^{\circ}, k_{0}$ of $5.5 \mathrm{Nm} / \mathrm{deg}$, a time delay of $105 \mathrm{~ms}$ and different $k_{1}$. The solid horizontal line represents the maximal allowable knee moment. The dashed horizontal line represents the maximal allowable knee angle. No configuration was found whereby all boundary conditions were met.

\subsection{Discussion}

The goal of the study was to introduce a new method to control a prosthetic knee in an energy efficient manner. We intended to predict the usability of energy efficient variable stiffness control in upper leg prostheses, in a modeling study. In addition we predicted if reflexive user control is possible using a VAS controlled prosthetic knee. The results of the simulations showed that the controllable spring concept as implemented in the model is capable of stabilizing the knee joint during undisturbed and disturbed stance phases within identified limits. For a extended knee at heel strike no change of stiffness was needed. Disturbances of the initial knee angle at heel strike could be rejected by increasing the stiffness.

Our model predicted that the total time delay of $105 \mathrm{~ms}$ is too large to correct a disturbance. However the boundary conditions largely determine the final outcome. It appears that if the allowable knee flexion is increased from 30 to 32 degrees, the boundary conditions will be met. Although these boundary conditions are carefully determined, further research is needed to determine if they can be widened. Some of the constituting parts of the time delay, for instance of EMG and disturb- 
ance detections, take time to detect especially if a high specificity and sensitivity is required. It is unlikely that these detection time delays will decrease in the future. It should be noted that user feedback and control may be useful under less time-critical conditions than rejection of a disturbance as modeled.

The modeled disturbance was one of many possible disturbances. In addition only one switch to increase the stiffness could be performed in the model to correct the disturbance. Chapter 2 also described the possibilities of continuously adapting the stiffness of the knee, to the needs of the user. This continuous type of variable stiffness actuation needs further research.

\subsubsection{Conceptual considerations}

Although the model is a simplified version of the normal situation and only simulates the stance phase of gait, our model predicts that the controllable spring can be useful when implemented. During the stance phase the knee needs to be controlled by the spring whereas during the swing phase the stiffness around the knee can be relatively low to allow the knee to flex for ground clearance and extend to prepare for heel strike. Energy absorption in the spring during the swing phase, as shown by Unal et al. [14] could be an additional implementation. The knee may also be locked at heel strike if it has reached zero degree flexion. This will prevent any flexion after heel strike and is the safe option, which is often the case in current prosthetic knees. We preferred the option of a slight flexion in the stance phase to better represent normal walking and reduce the impact during weight acceptance subsequent to heel contact. The simulations showed that this can be realized in combination with the rejection of inadequate knee extension at heel contact. The knee moment during normal stance resembles that of other studies, even with some degree of stance knee flexion [64,87-90].

The energy efficiency of this concept lies in the preservation of potential energy whilst increasing the output moment of the spring [61]. Together with this the spring allows the knee to be extended again to the original angle after being flexed, in contrast to a damper. It should be noted that current intelligent prostheses with a controlled knee damper can stop knee flexion when the knee is not adequately extended at heel contact, but are not able to help extend the knee subsequently.

We believe that the simple version, using a fixed $q_{2}$ can be built mech- 
anically and (almost) completely energy efficient. The described implementation may benefit from a small amount of energy taken from the spring to increase the moment arm in order to increase the rotational stiffness if required. This can be implemented if the stiffness is changed just before the knee angle reaches the knee angle at which the moment arm is perpendicular to the direction of spring force.

Although in theory the stiffness can be controlled without adding energy, in practice this is not expected to be the case, since a mechanism for changing rotational stiffness without changing the potential energy of the spring will have losses. At the moment it is unsure if this will be more energy efficient than the current controllable dampers. The dynamic characteristics of this potential mechanism are also important and is also an issue that needs to be addressed, if this becomes too slow energy will be lost and as we saw in the reflexive simulation, the model may become unstable.

The implementation of the spring for continuous switching, as described in the 2.3, will however need some energy and still some thought. It will need a micro-controller and a motor to change $q_{1}$ and $q_{2}$. The mechanical energy needed to change $q_{1}$ is the same but negative to the energy needed to change $q_{2}\left(F_{1} \dot{q}_{1}=-M_{2} \dot{q}_{2}\right)$. If an efficient way can be found to couple a motor and a dynamo, the motor to change $q_{1}$ can at the same time load the dynamo. However, this solution would required transfer of energy from the mechanical to the electrical domain and back, which is in practice not efficient. Therefore, a mechanical solution to change $q_{1}$ and $q_{2}$ simultaneously in a continuous manner, without changing the total potential energy, is preferred. However, we have not been able to design an adequate mechanism for this purpose until now.

Efficiency in potential energy does not necessarily mean that metabolic energy consumption by the user will reduce when it is implemented in an upper leg prosthesis. The controllable dampers have already proven that prosthetic walking becomes more energy efficient [94], even though they do not actively extend the knee. The use of the spring allows active knee extension during the stance phase, which may also contribute in reducing the metabolic energy consumption, but this is still to be evaluated [83]. Farber et al. [81] found that the energy cost to the user decreased up to $35 \%$ using a linear non adjustable spring. 


\subsubsection{Modeling considerations}

The controllable spring was able to stabilize the disturbance of an unlocked knee even within the boundaries of the maximal knee moment and knee flexion. For $\theta_{0}$ of $16^{\circ}$, increasing the knee stiffness was unsuccessful, within the desired range of $k_{0}$, due to the large knee extension moment.

The modeling results are highly dependent on the limits set to the knee angle and knee moment, especially for the disturbed stance phases. These limits are however hard to determine from literature and are highly dependent on several factors such as the physical condition of the prosthetic user and the residual limb length. From literature both boundaries of 30 degrees knee flexion and $160 \mathrm{Nm}$ knee moment seem to be acceptable and favorable compared to a fall [67,91]. However there are many more disturbances possible with a prosthetic knee, which need to be examined to determine the safety of the controllable spring when implemented [24,67].

In the current model a linear spring was used. The results may improve if a non-linear spring is used, which gains a higher spring stiffness at a larger knee angle. If such a spring is used, switching to increase the rotational stiffness may not be necessary anymore. However, such a construction will not allow to control the spring characteristics depending on the circumstances, as current intelligent knee prosthesis also do with variable damping(Genium, Otto Bock; Rheo knee, Ossur).

The actual time delay will also affect the results. $25 \mathrm{~ms}$ is an estimate for signal processing and making the switch. Current prosthesis have an update rate between $100 \mathrm{~Hz}$ (Genium, Otto Bock) and $1000 \mathrm{~Hz}$ (Rheo knee, Ossur), which would leave between 15 and $24 \mathrm{~ms}$ to make the switch. The actual time delay will however depend on the practical implementation of the system, which is still under construction.

For the simulations a fixed threshold of 8 degrees was set to determine if the stiffness needed to be changed, based on knee flexion during a normal stance phase $[19,21,82]$. Making the switch at a smaller knee angle may require a lower $k_{0}$ and $k_{1}$, but will also have its effect on the undisturbed knee patterns. A smaller threshold will allow less flexion after heel strike and will require a change of stiffness more often. Another option is to use a different threshold altogether. Knee angle may not be the ideal quantity to monitor, the angular velocity of the knee 
may be a better indicator of a disturbance, either or not in combination with the knee angle. Rather than using a threshold the stiffness could also be continuously controlled using $q_{1}$ (and $q_{2}$ ) as described in 2.3.

\subsection{Conclusions}

In this study we proposed to use energy efficient variable stiffness actuation to control the rotational stiffness around a knee joint. Controlling the rotational stiffness allows knee flexion in the stance phase and active knee extension. Excessive knee flexion can be corrected by increasing the rotational knee stiffness without or with minimal change of spring energy. This only holds if a linear spring is used of which the knee moment arm is changed at the instance when the arm is perpendicular to the spring force or just before. Changing joint stiffness without modifying spring potential energy can in principle be implemented continuously, just like continuous controllable damping in current intelligent prostheses. An efficient mechanism for this purpose is, however, yet to be designed. From the model we concluded that the time delay representing the reflexive user control and feedback loop, is too large to safely control a VSA controlled prosthetic knee during a disturbance. 


\section{Part II}

\section{Voluntary control of upper leg prostheses}





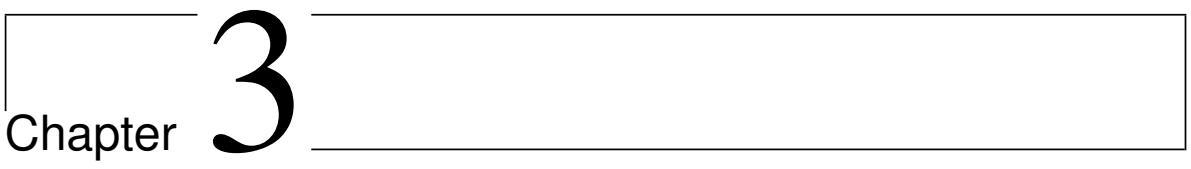

\section{EMG of transfemoral amputees and controls during level walking ${ }^{1}$}

Only few studies have looked at electromyography (EMG) during prosthetic gait. Differences in EMG between normal and prosthetic gait for stance and swing phase were never separately analyzed. These differences can give valuable information if and how muscle activity changes in prosthetic gait. In this study EMG activity during gait of the upper leg muscles of six transfemoral amputees, measured inside their own socket, was compared to that of five controls. On and off timings for stance and swing phase were determined together with the level of co-activity and inter-subject variability. Gait phase changes in amputees mainly consisted of an increased double support phase preceding the prosthetic stance phase. For the subsequent (pre) swing phase the main differences were found in muscle activity patterns of the prosthetic limb, more muscles were active during this phase and/or with prolonged duration. The overall inter-subject variability was larger in amputees compared to controls. The changes in muscle activity may be the consequence of changes in walking strategy, but are likely also aimed to increase socket fitting.

\footnotetext{
${ }^{1}$ Published in: Journal of NeuroEngineering and Rehabilitation 10:87 2013
} 


\subsection{Introduction}

During rehabilitation transfemoral amputees learn to adapt their gait pattern to walk with a prosthesis. Several of these adaptations are already known. During gait the stance phase of the amputated limb shortens compared to that of the intact limb. Therefore the swing phase is longer for the amputated limb. The double support phase elongates when the prosthetic limb becomes the stance limb and shortens when the intact limb becomes the stance limb $[24,26]$. The comfortable walking speed of prosthetic walkers is also lower than in normal walking $[26,27,95,96]$. Kinematic data shows that transfemoral amputees lack plantar flexion power (push-off) at the prosthetic side. To facilitate forward propulsion they increase the work by the hip joint at the prosthetic and intact side and the plantar flexors at the intact side $[3,95,96]$. Eccentric work at the hip of the intact side decreases with respect to normal gait [96]. Joint power during concentric knee extension increases for the intact side, with respect to normal walking [95].

However, little research has been performed on electromyography (EMG) during amputee gait. EMG of residual limb muscles of TFA may give valuable information on adaptations besides those that can already be found using the kinetic and spatio-temporal data [97]. Some studies report increased and prolonged muscle activity in amputees during gait $[3,27,98]$. Bae et al. [98] concluded that the co-activation of the upper leg muscles of the intact limb in amputees was larger than in controls. Hong and Mun [38] found that during gait the muscle activity of residual limb muscles in TFA is correlated to the socket pressure. If EMG patterns are different from that of controls this might indicate specific adaptations of amputees. Muscle activity per phase (stance and swing) can give more insight in the changes in the muscle activity patterns, how they change compared to normal walking and in the adaptations amputees make when walking with a prosthesis, besides kinematic changes.

In the current study we focus on muscle activity during the stance and swing phase of prosthetic gait. Do the muscle activity patterns of the prosthetic limb change and how do they change for the stance and swing phase, compared to normal gait? We intended to have as little interventions to the prosthesis and the subsequent walking pattern as possible. Therefore we measured EMG inside the socket, without modifications, of six amputees and compared this to data of five controls. Previous studies have shown that it is possible to measure EMG with acceptable quality inside the socket of amputees [27,99]. 
From this data we determined if the timings of the muscle activity changed with respect to the different phases of gait compared to normal gait. We hypothesized that the general EMG patterns during walking are comparable to those in controls, but we expected to find differences related to specific adaptations in amputees. Three muscles at the contralateral lower leg were also measured to determine the adaptations at the intact lower leg. We determined how the inter-subject variability of amputees compares to that of controls. Spatio-temporal and kinematic data were also measured to determine the gait phases and to compare the results to previous studies.

\subsection{Methods}

\subsubsection{Participants}

Eleven healthy subjects participated in the study, five controls and six unilateral amputees. All subjects were recruited between April and July 2011. Of the amputees there were three transfemoral amputees (TFA) and three through knee amputees (TKA). An overview of the amputees can be found in Table 3.1. Inclusion criteria were: have a unilateral TFA or TKA regardless of the reason for amputation; be between 18 and 70 years old; be a prosthetic user able to walk independently with or without a walking aid (K-level 2, 3 and 4).

The controls were on average aged 23 (range 21-27) and had no history of lower leg injuries, neurodegenerative diseases or any skin conditions. An informed consent was obtained before the experiments, and the study was approved by the local Ethics Committee. 


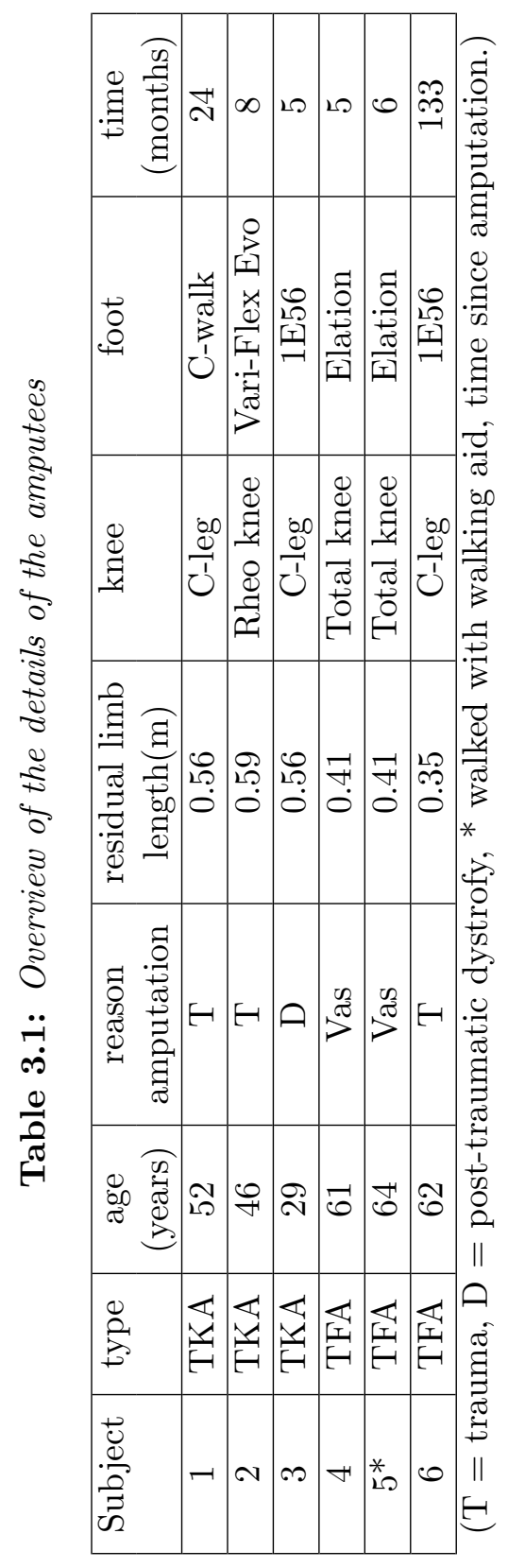




\subsubsection{Measurements}

EMG recording was performed on eight upper leg muscles in all subjects: $\mathrm{m}$. gluteus maximus (GMa), m. gluteus medius (GMe), m. tensor fasciae latae $(\mathrm{TFL}), \mathrm{m}$. rectus femorus $(\mathrm{RF}), \mathrm{m}$. vastus lateralis $(\mathrm{VL})$, $\mathrm{m}$. biceps femoris $(\mathrm{BF}), \mathrm{m}$. semitendinosis (ST), m. adductor magnus (Add). In amputees these were measured on the residual limb, in controls these muscles were measured at one limb, which was alternated between dominant and non-dominant limb. For amputees and controls this limb will be called the "prosthetic limb" and "mimicked prosthetic limb" respectively.

At the contralateral lower limb three more muscles were measured, the $\mathrm{m}$. tibialis anterior (TA), m. gastrocnemius medialis (GaM) and the $\mathrm{m}$. soleus (Sol). For amputees and controls this limb will be called the "intact limb" and the "mimicked intact limb" respectively.

Electrodes were placed according to the SENIAM standards [100], by an experienced physical therapist. For the amputees the locations were approximated, but EMG was checked prior to the measurements by selective contraction of the muscle [100]. On each muscle two self adhesive electrodes (Ambu, BRS) were placed as closely together as possible. EMG measurements were performed with a 16 bipolar channel Portisystem (TMSi, Oldenzaal, the Netherlands) at a sample frequency of $2048 \mathrm{~Hz}$, no pre-filtering was applied.

Footswitches, placed mid-heel and under the first metatarsal head of each the foot, gave information about initial contact and initial swing. Footswitch data was registered with the Porti-system.

Kinematic data were measured $(100 \mathrm{~Hz})$ using inertial sensors from Xsens (Xsens, Enschede, the Netherlands), with 3D accelerometers, 3D gyroscopes and 3D magnetometers. Two inertial sensors were placed at the upper and lower (mimicked) prosthetic limb. Subjects wore their own low-heeled shoes.

To synchronize EMG, footswitches and inertial sensors a synchronization pulse (sync) was given at the start and end of each measurement which was visible in all data sets.

\subsubsection{Procedures}

For the experiments the subjects were asked to walk at a self selected walking speed. After data recording was started, the sync was pressed and subjects started walking. After five steps they were asked to stop, turn around, wait 2-3 seconds, press the sync and walk back; this constituted one trial. Four trials were performed in all subjects. 


\subsubsection{Data analysis}

From the footswitch data the timings of initial contact (IC), terminal stance, initial swing and loading response of each limb were determined [22]. Foot switches were used to extract the spatio-temporal information. Full strides were cut from the EMG and inertial sensor data, from IC to IC of the (mimicked) prosthetic limb. Strides with gait initiation or termination were excluded. All strides per subject were aligned at IC of the (mimicked) prosthetic limb.

\section{Inertial sensor data}

The inertial sensors express their data in the sensor coordinate system $(S)$. Two calibration exercises were performed to convert this data to the body coordinate system $(F)$, using the rotation matrix $\left(R_{f_{s}}\right)\left(F=R_{f s} S\right)$. In short the calibration of the lower leg was as follows. The subject stood upright, whereby the body z-axis equals the gravity vector which can be described in the sensor coordinate system. Subsequently the subject flexed the knee five times to about 90 degrees, where the knee is the body $y$-axis, allowing this axis to be defined in the sensor coordinate system. The $\mathrm{x}$-axis is subsequently obtained by a cross product of the $\mathrm{y}$ and z-axis. For the amputee group the knee was flexed by the researcher. A similar procedure was followed for the upper leg sensor. Here the squat was used as calibration exercise for controls and for amputees the upper leg was lifted forwards [101,102].

The calibrated data was subsequently low-pass filtered at $10 \mathrm{~Hz}$ with a second order, butterworth filter. From the calibrated inertial sensor data the knee angle, hip adduction and abduction are calculated using accelerometer and gyroscope data by the method described by Watanabe et al. [103].

\section{EMG data}

EMG data was first high pass filtered at $10 \mathrm{~Hz}$ and subsequently low pass filtered at $500 \mathrm{~Hz}$, both with a second order butterworth filter. In Figures 3.2, 3.3, 3.4, and 3.5 the ensemble averages of all amputees and controls separately are provided, including the raw and filtered data of one subject, of one trial for all muscles. In Figure 3.2 an example of filtered data is presented. For on and off detection the data was rectified and integrated (IA) in a window of 20 samples, a post processor of 4 windows was used. The threshold for on/off detection was determined 
per muscle. A period of rest activity was selected, and the mean IA value of this period plus three times the standard deviation was used as threshold for onset and termination of muscle activity [104-106]. For each muscle, each stride and each subject the on/off timings were calculated. These timings were averaged per subject, to get the on and off timings per muscle, per subject.

The stance and swing phase of the (mimicked) prosthetic limb were calculated per subject and expressed as percentage of the total stride time. Using the average muscle on/off timings per subject, we subsequently calculated for which percentage of the stance or swing phase the muscles were active. These were subsequently averaged for the controls and the amputees. Differences between controls and amputees were analyzed using a Kruskal-Wallis test. The level of alpha was set at 0.05.

The inter-subject variability of the EMG data was determined using the variance ratio (VR) for each subject and muscle for the stance and the swing phase $[106,107]$. The VR is the variance of the data between gait cycles normalized to the total variance, whereby 0 indicates a low variance and 1 a high variance. Differences between the controls and amputees were analyzed using the Mann-Whitney-Wilcoxon test [106]. The standard error of the mean (SEM), was calculated using $S E M=$ $\frac{S D_{\% \text { on } / \text { offtime }}}{\sqrt{N}}$, where $\mathrm{N}$ is the number of subjects per group [108].

\subsection{Results}

\subsubsection{Kinematic data}

In Table 3.2 the average duration of a stride and the different gait phases in percentages of a stride are presented. A shift of all phases can be seen for amputees, figure 3.1. For amputees the relative duration of the stance phase of intact limb, the prosthetic swing phase and the (first) double support phase before the prosthetic single stance phase are significantly increased compared to controls. The (second) double support phase of amputees before the prosthetic swing phase, is shortened but not statistically significant. Compared to the total stance phase, this "second" double stance phase is equal for both controls and amputees (15\%). No differences were found between TFA and TKA, nor between mechanical and micro-processor-controlled (MPC) knees. 


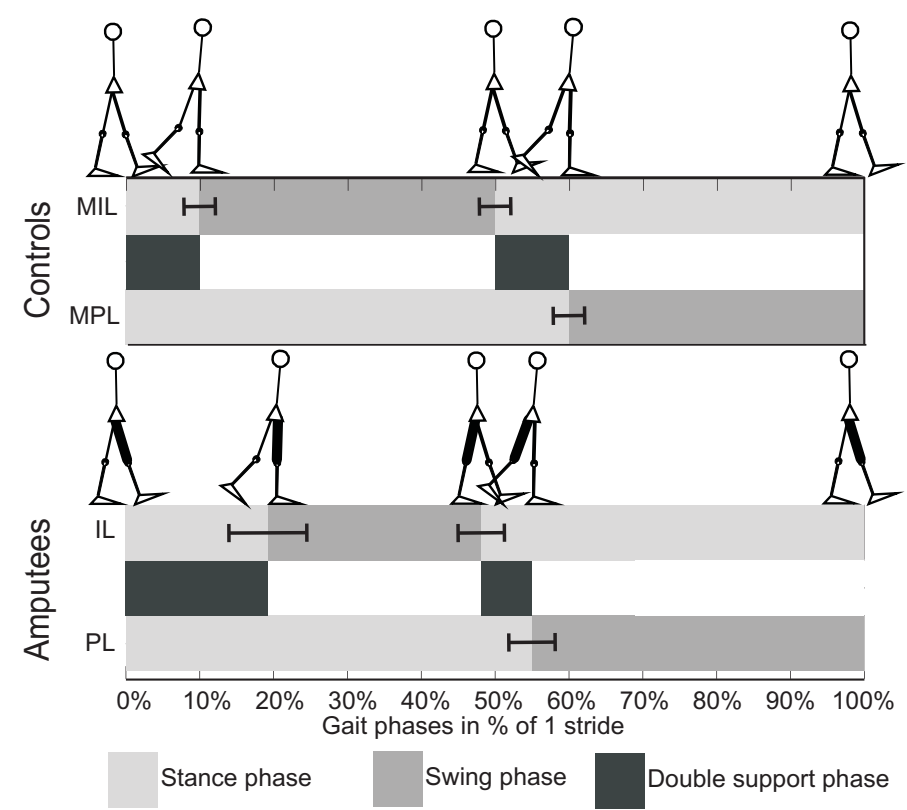

Figure 3.1: Gait phases for controls and amputees, as percentages of one full stride. In black the swing phase, in light grey the stance phase and in dark grey the double support phases. The whiskers give one SD. $(M) I L=$ (mimicked) intact limb, $(M) P L=$ (mimicked) prosthetic limb.

\section{Joint rotations}

Table 3.3 shows the movement patterns around the hip and knee joints. Hip adduction and abduction are significantly reduced in amputees compared to controls. Knee flexion during stance as well as swing is also significantly reduced in amputees. No differences were found between TFA and TKA, nor were they found between mechanical and MPC knees. 
Table 3.2: Gait phases

\begin{tabular}{|c|c|c|c|}
\hline Phase & $\begin{array}{c}\text { Controls } \\
\text { value (SD) }\end{array}$ & $\begin{array}{c}\text { Amputees } \\
\text { value (SD) }\end{array}$ & statistical note \\
\hline Stride duration & $1256 \mathrm{~ms}(72)$ & $1468 \mathrm{~ms}(307)$ & $\mathrm{NS}$ \\
\hline Total stance (M)PL & $61 \%(2)$ & $55 \%(9)$ & $\mathrm{C}>\mathrm{A} \mathrm{p}=.010$ \\
\hline Total stance (M)IL & $60 \%(3)$ & $71 \%(6)$ & $\mathrm{C}<\mathrm{A} \mathrm{p}=.008$ \\
\hline Swing (M)PL & $39 \%(2)$ & $45 \%(3)$ & $\mathrm{C}<\mathrm{A} \mathrm{p}=.010$ \\
\hline Swing (M)IL & $40 \%(3)$ & $29 \%(3)$ & $\mathrm{C}>\mathrm{A} \mathrm{p}=.008$ \\
\hline DLS (M)PL & $11 \%(1)$ & $20 \%(9)$ & $\mathrm{C}<\mathrm{A} \mathrm{p}=.045$ \\
\hline DLS (M)IL & $10 \%(3)$ & $7 \%(3)$ & NS \\
\hline
\end{tabular}
intact limb, $(\mathrm{M}) \mathrm{PL}=($ mimicked $)$ prosthetic limb, SLS $=$ single limb support, DLS = double limb support.) 


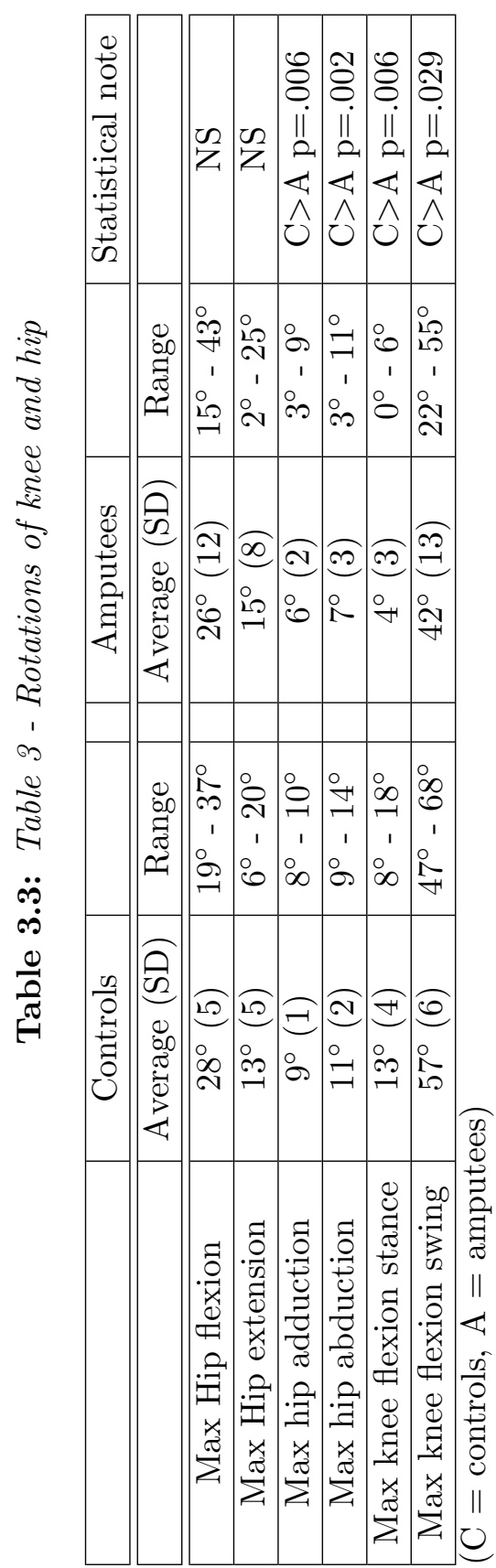




\subsubsection{EMG data}

In Figure 3.2 a sample trial of EMG measured inside (upper leg) and outside (lower leg) the socket is provided of one subject. Both EMG measured inside and outside the socket shows to be of similar quality, without motion artifacts. 24 complete steps were measured in each subject, per subject at least 20 steps were included in the analysis. No steps were excluded from the controls. From the amputee data four subjects showed motion artifacts (see example in Figure 3.3) in maximally three trials in one or more muscles. In one other amputee four trials were excluded due to missing footswitch data. Figures 3.4 and 3.5 show the ensemble averages of each of the controls and amputees respectively. Figure 3.6 shows the timings of the upper leg muscles of the (mimicked) prosthetic limb for amputees and controls as percentages of the stance and swing phases of the (mimicked) prosthetic limb. Data of the lower leg muscles are from the contralateral limb. 

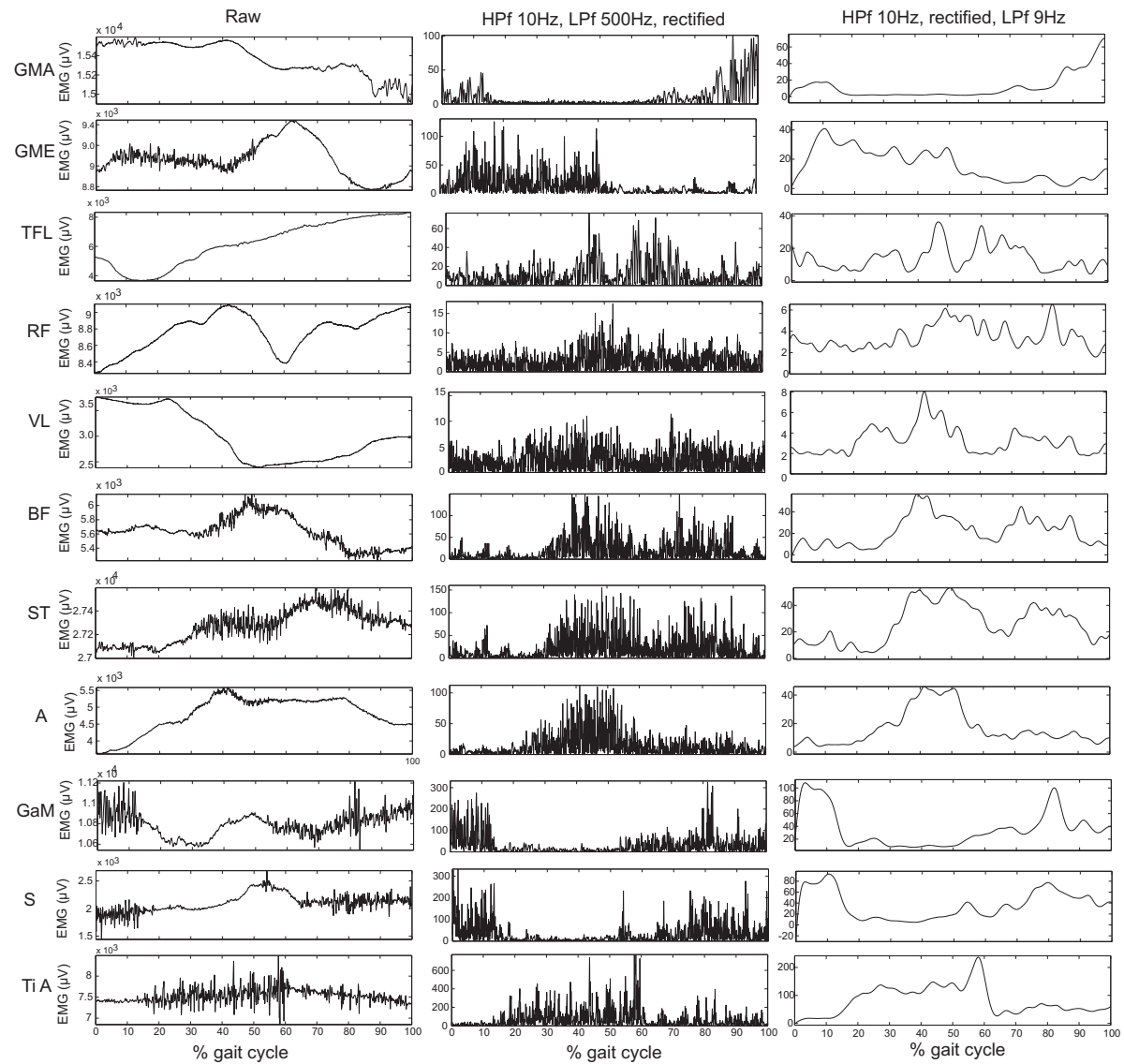

Figure 3.2: An example of filtered activity of all upper and lower leg muscles, of one subject during one trial. The row on the left shows the raw data of the trial during one gait cycle. The middle row shows the high pass filtered (HPf), rectified and low-pass filtered (LPf) data of the same trial and the right hand row shows the linear envelope $\mathrm{HPf}$ at $10 \mathrm{~Hz}$, rectified and LPf at $9 \mathrm{~Hz}$. 
Biceps Femoris EMG
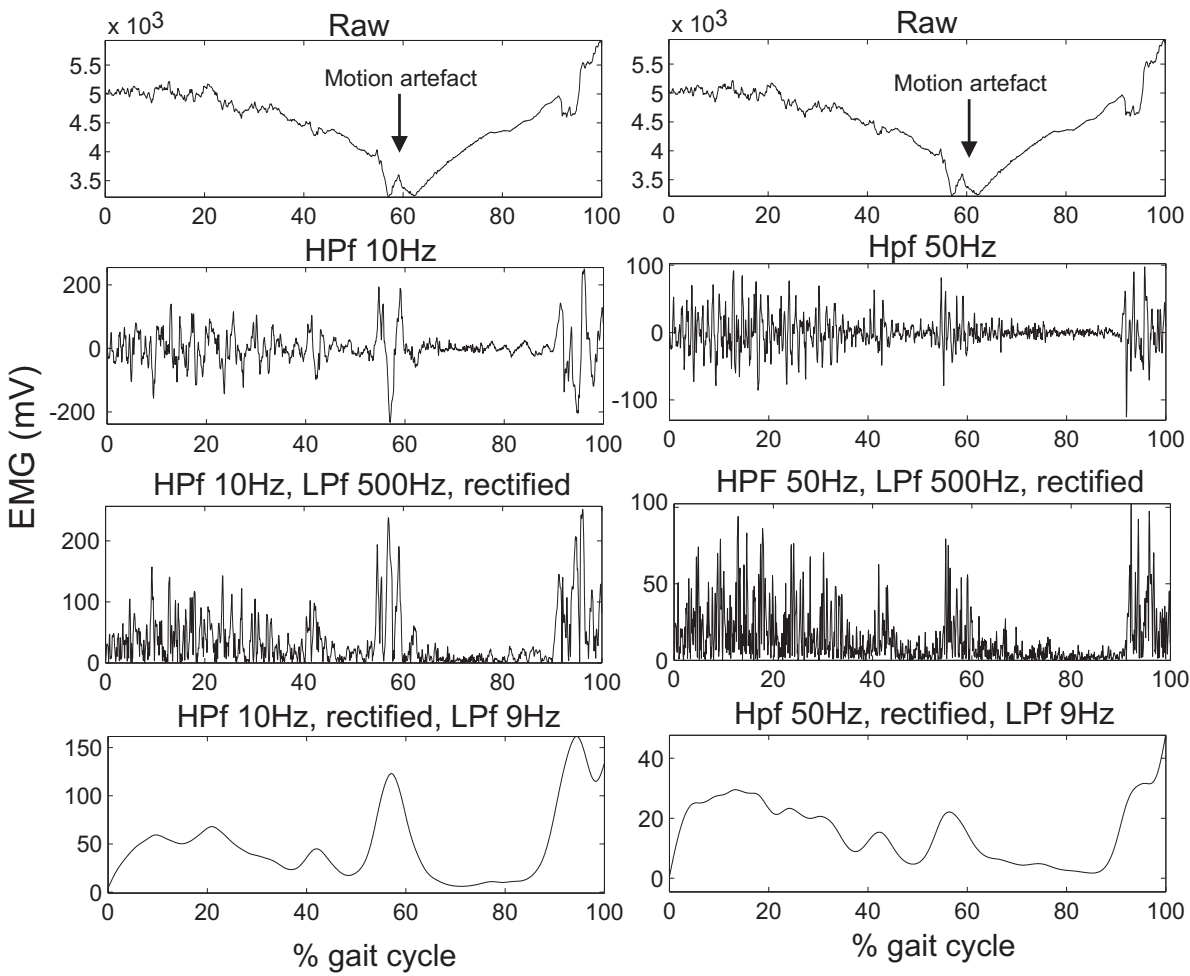

$\mathrm{Hpf} 50 \mathrm{~Hz}$, rectified, LPf $9 \mathrm{~Hz}$

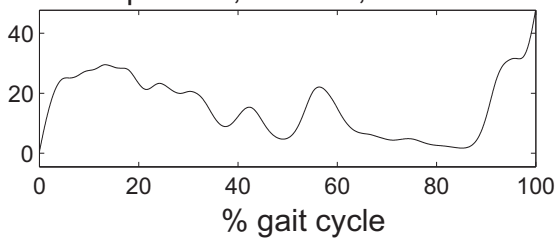

Figure 3.3: An example of one amputee of the biceps femoris EMG with a motion artifact. On the left the data is high-pass filtered at $10 \mathrm{~Hz}$, as in all trials without motion artifacts, but this does not remove the artifacts. On the right the data is high-pass filtered at $50 \mathrm{~Hz}$, which did remove the motion artifact. Trials with this type of artifact that was removed by a $50 \mathrm{~Hz} \mathrm{HP}$ filter, but not by a $10 \mathrm{~Hz} \mathrm{Hp}$ filter were removed from the data. Most trials did not show this type of artifact and therefore the trials with artifacts were removed from the analysis and the original filtering was used. 


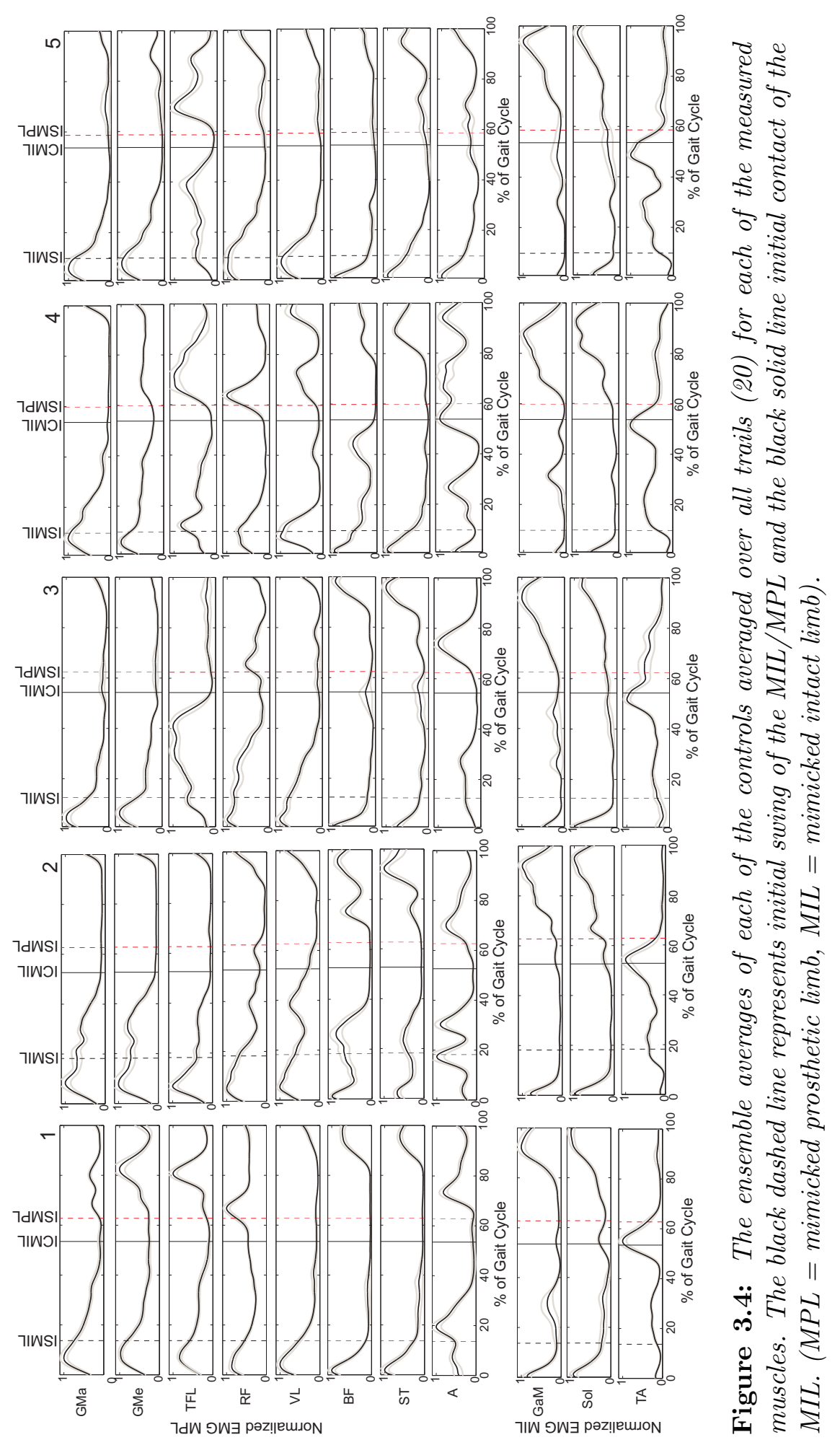



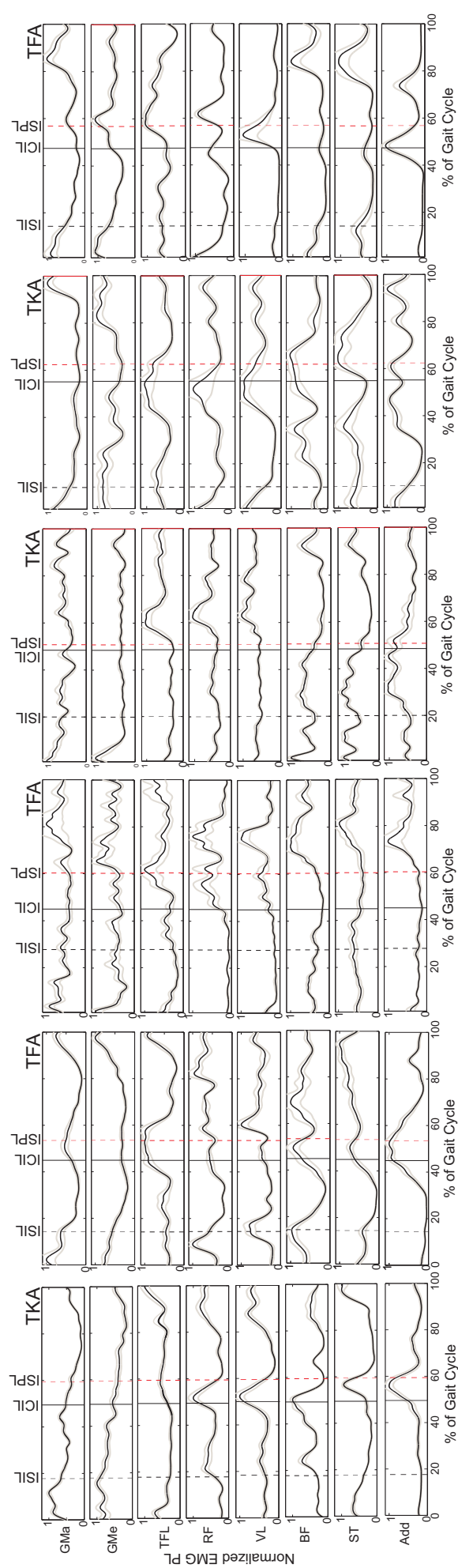
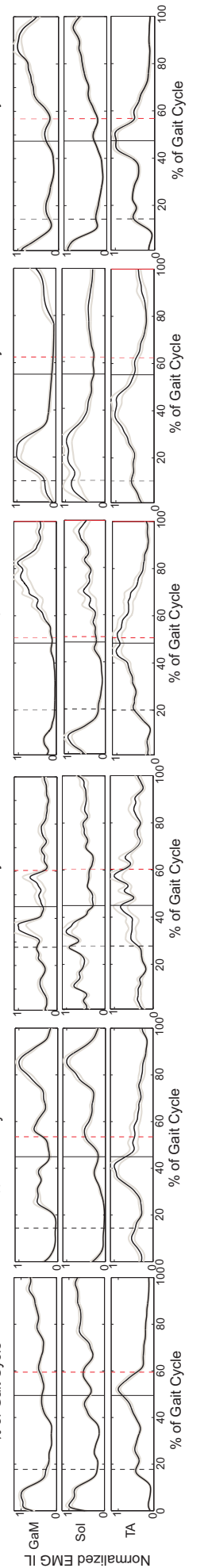

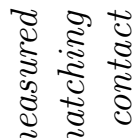

ह ह

竝芯.

० है है

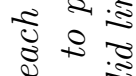

न ने के

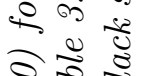

จิ $\approx \frac{0}{0}$

ल) $\frac{\pi}{\pi}$

กิ

$\approx \infty$

$\therefore$ \&

ปิ

ช है ह

웅

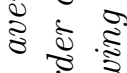

क ริ

晃

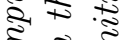

है. . .

荥造

० है है

गิ

व

० 崩

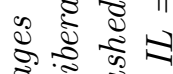

ป ग $\frac{0}{0}$

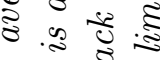

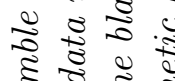

के च ह

के

० है छ़

स है :

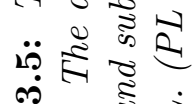

m क ह

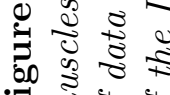

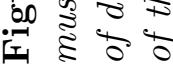




\section{Stance phase}

During the (prosthetic) stance phase, the GMa of the amputee group is active for a longer period after initial contact. All other upper leg muscles are active for a similar or shorter period. In the amputee group, some muscles, become active a second time during stance; the TFL, VL, $\mathrm{BF}, \mathrm{ST}$ and Add. In the controls this second phase of activity during stance for these muscles is not seen. The first period of activity shown for the RF is probably crosstalk by the VL $[109,110]$. The RF becomes active just before terminal stance in controls, but shows no activity in this phase in the amputee group.

During the stance phase of the intact limb, activity of the GaM starts around the same time in controls and amputees, Sol activity of amputees starts a little earlier. The activity of the TiA in amputees continues longer during the stance phase of the intact limb, compared to controls. No significant differences were found between the activation patterns of the stance phase between controls and amputees.

\section{Swing phase}

The differences in muscle activity during the swing phase are larger than for the stance phase. The GMe and GMa of the amputees become active in the second half of the swing phase, whereas in the controls they become active at the end of the swing phase. This increased duration of activation is also seen for the RF, the VL and the BF. The TFL is also active at the transition from stance to swing, and has a later "second" activity onset at the end of the swing phase. The Add is active in amputees before initial swing and at the beginning of the swing phase, which is not the case in controls.

TiA activity during the swing phase of the intact limb starts later in amputees compared to controls. No differences are seen in GaM activity during the swing phase of the intact limb, but the Sol of the amputees shows activity during the first part of the swing phase, where controls do not show this activity. No significant differences were found between the activation patterns of the swing phase of controls and amputees.

\section{Variability}

The overall inter-subject variability of the EMG data of amputees is significantly lower $(\mathrm{p}=0.011)$ than that of controls (figure 3.7). The 


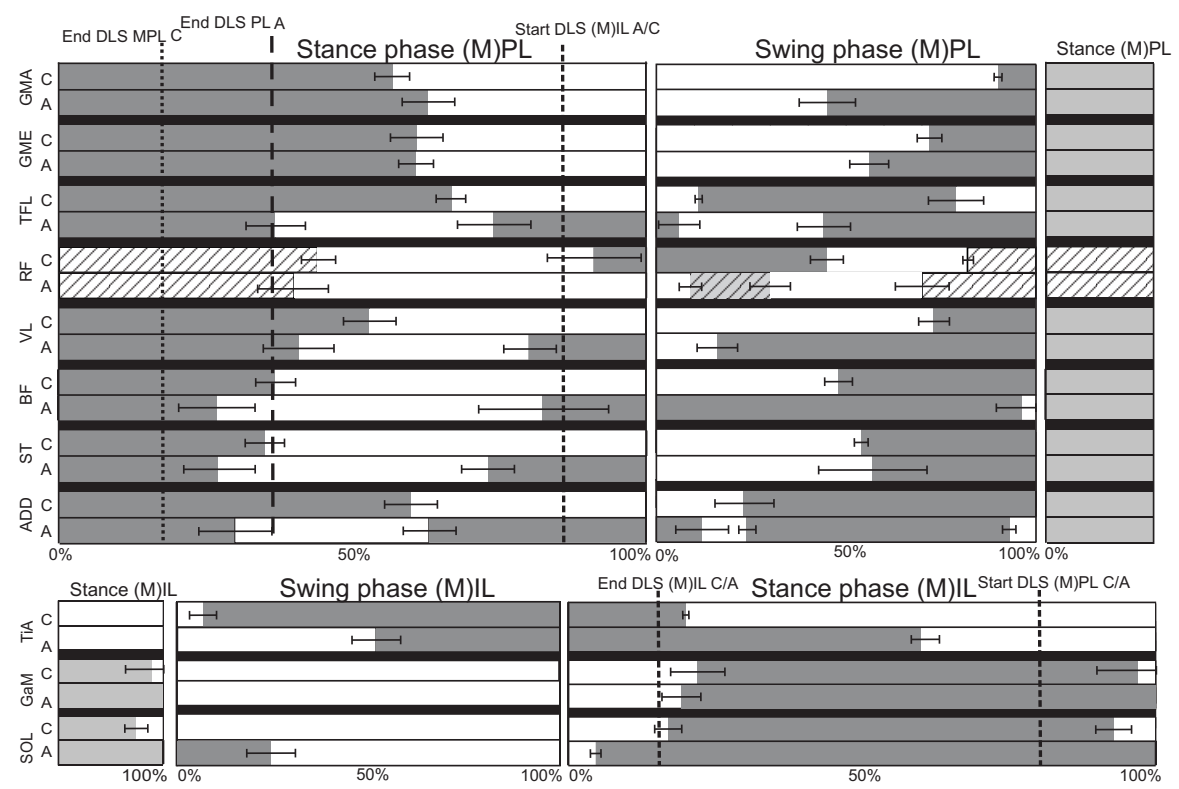

Figure 3.6: The average muscle activity of all muscles, for controls and amputees, as a percentage of the (mimicked) prosthetic stance and swing phase. In dark grey the muscle is "on", in white it is "off" and in hatched white periods of possible cross-talk. The light grey area shows the overlap of the phases. For the stance phase $0 \%$ is IC of the (mimicked) prosthetic limb ((M)PL) and 100\% is initial swing of the (M)PL. The end of the first double stance phase (DLS (M)PL) before (mimicked) prosthetic stance, of controls and amputees are indicated. The start of the second double limb support (DLS (M)IL) is also indicated, which is equal for controls and amputees. For the swing phase, $0 \%$ is initial swing of the (M)PL and 100\% is IC of the (M)PL. The whiskers show the standard error of the mean. All upper leg muscles are measured at the (M)PL and all lower leg muscles at the contralateral (M)IL. Lower leg activity is scaled similarly, but to the phases of the (M)IL. Hereby part of the stance phase (the DLS (M)PL) is placed at the left hand side of the figure.

variability per muscle however is in none of the muscles significantly different. The mean VR of controls ranged from 0.30 to 0.48 , for amputees this range was 0.47 to 0.64 . The SEM of the EMG data is around $3 \%$ of the stance and swing phase for controls, for amputees this is a little higher, around $4 \%$ of the stance and swing phase with some outliers at $12-14 \%$. 




Figure 3.7: The VR of all muscles of controls and amputees. Whiskers give one $S D$.

\subsection{Discussion}

\subsubsection{Kinematic and spatio-temporal data}

The kinematic data showed that the stance phase duration of the intact limb increases and the prosthetic swing phase duration also increases in amputees. This coincides with the general concept that amputees tend to stand longer on their intact limb than on their prosthetic limb, which has also been found in other studies $[24,26]$. Knee flexion during initial stance differs. Controls show a knee flexion of up to $18^{\circ}$, in amputees this is only $4^{\circ}$ even though all amputees had a prosthetic knee which allows knee flexion during stance. This lack of knee flexion might indicate that amputees are not comfortable using knee flexion during initial stance of the prosthetic limb, which may be caused by a lack of trust or experience in using the MPC knee to the full potential. Hip adduction and abduction are also reduced in amputees, which was also reported by Jaegers et al. [26]. The reduction in hip adduction has most likely only a small effect on the walking pattern, as it is only a few degrees less than in controls. During normal single limb stance a small amount of adduction is seen, to ensure that the center of mass does not have to move laterally to keep it above the supporting surface. However when amputees are in prosthetic single limb support they will not bring their COM above their support surface, but keep it more medially. This can be explained by the fact that in the frontal plane they have little opportunity to correct themselves, too much lateral motion will cause a fall. This reduces the need for adduction in stance. The reduced abduction may change the walking pattern of amputees. Hip abduction 
is used to "shorten" the leg to ease foot clearance during the transition from the stance to the swing phase. However TFA generally find it more difficult to perform hip abduction, which makes foot clearance more difficult. The reduction in hip abduction may create the need for more adaptations from the intact limb, for instance increased plantar flexion during single intact limb support (vaulting).

\subsubsection{EMG}

The differences found in muscle activity between prothetic users and controls are mainly present in the (pre)swing phase. Muscle activity of controls resembles that of previous studies, although muscles show activity for a longer period of time $[21,22]$. This may be due to the onset detection method, but the exact methods used in the previous studies were not described. Therefore it is hard to find a clear explanation for this discrepancy.Visual comparison of raw and filtered EMG data showed comparable EMG quality between controls and amputees.

\section{Stance phase}

When the gait stance and swing phases are compared separately, muscles in amputees do not seem to be active for much longer than in controls. At the end of the stance phase a period of activity is seen in most of the upper leg muscles, starting around the beginning of the second double support phase. This may be the mechanism by amputees to increase socket fitting at the end of the stance phase, to prepare for lifting of the prosthesis in the swing phase [38].

Lower leg muscles of the contralateral side show a prolonged activity during stance. This increased activity could be used to ease foot clearance, ankle plantar flexion of the intact limb is used to virtually lengthen the intact limb. The prolonged activity can also be explained by the increased push-off needed from the intact limb, to propel the body forwards, to compensate for the lack of push-off on the prosthetic side. This coincides with the kinetic data, which showed increased work at the hip and plantar flexors of the intact limb $[3,95,96]$.

\section{Swing phase}

Some of the upper leg muscles of the amputees, the BF and the VL, remain active for almost the complete swing phase. The other muscles all become active again at the end of the swing phase to prepare for initial contact. These muscles show an earlier activity onset than in controls, 
which may be explained by the walking strategy of amputees. Many amputees try to fully extend the knee to ensure it is locked at the end of the swing phase which is also confirmed by the reduced knee flexion during initial stance.

Our results resemble the results presented by Jaegers et al. [27], as far as they can be compared. They only showed muscle activity for the complete gait cycle and no exact onset timings were calculated. They also reported activity before initial swing and found differences between subjects with an amputation in the proximal or distal half of the upper leg [27]. In the current study all amputees were amputated at the distal half of the upper leg.

In some muscles the activity is slightly longer or shorter compared to Jaegers et al. [27]. This can be due to the separated stance and swing phases in the current study and due to different approaches in detection times.

TFA showed a different activation pattern in some phases of the gait cycle, which shows that they adapt to their new prosthetic situation. Although the results show that consistent muscle activity can be measured inside the socket of TFA, the usability for prosthetic control is questionable. Variability between the amputees is higher, but patterns within the amputees are consistent. Although muscle activity patterns can change due to the disturbed anatomy by the amputation and by use of the prothesis, training may allow TFA to learn new walking patterns which in turn may need adaptations in the muscle activity patterns to control a prosthesis [99].

\section{Variability}

The overall inter-subject variability of the EMG data from the amputees was significantly higher than that from controls. VRs per muscle were however for none of the muscles significantly different. Granata et al. [111] reported VRs in healthy adults between 0.17 and 0.27 , although they can go up to 0.76 in healthy adults [112]. The main reason for a higher VR in amputees (up to 0.64) is most likely the lower walking speed [80].

Many of the muscles in the upper leg of the amputees are cleaved. The electrodes were placed and tested for activity according to the SENIAM standards. However, due to the amputation some muscles may have a different location and the location of the electrodes may not 
have been ideal. Rotations in the socket may also affect the position of the electrodes with respect to the muscle. Poor socket fitting will affect the repeatability of the signal, this will induce more noise and the prosthetic user may show more muscle activity to properly control or fit the prosthesis. One subject complained of non-optimal socket fitting as it was too large. This subject had a higher VR. None of the subjects complained about the EMG electrodes, they did not seem to effect the socket fitting. However, this does not explain the increased VR in the lower leg muscles of the contralateral limb and the hip muscles. This might indicate that the walking pattern of amputees is less consistent than that of controls. The standard deviations within amputees for spatio-temporal and kinematic data were also larger than in controls.

\section{Methodological considerations}

The amputees were a mixed group. No inclusion criteria for type of amputation or time since amputation were added. The average age (52.3) was larger than that of the controls (23). Previous studies have shown that aging may affect the spinal cord activity, walking speed and cause a higher spread in muscle activation $[21,113]$. Also three prosthetic users were only prosthetic users for 5-6 month, of whom the EMG pattern may still change over time. Two of them were the elderly subjects with vascular diseases. One of these subjects also walked with a walking aid, which may also effect the muscle activity [114], both subjects had higher VRs. Nevertheless, even with the large variability in the group, no large deviations were seen in the EMG patterns of these subjects.

A more homogeneous and larger group of amputees with similar prosthesis may reduce the variability between the subjects. We did not find any obvious differences between the different knees, but this may also be caused by the low number of amputees. Including amputees with a short residual limb as Jaegers et al. [26,27] did, can be an interesting addition.

Measurements were performed inside the socket of the amputees. The residual limb-socket interface may have lead to increased motion artifacts, compared to using an experimental socket with build in EMG sensors. Data were checked for these artifacts. It occurred only occasionally during initial contact or initial swing that these artifacts were not removed by filtering. Trials with motion artifacts were removed, but this still allowed at least 20 steps to be included per subject. No motion artifacts were found in controls. Although we measured EMG inside the socket with reasonable quality, we did not test the reliability and validity compared to EMG measured using an experimental socket. 
We placed electrodes and performed EMG measurements according to the SENIAM standards, which are based on normal anatomy. No information on actual muscle locations were available, for instance from MRI. After electrode placement muscle activity was checked using selective muscle contraction. Only occasionally electrodes needed to be replaced, for a better location with respect to the muscle belly, but never more than 2-4 cm from the original placement. Therefore normal anatomy was assumed in amputees, with respect to cross-talk.

Surface EMG was used for ease of electrode placement and comfort to the patient. Intramuscular EMG may have given less cross-talk and possibly more information on specific muscle activity, but it is impossible to use in the own socket of the amputees and very uncomfortable to the patient.

\subsection{Conclusion}

In amputees the double support phase before the prosthetic stance phase increases significantly and the prosthetic swing phase shortens. EMG patterns mainly differ at the end of the stance phase and in the swing phase. These changes can explain the changes in walking strategy, but are likely also required to improve socket fitting. In this study EMG was measured inside the socket of amputees and the data showed to be of comparable quality to that of controls. Variance within each amputee is higher than in controls, but variability in the kinematic data between the amputees is also higher. The increased variance may mainly be caused by the variability in walking pattern and cleavage of muscles. 


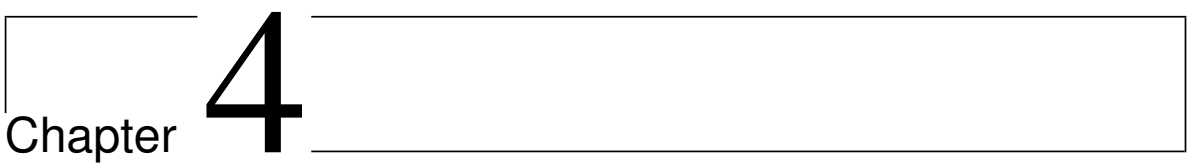

\section{EMG of transfemoral amputees and controls during slope and stair walking ${ }^{1}$}

The purpose of this study was to compare gait patterns of amputees during slope and step-by-step stair walking to those of non-amputees.

Spatio-temporal, kinematic and electromyographic data obtained from the prosthetic upper leg of six transfemoral amputees, was compared to that of five controls. On-off timings for stance and swing phase were determined for slope and stair walking (up and down) as well as relative gait phase durations and joint angles.

In slope walking the double support phase of the prosthetic leg is twice as long as in controls. Prolonged and additional muscle activation is seen in amputees mainly in the stance to swing transition for slope ascent and descent. In stair walking the stance phase of the intact leg increases ( $70 \%$ ), the double support phase also increases in stair ascent (18\%) but decreases (6\%) in stair descent. Hip abduction was increased in order to achieve foot clearance under both conditions. Prosthetic knee flexion in stance for slope and stair walking is absent. In stair ascent the glutei muscles were more active in stance, in swing upper leg muscles showed more activity. In stair descent the hip extensors showed more activity in the swing to stance transition. All changes are likely to compensate for reduced progression over the foot, the lack of actuation of the prosthetic knee and ankle, and to increase socket fitting. The intact leg is used more for stability and support.

\footnotetext{
${ }^{1}$ Submitted for publication
} 


\subsection{Introduction}

Upper leg prostheses do not fully replace the functionality of the lower limb, therefore transfemoral amputees (TFA) need to adapt their walking pattern. One of the adaptations is an increased support phase of the intact limb $[3,24,26]$.

Although slope and stair walking are both important in daily life activities, biomechanical research in TFA has largely focussed on level walking $[23,24,26,115]$. Therefore limited information is available on the adaptation of TFA during slope and stair walking [116]. Differences in gait pattern between level, stair and slope walking can be found in kinematic and kinetic variables and electromyographic (EMG) data.

For slope ascent and descent in amputees similar kinematic changes are seen when compared to level walking [23,117], but little information is available on muscle activity in amputee slope walking. In addition, few studies have looked at stair ascent and descent in TFA, and mostly at step-over-step (SOS) [41,118-121]. During rehabilitation, most amputees learn to ascent and descent stairs step-by-step (SBS) [2].

\section{Slope ascent}

In slope ascent, non-amputees show less knee extension during initial contact (IC) compared to level walking [122-124]. During this phase the internal ankle plantar flexor moment, the knee flexor moment and the hip extensor moment are also increased [122-124]. During mid-stance an increase in knee extension moment is seen. In addition, the amplitude and duration of the biceps femoris, semimembranosis, rectus femoris and vastus medialis are increased $[124,125]$. In terminal-stance an increase in plantar flexion is seen. This results in a virtual lengthening of the leg, thereby contributing to the foot clearance of the contralateral leg. Push-off can increase with 50\% compared to level walking [122], which is reflected in an increased magnitude and duration of gastrocnemius activity [125]. The increased ankle push-off contributes to the necessary vertical displacement of the center of mass and propels the leg into swing. Tibialis anterior activity increases during swing.

The gait pattern of amputees during slope ascent changes when compared to level walking. Wolf et al (2012) showed that the prosthetic stance phase $(60 \%)$ is reduced compared to the stance phase of the intact limb (70\%) [117]. In addition, several changes in kinematics are seen [116]. The prosthetic knee shows decreased knee flexion during the 
swing phase. In the intact leg an increased knee flexion is seen during the loading response. In addition, in terminal stance a reduction of maximal hip extension is found. Finally, an increase in ankle plantar flexion is seen during pre-swing.

When kinematics of slope ascent of amputees is compared to non-amputees, several differences are found [116]. The hip and knee of the amputated leg show decreased flexion in stance and swing when compared to nonamputees. In addition, the intact ankle shows a decrease in dorsal flexion during the stance phase and an increase in plantar flexion during the swing phase compared to non-amputees.

\section{Slope descent}

Slope descent in non-amputees shows similar adaptations to slope ascent. Wolf et al. [117] found that in slope descent the prosthetic stance phase is also shorter (57\%) compared to the stance phase of the intact leg (70\%) using a C-leg (Otto Bock). Compared to level walking hip flexion at IC is reduced, whereas knee flexion is increased. The ankle dorsiflexion moment and amplitude of tibialis anterior activity are increased in this phase. In early to mid-stance the hip and knee flexion and ankle dorsiflexion are increased. The hip flexion and ankle dorsiflexion moments are decreased during this phase, but the knee shows a flexion moment rather than a extension moment [122,125-127]. At mid-stance the hip flexion moment changes to a hip extension moment, which is earlier than in level walking. The rectus femoris and the vastus medialis show a higher amplitude and a longer duration with an earlier onset. Both soleus and gastrocnemius activity show a decrease in amplitude, an earlier onset and a longer duration $[125,127]$. At the end of the stance phase the hip shows reduced extension and the ankle shows more dorsiflexion [127]. During the swing phase the main differences are seen in the joint angles. There is less hip flexion, reduced ankle plantar flexion and increased knee flexion compared to level walking [122, 126, 127].

In amputees the prosthetic leg shows a decrease in hip and knee flexion during the swing phase, compared to level walking and compared to non-amputees. At the start of the swing phase the ankle shows dorsal flexion rather than plantar flexion as is the case in non-amputees [23]. At the intact side the hip joint angles during the entire gait cycle are similar to level walking and to non-amputees. A decrease in knee flexion during late stance and the swing phase is seen, compared to non-amputees. Compared to level walking however the intact knee shows more flexion in this phase. At the ankle no changes were found [23]. 


\section{Stair Ascent}

Research in non-amputee SBS stair ascent showed that the leading limb shows similar knee flexion moments and angles as in SOS stair ascent during the entire step [128]. The trailing limb on the other hand shows lower knee flexion angles in swing and stance. The knee flexion moment at initial contact is less than half of that of the leading limb. In the frontal plane however differences are only seen in the larger adduction angle in swing of the leading limb compared to the trailing limb.

Only few studies have looked at stair ascent in TFA, and mostly at SOS [41,118-121]. During rehabilitation, most amputees learn to ascent stairs SBS (see figure 4.1) [2].

For amputees, the prosthetic limb would be the trailing limb in SBS stair ascent, the intact limb is used to raise the body (see also figure 4.1). [128] Bae et al. [98] found in TFA that at the intact side the knee extensor muscles showed higher activity in SBS stair ascent. They also compared SOS stair ascent to SBS stair ascent and level walking and found that the loads on knee and hip at the intact leg increased in SOS stair ascent [98].

\section{Stair descent}

SBS stair descent in non-amputees (see also figure 4.1) the trailing leg showed similar moments, forces and angles as in SOS stair walking during the entire step. For the leading leg, the knee flexion angle during swing and the knee flexion moment during stance are considerably less. During the entire stance phase of the leading limb no power generation nor absorption peak at the knee is seen. [128]

The main differences in stair descent between non-amputees and TFA are caused by the lack of active ankle plantar flexion, knee flexion and extension at the prosthetic side. SOS descent is only possible using prosthetic knee joints with flexion resistance or by using the handle bars [119]. TFA start their SBS stair descent with the prosthetic leg as the leading limb (see also figure 4.1). Jones et al. [129] investigated initial contact of one step descent in TFA using non-actuated prosthesis. They found that the ratio of peak force (longitudinal to the limb) to the maximal change in limb length, normalized to body mass was increased in the prosthetic limb of TFA compared to controls. They also found that TFA landed on an almost straight knee $\left(2^{\circ}\right)$ and that the ankle angle at foot contact is dependent on the prosthetic alignment. The land- 
ing on a straightened and near vertical limb was an expected strategy from amputees to place the center of mass directly above and anterior of the knee to prevent a knee collapse. [128] Bae et al. [98] found that the intact limb of TFA showed a higher level of activity during stance in the rectus femoris, vastus lateralis and vastus medialis, but lower in the semitendinosis, the gluteus maximus, the biceps femoris, the soleus, gastrocnemius medialis and the tibialis anterior.

We aim to provide more insight into the adaptations of the residual leg of a person with an amputation during slope and stair walking. Therefore we collected kinematic and EMG data of TFA during slope walking and step-by-step stair negotiation. The main goal was to determine the muscle activation patterns and compare these to data of control subjects.

\subsection{Methods}

Methods and data analysis were similar to those described in chapter 3. The information on participants (table 3.1), measurement setup and data analysis of kinematic data and EMG recordings can be found in sections 3.2.1, 3.2.2, 3.2.4 and 3.2.4 respectively. The muscles measured were: m. gluteus maximus (GMa), m. gluteus medius (GMe), m. tensor fasciae latae (TFL), m. rectus femorus $(\mathrm{RF}), \mathrm{m}$. vastus lateralis (VL), $\mathrm{m}$. biceps femoris $(\mathrm{BF}), \mathrm{m}$. semitendinosis $(\mathrm{ST}), \mathrm{m}$. adductor magnus $(\mathrm{ADD})$ at the prosthetic limb and at the contralateral lower limb: $\mathrm{m}$. tibialis anterior (TA), m. gastrocnemius medialis (GaM) and the $\mathrm{m}$. soleus (Sol).

\subsubsection{Procedures}

For both experiments the subjects were asked to walk at a self selected speed. After data recording was started, the sync was pressed and subjects started ascending the slope/stairs. At the top of the slope/stairs they were asked to stop, turn around, wait 2-3 seconds, press the sync and descent the slope/stairs; this constituted one trial. Four slope trials were performed in all subjects. For the stair experiments, nine subjects performed eight trials, two amputees only performed four trials (at least 12 steps per person). Controls and amputees were measured at two different locations. For the experiments in controls we used a $7^{\circ}$ slope of 7 meters and a set of 5 stairs each $20 \mathrm{~cm}$ high and 32 deep, for amputees 
we used a $10^{\circ}$ slope of 5 meters long and a set of 3 stairs, each $19 \mathrm{~cm}$ high and 33 deep.

\subsubsection{Data analysis}

From the footswitch data the timings of initial contact (IC), terminal stance, initial swing (IS) and loading response of each leg were determined. All steps per subject were aligned at IC of the prosthetic leg. For slope walking, steps were cut from the EMG and inertial sensor data, from IC to IC of the prosthetic leg.

For stair ascent one step starts at IS of the intact leg and ends at IC of the prosthetic leg.(figure 4.1) For stair descent, a step starts at IS of the prosthetic leg and ends at IC of the intact leg. For all steps IS was defined as the moment when both footswitches of one foot, lose contact with the floor and IC as the moment when one of the switches showed contact again.

\subsection{Results}

All comparisons presented here will be between the prothetic leg of the amputees and the mimicked prosthetic leg of the controls, unless stated otherwise.

\subsubsection{Spatio-temporal data}

- Slope - The walking speed of transfemoral amputees during slope ascent and descent was significantly lower compared to controls. In slope ascent the stance phase of the intact limb and the swing phase of the prosthetic limb are significantly increased in amputees. The swing phase of the intact limb and the stance phase of the prosthetic limb are significantly decreased. For slope descent the same changes in gait phases are seen, although the stance and swing phase of the prosthetic limb of controls and amputees are not significantly different (table 4.2). Phases of slope ascent and descent can be seen in figure 4.2.

- Stair - The horizontal velocity of stair ascent and descent is lower in amputees than in controls. In stair ascent, the prosthetic stance phase, the swing phase of the intact limb and the double support phase are significantly shorter, but the stance phase of the intact leg is significantly longer in amputees compared to controls. In stair descent of the amputees, the stance phase of the intact limb and the double support phase 

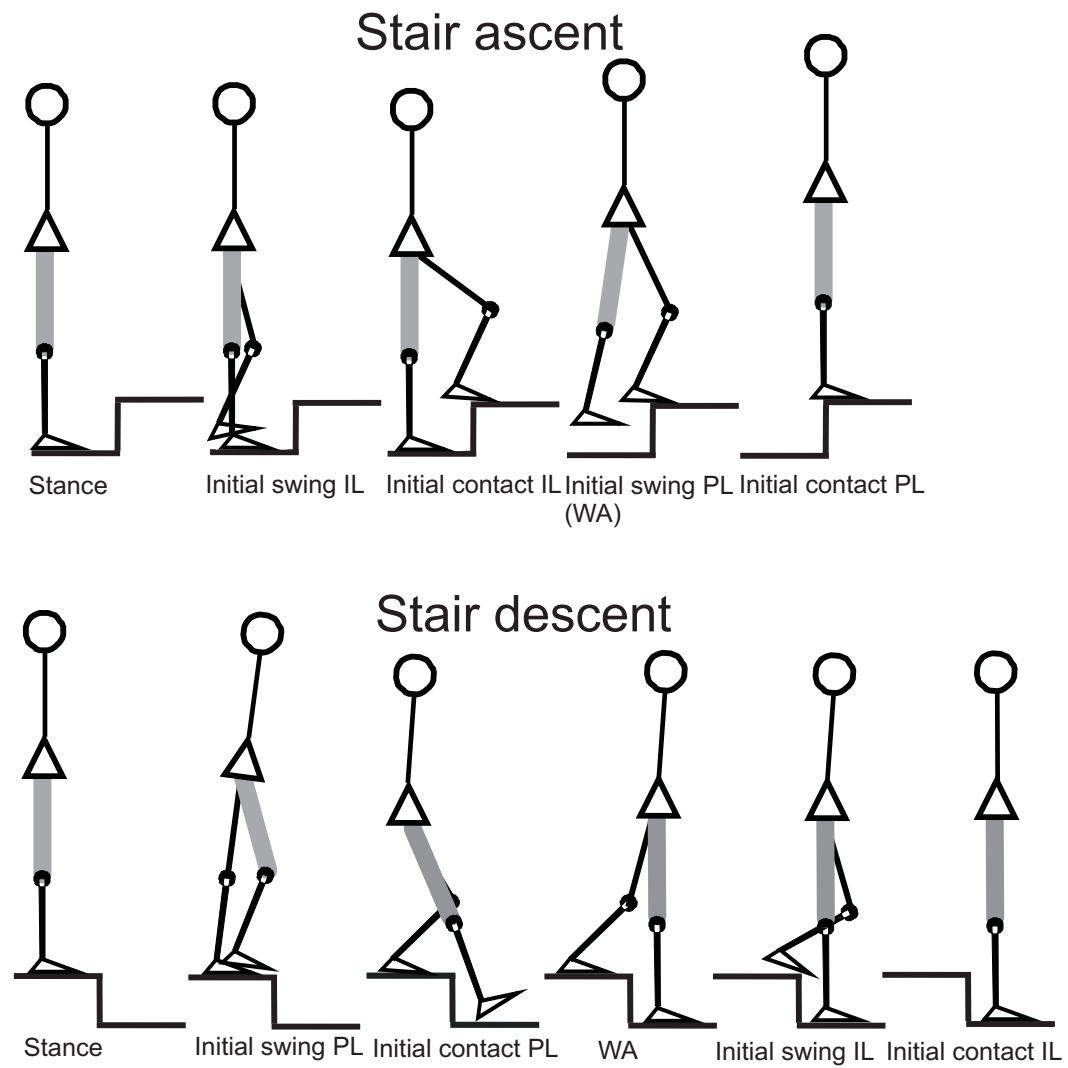

Figure 4.1: Schematic drawing of stair ascent and descent for amputees. The thick grey line is the prosthetic side, the thin black line the intact side.

are significantly increased compared to controls. (table 4.2) Phases of slope ascent and descent can be seen in figure 4.3. 
Table 4.1: Gait phases and maximal joint rotations in slope walking

\begin{tabular}{|c|c|c|c|c|}
\hline Activity & \multicolumn{2}{|c|}{ Slope ascent } & \multicolumn{2}{|c|}{ Slope descent } \\
\hline Phase & $\mathrm{CS}$ & AS & $\mathrm{CS}$ & $\mathrm{AS})$ \\
\hline & value $(\mathrm{SD})$ & value(SD) & value $(\mathrm{SD})$ & value $(\mathrm{SD})$ \\
\hline Speed $(\mathrm{m} / \mathrm{s})$ & $0.92(0.1)$ & $0.55(0.2)^{a}$ & $0.91(0.1)$ & $0.47(0.2)^{a}$ \\
\hline St PL $(\%)$ & $59(1)$ & $53(7)^{a}$ & $59 \%(2)$ & $52(8)$ \\
\hline St IL $(\%)$ & $59(2)$ & $74(9)^{a}$ & $58 \%(2)$ & $74(12)^{a}$ \\
\hline Swing PL(\%) & $41(1)$ & $47(7)^{a}$ & $41 \%(2)$ & $48(8)$ \\
\hline Swing IL(\%) & $41(1)$ & $26(9)^{a}$ & $42 \%(1)$ & $23(7)^{a}$ \\
\hline DLS PL(\%) & $10(1)$ & $20(10)$ & $8 \%(1)$ & $20(6)^{a}$ \\
\hline DLS IL $(\%)$ & $9(2)$ & $9(3)$ & $8 \%(2)$ & $10(1)$ \\
\hline Max hip flex $\left(^{\circ}\right)$ & $46(12)$ & $32(6)$ & $35(6)$ & $23(6)$ \\
\hline Max hip ext $\left(^{\circ}\right)$ & $2(2)$ & $9(6)$ & $3(2)$ & $9(8)$ \\
\hline Max hip add $\left(^{\circ}\right)$ & $8(8)$ & $7(5)$ & $7(7)$ & $7(5)$ \\
\hline Max hip abd $\left(^{\circ}\right)$ & $10(6)$ & $3(3)^{a}$ & $10(7)$ & $5(5)$ \\
\hline Max knee flex st $\left(^{\circ}\right)$ & $14(5)$ & $3(3)^{a}$ & $13(5)$ & $2(1)^{a}$ \\
\hline Max knee flex sw $\left({ }^{\circ}\right)$ & $42(7)$ & $42(16)$ & $53(7)$ & $43(9)$ \\
\hline
\end{tabular}

(Stat $=$ statistical note, IL $=$ intact leg, $\mathrm{PL}=$ prosthetic leg, SLS $=$ single limb support, DLS $=$ double limb support. a = significant difference in slope walking with $\mathrm{P}<0.05$ ) 
Table 4.2: Gait phases and maximal joint rotations in stair walking

\begin{tabular}{|l|llll|}
\hline Activity & \multicolumn{2}{|c|}{ Stair ascent } & \multicolumn{2}{c|}{ Stair descent } \\
\hline Phase & CS & AS & CS & AS \\
\hline & value(SD) & value(SD) & value(SD) & value(SD) \\
\hline Speed (m/s) & $0.19(0.0)$ & $0.16(0.1)$ & $0.21(0.0)$ & $0.15(0.1)$ \\
St PL(\%) & $56(2)$ & $40(7)^{b}$ & $54(2)$ & $48(15)$ \\
St IL(\%) & $55(4)$ & $67(6)^{b}$ & $56(2)$ & $70(7)^{b}$ \\
Swing PL(\%) & $44(2)$ & $61(7)$ & $46(2)$ & $52(15)$ \\
Swing IL(\%) & $45(4)$ & $33(6)^{b}$ & $44(2)$ & $30(7)$ \\
DLS PL(\%) & $11(2)$ & $6(5)^{b}$ & $11(4)$ & $18(13)^{b}$ \\
\hline Max hip flexion $\left(^{\circ}\right)$ & $10(4)$ & $10(6)$ & $23(2)$ & $15(9)$ \\
Max hip extension $\left(^{\circ}\right)$ & $8(2)$ & $2(1)^{b}$ & $2(1)$ & $2(1)$ \\
Max hip adduction $\left(^{\circ}\right)$ & $2(1)$ & $1(0)$ & $3(2)$ & $2(2)$ \\
Max hip abduction $\left(^{\circ}\right)$ & $6(4)$ & $13(7)$ & $4(4)$ & $10(5)$ \\
Max knee flex stance $\left(^{\circ}\right)$ & $9(2)$ & $0(0)^{b}$ & $5(4)$ & $1(3)$ \\
Max knee flex swing $\left(^{\circ}\right)$ & $34(5)$ & $4(2)^{b}$ & $33(3)$ & $14(15)$ \\
\hline \hline
\end{tabular}

(Stat $=$ statistical note, IL $=$ intact leg, $\mathrm{PL}=$ prosthetic leg, SLS $=$ single limb support, DLS $=$ double limb support. $\mathrm{b}=$ significant difference in stair walking with $\mathrm{P}<0.05$ )

\section{Joint rotations}

- Slope - Knee flexion during stance in slope ascent and descent is significantly reduced in amputees compared to controls (table 4.2). Maximal hip abduction in swing for slope ascent is significantly decreased compared to controls. Three subjects showed adduction in the swing phase in slope ascent, three others did not. Maximal hip flexion in late stance in slope ascent and descent, is also reduced in amputees, but not significantly. Examples of angular rotations of hip, knee and ankle during slope ascent and descent can be found in figure 4.4.

- Stair - In stair ascent amputees show significantly reduced hip extension in stance and swing on the prosthetic side (table 4.2). Hip abduction, in stair ascent, is increased compared to controls, but not significantly. Almost no knee flexion is visible in stair ascent in amputees.

In stair descent in amputees, hip flexion is reduced and hip abduction is increased compared to controls, but not significantly. Knee flexion in swing is larger in controls than in amputees. This is not significant, 

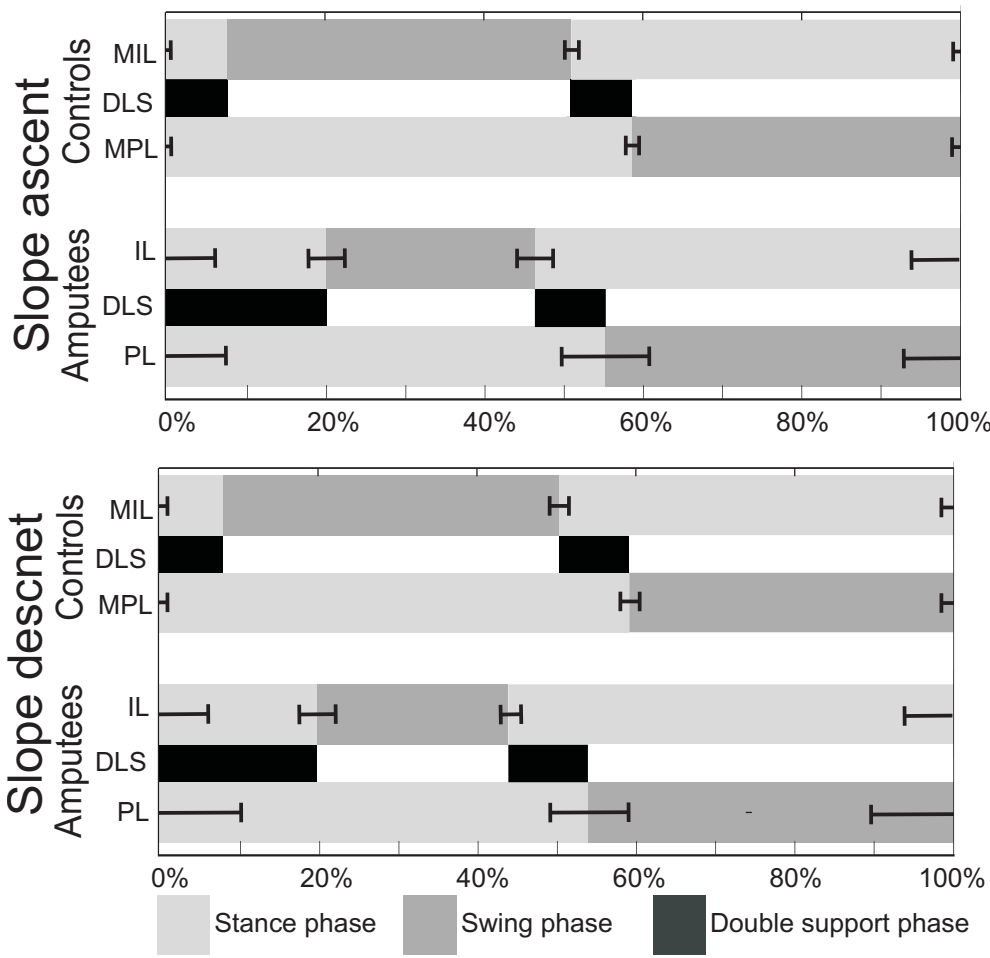

Figure 4.2: Gait phases for controls and amputees, as percentages of one full stride for slope ascent (top) and descent (bottom). In dark grey the swing phase, in light grey the stance phase and in black the double support phases. (M)IL = mimicked intact limb, (M)PL = mimicked prothetic limb, $D L S=$ double limb support. The whiskers give one $S D$.

but 4 amputees had an average knee flexion angle in swing of $4^{\circ}$ and for the other two it was $32^{\circ}$ on average. Examples on angular rotations of hip, knee and angle can be found in figure 4.5. Examples of angular rotations of hip, knee and ankle during stair ascent and descent can be found in figure 4.5 .

\subsubsection{EMG data}

\section{Slope}

- Slope ascent - In amputees during the stance phase the duration of muscle activity of the upper leg muscles is generally longer than in controls (figure 4.6). This is especially the case for the GMa, the BF and the ST. In amputees the activity of the GMe, the RF, the VL, the ST 

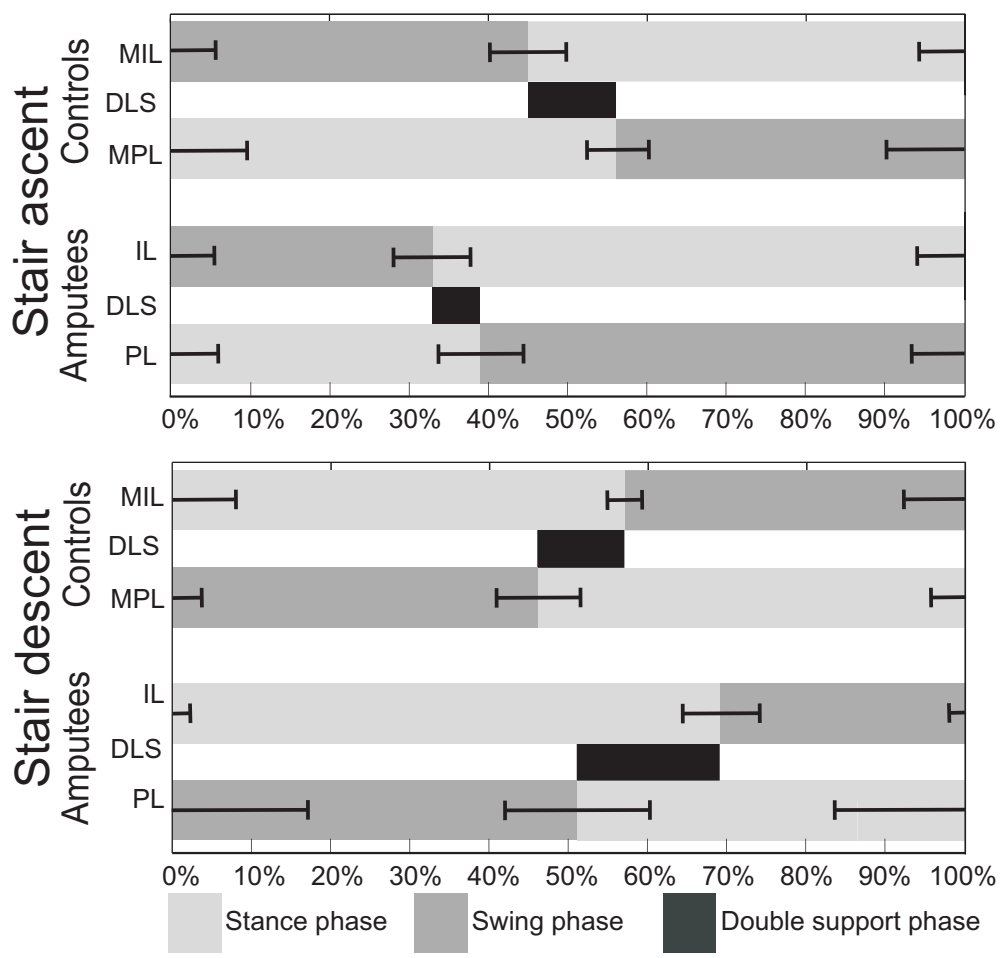

Figure 4.3: Phases in stair walking step-by-step for controls and amputees, as percentages of one full step in stair ascent (top) and stair descent (bottom). In dark grey the swing phase, in light grey the stance phase and in black the double support phases. (M)IL = (mimicked) intact limb, (M)PL = (mimicked) prosthetic limb, DLS = double limb support. The whiskers give one SD.

and the Add starts again just before the swing phase. In controls this is only seen in the GMe and the RF. For the contralateral lower leg muscles no obvious changes are seen in the stance phase.

During the swing phase prolonged activity is seen in amputees, compared to controls. In all muscles of the amputees a period of activity is seen around the stance to swing transition. Either starting just before the swing phase and continuing during the swing phase or starting at initial swing. RF and the ST activity is continued until the end of the swing phase. For the other muscles, this activity ends around $50 \%$ of the swing phase. At the end of the swing phase all muscles, except the ADD, show another burst of activity, starting generally earlier than in control subjects. 


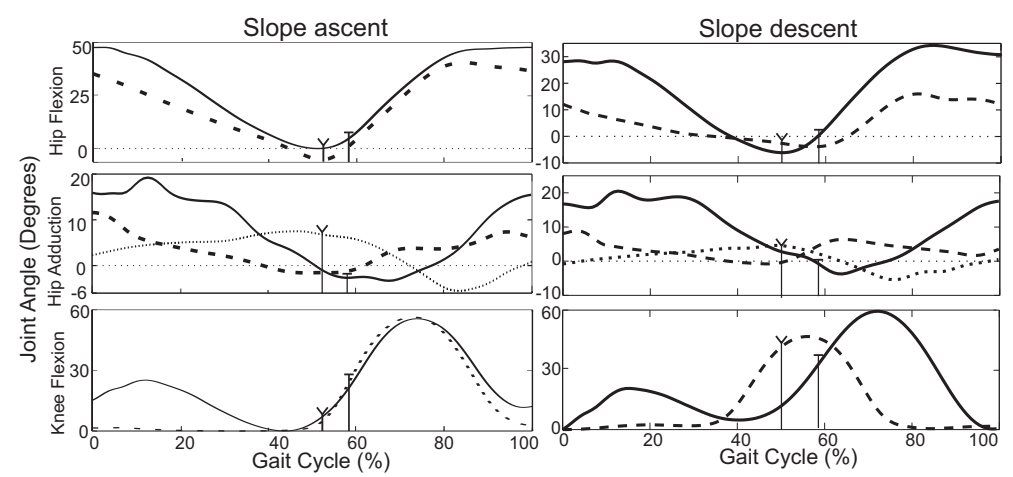

Figure 4.4: Joint angles of the prosthetic side of one representative control subject (-) and one representative amputee 1 (- - -), in slope walking. Except for hip ab/adduction where in the amputee group two main patterns were visible, without and with circumduction. Amputee 1 is representative for half the group, without circumduction, and amputee $2(\cdots)$ for the other half. For hip flexion and knee flexion, amputee 1 shows a representative pattern for the whole group. Initial swing is indicated by vertical line $(Y)$ for amputees and $(\top)$ for controls.

- Slope descent - In both subject groups all upper leg muscles are active, with similar duration, during the first part of the stance phase (figure 4.7). The VL shows a shorter period of activity in amputees and the ADD only shows a short burst at the beginning of the stance phase. In amputees all muscles, except the GMa, show a period of activity in the stance to swing transition. This activity is also seen in the RF, the $\mathrm{VL}$ and the ST of the controls. In the contralateral lower leg, GaM activity in amputees starts earlier at the end of the stance phase.

The ST is active for almost the entire swing phase in both groups. In controls all other muscles starting their activity just before the start of the swing phase, end their activity before $50 \%$ of the swing phase. In amputees the VL and the RF show activity during almost the complete swing phase. In both groups all muscles, except the TFL and the ADD in amputees, become active again at the end of the swing phase, in amputees a little earlier than in controls. The TiA of the amputees shows a longer period of activity during the swing phase. 


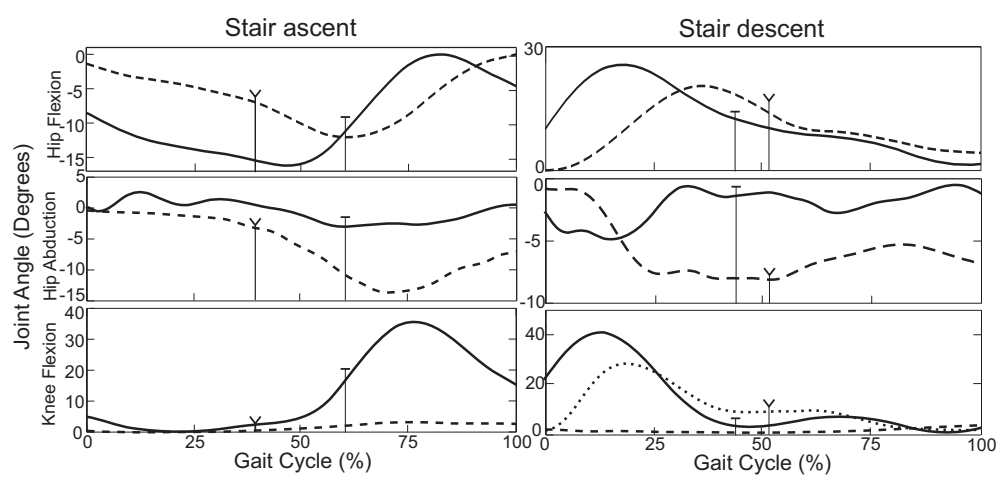

Figure 4.5: Joint angles of the prosthetic side of one representative control subject (-) and one representative amputee 1 (- - -), in stair walking. Except for knee flexion/extension for stair decent, where in the amputee group two main patterns were visible, without and with knee flexion. Here amputee 1 is representative for 4 of the 6 amputees, without knee flexion, and amputee 2 (...) for the other two. For hip flexion and ad/abduction and knee flexion in stair ascent, amputee 1 shows a representative pattern for the whole group. Initial swing is indicated by vertical line $(Y)$ for amputees and $(\top)$ for controls. Hip flexion, hip adduction and knee flexion are represented by positive angles. 


\section{Slope ascent}
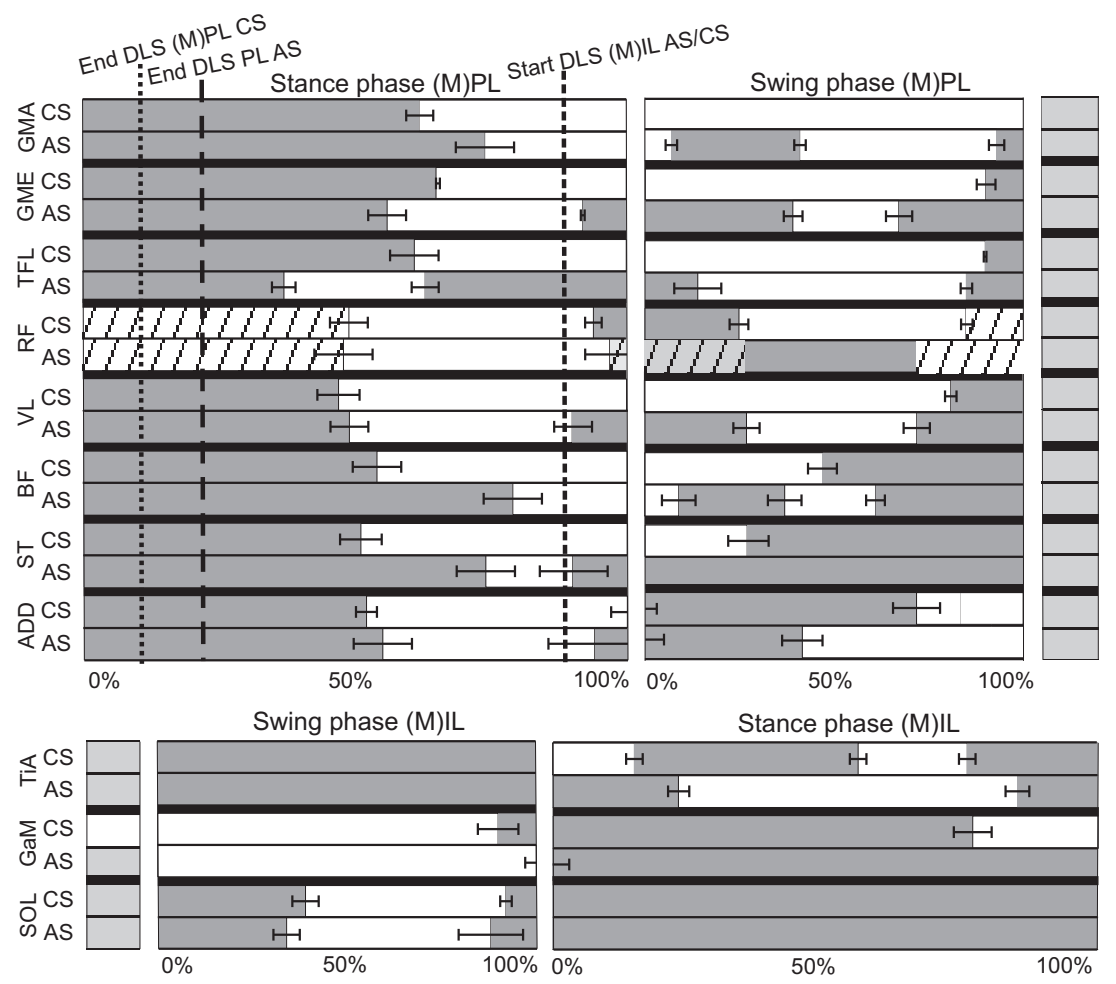

Figure 4.6: The average muscle activity of all muscles, for controls and amputees, as a percentage of the prosthetic stance and swing phase for slope walking. In dark grey the muscle is "on", in white it is "off", in light grey the overlapping phases, in hatched white periods of cross-talk and in hatched gray possible cross-talk. For the stance phase $0 \%$ is IC of the (mimicked) prosthetic leg ((M)PL) and $100 \%$ is initial swing of the (M)PL. The end of the first double limb support ( $D L S$ (M)PL) before prosthetic stance of controls and amputees are indicated. The start of the second double limb support (DLS (M)IL) is also indicated, which is equal for controls and amputees. For the swing phase, $0 \%$ is initial swing of the (M)PL and $100 \%$ is IC of the (M)PL. Part of the activity in the overlapping (first) double support phase is added separately, in light grey, to start or end of the swing phases. The whiskers show the standard error of the mean. All upper leg muscles are measured at the (M)PL and all lower leg muscles at the contralateral (mimicked) intact leg ((M)IL). Activity of the (M)IL is scaled similarly, but to the phases of the contralateral leg. 


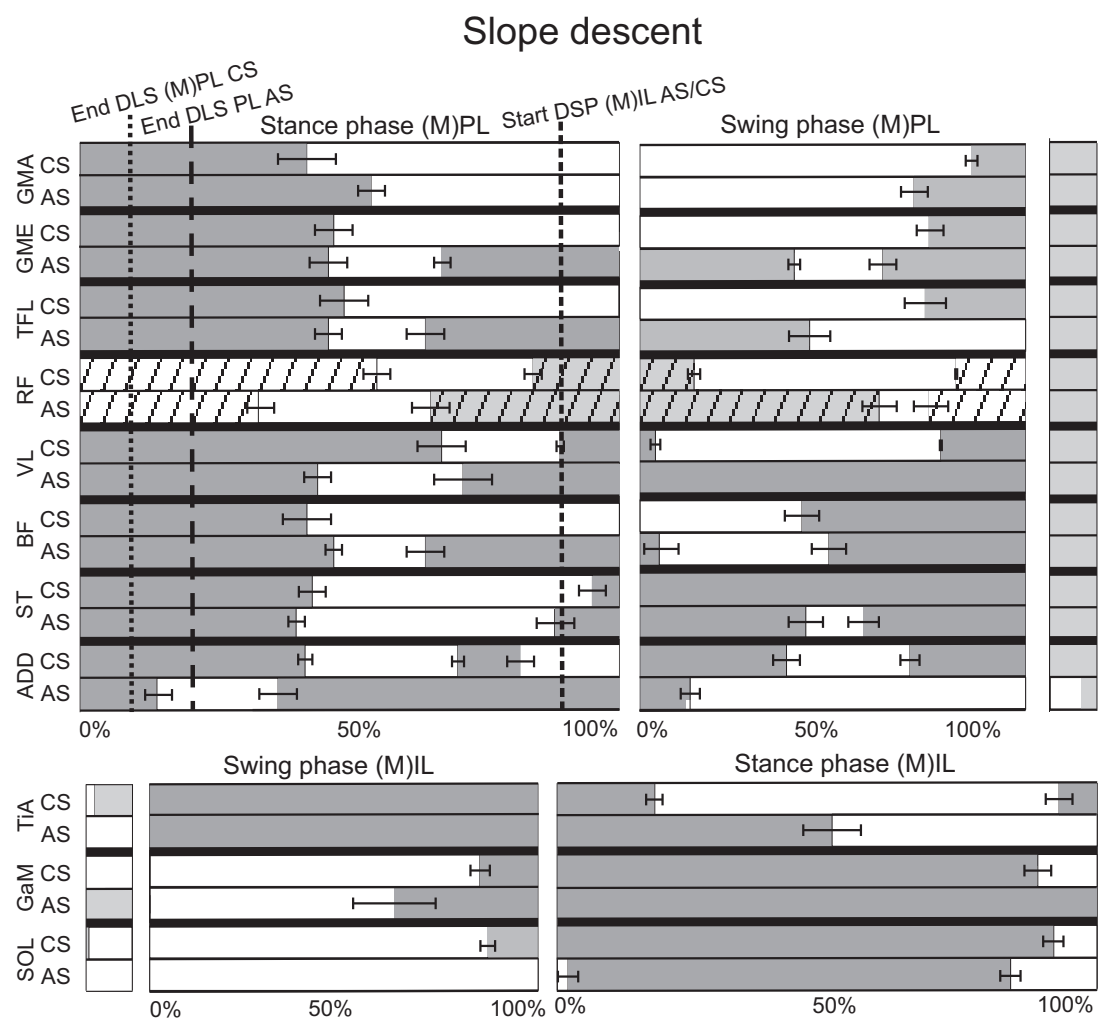

Figure 4.7: See figure 4.6

\section{Stair}

- Stair ascent - Most muscles of the controls are active for the entire stance phase (figure 4.8). The ST and the ADD only show activity in the first part of the stance phase. In amputees, only the GMa, GMe and the TFL show activity during almost the entire stance phase. The GMa and GMe show prolonged activity compared to controls. The other muscles show only activity in the first half of the stance phase. During the stance to swing transition, all upper and lower contralateral leg muscles of the amputees are active. In controls only the lower contralateral leg muscles, the ADD, the BF and ST are active. In the first half the swing phase the TFL and the RF of amputees show a second period of activity, which is not present in controls, the VL also shows this but for a shorter time period. The $\mathrm{BF}$ and the $\mathrm{ST}$ of amputees show activity during the complete swing phase, but in controls they only show activity during the last $60 \%$ of the swing phase. The rest of the muscle activity 
is comparable between amputees and controls.

In the lower contralateral leg the only difference is the SOL activity at the beginning of the swing phase in amputees, which is absent in controls.

- Stair descent - In the swing phase the only difference is that the VL start its activity earlier in amputees than in controls and the BF starts and ends its activity later in controls (figure 4.9). In the stance phase, muscle activity of the amputees is also similar to that of controls, although most muscle activity in amputees ends earlier in the stance phase. The ST and the ADD are active for the entire stance phase, which is different than in controls, where the ST is only active in the first part of stance and the ADD shows no activity at all.

The lower contralateral leg in controls the GaM shows activity from $30 \%$ till $70 \%$ of the stance phase, the Sol starts at the end of the swing phase and ends the activity at around $70 \%$ of the stance phase. In amputees both GaM and Sol start their activity around 30\% of the stance phase, and continue to be active for the rest of the step. 


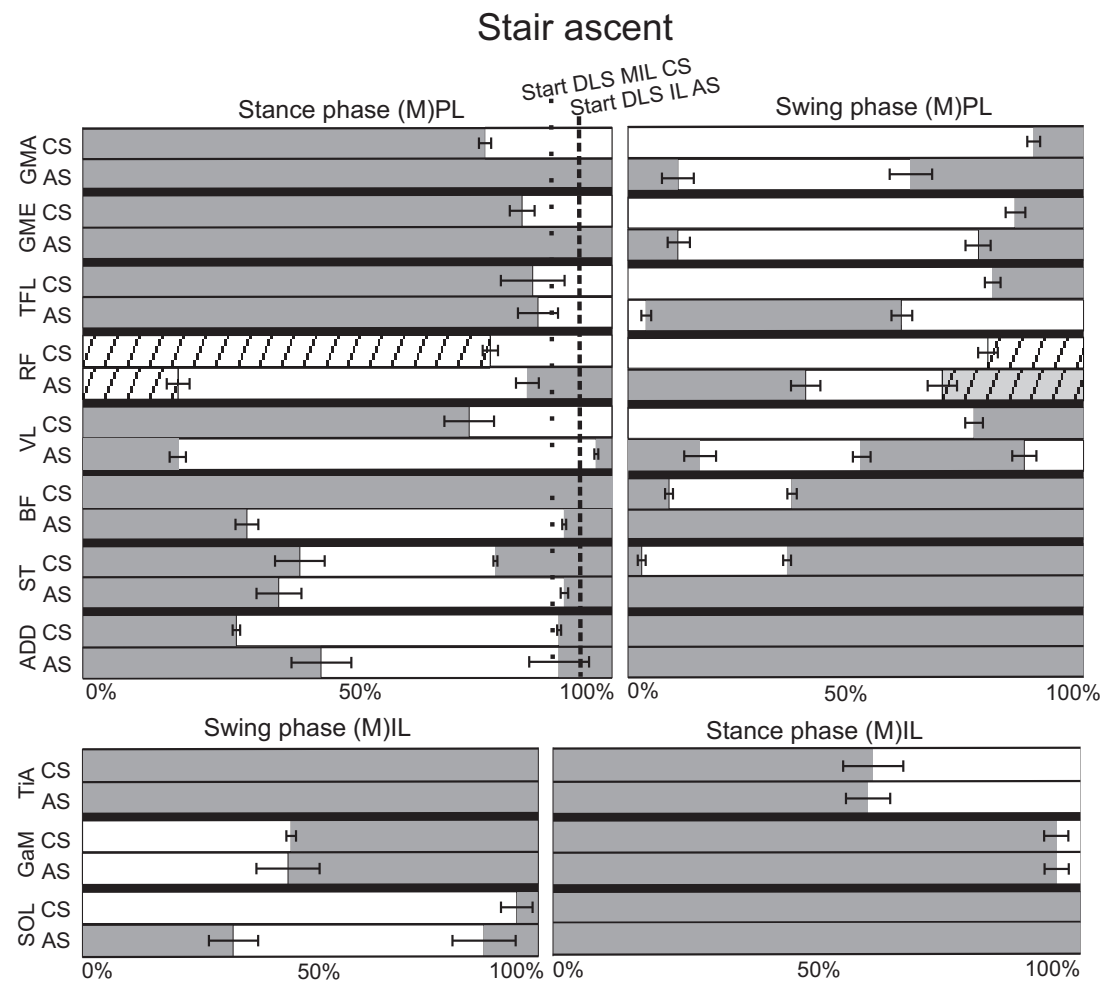

Figure 4.8: The average muscle activity of all muscles, for controls $(C S)$ and amputees (AS), as a percentage of the prosthetic stance and swing phase during stair ascent and descent. In dark grey the muscle is "on" and in white it is "off". Stair ascent: stance phase $0 \%$ is initial swing of the (mimicked) intact leg ((M)IL) and $100 \%$ is initial swing of the (mimicked) prosthetic leg ((M)PL). Double limb support (DLS) is indicated. For the swing phase, $0 \%$ is initial swing of the (M)PL and $100 \%$ is IC of the (M)PL. Stair descent: swing phase $0 \%$ is initial swing of the (M)PL and $100 \%$ is IC the (M)PL. For the stance phase, $0 \%$ is $I C$ of the (M)PL and $100 \%$ is IC of the (M)IL. 


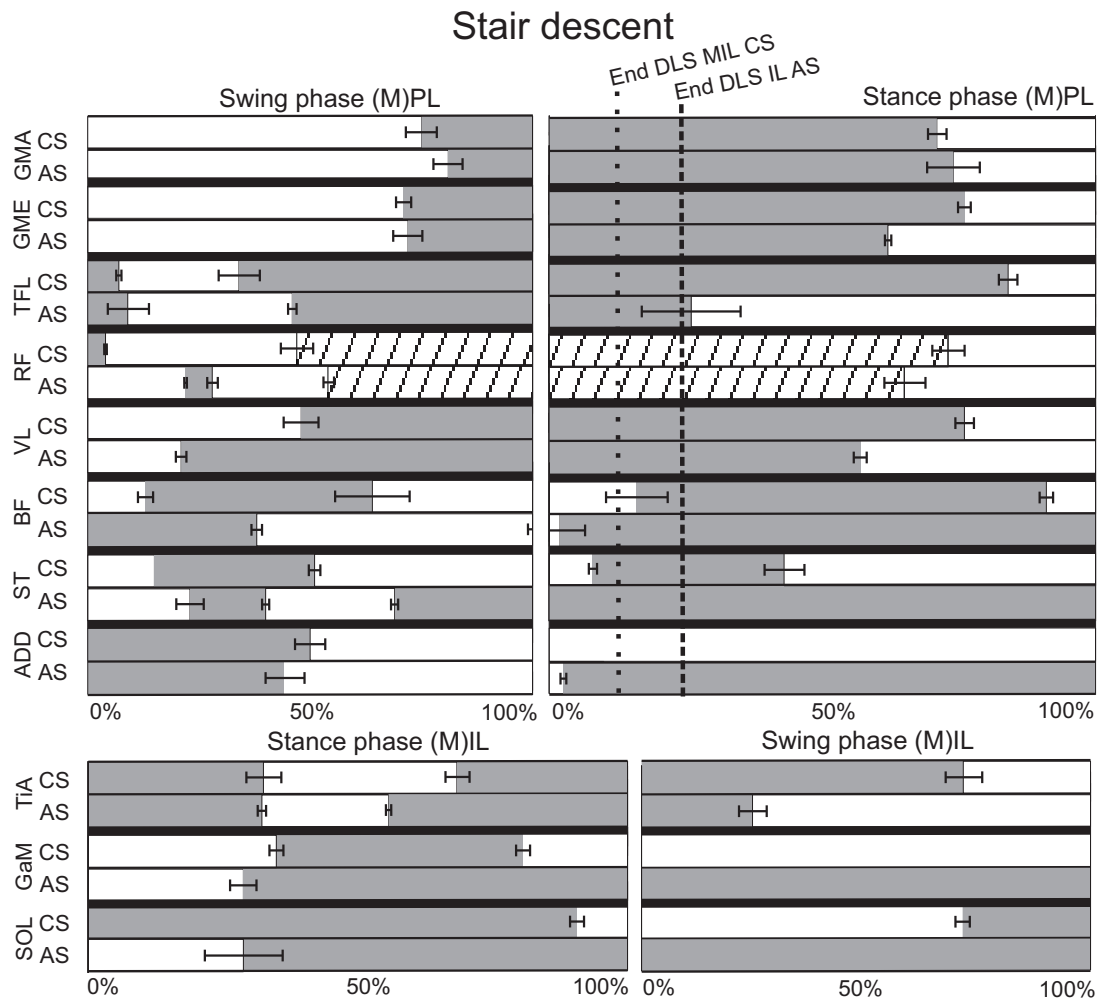

Figure 4.9: See figure 4.8

\section{Variability}

Calculated over all muscles there was no significant difference in VR between controls and amputees for slope ascent and descent. The average VR was 0.56 (0.05) for controls and 0.63 (0.11) for amputees in slope ascent. For slope descent the average VR was 0.54 (0.03) for controls and $0.71(0.05)$ for amputees. Figure 4.10 shows the VRs per muscle for slope ascent and descent.

The VR was significantly higher in amputees than in controls for stair ascent $(P=0.042)$ and stair descent $(P<0.001)$. The average VR was $0.59(0.12)$ for controls and $0.72(0.10)$ for amputees in stair ascent. For stair descent the average VR was 0.57 (0.1) for controls and $0.81(0.05)$ for amputees. Figure 4.11 shows the VRs per muscle for stair ascent and descent. 

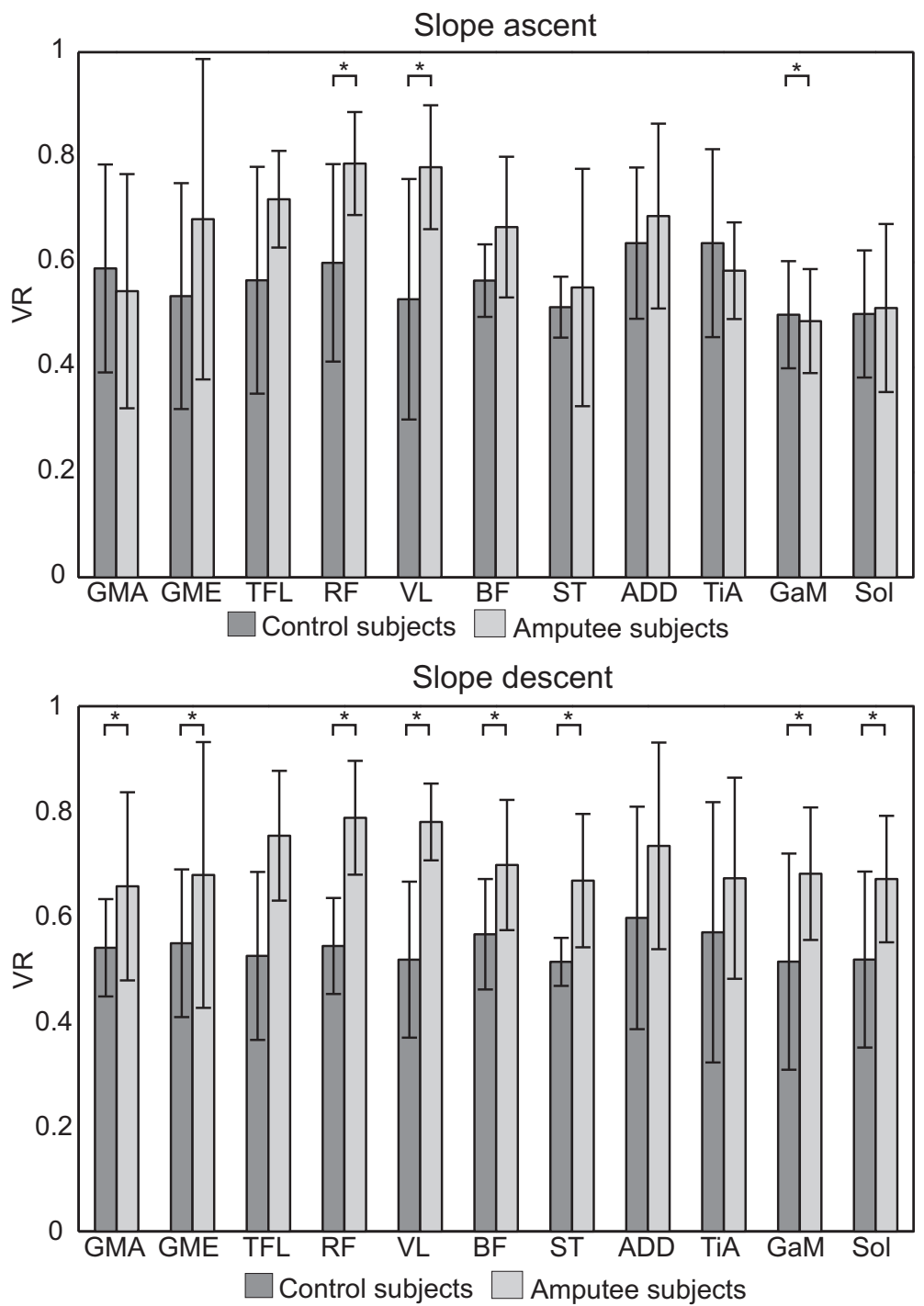

Figure 4.10: Variance ratios of all muscles of controls and amputees in slope ascent (top) and descent (bottom). Whiskers give one SD and * indicates significant differences.

\subsection{Discussion}

This study has shown the results of spatio-temporal, kinematic and EMG data during slope and SBS stair walking of controls and amputees. The results will now be discussed using the adaptations that are seen in amputees. All adaptations described are from the prosthetic leg of the 

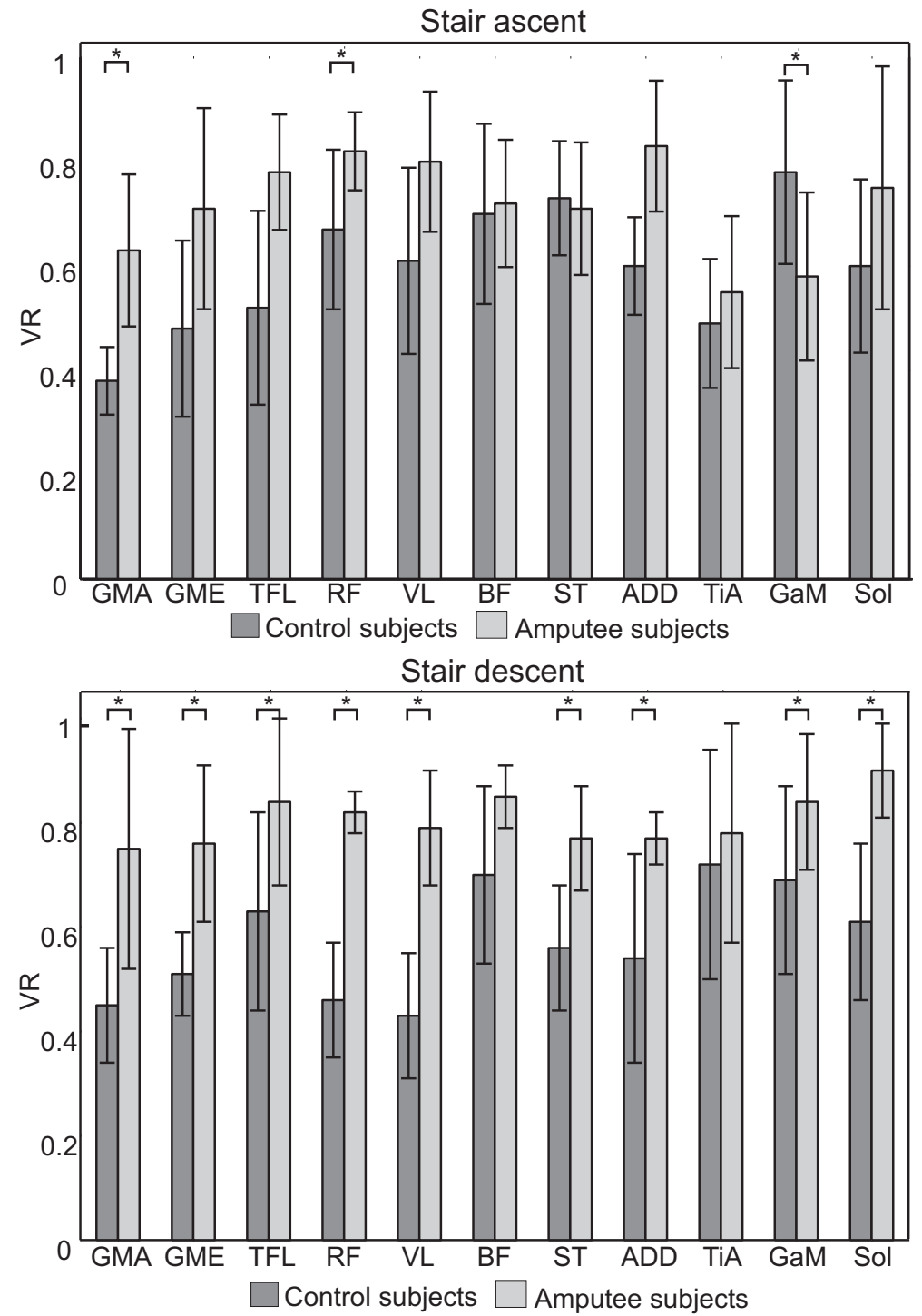

Figure 4.11: Variance ratios of all muscles of controls and amputees in stair ascent (top) and stair descent (bottom). Whiskers give one SD and ${ }^{*}$ indicates significant differences.

amputee, unless stated otherwise. 


\subsubsection{Slope}

The weight shift from the intact leg to the prosthetic leg is performed carefully by the amputee, as after the weight shift there is little room for corrections. Hip flexion is larger in slope ascent than in slope descent for both controls and amputees, which was not found by Vrieling at al. [23]. This may be caused by the difference in slope angle between that study $\left(5 \%, 2-3^{\circ}\right)$ and the current study $\left(10^{\circ}\right)$. We found that the initial double support phase is twice as long as in controls and as in level waking in amputees $[26,117]$. Walking speed for slope ascent and descent was significantly lower in amputees. For slope ascent previously EMG data as a percentage of the gait cycle for control subjects has been published for the VL, the ST, the SOL and the GaM. For these muscles the activity resembles that of the present study [124].

\section{Slope ascent}

Knee flexion in stance is almost absent, most likely due to the inability of the amputee to allow a knee flexion moment because of the possibility of a knee collapse. Although microprocessor controlled knees allow knee flexion during stance no clear differences were found between subjects with and without a microprocessor controlled knee.

The TFL activity at the beginning of the stance phase is most likely an adaptation to the lack of push-off. Due to a decrease in foot progression, the hip flexion moment is prolonged, which is seen in the prolonged activity of the GMa, the BF and the ST during the stance phase. The increased activity is also a way to ensure an extended knee during the stance phase. The decrease in forward foot progression results in a more posterior position of the center of mass. Therefore increased eccentric hip flexor activity is required, to prevent falling backwards during slope ascent. This may explain the increase in TFL activity during stance.

The increased activity of the VL, the RF, the BF and the ST at the end of stance and the beginning of the swing phase is likely aimed to increase socket fitting. The RF activity can also partly or completely be caused by cross-talk of the vastus intermedius [109]. By increasing the concentric contraction of the hip flexors (pull-off) the amputee is able to propel the leg into swing.

Duration of the GMe activity at the beginning of the swing phase is increased in both groups. To compensate for this, ADD also shows activity which allows the leg to move forward in a neutral position. Circumduction is the movent of the leg to abduction in the swing phase. 
Although hip abduction was decreased, three of the six amputees showed circumduction which is used by amputees to compensate for the reduced knee flexion. They may use a different adaptation mechanism to gain foot clearance, for instance more plantar flexion of the intact foot during stance, to achieve a virtual lengthening of the intact leg. Plantar flexion was not measured, therefore this mechanism could not be evaluated. However, the plantar flexors of the contralateral leg showed increased activity duration at the end of the stance phase. This may be used to compensate for reduced push-off and to achieve virtual lengthening.

\section{Slope descent}

Amputees have a decrease in progression over the foot, therefore the increase activity duration (eccentric) of the TFL during the stance phase may be an adaptation to prevent falling backwards. The TFL (and RF) show prolonged activity (concentric) at the start of the swing phase which may be used to propel the prosthetic leg forwards; the pull-off mechanism. To improve socket fitting the ST, VL and RF show increased activity, although the RF activity may also be cross-talk [109]. To compensate for the prolonged GMe activity, the ADD also shows prolonged activity during the swing phase [130].

For the lower contralateral (intact) leg the differences are mainly caused by a adaptation in foot positioning. The GaM shows earlier activity onset, which in combination with the TA stabilizes the foot. Plantar flexing the intact foot at the end of the swing phase allows the amputee to land on the foot as early as possible and reduce the single stance phase of the prosthetic limb.

\subsubsection{Stair ascent}

Horizontal velocity in SBS stair ascent in controls is comparable to that in the study by Reid et al. [128], but only half the velocity as in SOS stair ascent [128]. The velocity of amputees was lower, but not significantly.

The shorter prosthetic stance phase and the associated shorter swing phase of the intact limb in amputees indicates that the amputee performs a relatively faster motion of the intact limb to the next step compared to controls. The following double support phase is also shorter in amputees than in controls, indicating that weight shift to the intact leg is performed faster. Subsequently, relatively more time is taken to lift 
and place the prosthetic leg onto the next step. This coincides with the common concept that amputees have the tendency to stand longer on their intact leg than on the prosthetic leg $[24,26]$.

After weight shift of the intact limb to the prosthetic limb there is little room for corrections by the amputee. Therefore the amputees will try to keep the weight as much above the prosthesis as possible during the single support phase, which may explain the reduced hip extension. The abduction almost completely takes place in the swing phase and is therefore considered as circumduction, which is only seen in amputees. This is to compensate for the almost absent prosthetic knee flexion. This is likely caused by the lack of push-off in the prosthetic limb, which normally contributes to achieving knee flexion during swing. Amputees may also choose to lift the leg fully extended, to be sure the leg is also fully extended when it is placed on the next step.

To prevent a knee unlock, amputees will use the hip extensors to keep the knee in extension during the stance phase. This explains the prolonged activity shown by the GMa and GMe showed amputees. The RF activity at the beginning of the stance phase for both controls and amputees is most likely cross-talk [109]. The VL of amputees shows only a short period of activity at the beginning of the stance phase compared to controls, but in amputees the VL has no function during this period. The BF shows a shorter period of activity in amputees, similar to the ST.

At the start of the swing phase the amputee will need to increase the socket fitting. Activation of the ADD, the ST, the BF during the entire swing phase is most likely to increase socket fitting. The VL and RF activity at the start of the swing phase (and the end of the stance phase) is also likely to improve socket fitting and the RF will also flex the hip. Due to the lack of push-off, amputees will increase TFL activity as pulloff of the prosthesis.

The only difference at the contralateral leg is the SOL activity at the beginning of the swing phase. The explanation for this finding is unclear. However the co-contraction of the SOL and TiA might increase ankle stiffness allowing a quick response in case of disturbed balance. 


\subsubsection{Stair descent}

The horizontal velocity of SBS stair descent in controls in the current study is comparable to that measured by Reid et al. [128]. The SBS stair descent velocity in amputees not significantly lower.

Due to the lack of knee flexion in most amputees they circumduct the hip to get sufficient foot clearance. The prosthetic leg is slowly lowered and the foot carefully placed. This can be seen in the prolonged stance phase of the intact leg in amputees compared to controls. The reduction of hip flexion and the absence of knee flexion in the prosthetic swing phase in 4 of the amputees can also be explained by this careful placement of the prosthetic leg. By keeping the leg straight the amputee avoids a knee collapse when placing the weight on the prothesis. The two amputees that did have knee flexion in swing were both experienced walkers and had a microprocessor controlled knee which allows them to land on an unlocked knee without the risk of a collapse.

After foot contact the weight is slowly shifted. Little adjustments can be made to the posture at this stage, therefore this double support phase is significantly longer in amputees than in controls.

To ensure socket fitting, the VL, BF, ST and ADD are active during the (start of) the swing phase. The rest of the muscles show a similar pattern as in controls. The activity seen in the RF is most likely all due to cross-talk [109]. At the swing to stance transition most upper leg muscles of the amputees become active again. For the GMa, GMe, TFL, $\mathrm{RF}$ and VL this is also seen in controls. At the transition the amputee needs to have a fully extended leg, to prevent a knee collapse. The activity seen in the ADD, ST and BF can be explained by the tendency of the amputee to keep the knee in extension by "pulling" the hip in extension.

At the contralateral intact leg the amputee will try to control the drop down with the intact leg during the stance phase as much as possible. This explains the co-contraction of the SOL, the GMa and the TA in the amputee in the second half of the stance phase of the intact leg. This stiffens the ankle allowing it to control the drop down. In the swing phase of the intact leg, the amputee will try to reach the lower step as soon as possible, plantar flexing the ankle will allow earlier contact with the lower step. The TiA is therefore deactivated earlier and the plantar flexors are active during the complete swing phase of the intact leg, to pretension the ankle and allow immediate weight acceptance at foot contact. 


\section{Variability}

Even though electrodes were placed according to the SENIAM standards, due to the amputation some muscles may have a different orientation. Together with the cleavage of muscles this may have led to a non-optimal electrode placement and a higher VR in amputees. Socket fitting and rotations in the socket will also introduce noise and will affect the electrode positions. Muscles in amputees are also more active to increase socket fitting, which increases the baseline activity level. One subject complained that his socket was too large, his VR was on average higher than in the rest of the group. Apart from cleaved muscles the general walking pattern of amputees also seems to have more variation, which is also seen in the variability of the kinematic and spatio-temporal data.

\subsubsection{Methodological considerations}

The average age of the amputee group was higher (52.3) than in controls (23). Aging may effect the spinal cord activity and the walking speed, which again may cause a higher spread in muscle activity [21,113]. Two of the older subjects also had the highest VR, one of which still walked with a walking aid, which may also effect the VR [114]. Nevertheless EMG patterns were similar among the amputee group and SEMs comparable to controls.

In this study mainly data of the prosthetic leg was included. Amputees have however the tendency to adapt their walking pattern by increasing the use of the intact leg. Including more information on the intact would also give insight in the adaptations on the non-affected side.

In the experiments the stairs and slopes for controls and amputees were not exactly the same. The difference in stair height and depth were however both only $1 \mathrm{~cm}$, this may have lead to differences in the parameters between amputees and controls. When looking at the joint angle rotations $1 \mathrm{~cm}$ height difference may have a minimal effect on the data. It is however very likely that all differences found are explained by adaptations usually made by amputees. For slope walking also no obvious differences were seen between amputees and controls which may be explained by the difference in slope. The largest differences in walking pattern compared to normal walking seem to occur in slopes above $5 \%$ and both slopes used in this study are steeper than this [113]. 


\section{Further research}

Slope and stair walking of amputees showed to be highly asymmetric. However, striving towards gait symmetry may not be optimal for amputees. In this study (and others) we have seen that they seem to compensate a lot with the intact leg for their loss of control in the other leg $[26,116]$. Although slope walking in TFA is much more asymmetrical than slope walking in controls, the general gait pattern is very similar, also to level walking. In stair walking however, these patterns are changed dramatically, due to the inability of most amputee to negotiate stairs in a step-over-step manner. Therefore stair walking requires more adaptations from the amputee, which is mostly visible in the effort by the intact limb. It may not be necessary in rehabilitation to try to reestablish this symmetry, but rather train the amputee to establish an optimal walking pattern for their way of walking [131-133]. This may mean more training for the intact leg, to deal with the increase in control and load. Slope and stair walking should be included in standard rehabilitation programs, to provide amputees proper training and possibly reduce the load on the intact leg. Prosthetic fitting is also mentioned as a key-issue to improve prosthetic walking [133]. For further research it may also be interesting to include more data from the intact leg.

\subsection{Conclusion}

The general slope walking pattern in amputees resembles that of controls, but is much more asymmetrical. The first double support phase, where the prosthetic leg becomes the stance leg, is twice as long as in (normal) level walking (20\%). The stance phase of the intact leg also increases in both slope ascent and descent. Changes in joint rotations are similar for both groups. Amputees are however unable to increase knee flexion during swing.

EMG patterns are mainly different in the stance to swing transition, where more and prolonged activity is seen in amputees. These changes are most likely caused by the adaptations of the amputee to compensate for reduced foot progression, the inability to allow a knee flexion moment and to increase socket fitting and stability. Amputees also increase push-off at the intact side.

In stair ascent, step-by-step initiated with the intact leg, the stance phase of the intact leg $(67 \%)$ is longer but the double support phase $(6 \%)$ is shorter. Abduction and increased push-off by the intact limb are both strategies to ensure prosthetic foot clearance. Prosthetic knee 
flexion in stance was almost absent in amputees. In stance the hip muscles and the adductor are more active, which is replaced by only upper leg muscle activity during the swing phase, to ensure socket fitting. For stair step-by-step descent initiated with the prosthetic leg, the stance phase of the intact leg (70\%) and the double support phase (18\%) are longer. Hip abduction is increased, to compensate for the smaller knee flexion $\left(2^{\circ}\right)$ and ensure foot clearance. Muscle activity is mainly different in the swing to stance transition, more activity of the (former) knee extensors is seen. The intact leg also compensates for the loss of control over the prosthetic knee.

The variance in EMG in amputees is higher than in controls. Overall variability within the amputees is also larger, which may be caused by larger variability in the group, the walking pattern and the cleavage of muscles. 
Chapter 4 


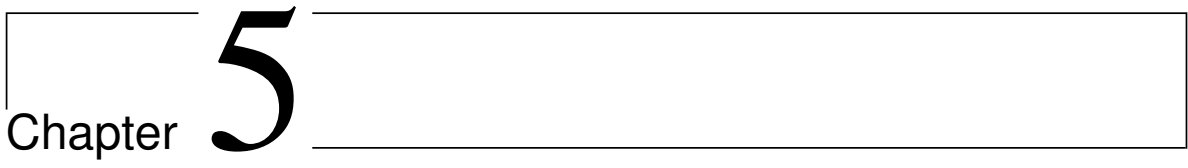

\section{Gait initiation detection in non-amputees ${ }^{1}$}

Gait initiation in transfemoral amputees (TFA) is different from nonamputees. This is mainly caused by the lack of stability and push-off from the prosthetic leg. Adding control and artificial push-off to the prosthesis may therefore be beneficial to TFA.

In this study the feasibility of real-time intention detection of gait initiation was determined, by mimicking the TFA situation in non-amputees. EMG and inertial sensor data was measured in ten non-amputees. Only data available in TFA was used to determine if gait initiation can be predicted in time to control an upper leg prosthesis to generate push-off and stability.

Initial swing and initial contact of the leading limb are important parameters to be detected, to control a prosthesis and to time push-off. The results show that initial swing and initial contact of the leading limb can be detected using EMG and kinematic data in non-amputees 130-260ms in advance. This leaves enough time to control a prosthesis.

Based on these results we hypothesize that similar results can be found in TFA, allowing for adequate control of a prosthesis during gait initiation.

\footnotetext{
${ }^{1}$ Published in: Gait and Posture 37(2):2013
} 


\subsection{Introduction}

Gait initiation in transfemoral amputees (TFA) is different from nonamputees $[23,25]$. In non-amputees it consists of two phases. First, preparations are made for the step execution [20,25,134]. During this phase postural adjustments are made, the center of pressure moves towards the leading limb and the body is tilted forward. Subsequently the center of pressure moves towards the trailing limb and the body is tilted further forward. The hip and knee of the leading limb start to flex and the ankle starts to dorsiflex to prepare for initial swing (IS), which is the end of the first phase. In the second phase the step is executed. It starts at IS of the leading limb and the body is tilted further forward. Muscles in the trailing limb stabilize the body, during swing of the leading limb, and generate push-off. The execution phase ends at initial contact (IC) of the leading limb $[20,23,25,134,135]$.

In TFA these two phases are similar, but the duration differs depending on which leg is leading, the prosthetic leg or the intact leg. It appears that TFA have the tendency to stand on their intact leg for as long as possible and load the prosthesis only briefly $[23,25,135]$.

Artificial push-off of an upper leg prosthesis during gait initiation may be beneficial, to allow a more natural process and reduce effort needed from the intact leg [23]. However, gait initiation must be predicted, because timing of push-off is very important. Push-off in gait is described as the part of the gait cycle which begins at onset of ankle plantar flexion and ends at IS [136]. Starting push-off too early will propel the amputee backwards. Starting too late will dissipate push-off or even cause a stumble.

To provide control inputs for supported prosthetic gait initiation, the beginning and end of the execution phase, IS and IC of the leading limb respectively, need to be detected for both leading limb conditions. If in amputees the prosthetic leg is leading, the prosthetic knee should flex at IS and be ready to take the load at IC. When the intact leg is leading, the prosthesis should provide push-off $[21,134]$.

For the detection of gait initiation several sensors may be used like gyroscopes and accelerometers, but also electromyography (EMG) from the remaining muscles. EMG of gait initiation in non-amputees was measured in several studies but primarily at the lower leg $[106,134,137]$. 
EMG activity in amputees during gait has been measured in a few studies and is comparable to that of non-amputees $[27,97,138]$. EMG during gait initiation in TFA has not been studied previously.

Inertial sensors have frequently been used to assess gait. However, few studies were found that used inertial sensors to assess gait initiation [139]. Most studies used a combination of an optical position measurement system and force plates $[23,25,106,134,135]$.

The authors found no studies on real-time intention detection of gait initiation in (non-)amputees. We therefore studied gait initiation detection in non-amputees, before advancing to TFA, but mimicking the TFA situation. We used data which can be measured in TFA, i.e. upper leg muscle activity and inertial sensors. Therefore the data can be used for upper leg prosthesis, lower leg prosthesis or even orthosis. In these applications the need for stability and control is desired in order to improve gait initiation and gait $[23,25,139-141]$.

The goal of this study is to determine if gait initiation can be detected from EMG of the upper leg muscles and/or inertial sensors. Detection should be sufficiently early to eventually support gait initiation in upper leg prostheses users. The current study was performed in non-amputees.

\subsection{Methods}

\subsubsection{Participants}

Ten healthy volunteers participated in the study, none of them had a history of lower limb injuries, neurodegenerative diseases or any skin conditions. The experiments were approved by the local Ethics Committee and an informed consent was obtained before the experiments.

\subsubsection{Measurements}

Kinematic data was measured $(100 \mathrm{~Hz})$ using 2 inertial sensors from Xsens (Enschede, Netherlands), with 3D accelerometers, gyroscopes and magnetometers. Electrodes were placed according to the SENIAM standards [100]. On each muscle two self adhesive electrodes (Kendal, H93SG, Tyco healthcare, Germany) were placed as closely together as possible. EMG measurements were performed with a 16 bipolar channel Portisystem from TMSi (Oldenzaal, Netherlands) at a sample frequency of 
$2048 \mathrm{~Hz}$.

Nine muscles were measured, due to a limited number of available EMG channels and to mimic the prosthetic situation. The upper leg muscles and inertial sensors were placed on one leg, which is the "mimicked prosthetic leg". The measured upper leg muscles are: the m. gluteus maximus (GMa), m. gluteus medius (GMe), m. tensor fasciae latae (TFL), m. rectus femorus (RF), m. vastus lateralis (VL), m. biceps femoris $(\mathrm{BF})$. In five subjects one extra muscle, the $\mathrm{m}$. erector spinea (ES), was measured.

At the lower leg on the contralateral side the $\mathrm{m}$. tibialis anterior (TA), m. gastrocnemius medialis (GaM), m. soleus (Sol) were measured, for reference purposes. This is the "mimicked intact leg". In five subjects the mimicked intact leg was the dominant leg and in the other five it was the non-dominant leg.

Footswitches, placed mid-heel and at the first metatarsal head of each foot, gave information about IC and IS. Subjects wore their own low-heeled shoes. Figure 5.1 illustrates the placement of the inertial sensors and EMG electrodes.

To synchronize EMG, footswitches and inertial sensors a synchronization pulse was given at the start and end of each measurement which was visible in all data sets.

\subsubsection{Procedures}

For the gait initiation experiments the subjects were required to stand upright with their weight equally distributed on both feet, the initial posture. Data recording was started. After five seconds in the initial posture the subjects were asked to press the synchronization button (sync) and start walking. After five paces they were asked to stop, turn around, return to the initial posture, wait 2-3 seconds, press the sync and walk back. This was repeated four times within each measurement. Two measurements were performed for each leading limb condition, 16 trials per condition.

In addition a postural sway measurement was performed, a forward and backward swaying motion, without falling forward or backward. This was used to calculate the thresholds for the inertial sensors for gait 
initiation detection.

\section{Sensor to body calibration}

The inertial sensors express their data in the sensor coordinate $\operatorname{system}(\vec{s})$. Two calibration exercises were performed to convert this data to the body coordinate $\operatorname{system}(\vec{f})$, using the rotation matrix $\left(R_{f s}\right)\left(\vec{f}=R_{f s} \vec{s}\right)$. In short the calibration of the lower leg was as follows. The subject stood upright, whereby the body z-axis equals the gravity vector which can be described in the sensor coordinate system. Subsequently the subjects flexed the knee five times to about 90 degrees, where the knee is the body y-axis, allowing this axis to be defined in the sensor coordinate system. The $\mathrm{x}$-axis is subsequently obtained by a cross product of the $\mathrm{y}$ and $\mathrm{z}$ axis. A similar procedure was followed for the upper leg sensor, using the squat as calibration exercise. $[101,142]$

This data was subsequently low-pass filtered at $10 \mathrm{~Hz}$. Finally the axis with largest amplitude was used for further analysis. For the angular velocity this was the body y-axis, for the acceleration it was the body z-axis (see figure 5.1).

\subsubsection{Data analysis}

EMG data analysis was performed in two parts. First the linear envelopes of the ensemble averages were calculated, to determine which muscles show a clear change in activity before IS or IC of the leading limb. Secondly, from the selected muscles the onset or offset timings were determined.

The EMG data was first high-pass filtered using a 2nd order Butterworth filter with a cut-off frequency of $20 \mathrm{~Hz}$ [80]. To calculate the linear envelopes the data was subsequently rectified and low-pass filtered with a 2nd order Butterworth filter at $9 \mathrm{~Hz}$ [80]. To calculate the timings, the high-pass filtered data was low-pass filtered at $500 \mathrm{~Hz}[80]$.

Onsets of the selected muscles were calculated using a log-likelihoodratio test (AGLR), as described by Staude $[78,143]$. This algorithm is suitable for real-time EMG onset or offset detection. The window-size used for the detection was $20 \mathrm{~ms}$, the threshold of the algorithm for on-off detection was set at $20[78,143,144]$. 


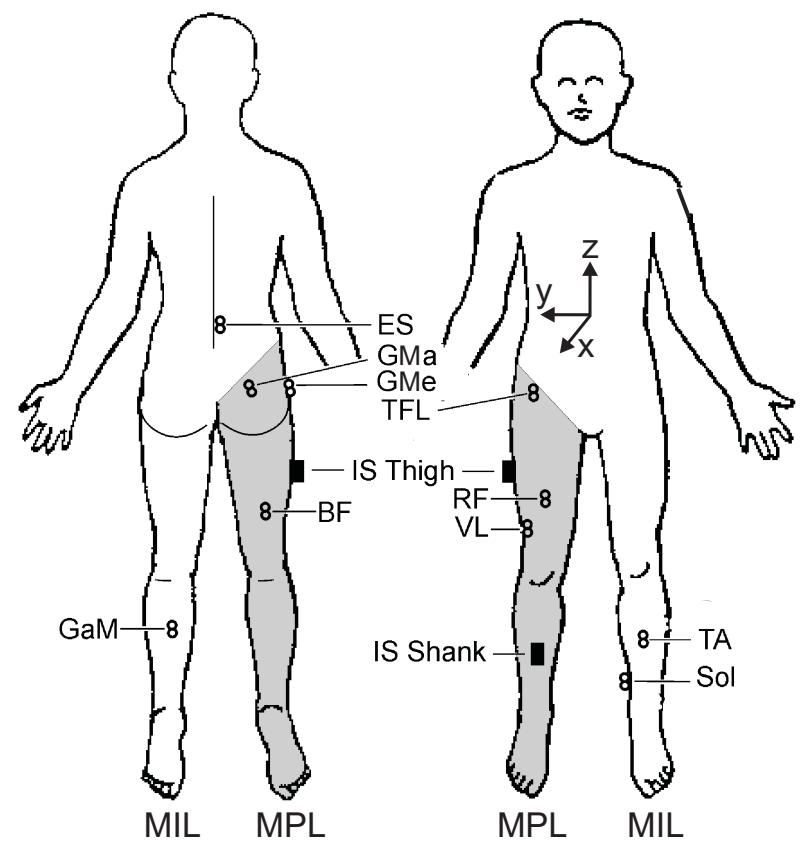

Figure 5.1: Placement of the inertial sensors (IS) and EMG electrodes on the on the body. One leg mimickes the prosthetic leg (MPL, in grey). At this leg all the upper leg muscles were measured and the inertial sensors were placed at the upper and lower leg. At the other leg, the mimicked intact leg (MIL), only the lower leg muscles were measured for reference.

The different phases of gait initiation were determined using the footswitch data. Push-off takes place between IC of the trailing limb, which is the onset of plantar flexion, and IS of the trailing limb [136]. For both leading and trailing limb IS and IC were determined. The data of each measurement was subsequently separated into trials and the trials were aligned at IC of the leading limb. From the aligned trials the ensemble averages were calculated per subject.

The intra subject variability of the EMG trials was determined using the variance ratio for each subject and muscle for the preparation and the execution phase $[106,107]$. The variance ratio is the variance of the data between gait initiation cycles normalized to the total variance. The lower the score the higher the repeatability. Differences between the preparation and execution phase were analyzed using a paired t-test with a p-value of 0.05 and Bonferonni corrections [106]. 
Postural sway measurements were performed to determine thresholds for the kinematic data after calibration, to decide if the subject was performing postural sway or was initiating gait. If the data from the measurements exceeded the sway thresholds, then IS or IC of the leading limb could be detected.

\subsection{Results}

\subsubsection{Ensemble Averages}

Figure 5.2 shows a typical example of the ensemble averages of the upper leg muscles and the inertial sensors, of one subject where the mimicked prothetic leg was leading (left) and where the mimicked intact leg was leasing (right). The arrows show the muscles and inertial sensors that have consistent activity changes before IS or IC of the leading limb for all subjects.

The variance ratio of the different muscles in the preparation and execution phase are shown in figure 5.3(b). The execution phase is significantly better reproducible compared to the preparation phase in case the mimicked prosthetic limb was leading. The muscles that can be used for the detection of gait initiation show generally a lower variance ratio than the other muscles, but this is not significant. The standard deviations, the between subject variation, are large in some cases but not differ between the different conditions.

\subsubsection{Timings}

IC of the leading limb was detected in all cases and was used as a reference measure for all timings, because IS of the leading limb was not detected in all trials. This was due to inaccurate or missing foot-switch data. Some trials were excluded, because subjects started walking with the wrong leg or no detection took place at all. The number of subjects and the percentage of trials included in the calculation of the timings are specified in table 5.2 .

Results for the timings of the footswitches can be found in table 5.1. Total push-off time (SD) was $285 \mathrm{~ms}$ (75), starting 166ms (66) before and ending $125 \mathrm{~ms}$ (38) after IC of the leading limb.

Table 5.2 shows the on and offset timings of the upper leg muscles before IS or IC of the leading limb in in all subject. Table 5.2 also shows the 

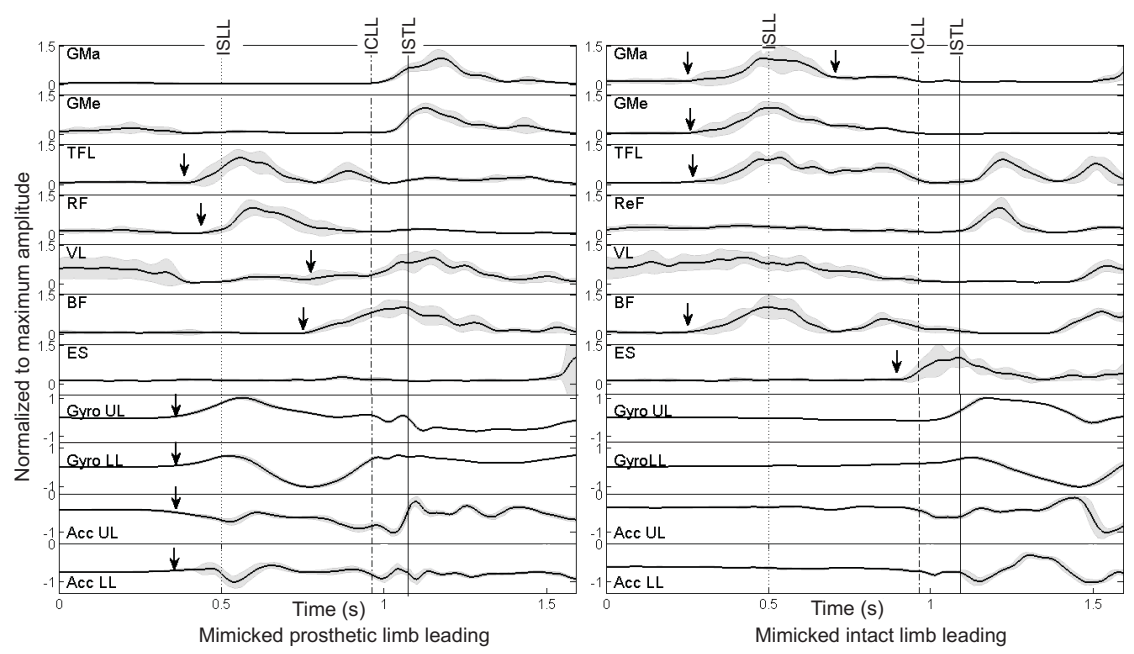

Figure 5.2: Muscle activity of the upper leg muscles and inertial sensor data of the mimicked prothetic limb (MPL) during gait initiation. The ensemble average is taken over 16 trials of one typical subject. Thick black lines indicate the average activity and the grey surface indicates plus and minus one standard deviation. The vertical lines indicate the events: IS leading limb, IC leading limb and IS trailing limb respectively. On the left, where the MPL was leading, activity changes are seen before IS of the leading limb in the TFL, the RF and the inertial sensor data and in the VL and BF before $I C$ of the leading limb. On the right, the mimicked intact leg was leading activity changes are seen in the GMe, the GMe, the TFL and the BF before IS of the leading limb. The GMa and ES show activity changes before IC of the leading limb.

detection of IS of the leading limb using inertial sensor data, which was only possible when the prosthetic leg was leading.

\section{Mimicked prosthetic leg leading}

The TFL and the RF showed activity onset in the ensemble averages, which is confirmed by the average onset of $129-199 \mathrm{~ms}$ before IS of the leading limb. The VL and the BF showed onset of activity about $150 \mathrm{~ms}$ before IC. Accelerometer and gyroscope data exceeded the sway thresholds 160-260ms before IS. IC could not be predicted from the kinematic data, it could however be detected. 


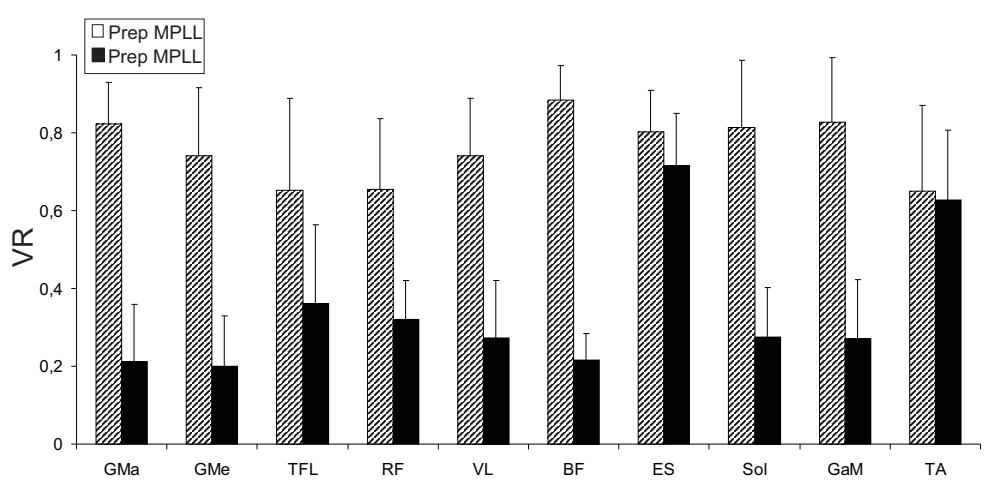

(a)

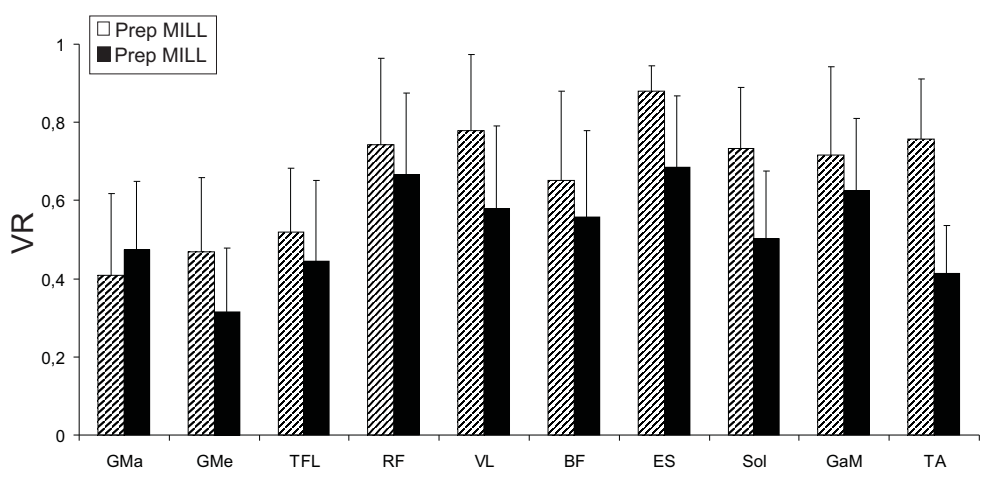

(b)

Figure 5.3: Variance ratios of all muscles averaged over all subjects with one standard deviation. The lower the score the better the reproducibility. The preparation phase shows a significantly lower reproducibility than the execution phase when the mimicked prosthetic limb was leading. 5.3(a) shows the reproducibility within subjects when the mimicked prosthetic leg was leading and 5.3(b) when the mimicked intact leg was leading.

\section{Mimicked intact leg leading}

For this condition, the GMa, the GMe, the TFL and the BF, could predict IS of the leading intact limb $200-224 \mathrm{~ms}$ in advance. IC of the leading limb was detected for this condition by the GMa (offset) and the ES (onset) $163-199 \mathrm{~ms}$ in advance. Kinematic data could not be used to predict IS or IC. 
Table 5.1: Timing foot contacts

\begin{tabular}{lllllllll}
\hline Time & HOLL & ISLL & HOTL & ICLL & ISTL & ICTL & $\begin{array}{l}\text { ISTL } \\
\text {-HOTL }\end{array}$ & $\begin{array}{l}\text { HOLL } \\
\text {-ISLL }\end{array}$ \\
\hline $\mathrm{ms}$ & -549 & -462 & -166 & 0 & 125 & 652 & 285 & 87 \\
$\mathrm{SD}$ & 49 & 49 & 66 & & 38 & 18 & 75 & 61 \\
\hline
\end{tabular}

(Timings determined using the footswitches averaged over all trials of all subjects. ISLL $=$ initial swing leading limb, HOLL $=$ heel-off leading limb, HOTL $=$ heel-off trailing limb, ISTL $=$ initial swing trailing limb, ICLL $=$ initial contact leading limb, ICTL $=$ initial contact trailing limb. A minus sign refers to the event taking place before HSLL. Timings are averaged over all subjects. SD $=$ standard deviation)

Table 5.2: Timings of the upper leg muscles

\begin{tabular}{llllllll}
\hline Leading & Muscle & ISLL(SD) & $\#$ & $\%$ & ICLL(SD) & $\#$ & $\%$ \\
limb & On/Off & in ms & sub & trials & in ms & sub & trials \\
\hline MIL & GMa On & $-220(97)$ & 10 & 90 & - & - & - \\
& GMe On & $-216(49)$ & 10 & 87 & - & - & - \\
& TFL On & $-224(62)$ & 10 & 95 & - & - & - \\
& BF On & $-200(89)$ & 10 & 81 & - & - & - \\
& GMa Off & - & - & - & $-199(70)$ & 10 & 78 \\
& ES On & - & - & - & $-163(67)$ & $5^{*}$ & 82 \\
\hline \hline MPL & TFL On & $-129(90)$ & 10 & 82 & - & - & - \\
& RF On & $-199(108)$ & 10 & 82 & - & - & - \\
& VL On & - & - & - & $-145(71)$ & 9 & 88 \\
& BF On & - & - & - & $-155(45)$ & 10 & 95 \\
\hline & sensor & ISLL(SD) & $\#$ & $\%$ & & & \\
\hline MPL & Acc UL & in ms & sub & trials & & & \\
& Acc LL & $-158(34)$ & 10 & 95 & & & \\
& Gyro UL & $-260(67)$ & 10 & 95 & & & \\
& Gyro LL & $-258(34)$ & 10 & 95 & & & \\
\hline
\end{tabular}

(A minus sign refers to the event taking place before the event. ISLL $=$ initial swing leading limb, ICLL = initial contact leading limb, $\mathrm{MPL}=$ mimicked prosthetic leg, $\mathrm{MIL}=$ mimicked intact leg, Acc $=$ accelerometer data, Gyro = gyroscope data, UL = upper leg, LL = lower leg, \# $\mathrm{i}$ the number of subjects included, $\%$ is the percentage of trials included, ${ }^{*}=$ the ES was only measured in 5 subjects.) 


\subsection{Discussion}

The goal of the experiments was to determine if gait initiation can be predicted in non-amputees using data which would be available in prosthetic users on the prosthetic side, e.g. EMG and kinematic data.

EMG of the upper leg muscles shows distinct patterns during gait initiation and was similar to that in other studies [106,134,137]. For both leading limb conditions EMG of the upper leg muscles showed activity changes 130-220ms before IS and IC. The RF and TFL can be used for the prediction of IS and the VL and BF for prediction of IC of the leading (prosthetic) limb. The GMa, the GMe, the TFL and the BF can predict IS and the GMa and ES can predict IC of the leading (intact) limb. Kinematic data could predict IS of the leading (prosthetic) limb, 158-260ms in advance.

\subsubsection{Mimicked prosthetic leg leading}

Previous studies showed that TFA have a tendency to start gait initiation with the prosthetic limb, because fewer adjustment strategies are needed to initiate gait with the prosthesis $[23,25]$. When the prosthesis is leading, the knee should flex at IS and extend at IC $[21,134]$. In TFA a short preparation and a long execution phase was seen if the prosthetic leg was leading compared to non-amputees [25]. But even if the preparation phase in TFA is half the duration of that in non-amputees the current results suggest there is still enough time to control the prosthesis.

Due to the lower reproducibility of EMG in the preparation phase, the prediction of IS needs the inertial sensor data. In microprocessor controlled knees some of these inertial sensors are already build in. IC can be predicted using the EMG data.

\subsubsection{Mimicked intact leg leading}

If in amputees the intact leg was leading the preparation phase was longer and the execution phase shorter compared to healthy individuals [25]. Due to the longer preparation there may be more time to detect IS of the leading limb in TFA compared to non-amputees.

Timing of push-off when the intact limb is leading, may need some consideration. To add push-off to prosthetic gait, IC and IS of the trailing limb need to be detected for correct timing $[21,134,136]$. However, if 
no ankle flexion takes place in the prosthesis IC and IS will occur almost at the same time. Detection of IC and IS of the leading limb will be more useful. The results show that four muscles are able to predict IS of the leading (intact) limb with good reproducibility. However, only the GMa has a high reproducibility in IC detection. IC of the leading limb may not need to be predicted (only detected) in this case, because push-off ends $125 \mathrm{~ms}$ after IC.

\subsubsection{Methodical considerations}

IS was not detected in all trials, the footswitches did not provide any information about the applied pressure. Furthermore, at the initial stance phase of gait initiation the weight of the subject shifts backwards a little which may unload the toe switches of the leading limb and therefore unloads the switches before actual IS.

The erector spinae was only measured in five subjects, during the experiments we found that the erector spinae may give valuable information on postural changes, therefore it was added later. Data of the ES may be used for detection of IC if the intact limb is leading, but the variance ratios were among the highest. For the final application it is therefore not useful.

In previous studies, duration of activity of some muscles in TFA was found to be a little longer than in non-amputees $[27,97,138]$. This should not be a problem for gait initiation detection in TFA, as long as clear changes in muscle activity can be detected before IS and IC of the leading limb. For offset detection of a muscle this may mean that less time is available prior to the event, but this was only relevant in the GMa if the mimicked intact limb was leading.

For longer residual limb lengths, amputation at the distal half of the upper leg, all the suggested muscles are likely to be available if myodesis of myoplasty has been performed. For short residual limb lengths however, some of the suggested muscles may not be available any more [27].

Although all data was processed in such a way that onset detection can be performed realtime, there is need for a decision algorithm. To implement control into a prothesis, similar results must be found in TFA and more activities should be analyzed. 


\subsection{Conclusions}

Detection of gait initiation from EMG of the upper leg muscles and kinematic data in mimicked amputee gait initiation was possible. Intention detection of gait initiation allows $130-260 \mathrm{~ms}$ for control of a prosthesis. However, further studies are needed to determine the possibilities to predict gait initiation in TFA. 
Chapter 5 


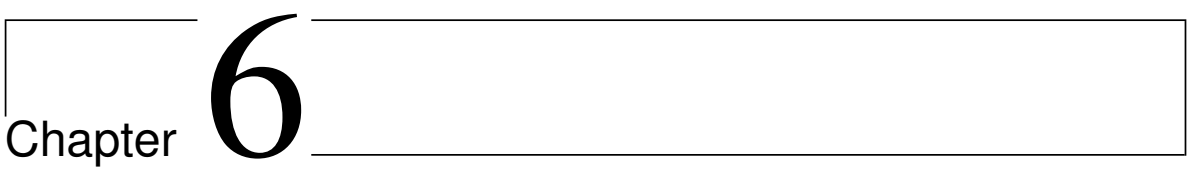

\section{Gait initiation detection in transfemoral amputees ${ }^{1}$}

In this study we determined if intention detection of gait initiation in transfemoral amputees can be useful for voluntary control of upper leg prostheses.

From six transfemoral amputees inertial sensor data and EMG were measured at the prosthetic leg during gait initiation. First, initial movement was detected from the inertial sensor data. Subsequently it was determined whether EMG could predict initial movement before detection based on the inertial sensors with comparable consistency as the inertial sensors.

From the inertial sensors the initial movement can be determined. If the prosthetic leg leads, the upper leg accelerometer data was able to detect initial movement best. If the intact leg leads the upper leg gyroscope data performed best. Inertial sensors at the upper leg in general showed detections at the same time or earlier than those at the lower leg. EMG can predict initial movement up to a 138ms in advance, when the prosthetic leg leads. Only one subject showed consistent EMG onset up to $248 \mathrm{~ms}$ before initial movement in the intact leg leading condition.

A new method to detect initial movement from inertial sensors was presented and can be useful for additional prosthetic control. EMG measured at the prosthetic leg can be used for prediction of gait initiation when the prosthetic leg is leading, but for the intact leg leading condition this is not expected to be of additional value.

\footnotetext{
${ }^{1}$ Accepted for publication in Gait and Posture.
} 


\subsection{Introduction}

The two phases of gait initiation in transfemoral amputees (TFA) are different from non-amputees $[23,25]$. In the first phase, preparations are made for the step execution; the weight is shifted to the trailing leg which ends at initial swing (IS) of the leading leg $[20,25,134]$. In TFA this first phase is short when the prosthetic leg leads (PLL), and relatively long when the intact leg leads (ILL), compared to non-amputees. [25] The second phase starts at IS and ends at initial contact (IC) of the leading leg. In TFA this phase is long when the PLL, but relatively short when the ILL, compared to non-amputees. [25]

If gait initiation can be predicted in TFA, the prosthesis can be controlled such that it is prepared for lifting of the prosthesis, in case the PLL. Prosthetic control during gait initiation may also provide a stable knee in case the ILL. If future prostheses can provide push-off, gait initiation detection may also become very useful.

Timing of push-off is very important and therefore accurate prediction of gait initiation is also important [102]. IS and IC of the leading leg are for both PLL and ILL important to be detected, to provide control inputs for supported prosthetic gait initiation. In non-amputees gait initiation could be predicted up to $260 \mathrm{~ms}$ in advance for both leading leg conditions, using electromyography (EMG) and inertial sensors [102]. A study by Zhang et al [36] showed detection of the beginning of the swing phase from stance to walking using EMG, in one amputee, up to $152 \mathrm{~ms}$ before the event. They used a custom made liner but did not mentioned which leg was leading.

To determine if inertial sensors or EMG at the upper leg are of additional value for prosthetic control we studied gait initiation detection in 6 amputees using inertial sensors and EMG, both from the prosthetic leg. No modification of the socket or liner was introduced. From this data we investigated a new method for gait initiation detection of the leading leg of TFA, using inertial sensors. Subsequently we determined if EMG provides additional information to the inertial sensing, and if gait initiation can consistently be predicted in TFA from inertial sensing and/or EMG. 


\subsection{Methods}

\subsubsection{Participants}

Six unilateral amputees participated in this study, three transfemoral amputees (TFA) and three through the knee amputees (TKA). Demographic variables of the amputees can be found in Table 3.1. Inclusion criteria were: have a unilateral TFA or TKA regardless of the reason for amputation; be between 18 and 70 years old; be a prosthetic user able to walk independently with or without a walking aid (K-level 2, 3 and 4). An informed consent was obtained before the experiments, and the study was approved by the local Ethics Committee.

\subsubsection{Measurements}

Footswitches, placed mid-heel and under the first metatarsal head of each foot, gave spatio-temporal information. Two inertial sensors (Xsens, Enschede, the Netherlands), placed at the frontal side of the upper and lower (prosthetic) leg, halfway between the hip and the knee and between the knee and the ankle. Kinematic data was measured at $100 \mathrm{~Hz}$. Subjects wore their own low-heeled shoes.

EMG registration was performed on eight upper leg muscles of the residual part of the prosthetic leg: gluteus maximus (GMa), gluteus medius (GMe), tensor fasciae latae (TFL), rectus femoris (RF), vastus lateralis (VL), biceps femoris (BF), semitendinosis (ST) and the adductor magnus (ADD).

Electrodes were placed according to the SENIAM standards [100]. Because normal anatomy is disturbed at the amputated side, EMG was checked prior to the measurements by selective contraction of the measured muscle. On each muscle two self adhesive electrodes (Ambu, BRS) were placed approx. $1 \mathrm{~cm}$ apart. EMG measurements were performed with a 16 bipolar channel Porti-system (TMSi, Oldenzaal, the Netherlands) at $2048 \mathrm{~Hz}$. A synchronization pulse (sync) at the start and end of each measurement was used to synchronize the Porti and Xsens systems.

\subsubsection{Procedures}

Subjects were required to stand upright, the initial posture. Data recording was started. After five seconds in the initial posture the subjects were asked to press the sync and start walking. After five paces they 
were asked to stop, turn around, return to the initial posture, wait 2-3 seconds, press the sync and walk back ( 1 trial). One measurement consists of 4 trials and two measurements were performed for each leading leg condition, 16 gait initiations per condition. In addition a stance measurement was performed where subjects were asked to stand in one spot for $30 \mathrm{sec}$.

\subsubsection{Data analysis}

Footswitch data was used to detect IC, which was detected in all trials and therefore used to overlap the trials [102]. The overlapped trials were subsequently cut into trials, from 2 sec before IC until IC.

Initial swing (IS) detected from the footswitches, was defined as the moment where both sensors under one foot lost contact with the floor. Initial movement (IM) was detected using the modulus of the $3 \mathrm{D}$ accelerometer and gyroscope data of the upper and lower prosthetic leg [145]. The modulus of the accelerometer data (acc-data) during quiet stance is $9.81 \mathrm{~m} / \mathrm{s}^{2}$, upon lifting of the leg a peak in the data is seen [145]. In the modulus of the gyroscope data (gyro-data) the forward body motion was clearly visible (figure 6.1). The inertial sensor data was expressed in the body coordinate system based on a sensor-segment calibration procedure as described by Wentink et al. [102]. The inertial sensor data, expressed in body coordinates, was subsequently low-pass filtered at 10 $\mathrm{Hz}$ with a second order, butterworth filter.

The thresholds for both detection methods of IM were determined from the stance measurements, because subjects were usually not standing completely still. The average of the moduli during stance was used as a baseline for IM detection, both acc-data and gyro-data had to be at least $100 \mathrm{~ms}$ within $1 \mathrm{SD}$ of the baseline before IM detection was attempted. The threshold for IM detection for both methods was: mean stance measurement $+5^{*} \mathrm{SD}$. This was the lowest threshold that did not detect any movements during stance. Both detections methods were analyzed for the upper and the lower limb to determine the most consistent, and the earliest detection of IM.

Detections of IM and IS were performed with respect to IC. Significant differences in timings were tested using the Mann-Whitney-Wilcoxon test with $\mathrm{P}<0.05$. Per leading limb condition the best method was selected first by determining the number of included trials and subsequently the consistency. 
EMG data were high pass filtered at $10 \mathrm{~Hz}$ and low pass filtered at $500 \mathrm{~Hz}$ with a second order butterworth filter and subsequently cut into trials, from $2 \mathrm{sec}$ before IC until IC. For on/off detection the data was rectified and integrated in a window of 20 samples, a post-processor of 4 windows, set the total detection time delay to $40 \mathrm{~ms}$.

The threshold for on/off detection was determined per muscle, per subject as the mean rectified and integrated 30 sec resting-EMG plus three times the SD. [104-106]

First the EMG on/offsets were determined per muscle, subject and trial with respect to IC. We subsequently preselected the muscles whereby EMG on/offsets were closest to IM. From those pre-selected muscles we calculated their timing with respect to IM, to determine if the on/offset per trial was before or after IM. For each subject we subsequently chose those muscles which met two criteria: 1 ) onset was before IM in all trials and 2) in total one trial was allowed to be excluded.

\subsection{Results}

Trial exclusion - For each leading leg condition there were 5 subjects that could perform the measurement, one subject was unable to perform ILL, another subject was unable to perform PLL. For the PLL condition 75 trials were included. Two subjects initiated one trial with the "wrong" leg of which one only performed 14 trials in stead of 16 due to fatigue. In one other subject in one trial no IC was detected using the footswitches, this trial was left out. For the ILL condition 73 trials were included, no trials were excluded, however one subject was only able to perform 9 trials.

\subsubsection{Detection of IS and IM}

$P L L$ - The upper part of table 6.1 shows the results of IS/IM detection from the PLL condition. Only two subjects showed IS in the footswitch data. Only the upper leg acc-data detection allowed all trials to be included, therefore the upper leg acc-data was used for IM detection for PLL. IM detection in the upper leg acc-data was significantly earlier in two subjects compared to the lower leg acc-data. IM detection using the upper leg acc-data was in one subject $260 \mathrm{~ms}$ earlier than IS, in the other there was no difference. Figure 6.1 shows an example of the 
detections.

$I L L$ - The lower part of table 6.1 shows the results of IS/IM detection for the ILL condition. Only the upper leg gyro-data allowed detection of IM in all trials in all subjects. Therefore the upper leg gyro-data was selected for IM detection for ILL. In four subjects these detections were significantly earlier than those at the lower leg. IM detection using inertial sensors was on average (range) $50 \mathrm{~ms}(-180-170 \mathrm{~ms})$ earlier than using footswitches and more trials were included when using inertial sensors. 


\begin{tabular}{|c|c|c|c|c|}
\hline & \# & $0 \stackrel{0}{-1} 0$ & $\stackrel{9}{\rightarrow}+\sigma \stackrel{10}{-} \stackrel{20}{=}$ & \\
\hline 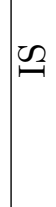 & 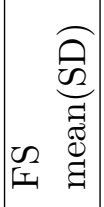 & 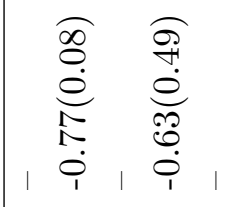 & 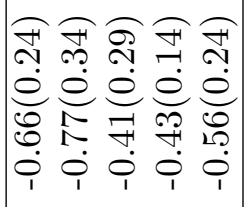 & 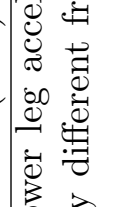 \\
\hline & \# & صـ & $\stackrel{0}{\because}$ or $\sigma \stackrel{\bullet}{\sim} \stackrel{0}{=}$ & \\
\hline & 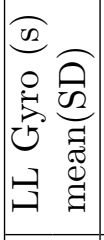 & 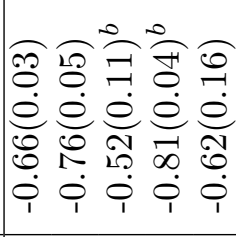 & 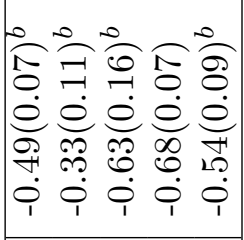 & 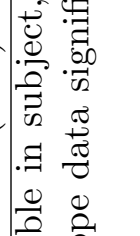 \\
\hline & \# & $\exists \stackrel{H}{-}=\stackrel{20}{\rightarrow} 0$ & 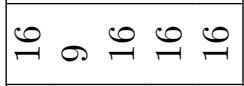 & \\
\hline & 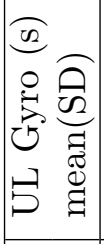 & 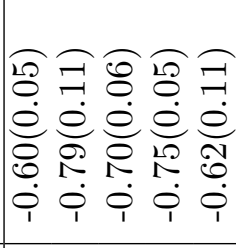 & 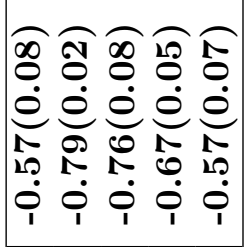 & 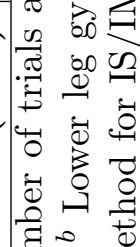 \\
\hline & \# & 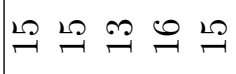 & $\infty \circ \infty \stackrel{0}{\sim} \stackrel{0}{=}$ & \\
\hline & 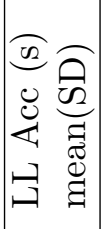 & 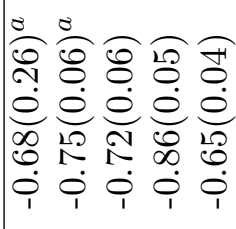 & 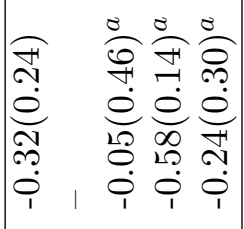 & 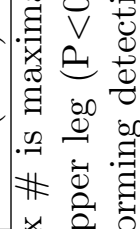 \\
\hline & \# & $\stackrel{20}{0} \stackrel{9}{\sim} 0$ & 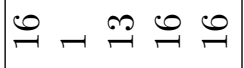 & \\
\hline & 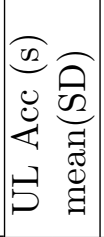 & 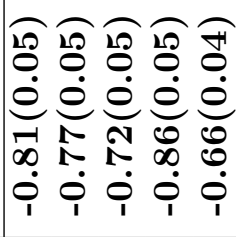 & 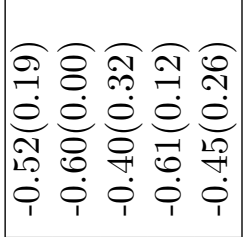 & \\
\hline & $\begin{array}{l}\stackrel{x}{u} \\
\ddot{g}\end{array}$ & $\stackrel{20}{\longrightarrow} \stackrel{0}{\longrightarrow}$ & $\stackrel{\bullet}{-} \sigma \stackrel{0}{\bullet} \bullet$ & \\
\hline & $\frac{\overrightarrow{0}}{3}$ & 安安䒧是是 & 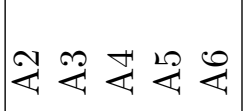 & \\
\hline & & 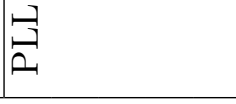 & & $z$ \\
\hline
\end{tabular}



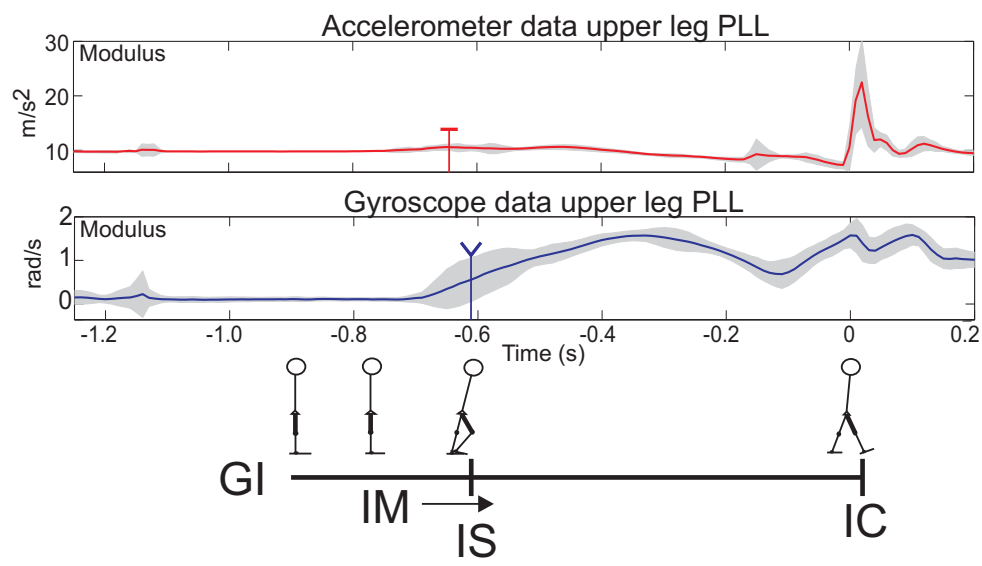

(a) PLL

Acc-data UL ILL

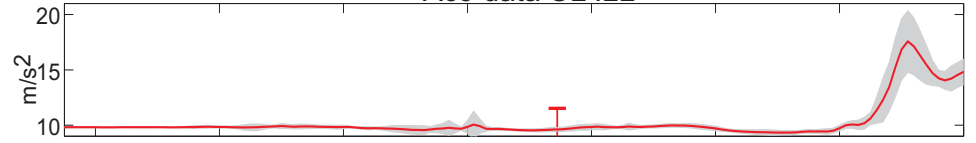

Gyro-data UL ILL

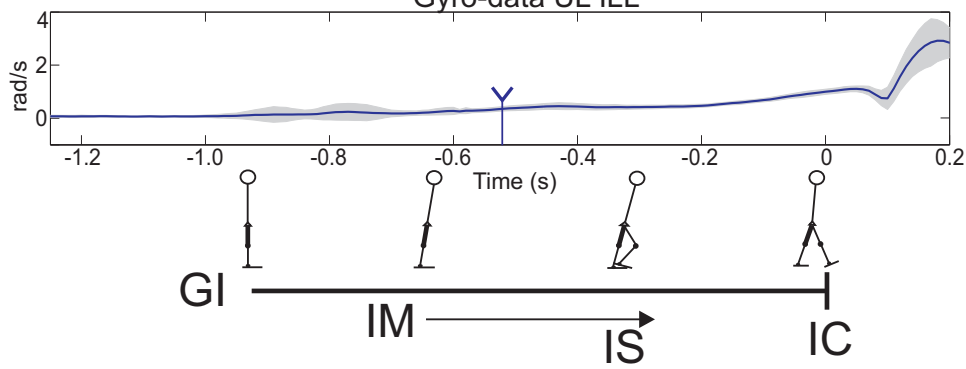

(b) ILL

Figure 6.1: An example of one representative amputee for detection of IM. (a) PLL condition (b) ILL condition. In red the ensemble average of the modulus of the upper leg accelerometer data of the prosthetic limb, in blue the average modulus of the upper leg gyroscope data of the prosthetic limb, the grey shaded areas are +/- 1 SD. Per leading limb condition a schematic overview of GI for that leading limb is presented. Vertical lines show the average detections from this subject, the gyroscope data $(Y)$ and the accelerometer data $(T)$. In both leading limb conditions IC of the leading leg occurs at $t=0$. 


\subsubsection{Detection from EMG}

Figure 6.2 shows an example of muscle on/offsets with respect to IC. In the first $500 \mathrm{~ms}$ some on/offset detections were found, but not in all trials. Subsequently all muscles are silent for $500 \mathrm{~ms}$, which was seen in both conditions for all amputees. Muscles with EMG onsets with a median before or at the average IM were pre-selected (example see fig. 6.2). From pre-selected muscles, per trial the onset with respect to IM was calculated (see figure 6.3). Onset detections between IM and IC occurring in all trials were rare. Offset detections occurring in all trials before IM or between IS and IC were also rare or with large SD. Therefore in the following analysis only the onset detections before or at IM were taken into account. Of those muscles meeting the two criteria, the average timing before IM and the SD were calculated (table 6.2).

$P L L$ - In four subjects at least one muscle was found that met the criteria, of which they had the TFL in common. The TFL had the lowest SD in all subjects and its onset was on average $78-140 \mathrm{~ms}$ before IM.

$I L L$ - In two subjects four muscles were found with EMG onset detection before IM. In the others the criteria were not met. One of the subjects shows detections with SD comparable to IM detections, the other one has SD between 137-155 ms. 
EMG on/off detections ILL

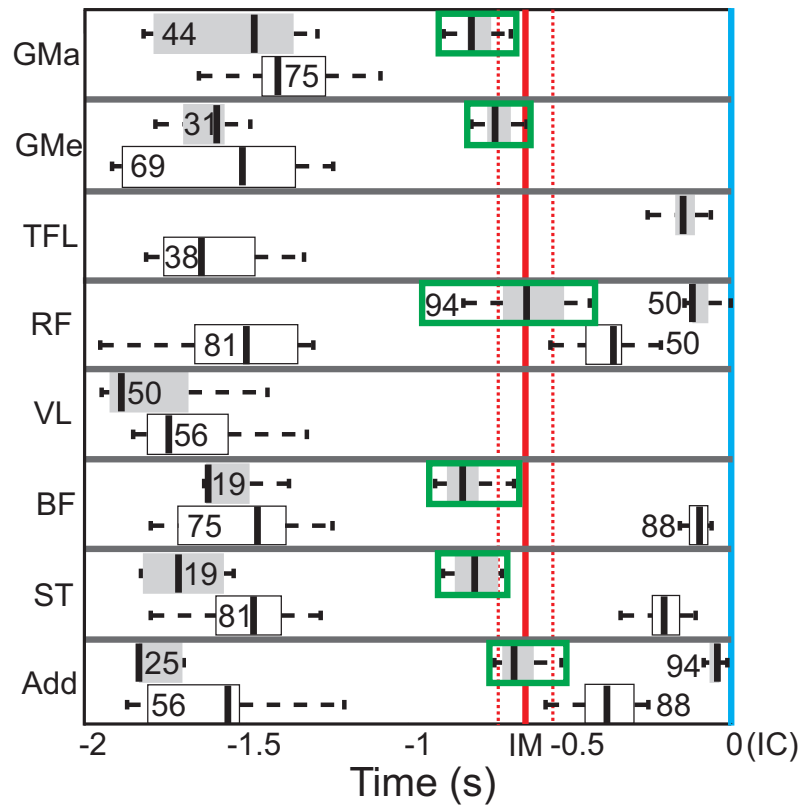

Figure 6.2: An example of the on/offset detections a representative amputee over time, from the ILL condition. The red vertical line indicates the average IM of this subject over all trials using detections from the modulus of the gyroscope data, the dotted lines are $+/-1 S D$. The blue vertical line is IC. White boxes are offset detections and in grey the onset detections. If a number is presented at the box, this is the \% of trials in which this detection was found, if no number is given the detection was found in all trials. The onsets marked in green are those closest to IM and with the required number of trials, they were preselected. In each boxplot the solid line represents the median, the box shows the 25 and 75 percentiles and the whiskers mark the complete range. 

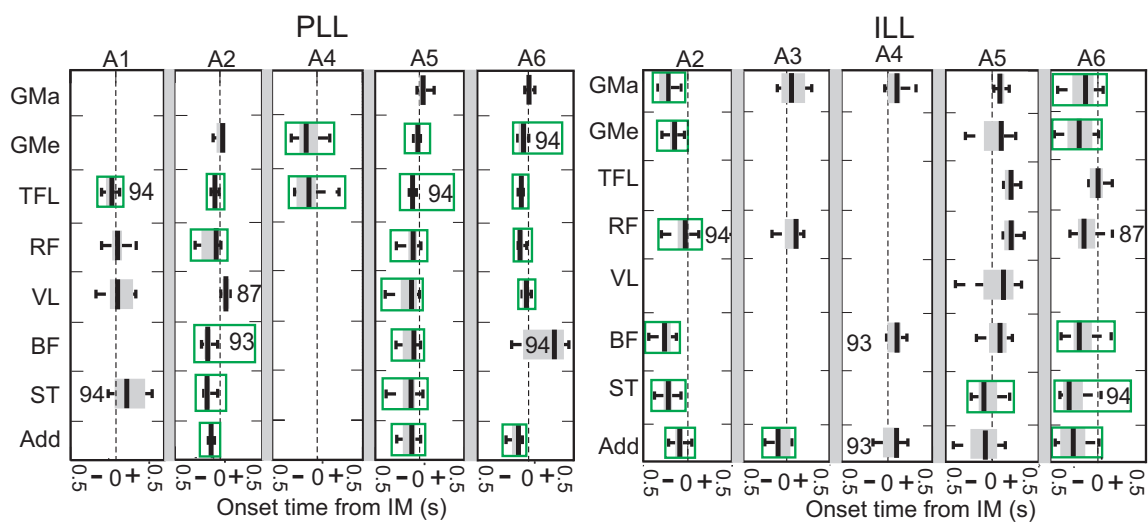

Figure 6.3: EMG onset detections with respect to IM, as determined by the inertial sensors, for the PLL (left) and ILL (right) condition. The O-lines indicate IM. Boxes placed on the left side of the 0-line, mark detections before IM (-) and on the right hand side they occur after $I M(+)$. Boxes marked in green are those detections per muscle per subject, where the median of the detections lies before IM. If a number is presented at the box this represents the $\%$ of trials included, if no number is presented all trials of this subject were included. If no box is present, the median detection is either after IM or not enough trials could be included in the EMG detection. In each boxplot the solid line represents the median, the box shows the 25 and 75 percentiles and the whiskers mark the complete range. 


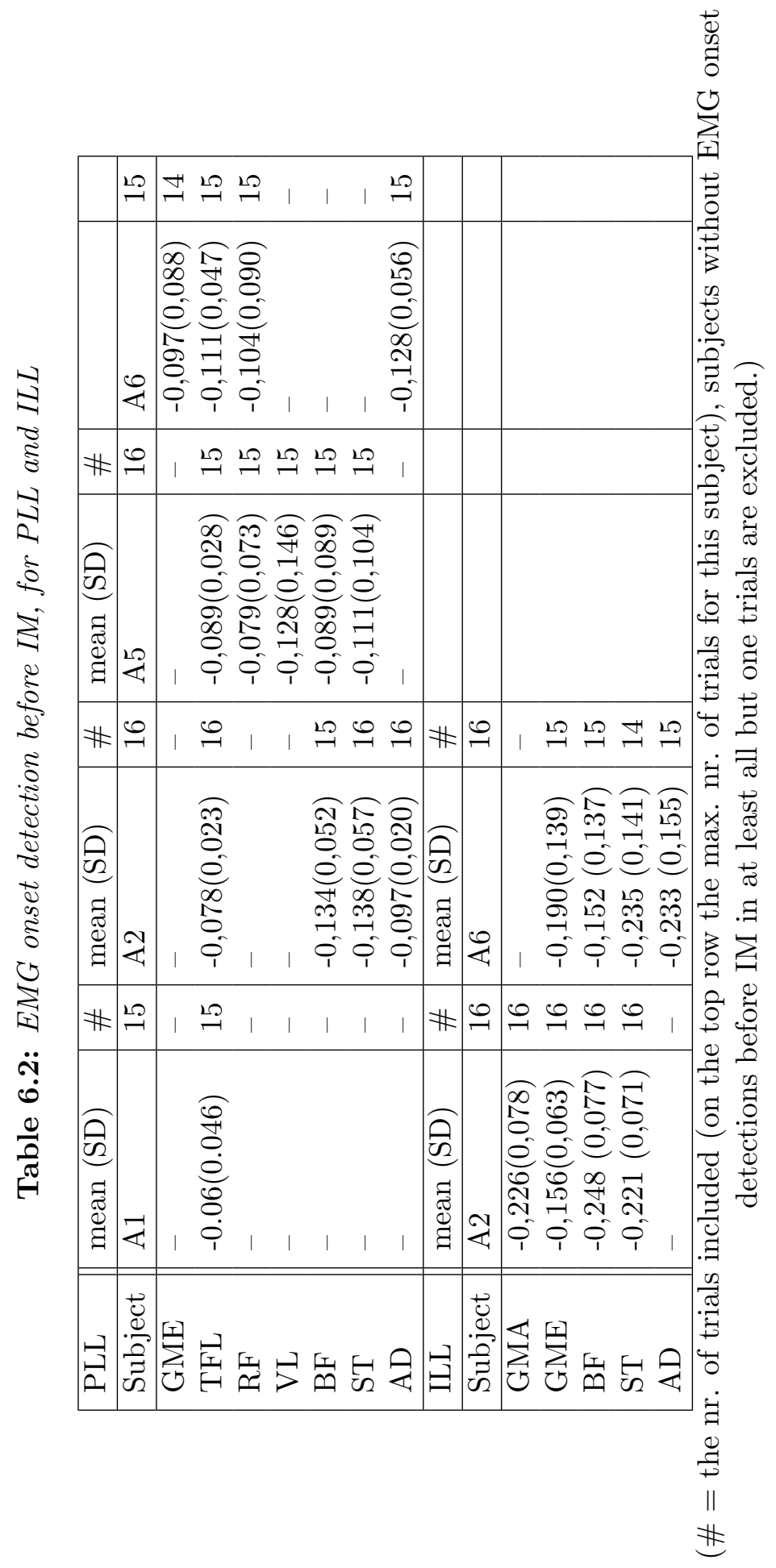




\subsection{Discussion}

The use of inertial sensors for motion detection is relatively easy and requires low computational levels and low sampling frequencies. EMG on the other hand is more difficult and requires a much higher sampling frequency, but it can show movement onset before the start of the actual movement [102]. EMG is only useful when combined with inertial sensors, for determination of motion and body position, this is necessary for correct movement onset detections. EMG can be beneficial for gait initiation detection if it has consistent and earlier detections than the inertial sensors. A faulty detection could lead to a fall and is undesirable. Therefore we only allowed one trial to be excluded per muscle per subject.

PLL - For the PLL condition, the modulus of the upper leg acc-data was able to correctly detect IM in all available trials and detected IM the earliest and with the lowest variability. The upper leg acc-data detections were up to $129 \mathrm{~ms}$ earlier than the lower leg detections. In two subjects these differences were significant. Transfemoral amputees are only able to actively control the upper leg, this is the part of the prosthesis which is moving first, followed by the lower leg. Upper leg sensors will therefore show earlier IM detections than the lower leg sensor. From the acc-data lifting of the prosthetic leg is seen, which coincides with IS. For the PLL condition IM detection is therefore similar to IS detection, even though the footswitches could not confirm this.

In four amputees one or more muscles showed onset detection between 63 an d $138 \mathrm{~ms}$ prior to IM in the required number of trials. In all four amputees TFL-EMG was a predictor of IM, with comparable variability to the inertial sensors. The only subject that did not show any EMGonset in all trials before IM walked with a waking aid, which may have led to a later muscle onset.

$I L L$ - For the ILL condition, the upper leg gyro-data appeared to be superior to the acc-data in detecting IM for the ILL condition. This was the only detection method where all trials could be included. IM was detected from the initial forward movement, which is initiated before IS. Therefore the IM detection may not necessarily coincide with IS of the leading leg. In four subjects IS was detected at the same time or earlier than IM. In one subject IM was later (90ms) than IS, but with high variation.

In two amputees four muscles were found that could predict IM up to 248 
ms in advance. In one amputee the consistency in the EMG detections was comparable to the inertial sensors detections. The other amputee showed a variability of up to $155 \mathrm{~ms}$, which will make exact timing of prosthetic control difficult.

Prediction of gait initiation using EMG may be beneficial in the PLL condition. Amputees may benefit from this, as around $70 \%$ initiates gait with the prosthetic leg [25]. When the prosthetic leg initiates gait, the knee needs to flex first and at IC of the prosthetic leg it needs to be fully extended. The prediction of gait initiation can be used to prepare the knee for flexion. Timing is essential, initiating flexion too early might lead to a knee collapse.

For the ILL condition the prosthesis should either ensure a locked knee when the leading leg goes into IS, or in future an actuated ankle could generate push-off after IS. For the ILL condition, detection of IM of the leading leg using inertial sensors will leave sufficient time for pushoff control, but it may be late to ensure a knee-lock. However, subjects already stand on their prothesis, therefore controlling knee-lock before gait initiation with the intact leg may not be of additional value and neither will EMG. In non-amputees push-off starts, approximately $300 \mathrm{~ms}$ after IS of the leading leg [102]. This suggests that even if IM is detected later than IS it would still leave enough time for prosthetic control.

\subsubsection{Methodical considerations}

Footswitch data can detect IC of the prosthetic or intact leg due to the high impact of the initial contact, but are unsuitable for IS detections in the PLL condition. This is most likely caused by the weight balance of the amputees. When standing still amputees tend to place their weight more above the intact leg than above the prothetic leg, this will not trigger the footswitches in the prosthetic leg [102].

For the ILL condition IS also remained undetected in many trials using the footswitches. This may have been caused by the weight placement of the amputee or the placement of the footswitches. More weight is often placed at the heels which may leave the toe-switches inactivated. The amputee where the detection rate was the lowest for ILL (A3) was the amputee using a walking aid, which may have caused the reduction in detections.

Although all detection methods are suitable for online detection, there is a need for a real-time decision algorithm. The EMG onset de- 
tector had a total time delay of $40 \mathrm{~ms}$, which in the PLL condition still leaves enough time to control the knee. Both detectors of the inertial sensors started with a $100 \mathrm{~ms}$ of relative rest condition, to determine if a subject is standing still. This will not lead to extra time delays due to detection, provided the subject is actually standing still before gait initiation. Changes in the gait pattern or changing from a different activity than stance to gait are not taken into account, other activities also need to be investigated. The specificity and sensitivity of the real-time detections also need further investigation. Especially for EMG detections this may be important, as in most EMG detections in the current study one trial was left out, which will reduce the specificity and sensitivity. Rather than using footswitches, a force-plate or a force sensor inside the prosthesis may provide a better estimation of the actual IS of the leading leg. Motion analysis may however still be faster, as the motion starts before the leg is lifted.

In this study the feasibility of using EMG and inertial sensors for gait initiation detection was determined using only a limited number of amputees. The variety in the amputee group was also large, which may have effected the results. The ILL condition showed differences between the amputees. This may be because of the variety in the group or the low number of subjects, but also because they may not be used to initiating gait with the intact limb. However, results from the PLL condition suggest that similar results can be found in all amputees, one subject might also be included after he received additional training. This suggests that the variety and low number of subjects did not effect the outcome for the PLL condition.

\subsection{Conclusions}

A new method is proposed to accurately detect IM from inertial sensors at the upper prosthetic leg, in both the PLL and ILL condition. For detection of IM in the PLL condition, the modulus of the upper leg accelerometer data performed best, for the ILL condition the modulus of the upper leg gyroscope data performed best. From sensors at the upper leg for both conditions, more trials could be included and in some subjects IM detections were significantly earlier. In four amputees onset of the TFL in the PLL condition was up to $111 \mathrm{~ms}$ earlier than IM detections. For the ILL condition EMG provides no additional value. Using inertial sensors (at the upper leg) for gait initiation detection 
can be of additional value to prosthetic control in both leading limb conditions, the usability of EMG seems limited. 


\section{Part III}

\section{Feedback in upper leg prostheses}





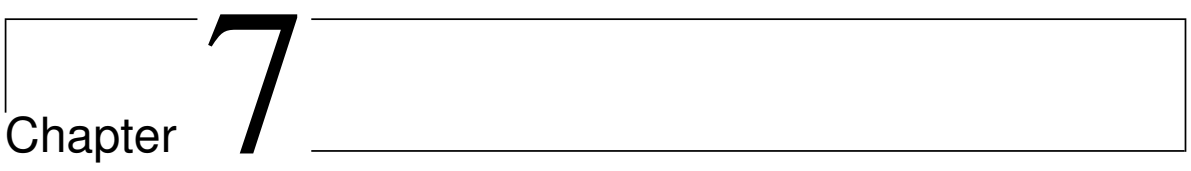

\section{Feedback in upper leg prostheses}

Feedback in upper leg prostheses may provide the amputee with valuable information about the state of the prosthesis. In this chapter we describe four short studies that looked into auditive, vibrotactile and electrotactile feedback at the upper leg. In the first study vibrotactile feedback was applied to the upper leg at different locations and subsequently in an array. Results from this showed that the posterior and medial side of the upper leg are more sensitive to vibration and interpretation of an array is difficult. In the second study vibrotactile feedback was applied with and without a socket. When providing space for the vibrator to vibrate the socket did not alter the perception, but it did increase the reaction time. In the next study electrotactile stimulation in an array was applied. Results from this study also showed difficulties in interpretation of the array and the presented information. In the last study errorbased feedback was provided during disturbed walking on a simulation prosthesis. Using auditive feedback responses to disturbances were up to $40 \mathrm{~ms}$ faster, electrotactile feedback did not show any change in the response. 


\subsection{Introduction}

In chapter 1 we presented the different options of giving feedback to transfemoral amputees. The model study in chapter 2 indicated that reflexive feedback using the new concept of prosthetic knee actuation, is most likely too slow to correct for a disturbance. If the user is part of the control and feedback loop the time delay will be too large to recover from a knee buckle. However feedback can still be useful, since it may provide additional sensory information about the prosthesis and its interaction with the environment that is otherwise not adequately observable. This may result in more confidence in ambulation and may reduce the chance of falling $[7,8]$.

This chapter provides more insight in the usability of vibrotactile and electrotactile feedback in upper leg prostheses. Although both modalities have been used for feedback before in the upper extremities, neither of them were tested for use in upper leg prostheses. As timing for feedback in upper leg protheses is critical the results may be different than for the upper extremity.

For upper extremity prostheses force feedback and position feedback are the most important types of information to be fed-back [146]. Vibrotactile and electrotactile feedback have been used to give feedback on prosthetic hand opening and touch [57]. Feedback on hand opening was presented to the subjects using an array of electrodes or vibrators in the longitudinal and transversal direction of the forearm. Additionaly, touch feedback was presented to the subjects using one stimulator, different from that of the array. The tactile feedback modalities improved the performance, compared to non-feedback conditions. However correct hand opening was only detected in $30-50 \%$ of the trials, without visual feedback. Addition of touch feedback improved the performance, however at the cost of time. Subjects performed better with vibrotactile feedback than with electrotactile feedback. Another study by Witteveen et al. [58] showed that force and slip feedback could be provided using vibrotactile stimulation in an array or at one location using frequency modulation. Force levels were correctly estimated in up to $80 \%$ of the trials, depending on the task. Force level estimation took however 1-2 seconds per trial.

Non-obtrusive stimulation at the lower extremity for the purpose of feedback in prostheses has hardly been performed. Besides the studies 
by Vos et al. $[59,147]$ and Buma et al $[148]$ on electrotactile feedback and the study by Fan et al. [50] on haptic feedback no studies were found. Vos and Buma et al. $[59,147,148]$ provided continuous feedback on the knee angle and projected normal and disturbed gait patterns on the electrodes. Of the 90 patterns 20 were randomly disturbed and subjects had to report if the projected pattern was disturbed or correct. Although their correct detection rate was around 95\%, this still implies that $5 \%$ of all projected gait cycles was misinterpreted. On average, amputees take 6000 steps a day [149], this implies that every day around 300 steps would be misinterpreted if the correct detection rate is not improved. They stimulated at $80 \%$ of the dynamic range (stimuluslevel $=$ (discomfortthreshold-sensationthreshold) $\cdot 0.8+$ sensationthreshold). This is close to the discomfort threshold and they found that reddening of the skin was seen at these high stimulus amplitudes, which is undesirable for long-term use.

Tactile stimulation causes habituation, adaptation of the central nervous system to the stimulation. Habituation can occur within 2-5 min after continuous stimulation of the skin, recovery is seen between 2-20 min., depending on stimulus type and location of the stimulus $[150,151]$. Habituation can be reduced or postponed when using high stimulus currents or intermittent rather than continuous stimulation [147,151-154].

Although Weinstein found that the ventral side of the upper leg is one of the least sensitive body parts, it is unknown if this also holds for the lateral, medial and posterior sides [155]. Vibrotactile stimulation in an array at the upper extremity has shown to be effective for providing feedback, however correct detection rates are low and timing was not considered to be critical $[57,58]$. The (continuous) feedback method presented by Vos et al. [147] may be further optimized to increase the detection rate. Continuous feedback is however not the only method to apply feedback. Discrete feedback or feedback that represents only very specific information, may be easier to interpret. This can consist of a stimulus when the lower leg is in extension or a stimulus when something goes wrong. No studies have been found on this kind of feedback.

Timing in feedback for the lower extremity is critical. Reflexes in humans have latencies around $20-200 \mathrm{~ms}$, depending on the the type and location of the reflex $[10,156]$. When a perturbation occurs during the early swing phase subjects have regained a stable position within 300-400ms. [157] Schillings et al. [156] measured EMG during stumble 
recovery; subjects were perturbed on a treadmill in the swing phase by an obstacle on the treadmill. These measurements showed that the first reactions in the biceps femoris and rectus femoris were detected between 34 and $39 \mathrm{~ms}$ after the perturbation. Several (reflexive) response peaks in these muscles were detected from $34 \mathrm{~ms}$ to $200 \mathrm{~ms}$ after the perturbation. Voluntary EMG was measured between 150 and $200 \mathrm{~ms}$ after the perturbation [156]. From this data we cannot calculate the amount of time a subject has before a stumble would result in a fall. This also depends on the type and timing of the perturbation. A perturbation in the early swing phase allows more time for recovery than a perturbation during late or mid swing.

Feedback at the upper leg needs to be fast, interpretable with high sensitivity and specificity and detectable under different weight bearing conditions, for it to become functionally useful for application in upper leg prostheses. This requires research to determine which locations are suitable for feedback, which type of feedback is best interpretable, if feedback can be given inside the socket and which feedback modality is best suited.

This chapter provides an overview of experimental studies that were performed to determine the usability of vibrotactile and electrotactile feedback in transfemoral amputees. For the vibrotactile feedback modality first some basic experiments concerning location of the vibrator, interpretation of an array and habituation were performed (section 7.2). Subsequently it was determined if this type of feedback can be given inside a socket, despite to the nature of the vibrators to stop vibrating when under pressure (section 7.3). Electrotactile stimulation was tested in an array of electrodes for giving continuous feedback, statically and dynamically (section 7.4). In addition, error-based feedback was tested (section and 7.5). Based on the obtained experimental results, the suitability of tactile feedback for the use in upper leg prostheses will be discussed.

For all experiments subjects signed a informed consent form before commencing the experiment and ethical approval was given by the local ethics committee for all experiments. 


\subsection{Vibrotactile feedback}

1

\subsubsection{Introduction}

The studies about vibrotactile stimulation in an array at the forearm as described in section 7.1 appeared suitable for giving feedback on hand opening and grip force. For feedback to be effective for lower extremity prostheses, the user must feel and interpret the signal, in a fast and adequate manner. In literature, little information is available on vibrotactile stimulation of the upper leg. In this study we therefore investigated three different aspects of vibrotactile stimulation on the upper leg and its suitability for feedback, using 8 pager motors. First we studied which frequencies are best perceived and possible locations of the stimulation, to determine which side of the upper leg is most sensitive for vibration. Secondly we studied the interpretability of an array of vibrators. Finally we investigated habituation of the upper leg to the vibration of the pager motors during continuous stimulation.

\subsubsection{Methods}

Setup

Ten healthy subjects participated in the study for experiments one and two, eight healthy subjects for experiment three. For the experiments an 8-channel vibrator was used consisting of 8 small pager motors (Fig. 7.1a), which can be individually controlled using a DA-converter by National Instruments. The pager motors are $1 \mathrm{~cm}$ in diameter and have a frequency range of 30 to $80 \mathrm{~Hz}$, frequency and amplitude are coupled. A 3D accelerometer placed on one of the pager motors was used to determine the frequency. During the experiments subjects were seated comfortably.

\section{Experiment 1}

Goal of the first experiment was to determine differences in perception of vibration at different frequencies and at different locations of the upper leg. Eight vibrators were placed at the upper leg of the subjects (Fig. 7.1b). The vibrators were placed in four pairs, about $20 \mathrm{~cm}$ apart. One pair at the lateral side, one medially, one posterior and one

\footnotetext{
${ }^{1}$ Adapted from [158]
} 


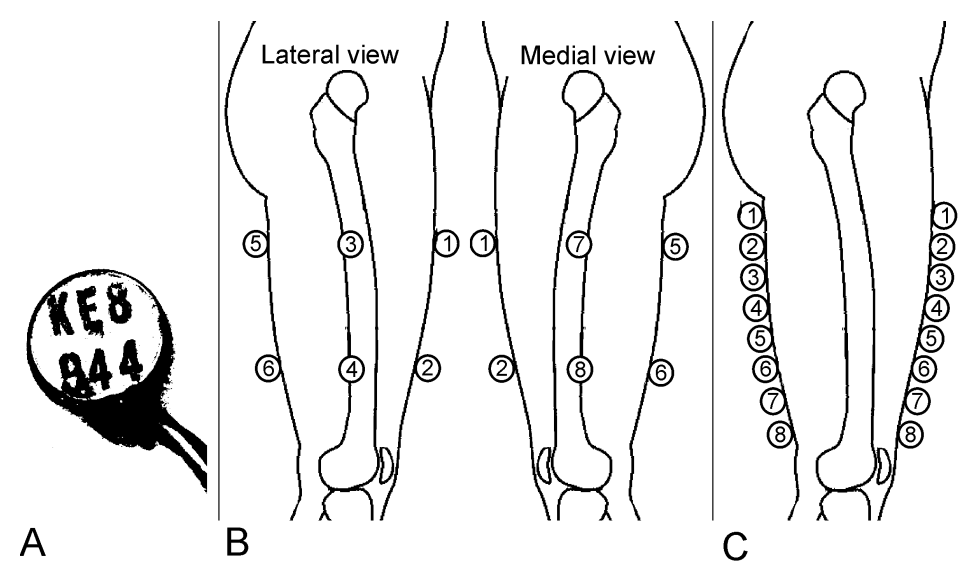

Figure 7.1: A. A pager motor as used in the experiments, by J.F. Machinery Co, Ltd., B. locations of the vibrators for experiment 1, C. locations of the vibrators for experiment 2

anterior, to cover the whole area of the upper leg. We chose to apply six frequencies in the frequency range $30-80 \mathrm{~Hz}(10 \mathrm{~Hz}$ increment) randomly to each vibrator, whereby each frequency was presented three times at each vibrator. Smaller increments were unlikely to be felt [159]. To rate the intensity of a stimulus a visual analog scale (VAS) was used. The VAS consisted of a $10 \mathrm{~cm}$ long horizontal line with on the left "no sensation" and on the right "strong sensation". Subjects were asked to cross the line according to how they perceived the stimulus, they received a new VAS for each stimulus.

First we determined if the perception of the vibrator pairs at the same side of the leg showed significant differences, if this was not the case they were assumed to be one location. Subsequently we determined per location, if there were differences in the perception for the different frequencies. Significant differences were determined using a Friedman test and post-hoc Wilcoxon signed rank tests $(\alpha=0.05)$ with Bonferonni corrections.

\section{Experiment 2}

The goal of the second experiment was to determine how well the subjects can identify patterns projected on an array. This experiment was performed at the posterior and anterior side of the upper leg. The array of 8 vibrators was placed in a row, with $2 \mathrm{~cm}$ between the vibrators, the most proximal one was numbered "1" and the most distal one " 8 " 
(Fig. 7.1c). The subjects received 50 random patterns of vibrations at $80 \mathrm{~Hz}$ (max. sensation). Each pattern consisted of 3 vibrations, whereby three vibrators were active in a sequential order each for $200 \mathrm{~ms}$, from proximal to distal and in each set each vibrator could only be active once per pattern.

The subjects were required to select the vibrators which they thought were active for each set. We determined if subjects could correctly identify the location of the active vibrators in the array. From this was determined if the three different arrays showed different results. Wilcoxon-signed-rank tests were used for statistical analysis.

\section{Experiment 3}

The third experiment was performed to determine habituation when using vibrotactile stimulation, similar as by Buma et al [153]. One vibrator was placed in the middle of the anterior side of the upper leg (vibrator $\mathrm{nr} 4$, fig. 7.1c). It was vibrated continuously for $15 \mathrm{~min}$. at $80 \mathrm{~Hz}$. The subjects were asked to rate the perceived intensity of the stimulus every 40 seconds. They were given a VAS of $20 \mathrm{~cm}$, to allow more subtle differences in sensation, and were asked to rate the fist stimulus in the middle. From this the half-life time was calculated. The half-life was calculated using an exponential fit of the data; for most subjects the VAS decayed exponentially to an asymptote. The half-life time is the time between start of the stimulation and when the stimulation reached half the original VAS score, whereby the asymptote of the exponential fit was subtracted from the original VAS [153].

\subsubsection{Results}

Experiment 1

Vibrations were applied at eight different locations, two per side of the leg. No significant differences were found in the perception (VAS scores) between the two vibrators at the same side of the leg, therefore they were considered as one location. Figure 7.2 shows the results of the first experiment for all 10 subjects together for the four different locations. For the anterior and lateral side of the leg the perception did not show significant differences between the different frequencies. For both the posterior and medial side no significant differences were found between the 30,40 and $50 \mathrm{~Hz}$ frequencies and there were also no differences between the 50 and $60 \mathrm{~Hz}$, and 70 and $80 \mathrm{~Hz}$ frequencies. All other combinations did show significant differences. 

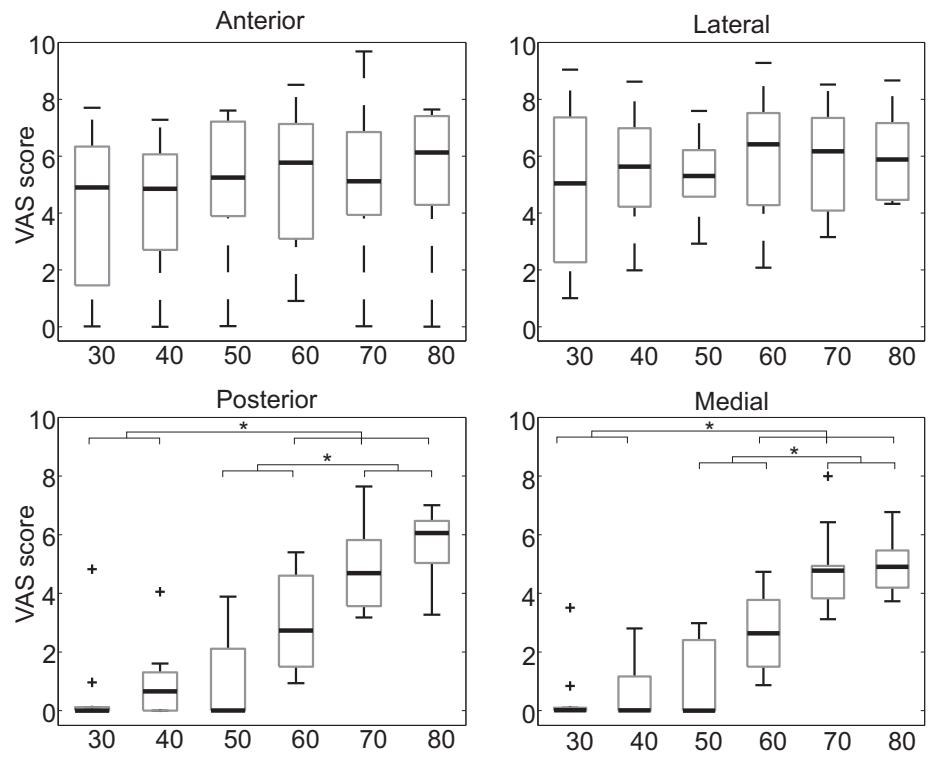

Figure 7.2: Boxplot of the VAS scores per location per frequency, combined for all subjects. Significant differences are indicated by ${ }^{*}(\alpha<$ 0.05). The solid horizontal line is the median, the edges of the boxes refer to the 25 and 75 percentiles and the whiskers extend to the extreme data points, excluding the outliers (black dots).

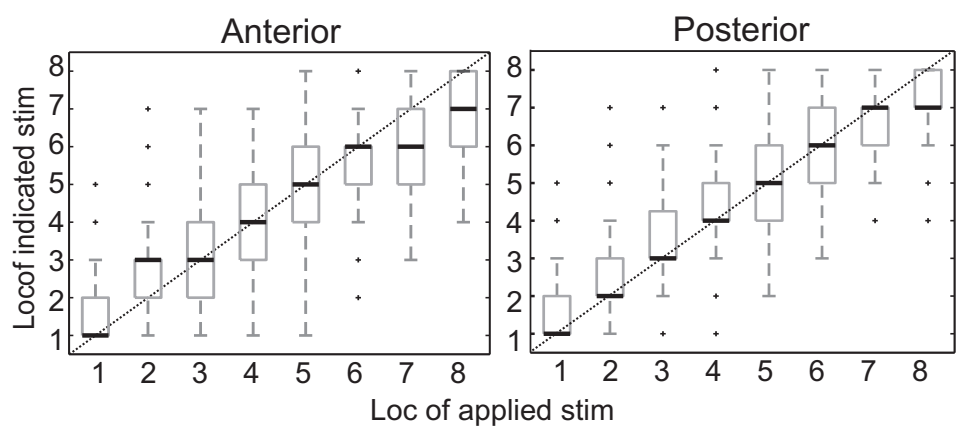

Figure 7.3: The results for the indicated location of the stimuli by the subjects as function of the applied location of the stimuli, for the posterior side (left) and the anterior side (right). The solid horizontal line is the median, the edges of the boxes refer to the 25 and 75 percentiles and the whiskers extend to the extreme data points, excluding the outliers (black dots). 


\section{Experiment 2}

Figure 7.3 shows the stimulus locations estimated by the subjects as a function of the applied stimulus locations. The diagonal line shows where the medians should lie, if the location is estimated correctly. Results from the posterior side show smaller variations in the estimated locations compared to the anterior side and in all but one location the median of the estimated location coincides with the applied stimulus location. These differences were however not significant between the anterior and posterior side. We also determined the average percentage of correctly detected patterns (all three stimuli correctly indicated) this was $3.7 \%$ for the anterior side and $7.8 \%$ for the posterior side, which is low for both and not significantly better than the guessing chance of $2 \%$.

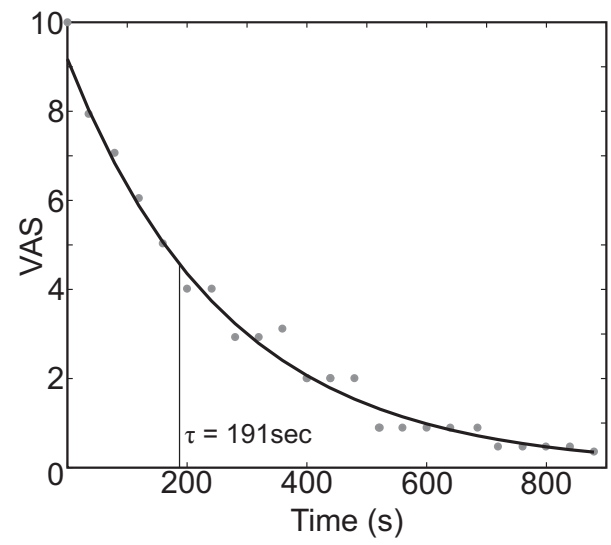

Figure 7.4: An example of the VAS score given by 1 subject. The half-life time $\tau$ of the VAS of this subject lies at 191 seconds.

\section{Experiment 3}

To estimate the time where the VAS was half the original value of 10 , a exponential fit was applied to the data. Six of the eight subjects showed an exponential decay of the VAS (see figure 7.4). The other two subjects did not show a decay in the VAS at all, the score fluctuated in both cases, but did not show a trend down or up, no half-life was calculated for these two subjects. The average half-life (SD) of the VAS in the six subjects was 290sec (150sec). In five of the six subjects the VAS was between zero and one at the end of the experiment. In one subject the VAS decayed exponential, but did not go below three. 


\subsubsection{Discussion}

The first experiment showed that at the lateral and ventral side all frequencies are perceived equally. For the posterior and medial side three frequency ranges could be distinguished, the $30-50 \mathrm{~Hz}$ range, the $60 \mathrm{~Hz}$ range and the $70-80 \mathrm{~Hz}$ range. For these two locations the higher frequencies were better perceived than the lower frequencies. This coincides with literature [10,159-161], but may also be the result of the fact that the amplitude and frequency are coupled for the pager motors; if the frequency increases, the amplitude also increases. Frequency modulation using the coin motors is not advised, as only a limited number of ranges can be distinguished, not at all locations and because amplitude and frequency are coupled.

The second experiment showed that pattern estimation in an array at the anterior and posterior side of the leg is very difficult. The number of correctly estimated patterns was around the straight guessing chance. Although the patterns did not represent a physical quantity, like the knee angle, the vibrators were active for $200 \mathrm{~ms}$ and subjects had $2 \mathrm{sec}$ to report their answer. This implies that for upper leg prostheses continuous feedback in an array will not likely be suitable.

The third experiment showed that after 290 seconds of continuous vibration the VAS reached half of its original value. This is much more than the half-life of, for instance electrotactile stimulation which is about 185 seconds for continuous stimulation [153]. However, some subjects complained after the $15 \mathrm{~min}$, that the vibrator became hot and that it would not be comfortable to keep the stimulation going on for longer. Stimulating intermittently at different locations and not continuously at the same location may resolve this. Other options are to stimulate at a lower frequency, for instance $70 \mathrm{~Hz}$, which did not show a significant lower perception, or to use different vibrators. The disadvantages of coin motors appeared to be the frequency and amplitude coupling and the low range of available frequencies, vibrators with a larger frequency range may improve the results. Advantages of the coin motors are that they are small, light weight, low in energy consumption and cheap.

From this study we can conclude that vibrotactile stimulation of the upper leg, could be suitable for feedback. Further research is however needed to determine the usability of coin motors for feedback in upper leg prostheses. The results have shown that subjects are unable to interpret 
rapidly changing dynamic patterns of vibrotactile stimulation at upper leg. Therefore it is not recommended to use continuous stimulation in patterns, but to examine the possibilities vibrotactile stimulation for feedback to give very specific information to the user. However, we did not examine the performance of subjects when providing feedback in continuous patterns, like a knee angle. Use of different vibrators may improve the results and may avoid the problem of heat development. 


\subsection{Vibrotactile feedback inside the socket}

Adapted from [162]

\subsubsection{Introduction}

Vibrotactile stimulation using coin motors has shown to be useful in giving feedback from previous studies. However the coin motors have one large disadvantage, they stop moving when the coin motor is under pressure or when the skin it needs to vibrate is too tense. Vibrotactile stimulators need room to vibrate and the skin needs room to "be" vibrated. If coin motors are to be used in upper leg prostheses, they need to vibrate inside a socket. The skin in the socket is under tension due to the full weight being placed on the socket and the residual leg is tightly fitted into the socket. Therefore we performed a feasibility study to determine if coin motors could be placed inside a socket and still be sensed by the subject, wearing the socket whilst walking.

\subsubsection{Methods}

Ten healthy subjects (non-amputees) were included for this experiment. For the experiment we used a custom made socket, which could be worn by non-amputees. During the experiments the socket was suspended from the sealing above a treadmill, such that almost the full weight of the subject was on the socket during the stance phase of walking (see figure 7.5). To place the coin motor in the socket two strips $(8 \times 22 \times 1 \mathrm{~cm})$ of PDMS (Sylgard 184, Polydimethylsiloxane) were made, with similar consistency as a normal liner. One strip had a hole of $6 \mathrm{~cm}$ in diameter and $0.9 \mathrm{~cm}$ deep, the other had a small hole just fitting the coin motor (see figure 7.6). For the larger hole the coin motor was placed in the middle of the hole on a peace of very soft padding, to allow the strip of PDMS to be placed inside the socket together with the coin motor, but still allowing the motor to vibrate. Each strip fitted tightly into the socket at the back of the leg. The back was chosen due to the better results at the back from study 7.2. For each subject the rest of the socket was filled with padding, to spread the weight evenly, as it would be in a normal socket. In all experiments 1 coin motor was used to give a stimulus placed at the center of the posterior side of the leg. The coin motor was placed in exactly the same location for each of the conditions.

Procedure

Three different conditions were randomly tested per subject. 


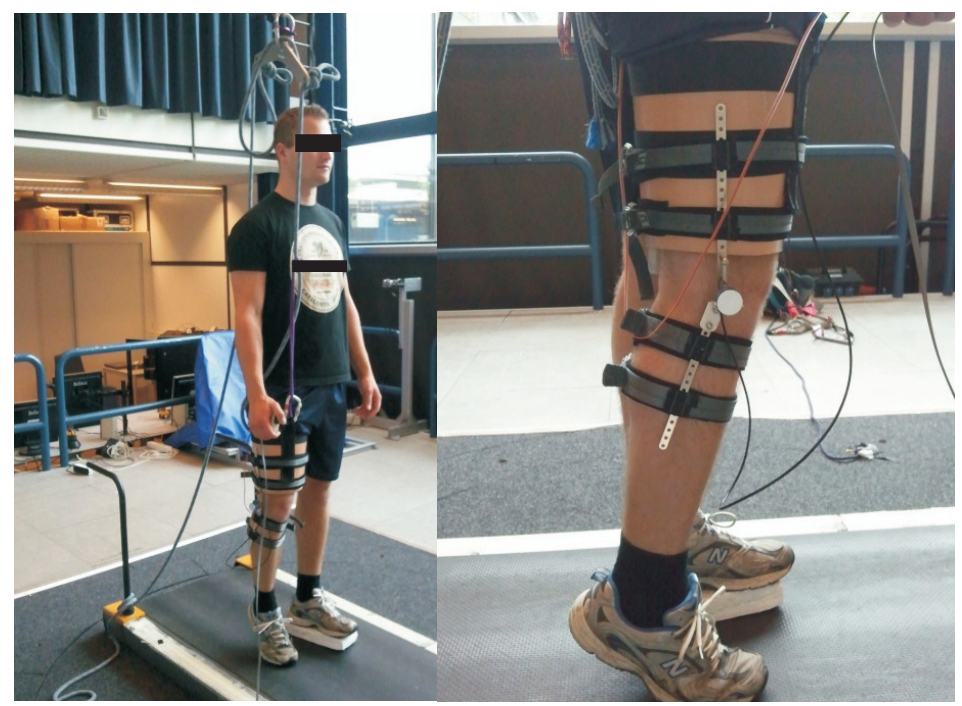

Figure 7.5: Setup of the experiment (left). The socket is suspended from the sealing above the treadmill, adjusted such that for each subject most of the weight was supported by the socket in the stance phase. The potentiometric knee angle sensor (right) is aligned with the knee joint to measure the knee angle. Under the non instrumented foot a block was placed to ease walking.

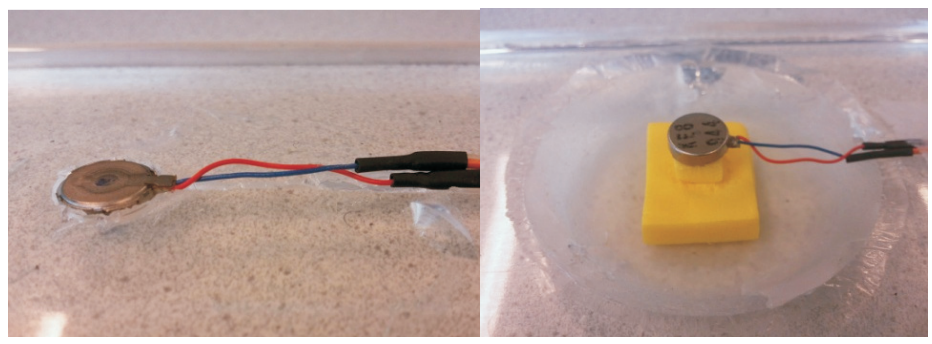

Figure 7.6: Left: the "no-hole" condition, the vibrator was placed tightly into the PDMS strip. Right: Large hole in the PDMS strip. The vibrator is placed in the middle of the hole on top of soft padding for exact placement of vibrator.

1. No socket (NS)

2. With socket, no hole (WSNH)

3. With socket, large hole (WSLH) 
Stimuli were presented to the subject in the swing and stance phase separately at a random timing, 30 stimuli each, a total of 180 stimuli per subject. Subjects had to press a button in their hand when the thought they felt a stimulus. From this data the performance for each condition and for the stance and swing phase, was determined. The performance was rated as the percentage of correctly detected stimuli. We also looked at the reaction time of the subjects per condition. A Friedman statistical test was used to determine significant differences, post-hoc Wilcoxon-signed-rank tests and Bonferonni corrections were performed where necessary $(\alpha=0.05)$.

\subsubsection{Results}

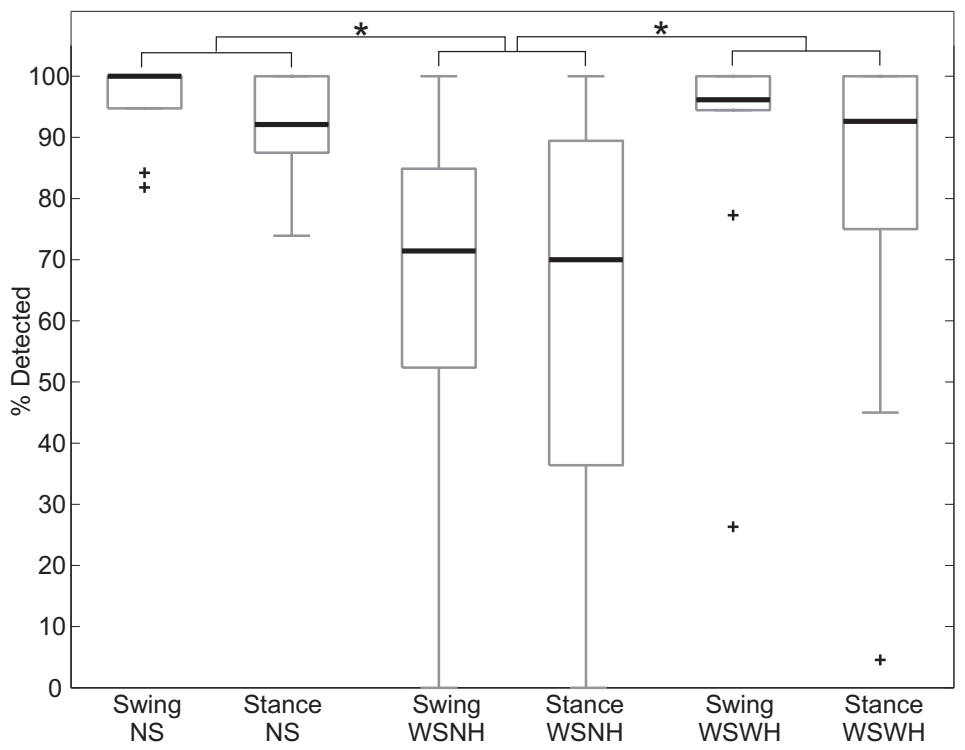

Figure 7.7: Boxplot of $\%$ detected stimuli per condition over all subjects. In each boxplot the thick line represents the median, the box shows the 25 and 75 percentiles, the whiskers mark the range outliers are indicated by $a+$. The ${ }^{*}$ indicate significant differences.

Figure 7.7 shows the percentage correctly detected stimuli for the three different conditions, and the swing and stance phase separately. Testing showed that stimulus detection scores were not statistically significantly different between stance and swing phase for all three conditions. Subsequently the conditions were tested for differences, whereby the stance and swing phase were taken together. The percentage of 


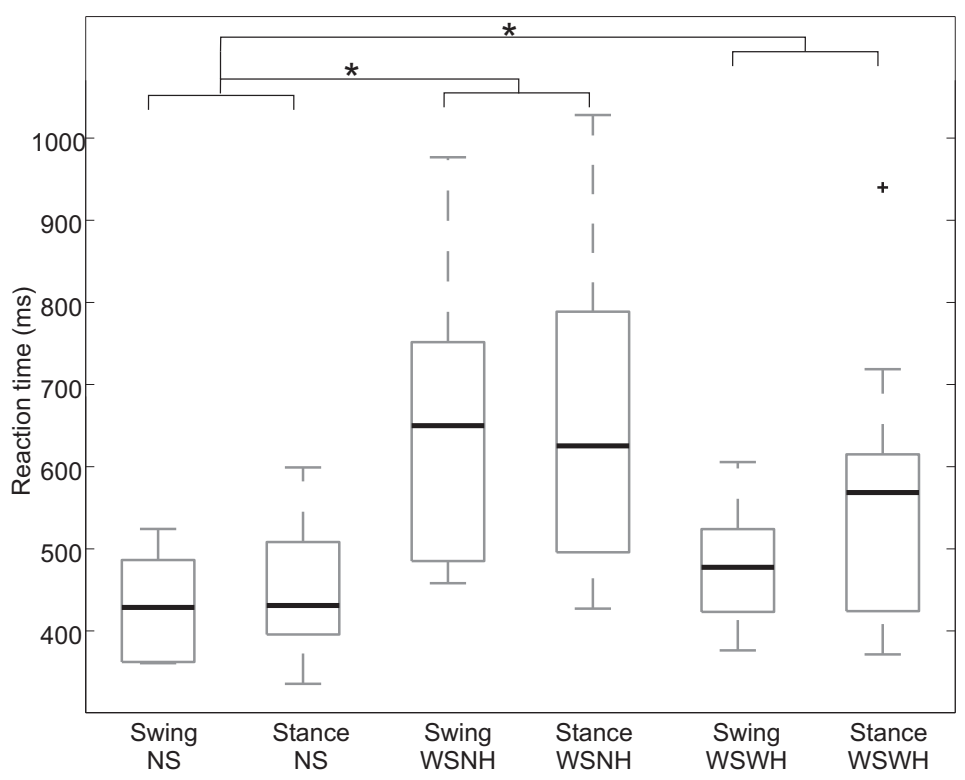

Figure 7.8: Boxplot of reaction times per condition. In each boxplot the thick line represents the median, the box shows the 25 and 75 percentiles, the whiskers mark the range outliers are indicated by $a+$. The ${ }^{*}$ indicate significant differences.

correctly identified stimuli for conditions NS and WSLH were not significantly different, but they were both significantly better compared to condition WSNH.

Figure 7.8 shows the differences for reaction time between the conditions and the stance and swing phase. Again no differences were found between the stance and swing phases within the conditions. However, both conditions with a socket had a significantly longer reaction time (both $\mathrm{p}=0.002$ ) compared to the condition without socket.

\subsubsection{Discussion}

These results show that vibrotactile feedback can be perceived inside the socket, as long as the vibrator and the skin have some space to vibrate. The WSNH condition gave significantly worse results in reaction time and the percentage of perceived stimuli.

The difference in the reaction time between NS and WSLH needs further research. The reaction time was now around $450 \mathrm{~ms}$ for the no-socket condition and $500 \mathrm{~ms}$ for the WSLH condition. Physiological reflexes in the lower limbs have a delay of around $75 \mathrm{~ms}$ between the reflexive trig- 
ger and the subsequent action [10]. Artificially triggered reflexes show EMG onset between 40 and $100 \mathrm{~ms}[77,156]$. Reaction times of visual and audio stimuli are around 160-200 ms [163]. Therefore the current reaction times may be too large to be used for feedback in lower limb prostheses.

However this reaction time may improve if feedback is given with respect to a specific condition. Now stimuli were given randomly, whereas feedback representing a specific knee angle or an error signal may be better and faster interpretable. The reaction was now also given by pressing a button, which requires a hand action whereas the stimulus is given at the leg. It is unclear if this may have effected the reaction time positively or negatively, but the effect on the reaction time would have been equally large for all conditions. Further research is needed to determine if the reaction time can be improved and how and if it will also allow enough time to react to the feedback. 


\subsection{Continuous electrotactile feedback}

Adapted from [164]

\subsubsection{Introduction}

As described in section 7.1 and chapter 1 electrotactile feedback has been used in several studies to provide feedback $[57,59,148,150,152,153]$. The study performed by Vos et al [59] showed that subjects that were given electrotactile feedback in patterns at an array of 8 electrodes placed at the upper leg could detect deviations from these patterns.

In this study our goal was to determine if the results by Vos et al. [59] could be improved by changing the position of the array on the leg. Their detection rate, the ratio between the number of correctly detected patterns and the total number of applied patterns, was $95 \%$. Changing the position of the array may leave more space between the electrodes and therefore make it easier to identify the electrodes and therefore patterns. Spreading the electrodes more evenly around the leg might make the interpretability of the array easier. Our hypothesis was that if the array of electrodes was placed not only on the anterior side, but also on the posterior side of the leg and therefore more space is left between the electrodes, the presented information would be easier to interpret. In this study we therefore examined three different arrays. For each of the arrays we performed a static and a dynamic stimulation experiment. Subjects were asked to estimate a static knee angle and to detect normal and disturbed gait patterns projected on the array. From this we determined if this continuous feedback is suitable for the use in upper leg prostheses.

\subsubsection{Method}

Setup

Seven healthy subjects (non-amputees) were included in this study. During the experiments the subjects were seated in a chair and asked to fully focus on the experiment. All stimulations were performed at the upper right leg of the subjects. During a normal gait cycle the knee angle describes a regular pattern, as can be seen in figure 7.9. Two patterns were projected on the array, one pattern had the duration of one gait cycle, whereby the knee angle during this gait cycle was made discrete by dividing the maximal knee angle by the number of electrodes in the array ( 8 or 6$)$. Each electrode in the array subsequently presented one 


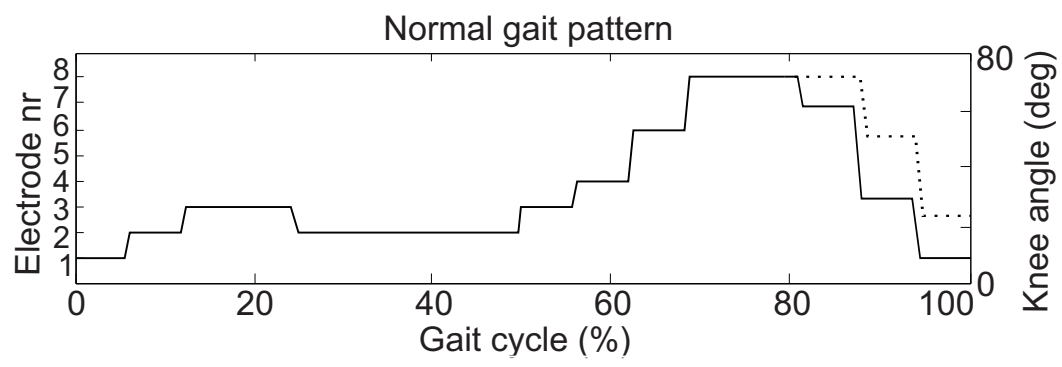

Figure 7.9: The normal knee angle pattern was divided by the number of electrodes and each electrode represents part of the range of the described pattern. During each normal projected gait cycle pattern (solid line) each electrode would be active at least once. For the disturbed pattern (dashed line) the knee remains longer in flexion, and does not reach full knee extension.

range of the knee angle pattern, an example of which can be seen in figure 7.9. The most proximal electrode of the anteriorly placed electrodes presented the "fully extended leg" in all three arrays, and the most proximal electrode at the posterior side presented the maximally flexed leg (figure 7.11). During the disturbed pattern some electrodes were active for a longer period of time (prolonged knee flexion) and the first two electrodes indicating knee extension (and almost knee extension) were not activated at all at the end of the pattern (see figure 7.9 dotted line). This disturbance could lead to a fall if there is no reaction from the user.

Sensation thresholds

Before the start of the experiments the sensation threshold per electrode was determined. This was done using the method described by Steenbergen et al. [165]. The stimulus amplitude was increased from zero in steps of $2 \mathrm{~mA}$ until the subject reported feeling the stimulus. Than the stimulus amplitude was lowered in steps of $0.5 \mathrm{~mA}$ until the subject stopped feeling the stimulus, after which it was increased again in steps of $0.1 \mathrm{~mA}$ until it was felt again; the sensation threshold. The stimulus amplitude per electrode during the experiments was 1.5 times the sensation threshold. Stimuli were square cathodal current pulses with a pulse width of $120 \mathrm{~ms}$. 


\section{Experiment 1 (angle estimation)}

In the first experiment each electrode presented one specific angle range of the knee. Subjects were trained in estimating the angle belonging to each of the electrodes. First the angle was shown on the screen and subsequently the stimulus was given at the corresponding electrode. This was first done in order of angle value and subsequently randomized, subjects had to report the angle on a visual analog scale (VAS) as seen in figure 7.10 . After subjects felt comfortable in recognizing the angle, the experiment was performed. Per electrode 15 stimuli were presented in a random order. From this data it was determined how well subjects can distinguish the electrodes by pointing out the angle, for each of the three arrays.

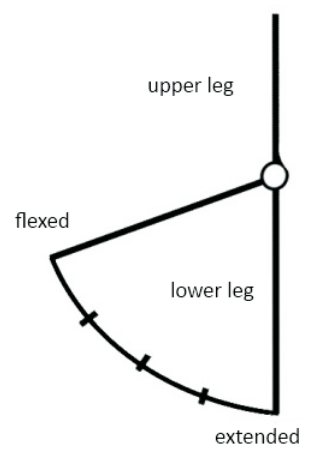

Figure 7.10: The VAS that subjects saw for rating the location of the knee angle, depending on the electrode that was stimulated.

\section{Experiment 2 (pattern detection)}

Subjects were trained in detecting the normal and the disturbed walking pattern from the electrode array. After subjects felt comfortable in the detection of the patterns the experiment was started. In the experiment the subjects were presented with 90 normal walking patterns, which were presented as if walking with a walking speed of $0.83 \mathrm{~m} / \mathrm{s}$, of which 10 patterns were randomly disturbed. The subjects had to press a button on a screen when they felt a pattern was disturbed. From this data we determined the number of true positives (TP), true negatives (TN), false negatives $(\mathrm{FN})$, false positives $(\mathrm{FP})$ and: sensitivity $=\frac{T P}{T P+F N}$, specificity $=\frac{T N}{F P+T N}$, to determine how well subjects performed. Three different arrays were tested per experiment and per subject in a random 
order, which are presented in figure 7.11.

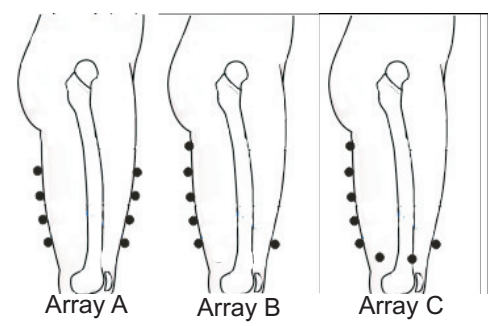

Figure 7.11: The three arrays tested in experiment 2, array $A, B$ and C.

\subsubsection{Results}

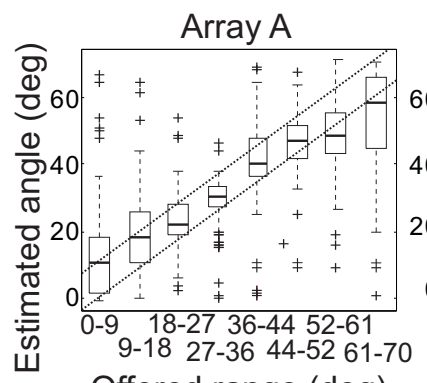

Offered range (deg)

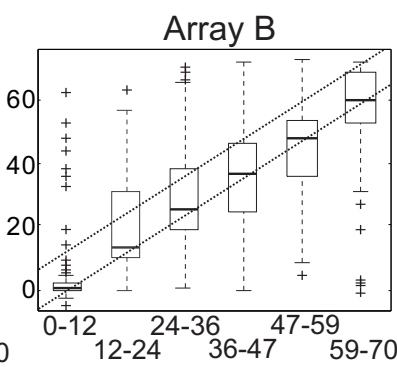

Offered range (deg)

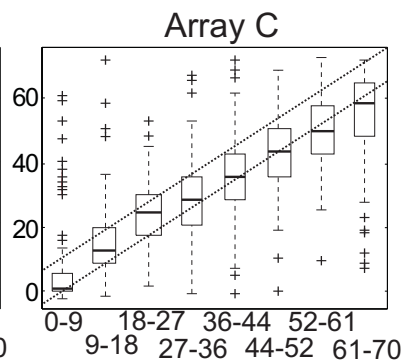

Offered range $(\mathrm{deg})$

Figure 7.12: The estimated angle by all subjects as a function of the offered angle, for the three different arrays. The diagonal dashed lines, give the range of the presented angle. For all angles to be estimated correctly, the boxplot should fall within these lines. The median is presented by the thick black line, the box represents the 25th and 75th percentiles, the whiskers give the range, the crosses give the outliers.

\section{Experiment 1}

The distribution of the estimated angle as a function of the presented angle ranges, are presented in figure 7.12. From these results can be seen that there is a relation between the presented angle range and the estimated angle, the median of each of the estimated angles generally lies within the presented angle range (between the diagonal lines). However the variation is large, some estimations are at completely the other side of the presented range. This means that stimuli presented at the front of the leg are sometimes felt at the other side of the leg. 

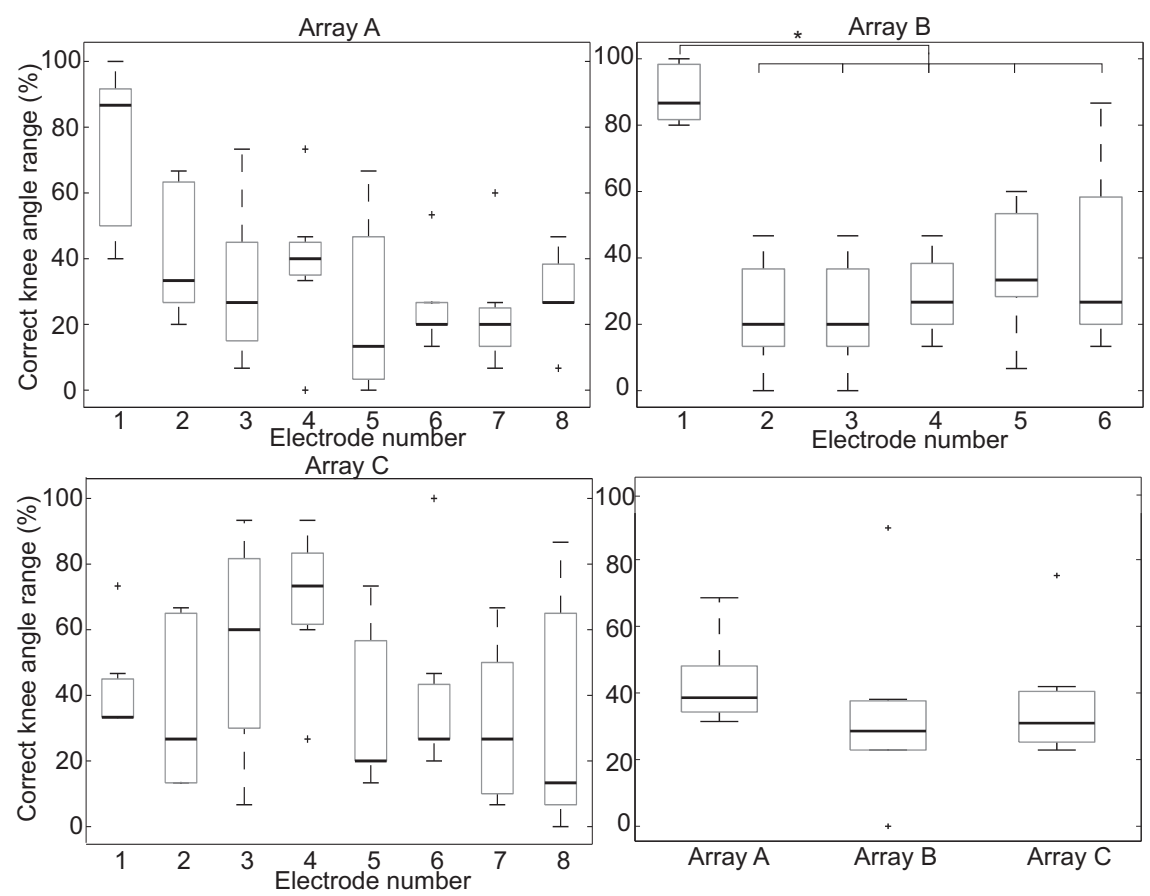

Figure 7.13: The percentage correctly estimated stimuli per electrode, for the three different arrays and the correct detections per array for all subjects. The median is presented by the thick black line, the box represents the 25th and 75th percentiles, the whiskers give the range, the crosses give the outliers.

Figure 7.13 shows the percentage of correctly detected angle ranges per electrode for each of the arrays and per array. Electrode 1 from array B is significantly better detected than the other electrodes in that array. It was also the only electrode placed at the anterior side of the leg in that array. No significant differences in detection performance were found between the arrays. For array $\mathrm{B}$ and $\mathrm{C}$ around one third of the stimuli were indicated in the correct range, for array A this was a little higher $(40 \%)$ but this difference was not statistically significant.

\section{Experiment 2}

Results are presented in figure 7.14, which shows that of the 10 presented disturbed gait patterns only 5 are on average correctly detected, which means that another 5 remain undetected and the number of FP indicates that also on average 5 correct patterns are misinterpreted. This leads to a sensitivity of around 0.45 and a specificity of around 0.75 . No 


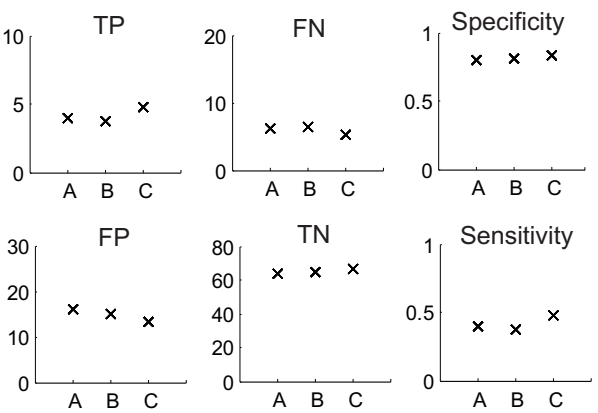

Figure 7.14: Results from experiment 2. True positives (TP), true negatives (TN), false negatives (FN), false positives (FP), sensitivity and specificity.

significant differences were found between the arrays.

\subsubsection{Discussion}

The results from the first experiment showed that an array of 6-8 electrotactile stimulators can be used to project knee angle patterns on the upper leg. However, the usability seems limited. It showed that the estimated angle is in most cases within the range of the projected angle. However stimuli presented at the front are in some cases felt at the back and visa versa. In practice this would mean someone not feeling the difference between "my leg is fully extended, I can stand on it" or "my leg is now fully flexed".

There was no significant difference between the arrays, which suggests that the location of the array does not influence the detection performance. Array B had however one electrode at the anterior side of the leg, which had a detection performance of $80-100 \%$. This implies that if feedback is presented at one specific location, it might be easier to interpret than if it is projected on an array.

The angles were not related to a physical knee angle and presented randomly to the subjects. This may have made the experiment more difficult for the subjects. If the feedback had represented an actual knee angle the stimuli would not have been completely random. The projected knee angle would always have to pass through the whole range of electrodes to indicated that the knee has moved from fully extended to fully flexed. In this experiment, this was not the case and in case of doubt the subject would just have to guess the angle randomly. If the stimulus would physically present the knee angle of a prosthesis, other 
inputs from the prosthesis, i.e. the haptic feedback, the position the upper leg and amount weight placed on the prosthesis, may give more information to better interpret the continuous feedback.

In the second experiment disturbed patterns had to be detected. On average only half of the disturbed patterns were detected and around 5 out of the 90 patterns were incorrectly interpreted. Only $45 \%$ of all disturbed patterns was detected and $20 \%$ of all steps was misinterpreted. Results by Vos et al. [147] showed higher detection ratios, although setup was similar. However in their experiments $20 \%$ of the trials was disturbed, in our experiments this was $11 \%$, which makes it harder to guess the right answer in case of doubt. In normal walking however the number of disturbed steps will be lower than $11 \%$ which will probably only make it harder to detect a disturbance. Although training and walking on an actual prosthesis may improve the results, it is doubtful if the results will be good enough to be used in an upper leg prosthesis. The subjects did not walk on a prosthesis and they did not get the haptic feedback they might have if they were walking on a prosthesis, but they also had nothing else to do but to focus on the experiment. For feedback to be used in a prosthesis it needs to be an addition, which makes it easier for the amputee to walk without thinking about it. These results do not show sufficient evidence that continuous electrotactile feedback can successfully be used in a prosthesis. 


\subsection{Error-based electrotactile feedback}

Adapted from [166]

\subsubsection{Introduction}

The previous study, in section 7.4, showed that the sensitivity/specificity of continuous electrotactile feedback is not good enough to be of additional value in upper leg prostheses. In this study we investigate a different approach of giving feedback; error-based feedback. This implies, only giving feedback when an undesired event occurs. We hypothesized that if a subject only receives feedback in case of an undesired event they will react more quickly that without feedback. In addition we evaluated the sensation and discomfort thresholds in walking and stance before and after the feedback experiments. The outcome of both experiments were used to determine the usability of electrotactile error-based feedback in upper leg prostheses.

\subsubsection{Methods}

Setup

Nine healthy subjects participated in the study. Two experiments were performed, a stimulation threshold determination experiment (1) and a feedback experiment (2). During the experiments the subjects walked with a prosthetic simulator (see figure 7.15 for the general setup).

\section{Experiment 1:}

This experiment aimed at determining the sensation and discomfort threshold per subject. Differences between walking and standing in the sensation/discomfort threshold, before and after experiment 2 were evaluated. Both thresholds were determined using the 4-up/1-down method $[167,168]$ and were determined during quiet stance and walking. Garcia-Perez et al. [167] recommended an up/down step size of $0.28 \mathrm{~mA}$. The procedure for the sensation threshold was as follows: the stimulus amplitude started at $2.5 \mathrm{~mA}$ which was always felt by the subjects, but not uncomfortable. Subsequently, the stimulus amplitude was lowered in steps of $0.18 \mathrm{~mA}$ until it was not felt anymore. At this stage the actual threshold determination started, the stimulus level was increased by $0.26 \mathrm{~mA}$ until it was felt again, it was subsequently lowered by $0.07 \mathrm{~mA}$ until not felt anymore, and than increased again by $0.26 \mathrm{~mA}$ etc until 20 stimuli were given after the start of the threshold determination. This was repeated at least twice. If the first and second threshold estimates 
were more than $0.1 \mathrm{~mA}$ apart, it was repeated once more. The sensation threshold was determined using a logistic regression curve, whereby the threshold for $50 \%$ detection lies halfway the regression curve. [167] For the discomfort threshold the stimulus level started around $4 \mathrm{~mA}$ and was increased in steps of $0.36 \mathrm{~mA}$ until the stimulation became uncomfortable. It was subsequently decreased with $0.26 \mathrm{~mA}$, until it was no longer uncomfortable, than increased with 0.07 until uncomfortable, and decreased again, etc. until 20 stimuli were given after the first uncomfortable stimulus. This was not repeated. Subjects had difficulties to determine the threshold and it gave them discomfort. The threshold was estimated by averaging the stimulus levels of the 20 stimuli after the first uncomfortable stimulus. A logistic regression was in most cases not possible.

The sensation and discomfort thresholds were determined during quite stance and during walking on the treadmill both with the prosthetic simulator, this was repeated after experiment 2. Significant differences between walking and standing and before and after experiment 2 , were tested using the Friedman test and the Wilcoxon-signed-rank test $(\alpha$ $=0.05$ ).

\section{Experiment 2:}

The setup of the measurements is depicted in figure 7.15. Subjects were asked to walk on a treadmill with a prosthetic simulator. The prosthetic simulator was equipped with the single axis knee 3R90 by Otto Bock. The lower leg of the prosthetic device was attached with a string to a device called Tripper. This device allowed a normal walking pattern using the prosthetic device, but was able to block the string at a chosen position of the lower leg in the gait cycle to prevent further knee extension. The position of the leg in the gait cycle was determined by a potentiometric angle sensor at the knee. The disturbance was presented to the subject in mid to late swing; after maximal knee flexion. The perturbation should be initiated, when the knee reached $95 \%$ of the maximal flexion angle when moving back to extension. Due to the 20 $\mathrm{Hz}$ sampling rate of Tripper, this may have been up to $25 \mathrm{~ms}$ late. Additionally there was a small time delay in the system, between the initiation and the actual string blockage. From the moment the initiation should start until the actual blocking of the string there was a delay of approximately $50 \mathrm{~ms}$. The actual disturbance was presented for $150 \mathrm{~ms}$. The actual disturbance therefore came at the end of the swing phase. Subjects were required to react to the disturbance, because standing on an unlocked knee at the end of the swing phase would cause a knee 
buckle. For each condition 20 disturbances were given, randomly spread over 400 steps, to allow recovery after a perturbation and to make the perturbations unpredictable.

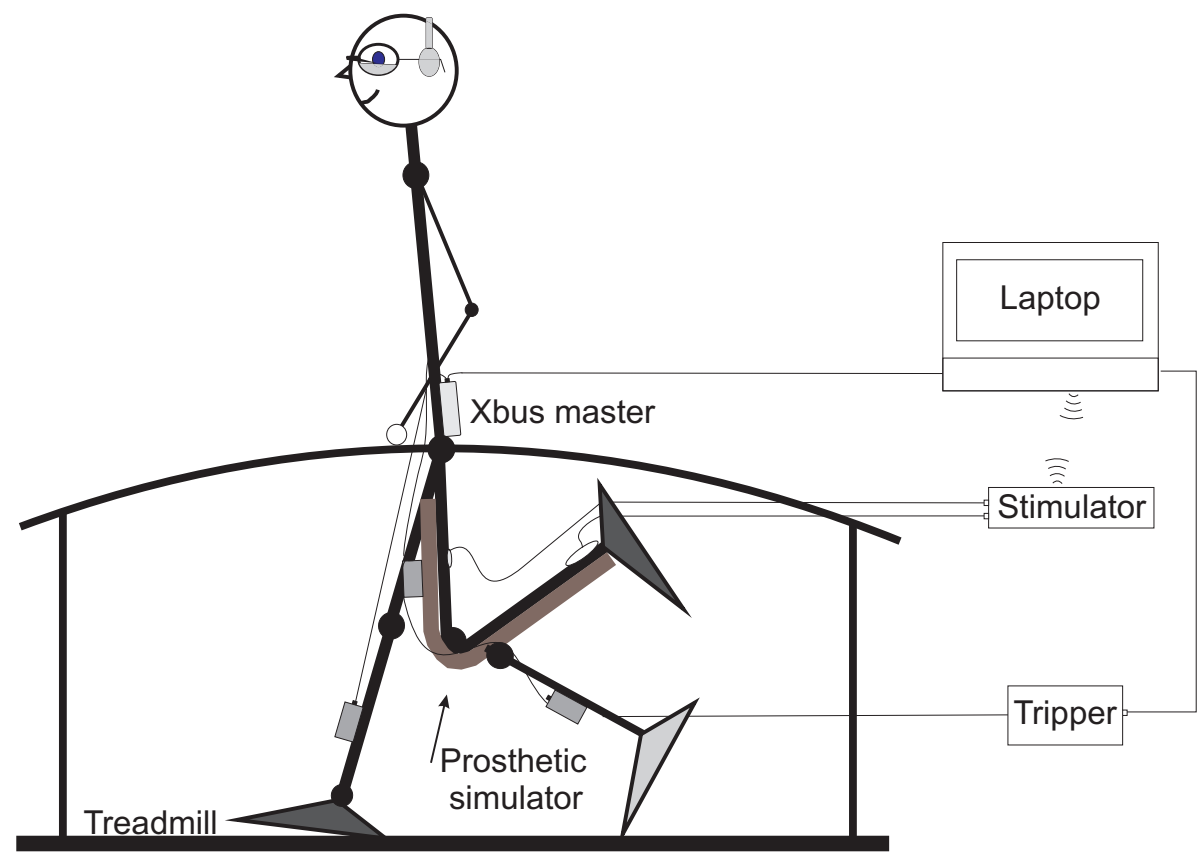

Figure 7.15: Overview of the setup of the experiment.

Four conditions were tested:

1. (nFB1) Without feedback

2. (AF) With auditory feedback

3. (EF) With electrotactile feedback

4. (nFB2) Without feedback

The order of the feedback conditions was randomized, but each experiment started and ended with the non-feedback condition, to exclude differences caused by learning. To diminish the auditory feedback in the non-feedback and electrotactile feedback conditions the subjects wore a headphone with (loud) music, but not during the auditory feedback trials. Before the experiment the subjects were trained to walk with the simulator prosthesis on the treadmill and they were familiarized with the disturbance. Subjects were protected from falling with a harness and were allowed to use handrails, but this was discouraged. Subjects 
also wore glasses that prevented vision on the prosthesis, to prevent visual feedback of the disturbance and the prosthesis.

The auditory feedback was given by Tripper itself at the time of the perturbation. The electrotactile feedback was given at the same time, at an electrode at the back of the leg, free from the prosthesis. Each stimulus consisted of 5 pulses, each pulse a square cathodal current at $80 \%$ of the dynamic range (see section 7.1 ), as determined in experiment 1.

\section{Data analysis}

Data was recorded using 3D inertial sensors by Xsens (Enschede, NL) at the upper and lower leg of the prosthetic simulator and at the lower leg of the intact limb. Subjects were only able to actively control the upper leg. Therefore the first reaction to the disturbance was expected to be seen in the upper leg inertial sensor data. From the modulus of the upper leg gyroscope data we determined the first deviation in the disturbed gait cycle compared to the non-disturbed gait cycle. The time between this first deviation and the timing of the disturbance was calculated for each of the four conditions. This first deviation in the upper leg inertial sensor data should lie at least $150 \mathrm{~ms}$ after the onset of the perturbation, because only after $150 \mathrm{~ms}$ the blocking of the string was released. If a reaction is seen before the end of the blocking of the string the subject would have had mechanical feedback of the disturbance itself. If this form of mechanical feedback exists it should be clearly visible in the gyroscope data of the upper leg, as the leg would suddenly be pulled towards extension, whilst it is moving towards flexion. Significant differences were tested using the Friedman and Wilcoxon signed rank test $(\alpha=0.05)$, with Bonferonni corrections where needed.

\subsubsection{Results}

\section{Experiment 1}

Figure 7.16 shows the results of experiment 1. Before experiment 2 the sensation threshold in stance is significantly lower than during gait. After experiment 2 this difference is no longer significant. The sensation threshold during stance before experiment 2 is significantly lower than after experiment 2 . The sensation thresholds during gait before experiment 2 does not differ significantly from the sensation threshold during gait after experiment 2, the same holds for the discomfort threshold. The sensation and discomfort thresholds are for each condition significantly different from each other. The discomfort threshold in stance after 


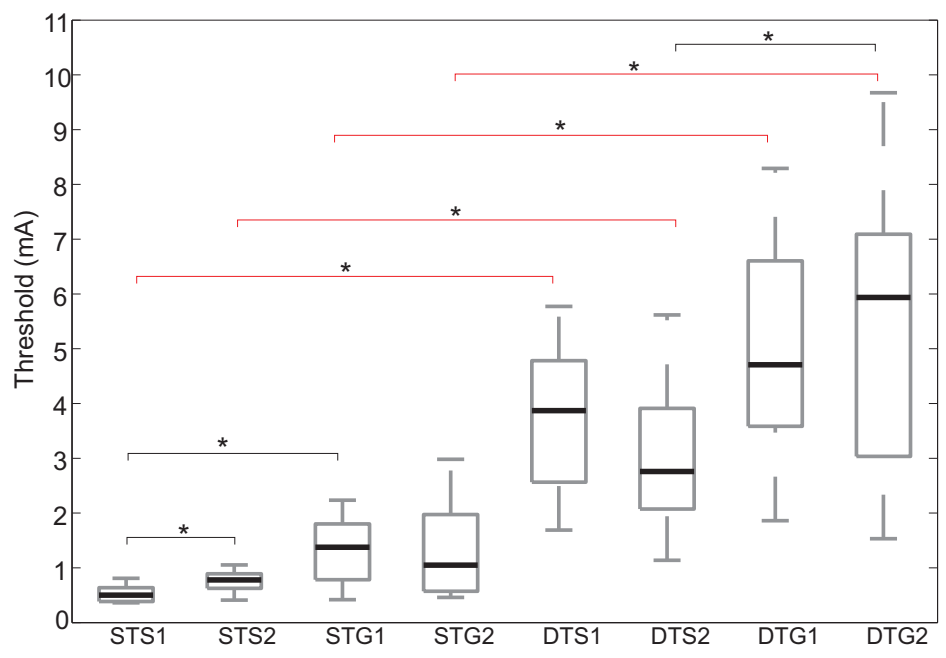

Figure 7.16: Boxplot of the results thresholds determined in experiment 1. $S T=$ sensation threshold, $D T=$ discomfort threshold, $S=$ stance, $G=$ gait, 1 = before experiment 2,2 = after experiment 2 . In each boxplot the thick line represents the median, the box shows the 25 and 75 percentiles and the whiskers mark the complete range. The * indicate significant differences.

experiment 2 is significantly lower than the discomfort threshold in gait after experiment 2 .

\section{Experiment 2}

Two "stumble" recovery strategies were found in the data, the lowering and the elevating strategy $[157,169]$. Figure 7.17 shows an example for both recovery strategies. For both strategies a change in the gyroscope pattern of the upper leg was seen. For the lowering strategy, the leg is brought down after the perturbation and initial contact of the prosthetic foot occurs on an non-extended knee, a small hop is made with the other leg. The fast lowering of the knee is represented by a peak in the gyroscope signal. For the elevating strategy this peak is lower than normal. The subject does not lower the knee, but gives the hip an extra (flexion) impulse after the perturbation which causes the knee to extend. This also shows in the elongated swing phase. (see figure 7.17) In both examples no change in the upper limb gyroscope data is seen until around $300 \mathrm{~ms}$ after the onset of the perturbation. If the perturbation would have caused mechanical feedback an abrupt change in the upper leg inertial sensor data should be visible within $150 \mathrm{~ms}$ after perturbation onset. 

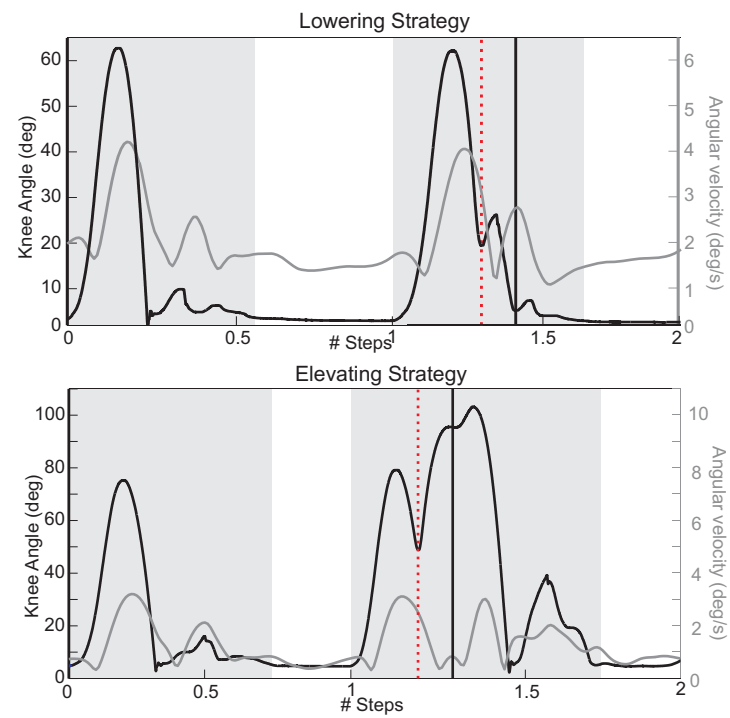

Figure 7.17: Detection examples for the lowering strategy (top) and elevating strategy (bottom). In black the knee angle, in grey the angular velocity of the upper leg. Two steps are shown for each strategy, one non-perturbed step (1) and a perturbed step (2). The shaded areas show the swing phase of the prosthetic leg, in white the stance phase. The red dotted line is the disturbance onset. The vertical black line represents the detection of the first detectable change in the upper leg gyroscope data.

For detection of the reaction of the subject for both strategies the top of the first peak in the modulus of the upper leg gyroscope data was taken as the first reaction. This is the point where for both strategies and for all subjects the first clear and detectable change in the gyroscope data was found. For all perturbations per feedback condition, the time between the perturbation onset and this peak was calculated. First we determined per feedback condition, if the two strategies had significantly different timings, which was not the case. Subsequently we determined the differences between the feedback conditions, which can be seen in figure 7.18. This shows that no differences were found between the non-feedback conditions. The only feedback condition that showed a significantly lower reaction time was audio feedback $(\mathrm{p}=0.05)$. 


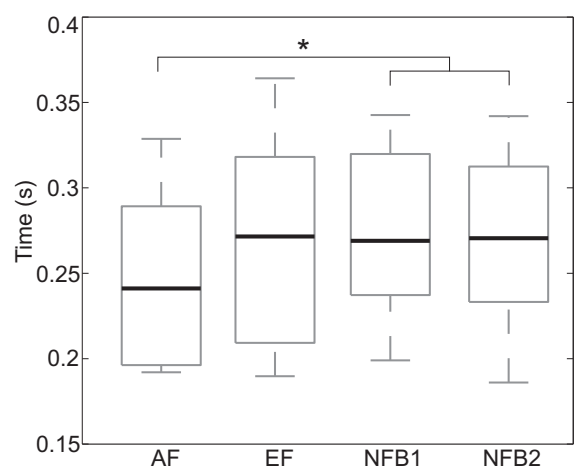

Figure 7.18: Boxplot of the first changes detected in the upper leg angular velocity, for all subjects for all four experiments (audio-feedback $(A F)$, electrotactile feedback (EF) and non-feedback (NFB1 and NFB2). In each boxplot the thick line represents the median, the box shows the 25 and 75 percentiles and the whiskers mark the complete range. The $\star$ indicates significant differences.

\subsubsection{Discussion}

Experiment 1 showed that the sensation threshold in stance is lower than during gait. This difference diminished after the second experiment, most likely due to some form of habituation. Subjects get used to the stimulus and the stimulus site may get habituated. For the sensation threshold in stance the difference between before and after experiment 2 was significant. These were also the lowest thresholds, which are most vulnerable for habituation [153]. The stimulation level during the experiment did not cause too much habituation and appeared sufficient for all experiments. This may be because of the stimulus threshold or because the stimulation was intermittent. Therefore long term intermittent electrocatile stimulation seems possible without loss of sensation. The discomfort threshold in stance is lower than during gait, however this difference was only significant after experiment 2 . This might however cause problems for the application of feedback as the stimulation threshold during gait ( $80 \%$ of the dynamic range), may become higher than the discomfort threshold during stance. This should be taken into account when applying feedback at this level. If the stimulation threshold during gait is higher than the discomfort threshold during stance, stimuli in the stance phase will become uncomfortable. Therefore the stimulation threshold may need to be lower in stance than during gait. 
Subjects reported that the discomfort thresholds were hard to determine. They found it hard to report when exactly the stimulus became uncomfortable, which causes the high variation in discomfort thresholds. Therefore only one trial was performed when determining the discomfort thresholds.

Experiment 2 showed that only audio feedback significantly reduces the reaction time by the subject. Even though electrotactile feedback was presented to the subjects at the same time as the start of the perturbation no differences were found. If this type of feedback would be implemented in a prosthesis the perturbation would first have to be detected, which would introduce an additional delay. The timing of the disturbance in this experiment coincides with a common disturbance in prosthetic walking. The disturbance leaves however little time for timely detection by the amputee. For electrotactile (error-based) feedback to become a useful addition to an upper leg prosthesis it should have shown an improvement in the reaction time in the current study, which it did not.

Although audio feedback showed a significant improvement, its usability remains questionable. A subject may not detect the feedback adequately in a crowded place or when distracted. The change in reaction time using audio feedback was around $30-40 \mathrm{~ms}$, which does not leave enough time for disturbance detection.

For all feedback experiments training was provided before the actual experiment commenced. However more extensive training may influence the results. None of the recruited subjects had prior experience in using tactile feedback. In the error-based feedback experiment subjects not only had to learn how to interpret the feedback, but also learn how to walk on the prosthetic simulator. This may have influenced the results of electrotactile feedback more than those of audio feedback, because audio feedback is more familiar. However, because no changes were found in the timing at all during the electrotactile feedback experiments it is unlikely that even after extensive training it would make enough difference for it to become useful.

The disturbances in this experiment were enough to cause an actual stumble. Subjects were however allowed to use the handrail, which prevented them from falling. Therefore the reduction in the number of stumbles was not an outcome measure. A real difference between the feedback conditions would have been a reduction in the number of stumbles. However it is unlikely that this type of feedback will be of additional value to upper leg prostheses. 


\subsection{Conclusions}

At the posterior and medial side of the upper leg higher vibration frequencies $(80 \mathrm{~Hz})$ are better perceived than lower frequencies $(30 \mathrm{~Hz})$. However frequency and amplitude were coupled, therefore it is not surprising that the higher frequencies were better perceived in the current study. Pattern recognition without a physical quantity was not successful, subjects performed no better than the straight guessing chance.

For the electrotactile experiments large variations were seen, even "completely flexed" and "completely extended" were mixed. Habituation appeared to be less in vibrotactile stimulation (290 sec) compared to electrotactile stimulation $(185 \mathrm{sec})$ [153].

Electrotactile sensation thresholds during gait before and after the feedback experiments were not significantly different. This suggest that habituation will not become a problem should it be build into a prosthesis as long as there is no continuous feedback at one electrode or vibrator, which was also found by Buma et al. [153].

The position of the array on the upper leg did not have a significant influence on the performance of the subjects. Around $50 \%$ of the disturbed patterns were missed, and of all steps around $6-10 \%$ of the patterns were mistakenly marked as disturbed. This will most likely only deteriorate when used in a prosthesis as it becomes a secondary task next to walking. Changing the electrotactile feedback from continuous to error-based did not improve the reaction time of subjects either. Audio feedback given at the same time did have a significant improvement in reaction time. Although the content of the feedback still needs further research, the study in paragraph 7.3 showed that (vibrotactile) feedback can be given inside the socket with comparable results to stimulation without socket. The reaction time however is significantly larger when it is given inside the socket, which will need further research. So far no studies were found on the maximal allowable time delay between a perturbation and a subsequent reaction, where the time delay does not lead to a fall. 


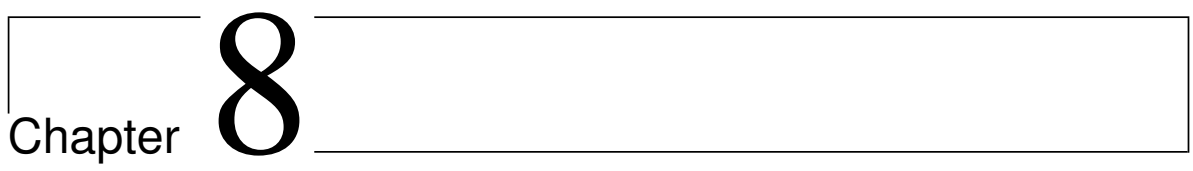

\section{General discussion}

\subsection{Introduction}

The research described in this thesis addresses the question whether a user can be part of a reflexive control and feedback loop to and from the prosthesis. Three objectives were assessed, each in a different part of the thesis:

I Asses the feasibility of the prosthetic user becoming part of the feedback and control loop of a variable stiffness actuated prosthetic knee.

II Increase the insight in kinematics and prosthetic leg EMG of transfemoral amputees and the usability of these data for voluntary control of an upper leg prosthesis.

III Develop and evaluate a method of providing interpretable feedback from the prosthesis to the transfemoral amputee, in which the amputee must benefit from the feedback when walking on the upper leg prosthesis.

Prior to this research limited information was available on the possibilities to include the prosthesis in the reflexive control and feedback loop of the user. Some previous attempts described either voluntary control of an upper leg prosthesis [40-42] or feedback designed for use inside a prosthesis $[50,59,147,148]$. In this chapter first the main results, conclusions and their implications of each of the three parts will be addressed. This discussion will be finalized with an overview of the implications of the findings for future research and (clinical) applications. 


\subsection{Reflexive variable stiffness controlled pros- thetic knee}

The newly described method in chapter 2 to control a prosthesis using variable stiffness control was predicted to be successful in correcting a small disturbance. The variable stiffness controlled knee was able to get the knee back into extension after excessive knee flexion at initial contact. Energy stored in the spring during knee flexion was re-used to extend the knee again. It was modeled such that changing the stiffness at one specific knee angle could be performed without adding or withdrawing energy from the system [61].

However, the model study also predicted that when the user becomes part of the reflexive control and feedback loop, the system becomes too slow to safely control the knee. The model assumed that an interaction by the user is only required at the occurrence of a disturbance. In the model the different steps in the reflexive control loop were modeled as one time delay (115 ms).

From parts II and III, we found that it is unlikely that this reflexive control loop will become faster. Both the feedback and control experiments showed that it is unlikely that the time delays in the model can be reduced. The preferred option would be to implement the control inside the knee, but provide additional control and feedback to the amputee. This allows active intervention when the user requires it, but also ensures a safe control loop inside the knee mechanism which acts if there is a knee buckle or other disturbance. If the amputee is also provided with feedback, trust and awareness of the prosthesis may improve. Even if effective rejection of a disturbance is not possible under user control, feedback can allow an adequate reaction of the rest of the body.

Results may however be different if the user is implemented inside the loop without variable stiffness control. If a knee is modeled which can react with small time delays to the users intent by increasing for instance the power output (like the Power Knee from Ossur) the closed loop system may be fast enough. This will however not be energy efficient, as (large) motors are required to extend the knee after a perturbation. An other issue is that this method still needs EMG detection with high sensitivity, specificity and a low detection time delays.

There are however some issues to be addressed before the method described in chapter 2 can be implemented. The model did not incorporate metabolic energy consumption by the user, from the current model 
we were therefore unable to predict if the metabolic energy consumption by the user will reduce if the proposed system is implemented, this needs further research.

In chapter 2 it was shown that changing rotational stiffness and joint moment imposed by a spring to the knee joint without changing potential energy of the spring requires energy to change the angle between the moment arm and the upper leg which is equal, but negative to the energy required to change the length of the moment arm. Currently there is no mechanism to change both without the cost of energy. An adequate mechanical solution to transfer (mechanical) energy from one spot to the other is not available yet for this specific application.

Another solution is to calculate the energy that is needed to change the arm length, and determine if this energy can be withdrawn from the spring at the cost of energy stored in the spring. Withdrawing some energy from the spring has as a consequence that the knee may not be able to get fully back into extension. However if sufficient energy is left in the spring to almost fully extend the knee after changing the arm length, this may be acceptable. It should be possible to harvest and store energy during undisturbed gait and subsequently return it to the user in the event of a disturbance. Even if we withdraw some energy from the spring we need a mechanism to change the length of the arm. This can again be fully mechanical, but no mechanism for this is available yet or we need an energy efficient method to convert the mechanical energy gained at one location into electrical energy, transfer it to another location and convert it back to mechanical energy. So far these mechanisms that convert one type of energy into the other, still need an energy supply to change the compliance [75].

\subsection{Voluntary control of a prosthetic knee}

\section{Muscle activity patterns}

In chapters 3 and 4 the kinematic and EMG patterns of transfemoral amputees during level, slope and step-by-step stair walking were compared to control subjects. In the following, we will first discuss the differences and similarities between level and slope walking in amputees compared to controls. Subsequently these two activities are compared to stepby-step stair walking. All EMG activity and kinematic data described here are from the prosthetic leg of the amputees, unless stated otherwise. 
In level walking we saw large spatio-temporal asymmetries between the intact and the prosthetic leg. The double support phase in level walking when the prosthetic leg becomes the stance leg was twice as long (20\% of the gait cycle) as in controls (10\%). Similar results were found for slope walking. Knee flexion during early stance (yielding) in amputees in level and slope walking is also reduced compared to controls. For level and slope walking knee flexion during stance was between 2 and 4 degrees for amputees, for controls this was 13 degrees for level and slope walking. The reduced knee flexion in early stance is an adaptation, because amputees are unable to allow a knee flexion moment or they are afraid of a knee buckle due to the lack of control over the knee.

Hip adduction during swing was larger in controls than in amputees for level and slope walking, but this difference was only significant in level walking. Hip abduction was similar for level and slope walking.

Hip extension is less in slope ascent and descent compared to level walking, although not significantly. The hip extensors and the adductor showed prolonged activity during the stance phase of slope ascent and descent, compared to the stance phase of level waking. For slope descent this may be explained by the tendency of the amputee to keep the knee in extension by "pulling" the hip in extension. For slope ascent, the increased muscle activity may be a method to increase forward propulsion.

In addition the lower leg muscles of the intact leg also show increased activity duration in slope walking compared to level walking during stance and swing. The reason for this is most likely that on level ground there is less need for additional adjustments from the intact leg. Besides these two differences, muscle activity patterns during level and slope walking in amputees are similar.

Although step-by-step stair ascent and descent are very different from level and slope walking, similar adaptations from the amputees were found in all three activities. Large asymmetry was visible in all three activities. In stair descent the double support phase, when the prosthetic leg becomes the stance leg, was increased compared to controls. The stance phase of the intact leg is also longer for stair ascent and descent, compared to controls.

For stair walking, hip abduction was larger in amputees, although not significantly. This increase in hip abduction is most likely an adaptation to ensure foot clearance. Step-by-step stair walking is not a continuous cyclic process, each step is negotiated from stance. Due to the absence 
of a knee flexion and extension moment and a reduction in the forward hip motion, knee flexion in step-by-step stair walking is low, especially in early swing. Amputees therefore use abduction to ensure foot clearance.

Amputees have the tendency to stand longer on their intact leg and weight is carefully placed on the prosthetic leg, causing a longer double support phase $[6,26,27]$. For all measured activities, it seemed as if the intact leg compensates for the loss of actuation in the prosthetic leg.

During rehabilitation the focus should not only be on level walking, but slope and stair negotiation are just as important. Symmetry should not be the aim of the training but efficient use of the prosthesis is more important. Although four amputees were equipped with a MPC knee, none of them used knee flexion and only two were able to walk down stairs in a step-over-step manner. Training during rehabilitation should make amputees more comfortable with the increased possibilities of a MPC knee, so amputees can fully benefit from such a knee. Training should aim to acquire the right balance between the use the intact and prosthetic leg such that both legs are used to their full extend but no harm is caused to either of the legs due to excessive use.

To prevent interference of the measurement setup to the walking patterns of the amputees as much as possible we made as little adjustments to the prosthesis as possible, to measure EMG and kinematic data. We therefore measured inside the own socket of the amputees. In most studies EMG is measured using an experimental socket. A socket that does not fit properly, due to cavities, lack of a liner, or misaligned electrodes may affect the walking pattern of the user and the EMG data. Although no direct comparison was made between experimental sockets and our data, the data did resemble that of controls.

\section{Gait initiation detection}

In the last two chapters ( 5 and 6 ) of part II, intention detection of gait initiation was examined. We looked at spatio-temporal data, kinematic data from inertial sensors and EMG of muscles of the prosthetic leg measured inside the socket. First we determined if this intention could be detected in control subjects and subsequently in amputees. The prosthetic situation was mimicked in control subjects, therefore these legs are called the mimicked prosthetic/intact leg.

The results found in the amputee group for GI were different from 
that of the controls. Footswitches did not provide adequate information on initial swing of either of the leading legs in amputees. Therefore the exact start of initial swing was difficult to determine in amputees. However, the inertial sensors at the prosthetic leg were able to detect the initial motion (IM) in both leading leg conditions.

Gait initiation (GI) detection, by means of initial swing prediction, in non-amputees was possible for both leading leg conditions. When the mimicked intact leg was leading inertial sensors could not predict GI, but EMG detection was up to 220 ms before initial swing by the gluteus maximus, gluteus medius, the biceps femoris and the tensor fasciae latae.

In amputees for the intact leg leading conditions IM was detected $70 \mathrm{~ms}$ before IC using the modulus of the upper leg gyroscope data. This initial movement was most likely the first forward motion of the amputees. Exact timings of initial swing were difficult to determine. Therefore comparing the results of the amputees to those of the controls becomes difficult for this condition.

For the intact leg leading condition only one amputee showed consistent EMG onset up to $250 \mathrm{~ms}$ before IM. Results of this amputee are consistent with those of the control subjects, three out of four muscles were similar (gluteus maximus, gluteus medius and biceps femoris).

In controls when the mimicked prosthetic leg was leading, initial swing was detected up to $260 \mathrm{~ms}$ in advance by the inertial sensors and up to $200 \mathrm{~ms}$ in advance by EMG, by the tensor faciae latae and the rectus femoris.

In amputees for the prosthetic leg leading the modulus of the 3D acceleration of the upper prosthetic leg showed movement onset around 70-90 ms before initial contact of the leading leg (IC). This initial movement roughly coincides with initial swing, because the detected motion in the accelerometer signal is the lifting of the leg.

EMG detection in amputees for the prosthetic leg leading condition, showed consistent onset detection in four out of five amputees, up to $140 \mathrm{~ms}$ before IM. In all four amputees the tensor facia latae (TFL) showed consistent detections before IM, which coincides with the results from the controls.

The detection time delay of EMG is around 20-50 ms (see chapter 5 and 6). Inertial sensors (and force sensors) are currently used in MPC prosthetic knees and lower legs, allowing them to control the damping 
of the knee with respect to the gait cycle or at the occurrence of a knee buckle $[4,5]$. Adding EMG to these microprocessors will add a considerable amount of time, because EMG needs to be detected, processed and a decision algorithm is needed (see chapter 5 and 6 ). Currently update rates of MPC knees are around $50-100 \mathrm{~Hz}$ depending on the knee and for EMG the sample rate needs to be at least $400 \mathrm{~Hz}$ for it to be used effectively $[4,5,80,100]$. For EMG to be of any use and to be of additional value to current prostheses it must be at least as consistent and at least up to $50-100 \mathrm{~ms}$ earlier than when using inertial sensors. For the prosthetic limb leading condition this would leave enough time to control the prosthesis.

Another issue with EMG is the number of false detections, because of the inherently stochastic nature of the signal. In chapter 6 we have seen that although there are consistent onset detections before IC, there are also a large number of onset detections that are unrelated to IM, IS or IC. In this thesis we did not go into the issue of the specificity of the detections. This is still a major issue, that will need further research.

Adding EMG of the TFL may be possible inside the socket of an amputee and it can improve prosthetic control for the prosthetic limb leading condition. For this condition prosthetic knee flexion could be initiated after IS and prosthetic knee extension can be ensured before IC. The diversity of the group was large but the resulting patterns were very comparable. Therefore it is assumed that main control of the prosthesis does not need to be evaluated on an individual basis, but exact timings for prosthetic control can be adjusted individually.

For the intact leg leading only one amputee showed consistent EMG onset before motion detection by the inertial sensors. It is therefore not recommended to use EMG for detection of initial swing for the intact leg leading condition. There also seems to be little use for it. If the intact leg leads the prosthesis either needs to be locked, which is already possible in current prostheses or if a powered ankle is added to the prostheses it needs to provide artificial push-off in time. There is however no need to predict initial swing of the leading leg for this purpose, because push-off of the trailing leg starts after initial swing (see also chapter 5 and 6). Using inertial sensors would therefore leave enough time to control ankle push-off.

Besides GI no further research was performed on voluntary control in this thesis. However, although the EMG data from level slope and 
stair walking showed clear patterns, the variability in the data was larger than in controls. It is unlikely that the use of EMG during normal walking for control of a prosthesis will become as reliable as the inertial sensors already used in MPC knees. Addition of kinematic sensors to the upper leg and GI detection to the prosthetic knee using these sensors may well be of additional value for both leading limb conditions. The prosthesis can be controlled for knee flexion and extension and additionally push-off in case and active ankle is added.

From the stair walking data we found that amputees have the tendency to use hip abduction to ensure foot clearance. GI detection in upper leg prostheses could allow knee flexion at the first step, to reduce the hip motion and ease foot clearance. Research on initiation detection of stair and slope walking will therefore also be of additional value.

\subsection{Feedback in upper leg prostheses}

The Reflex-leg project aimed at providing non-invasive feedback in transfemoral amputees, therefore the number of modalities for providing feedback were limited. There was only little information available on tactile feedback of the upper leg, although for the upper extremity some examples were already available $[57-59,147,148]$. Electrotactile and vibrotactile stimulation gave the most promising results for providing feedback at the upper extremity and were therefore picked to be further investigated for the lower extremity.

First we performed some basic experiments on vibrotactile stimulation of the upper leg. We found that frequency modulation with the coin motors is not suitable for stimulation at the upper leg. Although subjects were able to distinguish stimulation of multiple vibrators in an array, variation in the number of correct detections was high. Correct detection rates of all locations of three active vibrators in an array of eight were just as high as the straight guessing chance. Therefore providing continuous feedback using an array of vibrators at the upper leg does not seem useful. The information we provided however was random and not related to any physical quantity, which may have made it harder to interpret than when the knee angle or the position of the lower leg would be projected.

For both electrotactile and vibrotactile stimulation continuous feed- 
back at the upper leg did not show promising results for further use or investigation in upper leg prostheses. Discrete feedback, or error-based feedback was therefore also investigated. We designed the error-based feedback experiment (chapter 7.5) such that feedback was given at the same time as the disturbance was applied.

From the experiment we found a $30-40 \mathrm{~ms}$ lower reaction time when using audio feedback compared to using electrotactile feedback and no feedback. No differences were found between the electrotactile feedback and no feedback condition. If error-based feedback would be implemented into a prosthesis there would be the need of an additional algorithm for detection of the disturbances. This algorithm would need some time to detect the disturbance which lies in the order of 50-70 ms [76]. Therefore the $30-40 \mathrm{~ms}$ time gain found when using audio feedback will most likely not be enough for an application in prosthetics. Error-based discrete tactile feedback was therefore also considered unsuccessful for use in upper leg prostheses.

This also confirms the outcome of the model in chapter 2. In the model we already predicted that reflexive feedback, with the user in the loop, will be too slow to overcome a disturbance. In this experiment the time delays for disturbance detection (50-70ms [76]) and EMG detection (20$50 \mathrm{~ms}$, chapter 5 and 6 ) were not yet taken into account and reactions to feedback were already too slow.

In the error-based feedback experiment we did not examine if feedback had an effect on the EMG onset of relevant muscles, such as the rectus femoris or the biceps femoris. From the gyroscope data of the upper leg we did not find any improvements in reaction time using electrotactile feedback. Therefore we also do not expect that if EMG will be used to control the prosthesis that the reaction time to feedback will improve. However we only gave tactile stimuli, which does not necessarily trigger reflexes. Tactile stimuli most likely require the subject to think about the actions to take after they felt the stimulus. Even though training was provided to the subjects in all experiments, this may never become as fast as a reflex. Triggering reflexes at the lower leg has been performed previously $[77,156]$, but at the upper leg this has not been performed before and may require further research. Different disturbances may need different reactions by the amputee and therefore different reflexes to be triggered.

All feedback experiments were performed in non-amputees. Results may be different when tested in amputees. Feedback will only be of 
use if it becomes second nature or when it gives additional information when performing secondary tasks. The detection rate was already low when only focussing on detection of disturbances in a continuous signal. Haptic feedback already present in current prostheses may make additional continuous feedback easier to interpret especially in cyclic motions. Alternatively, this may become more difficult due to interpret due to many more skin sensations present and more information to process. The results from the continuous feedback experiments (chapter 7.2 and 7.4) do not suggest that detection rates will ever be high enough to be used in upper leg prostheses in a useful manner.

Some results may however improve if the applied stimuli are coupled to a physical quantity, such as the knee angle. Random patterns are difficult to interpret (see chapter 7.2). After some stimuli there is no reference to the correct position of a stimulus. In the current studies subjects were always asked to determine which knee angle they thought was projected, or which stimulators they thought were active. Once subjects loose sense of the location of the stimulation it is unlikely they will be able to correct this; they are unable to reset the reference. For the upper extremity experiments, subjects were in control of the stimulation. They were presented a hand-opening position and had to tune the stimulus to reach the desired hand-opening $[57,58]$. Currently there is no voluntary controlled upper leg prosthesis, therefore performing the experiment similar to these upper extremity experiments would not have been useful. If this feedback were applied in an upper leg prosthesis, subjects would have an extended knee every step. This may help them to reset their reference which may improve the interpretability of the feedback.

In section 7.3 we showed that using small cavities in the socket wall, releasing the pressure on the skin and vibrator, allows feedback to be transferred to the subject even inside the socket. This can become useful when a suitable method is found to provide interpretable feedback. Further research on this is however also needed. The reaction time using a socket significantly increased compared to the non-socket condition. It was also mentioned that cavities in the socket may effect the socket fitting and therefore the applicability of using this in prostheses also requires further research. The study in chapter 7.5, also showed that long term intermittent stimulation with electrotactile feedback is possible and does not significantly effect the sensation thresholds. 


\subsection{Conclusions}

From this thesis it can be concluded that it is unlikely that safe control of a prosthesis will be fast enough, if the user is made part of the reflexive loop of an upper leg prosthesis by means of non-invasive feedback. The control inside the knee as it is currently implemented in MPC knees should remain the main automated control, especially for disturbance reactions, but may be extended by voluntary control. Variable stiffness control showed to be a worth while principle that can become useful, if more research is performed on the mechanism to implement continuous variable stiffness control.

Control of a prosthetic knee can benefit from inertial sensors at the upper leg, rather than solely in the lower leg. Prosthetic control can be extended or improved using surface EMG (of the TFL) inside the socket of the prosthesis in addition to inertial sensors. It allows for early prediction of gait initiation when the prosthetic leg is leading and and may be useful for detection of other activities as well. However measuring EMG also comes with disadvantages, higher sample rates are necessary, more calculation time is required due to the inherent stochastic nature of EMG, and it will always be an addition to inertial sensors. It is therefore uncertain if voluntary control using EMG will provide faster control and if it is reliable enough to safely control a prosthesis. If the prosthesis becomes voluntary controlled it is likely that a strong need for feedback also arises.

So far we did not succeed in transferring useful information to a subject at the upper leg using electrotactile and vibrotactile feedback. However we did find that vibrotactile feedback can be used inside a socket under weight bearing conditions.

\subsection{Future research}

Future work on variable stiffness control for the use in upper leg prostheses should focus on the design of a mechanism to implement the continuous variable stiffness control. It is recommended to first model the possibilities to acquire some energy from the spring to reset both the arm and the angle. In addition, the possibilities to store energy during normal gait cycles and use this during disturbed gait cycles can be investigated. 
For feedback to become useful to transfemoral amputees it needs to become faster and better interpretable. If non-invasive methods are further explored it is recommended to perform this in patients and to make use of the haptic feedback of the socket. The final application must enhance the haptic feedback that is already provided by the socket. Electrotactile feedback was not interpretable in an array and not fast enough for error-based feedback. If the sensation of a disturbance in the walking pattern is fed-back using a painful stimulus, the subsequent reaction by the subject could be faster. This also has disadvantages; the stimulus may not be given too often and users may find it unacceptable. However a painful stimulus may also be better than a fall. First, however, it should be determined how long it will take to detect a disturbance, which disturbance it is and if feedback is required. If too much time is required to detect a disturbance the whole idea of error-based feedback should not be further investigated. This disturbance detection may however be used inside the prosthetic knee for automatic disturbance rejection.

Vibrotactile feedback can be sensed inside a socket under weight bearing conditions. Further research on vibrotactile stimulation should focus on the possibilities to provide positive feedback to amputees inside the socket about one very specific state of the prosthesis. Our research showed that feedback in an array is too difficult to interpret under time constraints, as in gait. However one array showed that an electrode separated from the rest (see chapter 7.4) is better distinguishable than the rest of the array. Electrotactile feedback was also occasionally felt under the reference electrode, which makes interpretability of an array much harder. This is not an issue in vibrotactile stimulation and for positive feedback, vibrators may therefore be very suitable. Training is an important issue in providing feedback and it is therefore also recommended to perform future experiments using experienced prosthetic walkers. Trained amputees can focus on the experiment rather than on walking itself and therefore determine much easier if the feedback is a useful addition.

A different type of feedback may be introduced through enhanced haptic feedback. The prosthesis, including controlled actuation, imposes a dynamic load on the user. The haptic feedback provided by the imposed load as experienced by the user may be modulated to provide additional relevant information covering the state of the prosthesis. The modulation of the actuator that effects the feedback should however not interfere with safe control of the knee. No studies have been found on this topic, but it may be a worthwhile principle, although more research on the feedback an amputee already receives from the socket-residual 
limb interface is also required.

The Reflex-leg project intended to introduce feedback and control to the prosthesis but in a non-invasive manner to the user. This greatly reduced the methods of applying feedback and also excluded nerve sensing and stimulation for control and feedback in prostheses. Although Clippinger at el. [49] showed that a finite number of knee angles could be fed back to the amputee by an implanted stimulator, no further research was found on this type of feedback. This may imply that it did not perform well enough to become commercially available, or no further research was performed. Further research on invasive methods to provide feedback may give a better insight in the possibilities of nerve sensing and stimulation for feedback and control. Invasive techniques may however not become as widely accepted, due to their invasive nature, although sensors or stimulators may already be implanted during the amputation.

Incorporating inertial sensors not only in the knee and lower leg, but also in the socket or upper leg may be a useful addition to current MPC knees. This can easily be implemented and was up to $100 \mathrm{~ms}$ earlier in the detection of movement (chapter 6). Further research could also focus on incorporating TFL activity in the control of a prosthesis of a number of subjects. This could give valuable information on the usability of EMG inside a prosthesis. The use of intention detection by EMG could also be further investigated for other activities. The approach by Hoover et al. [42], using muscle co-contraction of the vastus lateralis and the biceps femoris to control the impedance of the prosthetic knee, could also be further explored. It was however still rather slow and a lot of training was needed [42]. Although this amount of training may be accepted by some amputees, as a large number of amputees is above the age of 60 [2], this may not become a widely used approach. For the benefit of the patient it is therefore recommended to keep the control of the prosthesis as simple as possible and very reliable. If an amputee with mechanical prosthetic knee falls due to a knee buckle, because he loaded an unlocked knee, he is likely to blame himself. If the same amputee trusts a high tech, EMG controlled prosthesis and falls, he is likely (and rightfully so?) to blame the prosthesis. 
Chapter 8 


\section{Bibliography}

[1] L. Cremers and I. van der Molen, "Behandel protocol amputatiepatienten voor fysiotherapeuten," Tech. rep., Hogeschool Amsterdam (2005).

[2] J. Geertzen and J. Rietman, Amputatie en prothesiologie van de onderste extremiteit (LEMMA, Den Haag, 2008), 2nd ed.

[3] E. Prinsen, M. Nederhand, and J. Rietman, "Adaptation strategies of the lower extremities of patients with transtibial or transfemoral amputation level during level walking: a systematic review," Achives of Physical Medicine and Rehabilitation 92, 1311-1325 (2011).

[4] Ottobock, "http://www.ottobock.de/," (2013).

[5] Ossur, "http://www.ossur.nl/," (2013).

[6] A. Vrieling, "Movement and balance control in lower limb amputees," Ph.D. thesis (2009).

[7] W. Miller, M. Speechley, and A. Deathe, "The prevalence and risk factors of falling and fear of falling among lower extremity amputees," Archives of Physical Medicine and Rehabilitation 82, 1031-1037 (2001).

[8] W. Miller and A. Deathe, "A prospective study examining balance confidence among individuals with lower limb amputation," Disability \& Rehabilitation 26, 875-881 (2004).

[9] R. Waters, J. Perry, D. Antonelli, and H. Hislop, "Energy cost of walking of amputees: the influence of level of amputation," Journal of Bone and Joint Surgery - Series A 58, 42-46 (1976).

[10] E. Kandel, J. Schwartz, and T. Jessel, Principles of neural science (McGraw-Hill, London, 2000), 4th ed.

[11] M. Paasuke, J. Ereline, and H. Gapeyeva, "Neuromuscular fatigue during repeated exhaustive submaximal static contractions of knee extensor muscles in endurance-trained, power-trained and untrained men," Acta Physiologica Scandinavica 166, 319-326 (1999). 
[12] S. Zhou, "Acute effect of repeated maximal isometric contraction on electromechanical delay of knee extensor muscle," Journal of Electromyography and Kinesiology 6, 117-127 (1996).

[13] D. Winter, Biomechanics and motor control of human movement (John Wiley \& Sons Inc., Hoboken, New Jersey, 2004), 3rd ed.

[14] R. Unal, S. Behrens, R. Carloni, E. Hekman, S. Stramigioli, and H. Koopman, "Prototype design and realization of an innovative energy efficient transfemoral prosthesis," in "IEEE RAS and EMBS International Conference on Biomedical Robotics and Biomechatronics (BioRob)," (2010), pp. 191-196.

[15] B. Hafner, L. Willingham, N. Buell, K. Allyn, and D. Smith, "Evaluation of function, performance, and preference as transfemoral amputees transition from mechanical to microprocessor control of the prosthetic knee," Archives of Physical Medicine and Rehabilitation 88, 207-217 (2007).

[16] V. Kalanovic, D. Popovic, and N. Skaug, "Feedback error learning neural network for trans-femoral prostheses," IEEE Transactions on Rehabilitation Engineering 8, 71-80 (2000).

[17] R. Seymour, B. Engbretson, K. Kott, N. Ordway, G. Brooks, J. Crannel, E. Hichkernell, and K. Wheeler, "Comparison between the cleg microprocessor-controlled prosthetic knee and non-microprocessor control prosthetic knees: A preliminary study of energy expenditure, obstacle course performance, and quality of life survey," Prosthetics and Orthotics International 31, 51-61 (2007).

[18] R. Williams, A. Turner, M. Orendurff, A. Segal, G. Klute, J. Pecoraro, and J. Czerniecki, "Does having a computerized prosthetic knee influence cognitive performance during amputee walking?" Archives of Physical Medicine and Rehabilitation 87, 989-994 (2006).

[19] D. Winter, "Kinematic and kinetic patterns in human gait: variability and compensating effects," Human Movement Science 3, 51-76 (1984).

[20] D. Winter, The biomechanics and motor control of human gait (University of Waterloo Press, Waterloo, 1987), 1st ed.

[21] J. Rose and J. Gamble, Human walking (Lippincott Williams and Wilkins, Philadelphia, 2006), 3rd ed.

[22] J. Perry, Gait Analyis; normal and pathalogical function (SLACK Incorporated, Thorofare, 1992).

[23] A. Vrieling, H. van Keeken, T. Schoppen, E. Otten, J. Halbertsma, A. Hof, and K. Postema, "Uphill and downhill walking in unilateral lower limb amputees," Gait \& Posture 28, 235-242 (2008).

[24] A. Vrieling, H. van Keeken, T. Schoppen, A. Hof, B. Otten, J. Halbertsma, and K. Postema, "Gait adjustments in obstacle crossing, gait initiation and gait termination after a recent lower limb amputation," Clinical Rehabilitations 23, 659-671 (2009). 
[25] H. van Keeken, A. Vrieling, A. Hof, J. Halbertsma, T. Schoppen, K. Postema, and B. Otten, "Controlling propulsive forces in gait initiation in transfemoral amputees," Journal of Biomechanical Engineering 130, 011002:1-9 (2008).

[26] S. Jaegers, J. Arendzen, and H. de Jongh, "Prosthetic gait of unilateral transfemoral amputees: a kinematic study," Achives of Physical Medicine and Rehabilitation 76, 736-743 (1995).

[27] S. Jaegers, J. Arendzen, and H. de Jongh, "An electromyographic study of the hip muscles of transfemoral amputees in walking," Clinical Orthopaedics and Related Research 328, 119-128 (1996).

[28] D. H. Plettenburg and J. Herder, "Voluntary closing: A promising opening in hand prosthetics," Technology and Disability 15, 85-94 (2003).

[29] S. Carey, R. Dubey, G. Bauer, and M. Highsmith, "Kinematic comparison of myoelectric and body powered prostheses while performing common activities," Prosthetics and Orthotics International 33, 179-186 (2009).

[30] T. B. Inc., "http://www.touchbionics.com," .

[31] T. A. Kuiken, P. Marasco, B. Lock, R. Harden, and J. Dewald, "Redirection of cutaneous sensation from the hand to the chest skin of human amputees with targeted reinnervation," Proceedings of the National Academy of Sciences of the United States of America 104, 20061-20066 (2007).

[32] D. Myers and G. Moskowitz, "Myoelectric pattern recognition for use in the volitional control of above-knee prostheses," IEEE Transactions on Systems, Man and Cybernetics 11, 296-302 (1981).

[33] D. Jin, J. Yang, R. Zhang, R. Wang, and J. Zhang, "Terrain identification for prosthetic knees based on electromyographic signal features," Tsinghua Science \& Technology 11, 74-79 (2006).

[34] H. Hillstrom and G. Moskowitz, "Robust intent recognition for prosthesis control," in "Proceedings of the Annual International Conference of the IEEE Engineering in Medicine and Biology Society," , vol. 14 (1992), vol. 14, pp. 1448-1449.

[35] L. Peeraer, B. Aeyels, and G. Van der Perre, "Development of emg-based mode and intent recognition algorithms for a computer-controlled aboveknee prosthesis," Journal of Biomedical Engineering 12, 178-182 (1990).

[36] F. Zhang, Z. Dou, M. Nunnery, and H. Huang, "Real-time implementation of an intent recognition system for artificial legs," in "33rd ann. int. conf. IEEE EMBS," .

[37] H. Huang, F. Zhang, L. Hargrove, Z. Dou, D. Rogers, and K. Englehart, "Continuous locomotion-mode identification for prosthetic legs based on neuromuscular and mechanical fusion," IEEE Transactions on Biomedical Engineering 58, 2867-2875 (2011). 
[38] J. Hong and M. M.S., "Relationship between socket pressure and emg of two muscles in trans-femoral stumps during gait," Prosthetics and Orthotics International 29, 59-72 (2005).

[39] B. Aeyels, W. Van Petegem, J. Sloten, G. Van Der Perre, and L. Peeraer, "An emg-based finite state approach for a microcomputer-controlled above-knee prosthesis," in "IEEE 17th Annual Conference Engineering in Medicine and Biology Society, 1995," , vol. 2 (1995), vol. 2, pp. 13151316.

[40] L. Hargrove, "Real-time myoelectric control of knee and ankle motions for tranfemoral amputees," Journal of the American Medical Association 305, 1542-1544 (2011).

[41] C. Hoover, G. Fulk, and K. Fite, "Stair ascent with a powered transfemoral prosthesis under direct myoelectric control," IEEE/ASME Transactions on Mechatronics PP, 1-10 (2012).

[42] C. Hoover, G. Fulk, and K. Fite, "Stair ascent with a powered transfemoral prosthesis under direct myoelectric control," IEEE/ASME Transactions on Mechatronics 18, 1191-1200 (2013).

[43] G. Dhillon and K. Horch, "Direct neural sensory feedback and control of a prosthetic arm," IEEE Transactions on Neural Systems and Rehabilitation Engineering 13, 468-472 (2005).

[44] R. Weir, C. Heckathorne, and D. Childress, "Cineplasty as a control input for externally powered prosthetic components," Journal of Rehabilitation Research and Development 38, 357-363 (2001).

[45] J. Yao, C. Carmona, A. Chen, T. Kuiken, and J. Dewald, "Sensory cortical re-mapping following upper-limb amputation and subsequent targeted reinnervation: A case report," in "Proceedings of the Annual International Conference of the IEEE Engineering in Medicine and Biology Society, EMBS," (2011), pp. 1065-1068.

[46] T. Sinkjaer, M. Haugland, and J. Haase, "Natural neural sensing and artificial muscle control in man," Experimental Brain Research 98, 542545 (1994).

[47] K. Yoshida, I. Lewinsky, M. Nielsen, and M. Hylleberg, "Implantation mechanics of tungsten microneedles into peripheral nerve trunks," Medical and Biological Engineering and Computing 45, 413-420 (2007).

[48] F. Clippinger, J. McElhaney, G. Maxwell, D. Vaughn, G. Horton, and L. Bright, "Prosthetic sensory feedback lower extremity," Prosthetics ans Orthotics Clinic 5, 1-3 (1981).

[49] F. Clippinger, A. Seaber, J. McElhaney, J. Harrelson, and G. Maxwell, "Afferent sensory feedback for lower extremity prostheses," Clinical Orthopaedics and Related Research No. 169, 202-208 (1982). 
[50] R. Fan, M. Culjat, K. Chih-Hung, M. Franco, R. Boryk, J. Bisley, E. Dutson, and W. Grundfest, "A haptic feedback system for lower-limb prostheses," IEEE Transactions on Neural Systems and Rehabilitation Engineering 16, 270-277 (2008).

[51] T. Koritnik, A. Koenig, T. Bajd, R. Riener, and M. Munih, "Comparison of visual and haptic feedback during training of lower extremities," Gait \& Posture 32, 540-546 (2010).

[52] B. Darter and J. Wilken, "Gait training with virtual reality-based realtime feedback: Improving gait performance following transfemoral amputation," Physical Therapy 91, 1385-1394 (2011).

[53] L. Yang, P. Dyer, R. Carson, J. Webster, B. Foreman, and S. Bamberg, "Utilization of a lower extremity ambulatory feedback system to reduce gait asymmetry in transtibial amputation gait," Gait \& Posture 36, 631634 (2012).

[54] E. Isakov, "Gait rehabilitation: A new biofeedback device for monitoring and enhancing weight-bearing over the affected lower limb," Europa Medicophysica 43, 21-26 (2007).

[55] R. Mann and S. Reimers, "Kinesthetic sensing for the emg controlled "boston arm"," IEEE Transactions on Man Machine Systems 11, 110115 (1970).

[56] C. Pylatiuk, S. Mounier, A. Kargov, S. Schulz, and G. Bretthauer, "Progress in the development of a multifunctional hand prosthesis," in "International Conference of the IEEE Engineering in Medicine and Biology Society," , vol. 6 (2004), vol. 6, pp. 4260-4263.

[57] H. Witteveen, A. Droog, J. Rietman, and P. Veltink, "Vibro- and electrotactile user feedback on hand opening for myoelectric forearm prostheses," IEEE Transactions on Biomedical Engineering 59, 2219-2226 (2012).

[58] H. Witteveen, J. Rietman, and P. Veltink, "Grasping force and slip feedback through vibrotactile stimulation to be used in myoelectric forearm prostheses," in "Proceedings of the Annual International Conference of the IEEE Engineering in Medicine and Biology Society, EMBS," (2012), pp. 2969-2972.

[59] W. Vos, D. Buma, and P. Veltink, "Optimisation of spatial electrocutaneous display parameters for sensory substitution," in "Proceedings of Eurohaptics," (2004).

[60] K. Kaufman, J. Levine, R. Brey, B. Iverson, S. McCrady, D. Padgett, and M. Joyner, "Gait and balance of transfemoral amputees using passive mechanical and microprocessor-controlled prosthetic knees," Gait \& Posture 26, 489-493 (2007). 
[61] L. Visser, R. Carloni, and S. Stramigioli, "Energy-efficient variable stiffness actuators," IEEE Transactions on robotics Article in press (2011).

[62] B. Vanderborght, N. Tsagarakis, R. Van Ham, I. Thorson, and D. Caldwell, "Maceppa 2.0: compliant actuator used for energy efficient hopping robot chobino 1d," Auton Robot 31, 55-65 (2011).

[63] R. Filippini, S. Sen, and A. Bicchi, "Towards soft robots you can depend on; adaptable compliance," IEEE Robotics \& Automation Magazine 10, 31-41 (2008).

[64] A. Segal, M. Orendurff, G. Klute, M. McDowell, J. Pecoraro, J. Shofer, and J. Czerniecki, "Kinematic and kinetic comparisons of transfemoral amputee gait using c-leg and mauch sns prosthetic knees." Journal of Rehabilitation Research and Development 43, 857-870 (2006).

[65] A. Kuo and J. Donelan, "Dynamic principles of gait and their clinical implications," Journal of the American Physical Therapy Association 90, 157-174 (2010).

[66] S. Lindstedt, T. Reich, P. Keim, and P. LaStayo, "Do muscles function as adaptable locomotor springs?" Journal of Experimental Biology 205, 2211-2216 (2002).

[67] S. Blumentritt, T. Schmalz, and R. Jarasch, "The safety of c-leg: biomechanical tests," Journal of Prosthetics and Orthotics 21, 2-15 (2009).

[68] S. Au, J. Weber, and H. Herr, "Biomechanical design of a powered anklefoot prosthesis," in "IEEE 10th International Conference on Rehabilitation Robotics ICORR," (2007), pp. 298-303.

[69] S. Au and H. Herr, "Powered ankle-foot prosthesis; adaptable compliance," IEEE Robotics \& Automation Magazine 10, 52-59 (2008).

[70] J. Zhu, Q. Wang, and L. Wang, "Pantoe 1: Biomechanical design of powered ankle-foot prosthesis with compliant joints and segmented foot," in "IEEE Int Conf Advanced Intelligent Mechatronics," (2010), pp. 3136 .

[71] T. Sugar, K. Hollander, and J. Hitt, Walking with springs, vol. 7976 (SPIE, 2011).

[72] B. Vanderborght, R. Van Ham, D. Lefeber, T. Sugar, and K. Hollander, "Comparison of mechanical design and energy consumption of adaptable, passive-compliant actuators," The International Journal of Robotics Research 28, 90-103 (2009).

[73] X. Shen and M. Goldfarb, "Simultaneous force and stiffness control af a pneumatic actuator," Journal of Dynamic Systems, Measurement and Control 129, 425-434 (2007).

[74] D. Braun and M. Goldfarb, "A control approach for actuated dynamic walking in biped robots," IEEE Transactions on Robotics 25, 1292-1303 (2009). 
[75] S. Stramigioli, G. van Oort, and E. Dertien, "A concept of a new energy efficient actuator," in "Proc. IEEE/ASME Int Conf on Adv Intel Mech," (2008), pp. 671-675.

[76] B. Lawson, H. Varol, F. Sup, and M. Goldfarb, "Stumble detection and classification for an intelligent transfemoral prosthesis," (2010), pp. 511514 .

[77] J. Duysens, V. Beerepoot, P. Veltink, V. Weerdesteyn, and B. SmitsEngelsman, "Proprioceptive perturbations of stability during gait," Neurophysiologie Clinique/Clinical Neurophysiology 38, 399-410 (2008).

[78] G. Staude, C. Flachenecker, M. Daumer, and W. Wolf, "Onset detection in surface electromyographic signals: a systematic comparison of methods," EURASIP J. Appl. Signal Process. 2001, 67-81 (2001).

[79] X. Li, P. Zhou, and A. Aruin, "Teagerkaiser energy operation of surface emg improves muscle activity onset detection," Annals of Biomedical Engineering 35, 1532-1538 (2007).

[80] R. Merletti and P. Parker, Electromyography (Wiley Interscience, Hoboken, 2004).

[81] B. S. Farber and J. Jacobson, "An above-knee prosthesis with a system of energy recovery: A technical note," Journal of Rehabilitation Research and Development 32, 337-348 (1995).

[82] B. Aeyels, L. Peeraer, J. Vander Sloten, and G. Van der Perre, "Development of an above-knee prosthesis equipped with a microcomputercontrolled knee joint: first test results," Journal of Biomedical Engineering 14, 199-202 (1992).

[83] A. Kuo, "The six determinants of gait and the inverted pendulum analogy: A dynamic walking perspective," Human Movement Science 26, 617-656 (2007).

[84] B. Koopman, H. Grootenboer, and H. de Jongh, "An inverse dynamics model for the analysis, reconstruction and prediction of bipedal walking," Journal of Biomechanics 28, 1369-1376 (1995).

[85] O. Huat, D. Ghista, N. Beng, and T. John, "Optimal stride frequency computed from the double-compound pendulum of the leg, and verified experimentally as the preferred stride frequency of jogging," Int. J. Comput. Appl. Technol. 21, 46-51 (2004).

[86] J. Bowker, Atlas of limb prosthetics: surgical, prosthetic, and rehabilitation principles (American Academy of Orthopaedic Surgeons, Rosemont, 2002), 2nd ed.

[87] F. Farahmand, T. Rezaeian, R. Narmani, and P. H. Dinan, "Kinematic and analysis of the gait cycle of above-knee amputees," Scientia Iranica 13, 261-271 (2006). 
[88] J. Johansson, D. Sherrill, P. Riley, P. Bonato, and H. Herr, "A clinical comparison of variable-damping and mechanically passive prosthetic knee devices," American Journal of Physical Medicine \& Rehabilitation 84, 563-575 (2005).

[89] M. Linden, van der, S. Solomonidis, W. Spence, N. Li, and J. Paul, "A methodology for the studying the effects of various types of prosthetic feet on the biomechanics of trans-femoral amputee gait," Jounal of Biomechanics 32, 877-889 (1999).

[90] M. Bellmann, T. Schmalz, and S. Blumentritt, "Comparative biomechanical analysis of current microprocessor-controlled prosthetic knee joints," Archives of Physical Medicine and Rehabilitation 91, 644-652 (2010).

[91] L. Frossard, R. Tranberg, E. Haggstrom, M. Pearcy, and R. Branemark, "Load on osseointegrated fixation of a transfemoral amputee during a fall: loading, descent, impact and recovery analysis." Prosthetics and Orthotics International 34, 85-96 (2010).

[92] C. Ertekin, F. Bademkiran, S. Yalin, N. Karapinar, and H. Uysal, "Withdrawal reflexes in adductor muscles elicited by electrical and magnetic stimulation of the obturator nerve," Muscle \& Nerve 38, 1490-1497 (2008).

[93] M. Bobbert and G. van Ingen Schenau, "Coordination in vertical jumping," Journal of Biomechanics 21, 249-262 (1988).

[94] J. Perry, J. Burnfield, C. Newsam, and P. Conley, "Energy expenditure and gait characteristics of a bilateral amputee walking with c-leg prostheses compared with stubby and conventional articulating prostheses." Achives of Physical Medicine and Rehabilitation 85, 1711-17 (2004).

[95] L. Nolan and A. Lees, "The functional demands on the intact limb during walking for active trans-femoral and trans-tibial amputees," Prosthetics and Orthotics International 24, 117-125 (2000).

[96] R. Seroussi, A. Gitter, J. Czerniecki, and K. Waever, "Mechanical work adaptations of above-knee amputee ambulation," Achives of Physical Medicine and Rehabilitation 77, 1209-1214 (1996).

[97] H. Huang, T. Kuiken, and R. Lipschutz, "A stratefy for identification locomotion modes using surface elecotmyography," IEEE Transactions on Biomedical Engineering 56, 65-73 (2009).

[98] T. Bae, H. Choi, and M. Mun, "Level walking and stair climbing gait in above-knee amputees," Journal of Medical Engineering \& Technology 22, 130-135 (2009).

[99] S. Huang and D. Ferris, "Muscle activation patterns during walking from transtibial amputees recorded within the residual limb-prosthetic interface," Journal of NeuroEngineering and Rehabilitation 9, 1-16 (2012). 
[100] H. Hermens, B. Freriks, R. Merletti, D. Stegeman, J. Blok, G. Rau, C. Disselhorst-Klug, and G. Hagg, European recommendations for surface electromyography (Roessingh Research and Development, Enschede, the Netherlands, 1999).

[101] H. Luinge, P. H. Veltink, and C. Baten, "Ambulatory measurement of arm orientation," Journal of Biomechanics 40, 78-85 (2007).

[102] E. Wentink, S. Beijen, H. Hermens, J. Rietman, and P. Veltink, "Intention detection of gait initiation using emg and kinematic data," Gait \& Posture pp. 223-8 (2012).

[103] T. Watanabe, H. Saito, E. Koike, and K. Nitta, "A preliminary test of measurement of joint angles and stride length with wireless inertial sensors for wearable gait evaluation system," Computational Intelligence and Neuroscience (2011).

[104] A. Chan and G. Green, "Myoelectric control development toolbox," in "30th Conference of the Canadian Medical \& Biological Engineering Society," (Toronto, Canada, 2007).

[105] M. Zardoshti-Kermani, B. Wheeler, K. Badie, and R. Hashemi, "Emg feature evaluation for movement control of upper extremity prostheses," IEEE Transactions on Rehabilitation Engineering 3, 324-333 (1995).

[106] J. Mickelborough, M. van der Linden, R. Tallis, and A. Ennos, "Muscle activity during gait initiation in normal elderly people," Gait \& Posture 19, 50-57 (2004).

[107] C. Hershler and M. Morris, "An optimality criterion for processing electromyography (emg) signals relating to human locomotion," IEEE Transactions on Biomedical Engineering 25, 413-420 (1978).

[108] A. Malone, D. Meldrum, J. Gleeson, and C. Bolger, "Reliability of surface electromyography timing parameters in gait in cervical spondylotic myelopathy," Journal of Electromyography and Kinesiology 21, 1004-1010 (2011).

[109] A. Nene, C. Byrne, and H. Hermens, "Is rectus femoris really a part of quadriceps? assessment of rectus femoris function during gait in ablebodied adults," Gait \& Posture 20 (2004).

[110] C. Byrne, G. Lyons, A. Donnelly, D. O'Keeffe, H. Hermens, and A. Nene, "Rectus femoris surface myoelectric signal cross-talk during static contractions," Journal of Electromyography and Kinesiology 15, 564-575 (2005).

[111] K. Granata, D. Padua, and M. Abel, "Repeatability of surface emg during gait in children," Gait \& Posture 22, 346-350 (2005).

[112] A. Burden, M. Trew, and V. Balzopoulos, "Normalisation of gait emgs: a re-examination," Journal of Electromyography and Kinesiology 13, 519$532(2003)$. 
[113] V. Monaco, A. Ghionzoli, and S. Micera, "Age-related modifications of muscle synergies and spinal cord activity during locomotion," Journal of Neurophysiology 104, 2092-2102 (2010).

[114] J. Buurke, H. Hermens, C. Erren-Wolters, and A. Nene, "The effect of walking aids on muscle activation patterns during walking in stroke patients," Gait \& Posture 22, 164-170 (2005).

[115] L. Frossard, J. Beck, M. Dillon, and J. Evans, "Development and preliminary testing of a device for the direct measurement of forces and moments in the prosthetic limb of transfemoral amputees during activities of daily living," JPO: Journal of Prosthetics and Orthotics 15, 135-142 (2003).

[116] A. Vrieling, H. van Keeken, T. Schoppen, E. Otten, J. Halbertsma, A. Hof, and K. Postema, "Uphill and downhill walking in unilateral lower limb amputees," Gait \& Posture 28, 235-242 (2008).

[117] E. Wolf, V. Everding, A. Linberg, B. Schnall, J. Czerniecki, and J. Gambel, "Assessment of transfemoral amputees using c-leg and power knee for ascending and descending inclines and steps," Journal of Rehabilitation Research and Development 49, 831-842 (2012).

[118] H. Hobara, Y. Kobayashi, T. Nakamura, N. Yamasaki, K. Nakazawa, M. Akai, and T. Ogata, "Lower extremity joint kinematics of stair ascent in transfemoral amputees," Prosthetics and Orthotics International 35, 467-472 (2011).

[119] T. Schmalz, S. Blumentritt, and B. Marx, "Biomechanical analysis of stair ambulation in lower limb amputees," Gait \& Posture 25, 267-278 (2007).

[120] K. Kaufman, J. Levine, R. Brey, B. Iverson, S. McCrady, D. Padgett, and M. Joyner, "Gait and balance of transfemoral amputees using passive mechanical and microprocessor-controlled prosthetic knees," Gait \& Posture 26, 489-493 (2007).

[121] D. Smith, "The transfemoral amputation level, part 1," inMotion 14, 54-58 (2004).

[122] A. McIntosh, K. Beatty, L. Dwann, and D. Vickers, "Gait dynamics on an inclined walkway," Journal of Biomechanics 39, 2491-2502 (2006).

[123] S. D. Prentice, E. N. Hasler, J. J. Groves, and J. S. Frank, "Locomotor adaptations for changes in the slope of the walking surface," Gait \& Posture 20, 255-265 (2004).

[124] A. Leroux, J. Fung, and H. Barbeau, "Adaptation of the walking pattern to uphill walking in normal and spinal-cord injured subjects," Experimental Brain Research 126, 359-368 (1999).

[125] A. N. Lay, C. J. Hass, T. Richard Nichols, and R. J. Gregor, "The effects of sloped surfaces on locomotion: An electromyographic analysis," Journal of Biomechanics 40, 1276-1285 (2007). 
[126] M. Kuster, S. Sakurai, and G. Wood, "Kinematic and kinetic comparison of donhill and level walking," Clinical Biomechanics 10, 79-84 (1995).

[127] A. N. Lay, C. J. Hass, and R. J. Gregor, "The effects of sloped surfaces on locomotion: A kinematic and kinetic analysis," Journal of Biomechanics 39, 1621-1628 (2006).

[128] S. Reid, S. Lynn, R. Musselman, and P. Costigan, "Knee biomechanics of alternate stair ambulation patterns," Medicine \& Science in Sports \& Exercise 39, 2005-11 (2007).

[129] S. Jones, P. Twigg, A. Scally, and J. Buckley, "The mechanics of landing when stepping down in unilateral lower-limb amputees," Clinical Biomechanics 21, 184-193 (2006).

[130] S. Jaegers, "The morphology and functions of the muscles around the hip joint after a unilateral transfemoral amputation," Ph.D. thesis (1993).

[131] K. Kaufman, S. Frittoli, and C. Frigo, "Gait asymmetry of transfemoral amputees using mechanical and microprocessor-controlled prosthetic knees," Clinical Biomechanics 27, 460-465 (2012).

[132] A. Tura, L. Rocchi, M. Raggi, A. Cutti, and L. Chiari, "Recommended number of strides for automatic assessment of gait symmetry and regularity in above-knee amputees by means of accelerometry and autocorrelation analysis," Journal of NeuroEngineering and Rehabilitation 9, 11 (2012).

[133] R. Gailey, K. Allen, J. Castles, J. Kucharik, and M. Roeder, "Review of secondary physical conditions associated with lower-limb amputation and long-term prosthesis use," Journal of Rehabilitation Research and Development 45, 15-30 (2008).

[134] R. Mann, J. Hagy, V. White, and D. Liddell, "The initiation of gait," J Bone and Joint Surgery 61, 232-239 (1979).

[135] V. Michel and R. Chong, "The strategies to regulate and to modulate the propulsive forces during gait initiation in lower limb amputees," Experimental Brain Research 158, 356-365 (2004).

[136] A. Erdemir and S. Piazza, "Rotational foot placement specifies the lever arm of the ground reaction force during the push-off phase of walking initiaiton," Gait and Posture 15, 212-219 (2002).

[137] A. Queralt, J. Valls-Sole, and J. Castellote, "Speeding up gait initiation and gait-pattern with a startling stimulus," Gait \& Posture 31, 185-190 (2010).

[138] K. Ha, H. Varol, and M. Goldfarb, "Volitional control of a prosthetic knee using surface electromyography," IEEE Trans Biomed Eng 58, 144-151 (2011). 
[139] B. Najafi, D. Miller, B. Jarett, and J. Wrobel, "Does footwear type impact the number of steps required to reach gait steady state?:an innovative look at the impact of foot orthoses on gait initiation," Gait \& Posture 32, 29-33 (2010).

[140] C. Tokuno and J. Eng, "Gait initiation is dependent on the function of the paretic trailing limb in individuals with stroke," Gait \& Posture 24, 424-428 (2006).

[141] A. Hansen, S. Miff, D. Childress, S. Gard, and M. Meier, "Net external energy of the biologic and prosthetic ankle during gait initiation," Gait \& Posture 31, 13-17 (2010).

[142] D. Zwartjes, C. Heida, J. van Vugt, J. Geelen, and P. H. Veltink, "Ambulatory monitoring of activities and motor symptomes in parkinson's disease," IEEE Trans Biomed Eng 57, 2778-2786 (2010).

[143] G. Staude and W. Wolf, "Objective motor response onset detection in surface myoelectric signals," Med Eng \& Phys 21, 449-467 (1999).

[144] D. Roetenberg, J. Buurke, P. Veltink, A. Forner Cordero, and H. Hermens, "Surface electromyography analysis for variable gait," Gait \& Posture 18, 109-117 (2003).

[145] A. Willemsen, F. Bloemhof, and H. Boom, "Automatic stance-swing phase detection from accelerometer data for peroneal nerve stimulation," IEEE Transactions on Biomedical Engineering 37, 1201-1208 (1990).

[146] B. Peerdeman, D. Boere, H. Witteveen, R. in 't Veld, H. Hermens, S. Stramigioli, H. Rietman, P. Veltink, and S. Misra, "Myoelectric forearm prostheses: state of the art from a user-centered perspective," Journal of Rehabilitation Research and Development 48, 719-737 (2011).

[147] W. Vos, D. Buma, and P. Veltink, "Towards the optimisation of spatial electrocutaneous display parameters for sensory substitution," Tech. rep., Research School 'Integrated Biomedical Engineering for Restoration of Human Function' (iBME) / University of Twente (2002).

[148] D. Buma and P. Veltink, "Effect of stimulation level on sensation during continuous electrocutaneous stimulation," (2001).

[149] J. Stepien, S. Cavenett, L. Taylor, and M. Crotty, "Activity levels among lower-limb amputees: self-report versus step activity monitor," Archives of Physical Medicine and Rehabilitation 88, 896-900 (2007).

[150] K. Kaczmarek, J. Webster, P. Bach-y Rita, and W. Tompkins, "Electrotactile and vibrotactile displays for sensory substitution systems," IEEE Transactions on Biomedical Engineering 38, 1-16 (1991).

[151] K. Kaczmarek, "Electrotactile adaptation on the abdomen: preliminary results," IEEE Transactions on Rehabilitation Engineering 8, 499-505 (2000). 
[152] L. Korner, "Afferent electrical nerve stimulation for sensory feedback in hand prostheses," Ph.D. thesis, University of Goteborg (1979).

[153] D. Buma, J. Buitenweg, and P. Veltink, "Intermittent stimulation delays adaptation to electrocutaneous sensory feedback," IEEE Transactions on Neural Systems and Rehabilitation Engineering 15, 435-441 (2007).

[154] A. Higashiyama and T. Tashiro, "Magnitude estimates for electrical pulses: evidence for two neural mechanisms." Perception \& Psychophysics 45, 537-549 (1989).

[155] S. Weinstein, Intensive and extensive aspects of tactile sensitivity as a function of body part, sex, and laterality, The Skin Senses (Thomas books, Springfield IL, 1968).

[156] A. Schillings, B. van Wezel, T. Mulder, and J. Duysens, "Muscular responses and movement strategies during stumbling over obstacles," Journal of Neurophysiology 83, 2093-2102 (2000).

[157] A. Forner Cordero, H. Koopman, and F. van der Helm, "Multiple-step strategies to recover from stumbling perturbations," Gait \& Posture 18, 47-59 (2003).

[158] E. Wentink, A. Mulder, J. Rietman, and P. Veltink, "Vibrotactile stimulation of the upper leg: Effects of location, stimulation method and habituation," in "33rd Annual International Conference of the IEEE Engineering in Medicine and Biology Society, EMBS 2011," pp. 1668-1671.

[159] D. Mahns, N. Perkins, V. Sahai, L. Robinson, and M. Rowe, "Vibrotactile frequency discrimination in human hairy skin," Journal Neurophysiology 95, 1442-1450 (2006).

[160] R. Cholewiak, "The perception of tactile distance: Influences of body site, space and time," Perception 28, 851-875 (1999).

[161] R. Verrillo, "Psychophysics of vibrotactile stimulation," The Journal of the Acoustical Society of America 77, 225-232 (1985).

[162] L. Alfring, E. Wentink, H. Witteveen, E. Hekman, and P. Veltink, "Application of vibrotactile stimulation in prostheses," Tech. rep., University of Twente, Enschede, the Netherlands (2012).

[163] R. Kosinski, "A literature review on reaction time, http : //biae.clemson.edu/bpc/bp/lab/110/reaction.htm\#kinds," (2005).

[164] R. Koning, E. Wentink, R. Unal, H. Rietman, P. Veltink, and H. Koopman, "Investigation of the optimal electrotactile feedback for transfemoral prostheses," Tech. rep., University of Twente, Enschede, the Netherlands (2011).

[165] P. Steenbergen, J. Buitenweg, E. van der Heide, and P. H. Veltink, "Characterization of a bimodal electrocutaneous stimulation device," in "eMBEC," (2008), p. 5. 
[166] E. Talsma Kerkdijk, E. Wentink, E. Prinsen, E. van Asseldonk, and P. Veltink, "Effect of feedback on perturbed transfemoral prosthetic walking," Tech. rep., University of Twente, Enschede, the Netherlands (2012).

[167] M. Garca-Prez, "A cautionary note on the use of the adaptive up-down method," Journal of the Acoustical Society of America 130, 2098-2107 (2011).

[168] T. Cornsweet, "The staircase-method in psychophysics," The American Journal of Psychology 75, 485-491 (1962).

[169] J. Eng, D. Winter, and A. Patla, "Strategies for recovery from a trip in early and late swing during human walking," Experimental Brain Research 102, 339-349 (1994). 


\section{Summary}

The Reflex-leg project aims at designing a prosthesis that could be controlled reflexively and energy efficiently without invasive procedures. Introducing the user into the control and feedback loop may make the prosthesis more user-friendly and could increase confidence in the prosthesis. In this thesis, research concerning reflexive control of upper leg prostheses is presented. In three parts different studies on reflexive control combined with energy efficient variable stiffness actuation (VSA), voluntary prosthetic control and feedback in upper leg prostheses are presented.

Part I describes the results of a model study on a new concept of an energy efficient VSA controlled knee combined with reflexive control. The concept uses the principle that the output stiffness of a spring can be changed without changing the energy stored in the elastic elements of the spring. The usability of this concept was evaluated by modeling disturbance rejections by the VSA controlled knee. The simulations predicted that energy efficient VSA can be useful for the control of prosthetic knees, but that reflexive control is too slow.

For Part II spatio-temporal, kinematic and EMG measurements were performed on non-amputees and (at the residual limb of) amputees during level, slope and step-by-step stair walking. First, the adaptations found in amputees during these three activities were investigated. Changes were found in the duration of the stance and swing phases as well as the duration of muscle activity. Muscle activity patterns in amputees were largely the same as in controls, but differed mainly in the stance to swing transition. All changes are likely to compensate for reduced foot progression, the lack of actuation of the prosthetic knee and ankle, and to increase socket fitting. The intact leg is used more 
extensively for stability and support. Secondly, the possibilities of using kinematic data and EMG for intention detection in controls and amputees were investigated. Intention detection could improve the control of the prosthetic knee during gait initiation. In non-amputees, initial swing could be predicted up to 130-260 ms in advance when using either kinematic data or EMG of several muscles. In amputees kinematic data was able to detect initial movement of both leading limbs. EMG of the tensor fascia latae was able to predict the initial movement of the prosthetic leading limb up to $138 \mathrm{~ms}$ in advance. For the intact limb leading, EMG was not found to be of additional value. However, placing an inertial sensor at the upper leg may improve the detection speed, for both leading limb conditions.

Part III describes four studies addressing feedback in upper leg prosthesis, all performed in non-amputees. Vibrotactile feedback applied to the upper leg at different locations showed that the posterior and medial side of the upper leg are more sensitive to vibrations. Information provided to subjects by electrotactile and vibrotactile stimulation in an array at the upper leg appeared to be difficult to interpret. Vibrotactile feedback was also applied to the upper leg with and without a socket. When providing space for the vibrator to vibrate the socket did not alter the perception, but it did increase the reaction time. Error-based feedback was provided during disturbed walking on a simulated prosthesis. Responses to disturbances were up to $40 \mathrm{~ms}$ earlier with auditive feedback than without feedback. Using electrotactile feedback no changes in the responses were found.

From this thesis it can be concluded that it is unlikely that control of a transfemoral prosthesis will be safe and fast enough, if the user is made part of the reflexive control loop by means of non-invasive feedback and EMG control. VSA showed to be a worth while principle that can become useful. Amputees adapt their muscle activity patterns to their prosthesis and to their new walking pattern. The intact limb plays a important role by compensating for the lack of control over the prosthetic knee and ankle, and by increasing stability. Control of a prosthetic knee can benefit from inertial sensors at the upper leg, rather than solely in the lower leg. So far transferring useful information within given time constraints to an amputee through electrotactile and vibrotactile feedback applied at the upper leg was unsuccessful. However, vibrotactile feedback can be used inside a socket under weight bearing conditions. 


\section{Samenvatting}

Het Reflex-leg project had als doel een bovenbeenprothese te ontwerpen die reflexief aangestuurd kon worden en energie efficint zou zijn, zonder (invasieve) ingrepen bij de amputatiepatint. De gebruiker als onderdeel van de aansturings- en feedback lus zou de prothese gebruiksvriendelijker kunnen maken en het vertrouwen in de prothese kunnen vergroten. In dit proefschrift is de reflexieve aansturing van een bovenbeenprothese in drie delen onderzocht. Het eerste deel betreft de reflexieve aansturing in combinatie met een actuator waarvan de stijfheid energie efficint gevarieerd kan worden, een zogenaamde variabele stijfheidsactuator (VSA). De andere twee delen betreffen respectievelijk de prothese-aansturing door de gebruiker en feedback van de prothese naar de gebruiker.

Deel I beschrijft de resultaten van een modelstudie over een nieuw concept: een energie-efficinte en met VSA aangestuurde knie in combinatie met reflexieve aansturing. Het concept is gebaseerd op het principe dat de uitgangsstijfheid van een veer veranderd kan worden zonder dat de energie opgeslagen in de elastische elementen van de veer wordt veranderd. De bruikbaarheid van dit concept is gevalueerd door het opvangen van verstoringen tijdens het lopen door de VSA aangestuurde knie te modeleren. De simulaties voorspelden dat energie efficinte VSA bruikbaar kan zijn voor aansturing van protheseknien, maar dat reflexieve aansturing te langzaam is.

Voor Deel II zijn bewegings- en EMG metingen verricht bij gezonde proefpersonen en (bij het aangedane been van) amputatiepatinten tijdens vlak, trap en helling lopen. Deel II beschrijft ten eerste de adaptaties die in amputatiepatinten zijn gevonden bij de drie activiteiten. Adaptaties zijn gevonden in de duur van de stand en zwaai fases en in de duur van de spieractivatie. Spier activatiepatronen in patinten waren 
grotendeels gelijk aan die in gezonde proefpersonen. Verschillen betroffen vooral de overgang van de stand- naar zwaaifase. Alle verschillen van de bewegingen en de spieractivatiepatronen bij patinten in vergelijking met gezonde proefpersonen zijn waarschijnlijk ter compensatie van de verminderde voortgang over de voet, het gebrek aan aansturing in de protheseknie en -enkel en om de koker beter aan te laten sluiten. Het niet aangedane been wordt meer dan voorheen gebruikt voor steun en stabiliteit. Vervolgens worden in dit deel de mogelijkheden bekeken om bewegings- en EMG signalen voor intentiedetectie te gebruiken bij gezonde proefpersonen en patinten. Dit zou de aansturing van de initiatie van de loopbeweging kunnen verbeteren. In gezonde proefpersonen lieten zowel beweging als EMG van verschillende spieren zien dat intentiedetectie $130-260 \mathrm{~ms}$ voor de start van de zwaaifase mogelijk is. In amputatiepatinten konden de bewegingssignalen de eerste beweging in de benen detecteren, ongeacht met welk been werd gestart. EMG van de tensor facia latae kon de eerste beweging van het startende prothesebeen tot $138 \mathrm{~ms}$ van te voren voorspellen. Wanneer het gezonde been startte was het EMG signaal niet van toegevoegde waarde voor intentie detectie. Een inertile sensor op het prothesebovenbeen zou de detectietijd kunnen verkorten, ongeacht welk been start met lopen.

Deel III beschrijft vier studies over feedback in bovenbeenprotheses, allemaal uitgevoerd bij gezonde proefpersonen. Vibrotactiele feedback op verschillende locaties van het bovenbeen toonde aan dat de posterior en mediale kant van het bovenbeen gevoeliger zijn voor vibraties. Informatievoorziening aan proefpersonen door middel van een rij van elektrotactiele of vibrotactiele stimulaties bleek moeilijk interpreteerbaar. Vibrotatiele feedback is met en zonder koker op het bovenbeen aangeboden. Hieruit bleek dat wanneer de vibrator voldoende ruimte krijgt deze in een koker voelbaar is, maar dat de reactie op een stimulus later komt. Ten slotte is de bruikbaarheid van feedback gegeven bij detectie van een verstoring tijdens het lopen onderzocht. De reacties op auditieve feedback waren tot $40 \mathrm{~ms}$ eerder dan die zonder feedback of met elektrotactiele feedback.

Aan de hand van de studies in dit proefschrift kan geconcludeerd worden dat reflexieve aansturing van de prothese door de gebruiker met de onderzochte niet-invasieve modaliteiten niet snel genoeg zal zijn. De modelstudie voorspelt dat VSA wel een bruikbaar principe is voor protheseknien wanneer op verstoringen moet worden gereageerd. Amputatiepatinten passen hun spieractivatiepatroon aan op hun nieuwe looppatroon. Het niet aangedane been speelt hierin een grote rol: het compenseert voor het gebrek aan controle over de protheseknie en -enkel 
en verzorgt een grotere stabiliteit. Aansturing van de protheseknie zou verbeterd kunnen worden door inertile sensoren op het prothesebovenbeen te plaatsen, in plaats van alleen op het onderbeen. Er is geen geschikte methode gevonden om bruikbare informatie door middel van vibro- en elektrotactiele stimulatie van de knie naar de gebruiker terug te koppelen. Vibrotactiele stimulatie kan echter wel in de koker worden toegepast. 


\section{Dankwoord}

Zo! Het heeft "ff" geduurd, maar het is dan nu eindelijk zover, ik kan aan het dankwoord beginnen. Bijna niet te geloven dat het nu dan echt af is. Nou wordt het natuurlijk tijd om te bedenken wie mij allemaal hebben bijgestaan, gesteund en uit de soms diepe putten hebben gesleept.

Het begin is natuurlijk het gemakkelijkst met promotoren Peter en Hans. Peter je was altijd kritisch en nee dat werd je zeker in het begin niet altijd in dank afgenomen, maar als ik nu sommige dingen teruglees, waardeer ik het meer dan ooit en weet ik dat het erg zinvol was. Jouw meestal snelle feedback, al vond jezelf meestal dat het sneller kon, heeft mij altijd bij de les gehouden. Ik wil je bedanken voor onze goede samenwerking en je eeuwige uithoudingsvermogen om mijn teksten (af en toe echt rommel) van feedback en commentaar te voorzien.

Hans, tja jij bent weer een heel ander verhaal. Jij vond vaak dat je "niet technisch" genoeg was om sommige berekeningen of methodes van commentaar te voorzien, dus die werden dan gemarkeerd met "snap ik toch niet, sla ik over". Echter, jouw kennis op het gebied van de revalidatie en de klinische aspecten, gaf vaak terecht aanleiding tot discussie en stof tot nadenken en bracht vaak ideeen en nieuwe inzichten die Peter en ik samen niet bedacht hadden (als technici). Hiervoor mijn dank!

Halverwege mijn project kwam Erik officieel als "bewegingswetenschapper" bij het project. Dat resulteerde vooral in humor tijdens metingen en besprekingen en een goede (kritische) sparringpartner voor allerlei delen van mijn onderzoek. Ik ben blij dat jij vaak een eerste blik op artikelen e.d. wilde werpen en heb met veel genoegen met je samengewerkt. Verder heb ik veel te danken aan de revalidatieartsen 
Tjerk de Ruijter en Hans Konter voor de tijd en moeite die zij hebben gestoken in het vinden van protheselopers. Ook Jeroen Olsman van het RRT heeft de nodige tijd en moeite gestoken in het maken van protheseonderdelen.

Uiteraard heb ik tijdens mijn onderzoek ook vele "slachtoffers" gemaakt. Tijdens diverse experimenten zijn velen behangen met stickers, kastjes, tape, martelwerktuigen (Sabine!), simulatie protheses en vele andere kabeltjes. Om dan vervolgens nog te moeten traplopen of bewerkt te worden met elektrostimulatie en/of vibratie. Iedereen die ooit heeft meegewerkt aan een van de experimenten, inmiddels te veel om allemaal op te noemen, bedankt! Natuurlijk vallen onder deze "slachtoffers" ook de protheselopers die met veel geduld aan de experimenten hebben meegedaan. Heren Hovenier, Braakman, Lambers, Oude Engberink en Mulder, en mevrouw van der Wal, mijn respect en ik ben jullie zeer erkentelijk voor de medewerking. Aan studenten heb ik ook nooit een gebrek gehad. Sabine was de eerste en Vincent de laatste, daartussen zaten: Renske, Freek, Lisette, Ewoud, Eline en Alex. Jullie hebben allemaal een grote bijdrage geleverd aan mijn promotie en mijn leven weer een stuk makkelijker gemaakt. Van een aantal van jullie is het werk zelfs gepubliceerd of wordt publicatie verwacht. Grote hulde aan jullie allemaal!

Aan technische ondersteuning ook geen gebrek. Voor een kapotte $\mathrm{PC}$, een of ander systeem waarmee je protheselopers kon laten struikelen of gewoon de afleiding tijdens de koffiepauzes, Marcel en Ed jullie stonden altijd klaar om de oplossing te bieden, heren respect! Het kloppend hart van BSS is natuurlijk Wies, zonder jou loopt alles in de soep en jij hebt me dan ook vaak wat soep bespaard hetgeen altijd zeer gewaardeerd werd!

Tevens heb ik in 5 jaar tijd heel wat kamergenoten versleten. Voor korte tijd deelde ik een kamer met: Martin, Remi, Karin, Betty en Josien. Heidi en Victor hebben mij langere tijd moeten verduren en ik wil jullie dan ook bedanken voor het eeuwige vermaak en de steun van jullie kant. Heidi bedankt voor de fantastische samenwerking al die tijd en nu voor het paranimfen!

Dankzij een door mij "verloren" weddenschap met Peter en Daphne heb ik mogen genieten van een heerlijk etentje, ik geniet er nog steeds van! Ook de rest van BSS wil ik graag bedanken voor de gezellige tijd die ik bij jullie gehad heb. Irina, thank you, for your never ending excitement in taking pictures (of everything ;-)). IJs eten en lunchen met de Aios 
van BSS heeft bijgedragen aan sommige memorabele momenten. Jongens, wanneer gaat de vd Poel weer open?

Dan wil ik nog wat mensen bedanken die vooral tijdens de buitenschoolse activiteiten hebben bijgedragen aan mijn algehele welzijn. Met jullie allen heb ik menig (klim)avontuur beleefd en ik heb altijd enorm genoten van de dingen die we samen gedaan hebben. Maaike wij hebben elkaar bij de TSAC ontmoet en toen we er achter kwamen dat Black Slab voor ons beide de eerste klimroute in Cornwall was, kon een lange vriendschap niet uitblijven. Ik heb nooit getwijfeld om jou als paranimf te vragen. Merle, voor de vele etentjes en het nodige theeleuten. Hans, die eens een artikel van mij van onder tot boven heeft doorgespit om mijn engels op te vijzelen. Martin niet te vergeten voor de vrijwillige afdracht van de lay-out van zijn proefschrift. Tom, voor alle etentjes, klautertripjes en het mij door de laatste restjes van mijn promotie sleuren!

Natuurlijk kan ook mijn familie niet achterblijven in dit verhaal. Helaas heeft mijn vader het allemaal niet mee mogen maken. Mijn moeder en mijn zus Judith zijn de twee belangrijkste personen in mijn leven die altijd klaar staan waar dat nodig is. Die heb ik hard nodig gehad om dit verhaal tot een goed einde te kunnen brengen. Bedankt!

Eva

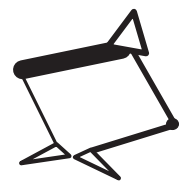


Dankwoord 


\section{List of publications}

\section{Journal articles}

- Wentink, E.C., Beijen, S.I., Hermens, H.J., Rietman, J.S., Veltink, P.H. "Intention detection of gait initiation using EMG and kinematic data in able-bodied subjects", Gait \& Posture 37(2):223-228;(2013.)

- Wentink, E.C., Stramigioli, S., Rietman, J.S., Koopman, H.F.J.M., Veltink, P.H. "Feasibility of energy efficient variable stiffness actuation to control a prosthetic knee - a modeling study", Medical Engineering and Physics, 35(6):838-845;(2013.)

- Wentink, E.C., Schut, V.G.H., Prinsen, E.C., Rietman, J.S., Veltink, P.H., "Intention detection of gait initiation using kinematic sensors and EMG in transfemoral amputees", accepted for publication in Gait \& Posture, (2013.)

- Wentink, E.C., Prinsen, E.C., Rietman, J.S., Veltink, P.H. "Changes in muscle activity patterns during slope and stair walking between transfemoral prosthetic users and controls", Journal of NeuroEngineering and Rehabilitation, 10(87):(2013.)

\section{Journal articles (in preparation)}

- Wentink, E.C., Prinsen, E.C., H.J., Rietman, J.S., Veltink, P.H. "Changes in muscle activity patterns during walking between transfemoral prosthetic users and controls"

- Wentink, E.C., E. Kerkdijk, Rietman, J.S., Veltink, P.H. "Feasibility of error-based electrotactile feedback in prosthetic walking", submitted as technical note

- Wentink, E.C., L. Alfring, Rietman, J.S., Veltink, P.H. "Feasibility of vibrotactile feedback inside the socket of upper leg prostheses" 


\section{Conference proceedings articles}

- Wentink, E.C., Mulder, A, Rietman, J.S., Veltink, P.H. "Vibrotactile stimulation of the upper leg: Effects of location, stimulation method and habituation", Proceedings 33rd Annual International Conference of the IEEE Engineering in Medicine and Biology Society, Boston, USA, p.1668-1671, (Aug. 2011)

- Wentink, E.C., Mulder, A, Rietman, J.S., Veltink, P.H. "Exploring the usability of pager motors for feedback in transfemoral amputees", Proceedings 3rd Dutch Biomedical Engineering Conference Egmond aan Zee, the Netherlands, p.137 (Jan. 2011)

- Wentink, E.C., Rietman, J.S., Veltink, P.H. "The feasibility of reflexive control in transfemoral prosthesis" Proceedings annual symposium of the IEEE EMBS Benelux, Enschede, the Netherlands, p.158 (Nov. 2009)

\section{Dutch articles}

- Wentink, E.C., Rietman, J.S., Veltink, P.H. "Reflex-leg, een energieefficiente en intelligente bovenbeenprothese", Orthopedische techniek, Sept (2011).

\section{Conference contributions (oral)}

- Wentink, E.C., Mulder, A, Rietman, J.S., Veltink, P.H. "Vibrotactile stimulation of the upper leg: Effects of location, stimulation method and habituation", 33rd Annual International Conference of the IEEE Engineering in Medicine and Biology Society, Boston, USA (Aug. 2011) 


\section{Propositions}

Accompanying the thesis

\section{"Feasibility of enhanced user control and feedback in upper leg prostheses"}

by Eva C. Wentink, to be publicly defended on the $25^{\text {th }}$ of October 2013

1. There are principle arguments to support that non-invasive feedback combined with user control will never be fast and accurate enough to improve the usability of upper leg prostheses. (Chapter 2)

2. Even though the leg is physically missing, it may still be present in the brain . (Chapter 4 and 5)

3. Detection of walking initiation does not require mind reading (Chapter 5 and 6)

4. A breakthrough in feedback for upper leg prostheses will be achieved when an artificial user feedback can match the speed and accuracy of human reflexive and/or visual system. (Chapter 7 )

5. The only good thing about wars and battles is the subsequent increased motivation for fast medical and technological developments.

6. Life is like rock climbing: it is good to have close friends, even if they are (a) little nuts.

7. Renovation of the residences in a neighborhood does not imply a renovation of the character of the neighborhood.

8. If your supervisor says it is not a lot of work, it usually is.

9. When women apply for a job, having a child may suddenly become "an accident waiting to happen".

10. Break a leg, avoid losing it! 